Modelo de efeito aleatório e erros de medida

Lourdes Coral Contreras Montenegro

TESE APRESENTADA AO

INSTITUTO DE MATEMÁTICA E ESTATÍSTICA

DA UNIVERSIDADE DE SÃO PAULO

PARA OBTENÇÃO DO TÍTULO DE DOUTOR EM CIÊNCIAS

Área de Concentração: Estatística

Orientador: Prof. Dr. Heleno Bolfarine

Durante a elaboração deste trabalho o autora recebeu apoio financeiro da CAPES

- São Paulo, março de 2006 - 


\section{Modelo de efeito aleatório e erros de medida}

Este exemplar corresponde à redação final da tese devidamente corrigida e defendida por Lourdes Coral Contreras Montenegro e aprovada pela comissão julgadora.

São Paulo, 13 de março de 2006.

Banca examinadora:

- Prof. Dr. Dr. Heleno Bolfarine (Orientador) - IME/USP

- Prof. Dr. Gilberto Alvarenga Paula - IME/USP

- Prof. Dr. Víctor Hugo Lachos Dávila - Bayes Forecast

- Prof. Dr. Enrico Antonio Colosimo - UFMG

- Prof. Dr. Mário de Castro Andrade Filho - ICMC/USP 
Ao meu marido Marcos

e aos meus pais 


\section{Agradecimentos}

- À meu orientador Prof. Dr. Heleno Bolfarine, por sua compreensão, dedicação e orientação, que de uma maneira clara e objetiva sempre me direcionou para um bom desenvolvimento desse trabalho.

- À meu co-orientador Prof. Dr. Victor Hugo Lachos Dávila, por aceitar ser meu co-orientador mesmo não sendo consolidado seu nome na pós-graduação, obrigado por sua dedicação, competência, companheirismo e por fazer-me sentir parte de sua família.

- Agradeço em especial a meu marido Marcos, pelo carinho, apoio e incentivo sabendo compreender os momentos de minha necessária ausência. A meus pais, por transformarme em uma pessoa responsável e dedicada à busca do conhecimento, e a meus irmãos e sobrinhos que sempre torceram por mim.

- Aos membros da banca examinadora, Prof. Mário de Castro e Prof. Victor Hugo Lachos, pelas sugestões e revisão ortográfica deste trabalho. Prof. Gilberto de Paula e Prof. Enrico Colosimo, pelas valiosos aportes e sugestões.

- A todos os professores e funcionários do IME-USP que, direta ou indiretamente, tenham colaborado na realização deste trabalho.

- Ao Cristian, pelas conversas e trocas de conhecimentos. 
- A todos os amigos(as) da pós-graduação da estatística, da sala de estudo do bloco $\mathrm{B}$, aos amigos(as) da comunidade peruana e colombiana do IME e finalmente aos amigos(as) da pensão, pelo prazer de suas amizades.

- À CAPES, pelo apoio financeiro.

- E finalmente a Deus, por te me dado saúde, disposição e por me oferecer inúmeras oportunidades nesta vida. 


\section{Resumo}

Uma suposição muito comum em modelos de regressão é assumir que as observações seguem uma distribuição normal. No entanto, esta suposição as vezes é irreal e pode ocultar importantes características do modelo. Neste trabalho apresentamos e estudamos os modelos de Grubbs e com intercepto nulo com erros de medida e também, modelos lineares mistos, considerando distribuições normais-assimétricas. Para validar tais modelos propomos técnicas de diagnóstico e as ilustramos com exemplos práticos. Para modelos com dados censurados e com erros de medida, conseguimos comparar procedimentos de estimação com e sem dados agrupados considerando distribuição log-normal e as ilustramos através de simulações. 


\section{Abstract}

A very common assumption in regression models is to assume that the observations follow a normal distribution. However, this assumption the times is unreal and can occult important characteristics of the model. In this work we present and study the models of Grubbs and with null intercept with errors of measurement and also linear mixed models, considering skew-normal distributions. To validate such models we consider diagnostic techniques and we illustrate them with practical examples. For models with censured data with errors of meadurement we obtain to compare procedures of estimation with and without grouped data considering the log-normal distribution and we illustrate them through simulation. 


\section{Índice}

$\begin{array}{lr}\text { Resumo } & 6\end{array}$

$\begin{array}{ll}\text { Abstract } & 7\end{array}$

Lista de Figuras $\quad$ vii

Lista de Tabelas $\quad$ xi

1 Introdução 1

1.1 Organização da tese . . . . . . . . . . . . . . 5

2 Tópicos de interesse $\quad 6$

2.1 Modelos de interesse . . . . . . . . . . . . . . 7

2.1 .1 Modelo de Grubbs . . . . . . . . . . . . . . 7

2.1.2 Modelo com intercepto nulo com erros de medida . . . . . . . 12 
2.1.3 Modelos lineares mistos . . . . . . . . . . . . . 13

2.1.4 Modelos de análise de sobrevivência . . . . . . . . . . . . 15

2.2 Distribuição normal-assimétrica . . . . . . . . . . . . . . . 20

2.2.1 Distribuição normal-assimétrica univariada de Azzalini . . . . 21

2.2.2 Distribuição normal-assimétrica de três parâmetros . . . . . . 23

2.2.3 Distribuição normal-assimétrica multivariada de Lachos . . . . 24

2.2.4 Distribuição normal-assimétrica univariada de Sahu . . . . . . 25

2.2.5 Distribuição normal-assimétrica multivariada de Sahu . . . . . 27

2.3 Algoritmo EM . . . . . . . . . . . . . . . . . . . . . 29

2.4 Critérios de informação . . . . . . . . . . . . . . . . 30

2.5 Influência local . . . . . . . . . . . . . . . . 31

2.5.1 Abordagem de Cox ... . . . . . . . . . . 31

2.5.2 Abordagem de Zhu e Lee. . . . . . . . . . . . . . . . . 34

\section{Modelo de Grubbs normal-assimé-}

$\begin{array}{ll}\text { trico } & 37\end{array}$

3.1 Introdução . . . . . . . . . . . . . . . . . . . 37

3.2 Especificação do modelo . . . . . . . . . . . . . . . . . 39

3.2 .1 Função Escore . . . . . . . . . . . . . . . . . . . . 40 40 
3.2.2 Matriz de informação de Fisher observada . . . . . . . . . . . 41

3.2.3 Estimação de máxima verossimilhança via algoritmo EM . . . 42

3.3 Esquemas de perturbação para o MGSN . . . . . . . . . . . . . . . . 45

3.3.1 Perturbação da ponderação de casos . . . . . . . . . . . . 45

3.3.2 Perturbação das medições de um dos instrumentos . . . . . . . 46

3.3.3 Perturbação do viés multiplicativo . . . . . . . . . . . . . 47

3.4 Aplicações . . . . . . . . . . . . . . . . . . . . . . . . . 48

3.4 .1 Dados de Grubbs . . . . . . . . . . . . . . . . . . . 48

3.4 .2 Dados de Barnett . . . . . . . . . . . . . . . . . . 51

4 Modelo com intercepto nulo com erros de medida normal-assimétrico

4.1 Introdução . . . . . . . . . . . . . . . . . . . . . . . . 59

4.2 Especificação do modelo para uma população . . . . . . . . . . . . 60

4.2 .1 Função Escore . . . . . . . . . . . . . . . . . . . . 62

4.2.2 Matriz de informação de Fisher observada . . . . . . . . . . . 63

4.2.3 Estimação de máxima verossimilhança via algoritmo EM . . . 64

4.3 Especificação do modelo para duas populações . . . . . . . . . . . . . 67

4.3.1 Estimação de máxima verossimilhança via algoritmo EM . . . 69 
4.3 .2 Influência local . . . . . . . . . . . . . . . 71

4.4 Esquemas de perturbação para o MINSN . . . . . . . . . . . . . 72

4.4.1 Perturbação da Ponderação de Casos . . . . . . . . . . . . . . 72

4.4 .2 Perturbação na variável resposta . . . . . . . . . . . . 73

4.4.3 Perturbação na variável explanatória . . . . . . . . . . . . . 74

4.5 Aplicação . . . . . . . . . . . . . . . . . . . . 75

5 Modelo linear misto normal-assimétrico

5.1 Introdução $: \ldots \ldots \ldots \ldots$. . . . . . . . . . . . . . 83

5.2 Especificação do modelo . . . . . . . . . . . . . . . . . . 84

5.2.1 Estimação de máxima verossimilhança via algoritmo EM . . . 86

5.3 Influência local . . . . . . . . . . . . . . . . 88

5.3 .1 Cálculo da matriz Hessiana $\ddot{Q}_{\boldsymbol{\theta}}(\widehat{\theta}) \ldots \ldots . \ldots 88$

5.3 .2 Esquemas de perturbação . . . . . . . . . . . . . . 9 90

5.4 Estudo de simulação . . . . . . . . . . . . . . . . . . 93

5.5 Aplicação . . . . . . . . . . . . . . . . . . . . 94

6 Modelo de tempo de falha acelerada com erro de medida 101 
6.1 Introdução . . . . . . . . . . . . . . . . . . . . . . . . . 101

6.2 Modelo sem efeito aleatório . . . . . . . . . . . . . . . 103

6.2.1 Estimação dos parâmetros . . . . . . . . . . . . . 106

6.2.2 Estimação da função de sobrevivência . . . . . . . . . . . 120

6.3 Modelo com efeito aleatório . . . . . . . . . . . . . . 125

6.3 .1 Estimação dos parâmetros . . . . . . . . . . . . . . 128

7 Considerações finais

7.1 Conclusões . . . . . . . . . . . . . . . . . . . . . . . . . . . 134

7.2 Pesquisas futuras $\ldots \ldots \ldots \ldots \ldots \ldots \ldots \ldots \ldots$

$\begin{array}{ll}\text { A Lemas Utilizados } & 137\end{array}$

B Demonstração do Teorema 3.1 139

C Cálculo das esperanças do passo E do algoritmo EM 141

D Cálculo da função escore do MGSN e MINSN 145

D.1 Função Escore do MGSN . . . . . . . . . . . . . . . . . . . . . 145

D.2 Função escore do MINSN . . . . . . . . . . . . . . . . . 147

E Obtenção da matriz de informação de Fisher observada $\quad 149$ 
E.1 Matriz de informação de Fisher observada do MGSN . . . . . . . . 149

E.2 Matriz de informação de Fisher observada do MINSN . . . . . . . . 152

F Obtenção da matriz delta

F.1 Matriz Delta para as Perturbações Aditiva e Multiplicativa do MGSN 156

F.2 Matriz delta para perturbação do viés multiplicativo do MGSN . . . . 157

F.3 Matriz delta para perturbação da variável resposta do MINSN . . . . 159

F.4 Matriz delta para perturbação da variável explanatória do MINSN . . 160

G Programas

G.1 Programa em MATLAB que calcula os EMV do MGSN usando o algoritmo EM . . . . . . . . . . . . . . . . . . 162

G.2 Programa em MATLAB do gráfico PP tipo Healy segundo Azzalini e Capitanio do MGSN . . . . . . . . . . . . . . . 165 


\section{Lista de Figuras}

2.1 Função de densidade da normal-assimétrica para $\lambda=-5,-3,0,3,5 . \quad$. 22

2.2 Contornos da distribuição normal-assimétrica bivariada para diferentes valores de $\lambda$ : (a) $\lambda_{1}=0$ e $\lambda_{2}=0$, (b) $\lambda_{1}=-0.7$ e $\lambda_{2}=0.9$, (c) $\lambda_{1}=0.9$ e $\lambda_{2}=-0.7$ e (d) $\lambda_{1}=0.2$ e $\lambda_{2}=0.9 \ldots \ldots \ldots \ldots . \ldots \ldots$

3.1 Dados de Grubbs. Gráfico PP tipo Healy para (a) modelo Grubbs normal-assimétrico e (b) modelo Grubbs normal. . . . . . . . . . . . . 55

3.2 Dados de Grubbs, interesse em $\boldsymbol{\theta}$, perturbação de casos. Gráfico de índice de (a) $|d \max |$, (b) $C_{i}$, (c) afastamento da verossimilhança $L D_{i}$ e (d) distância de Cook $D_{i}$. . . . . . . . . . . . . . . . 56

3.3 Dados de Grubbs, interesse em $\boldsymbol{\theta}$. Gráfico de índice de $|d m a x|$ para (a) Perturbação aditiva e (b) perturbação multiplicativa das medidas obtidas pelo observador A. . . . . . . . . . . . . .

3.4 Diagnóstico dos dados de Grubbs para MGN, interesse em $\boldsymbol{\theta}$. Gráfico de índice de (a) $C_{i}$ para a perturbação de ponderação de casos e (b) distância de $\operatorname{Cook} D_{i} \ldots \ldots \ldots \ldots \ldots \ldots$ 
3.5 Dados de Barnett. Gráficos PP tipo Healy para (a) modelo Grubbs normal-assimétrico e (b) modelo Grubbs normal. . . . . . . . . . . . . 59

3.6 Dados de Barnett. Gráfico de índice de (a) $\left|d_{\max }\right|$ e (b) $C_{i}$ para a perturbação de viés multiplicativo. . . . . . . . . . . . 5 5

4.1 Conjunto de dados de índice de placa dentária. (a) Gráfico PP tipo Healy do MINSN (linha contínua) e MINN (linha tracejada). (b) Histograma da covariável observada X (índice de placa no início do estudo) sobreposto pelas densidades estimadas das distribuições normalassimétrica (linha contínua) e normal (linha tracejada) . . . . . . . .

4.2 Conjunto de dados de índice de placa dentária, interesse em $\boldsymbol{\theta}$. Gráfico de índice: (a) $|d \max |$ e (b) $C_{j}$ para a perturbação da ponderação de casos. (-) e (...) denotando o gráfico de índice para MINSN e MINN, respectivamente. ..................... 80

4.3 Conjunto de dados de índice de placa dentária, modelo MINSN. Gráfico de índice: (a) Afastamento da verossimilhança, $L D_{j}$ e (b) distância de Cook, $D_{j} \ldots \ldots \ldots \ldots \ldots \ldots \ldots \ldots \ldots \ldots \ldots \ldots \ldots \ldots \ldots \ldots$

4.4 Conjunto de dados de índice de placa dentária, interesse em $\boldsymbol{\theta}$. Gráfico de índice: (a) $|d \max |$ para perturbação da variável resposta e (b) $|d \max |$ para perturbação da variável explanatória. (-) e (...) denotando o gráfico de índice para os MINSN e MINN, respectivamente. 
5.1 Conjunto de dados simulados. Gráfico de índice (a) $C_{i}$ para perturbação de ponderação de casos, (b) $|d \max |$ para perturbação da matriz de escala da efeitos aleatórios, (c) $C_{i}$ para perturbação da variável resposta e (d) afastamento da verossimilhança $L D_{i}^{c} \ldots \ldots \ldots \ldots$

5.2 Conjunto de dados de Colesterol de Framingham. (a) Histograma dos níveis de colesterol sobreposta pela densidade estimada kernel e (b) gráfico de contornos da densidade estimada de $\mathrm{b}_{i} \ldots \ldots \ldots \ldots$

5.3 Conjunto de dados de Colesterol de Framingham, interesse em $\boldsymbol{\theta}$. Gráfico de índice: (a) $|d \max |$ e (b) $\left|C_{i}\right|$ para perturbação da ponderação de casos . . . . . . . . . . . . . . . . . . . . . . . . . . . 98

5.4 Conjunto de dados de Colesterol de Framingham, interesse em $\boldsymbol{\theta}$. Gráfico de índice: (a) $|d \max |$, (b) $\left|C_{i}\right|$ para perturbação da matriz de escala dos efeitos aleatórios, (c) $|d \max |$ para perturbação das variáveis explanatórias e (d) $\left|C_{i}\right|$ para perturbação das variáveis respostas. . . .

5.5 Conjunto de dados de Colesterol de Framingham. Gráfico de medidas da (a) Afastamento da verossimilhança $L D_{i}^{c}$ e (b) distância de Cook $D_{i}^{c} .100$

6.1 Curvas de sobrevivência teórica e ajustadas, com base nas médias das estimativas dos parâmetros, tamanho de amostra 50 e $0 \%$ de censura, com erro de medida. Resultados para diferentes valores da variância do erro de medida $\left(\sigma_{\xi}^{2}\right)$. (a) Método de máxima verossimilhança, (b) Método SIMEX, (c) Método regressão-calibração e (d) Método ingênuo.123 
6.2 Curvas de sobrevivência teórica e ajustada, com base nas médias das estimativas dos parâmetros, tamanho de amostra 50 e $30 \%$ de censura, com erro de medida. Resultados para diferentes valores da variância do erro de medida $\left(\sigma_{\xi}^{2}\right)$. (a) Método de máxima verossimilhança, (b) Método SIMEX, (c) Método regressão-calibração e (d) Método ingênuo.124 


\section{Lista de Tabelas}

3.1 Conjunto de dados de Grubbs. Tempo de queima de projéteis em segundos, medido por cada um dos três observadores. . . . . . . . . . 51

3.2 Resultados do ajuste do modelo de Grubbs normal e normal-assimétrico (MGN e MGSN) para o conjunto de dados de Grubbs. EP são as estimativas dos erros padrão assintóticos baseados na matriz de informação de Fisher observada apresentada no Apêndice E.1. . . . . . .

3.3 Resultados do ajuste do modelo de Grubbs normal-assimétrico (MGN e MGSN) para os dados de Barnett (1969). EP são estimativas dos erros padrão assintóticos baseados na matriz de informação de Fisher observada dada no Apêndice E.1.

4.1 Resultado do ajuste do modelo de intercepto nulo com erro de medida normal-assimétrico e normal (MINSN e MINN) para o conjunto de dados de índice de placa dentária. EP são erros padrão estimados assintóticamente baseados na matriz de informação. . . . . . . . . . 78

4.2 Conjunto de dados referente a enxágüe bucal. . . . . . . . . . . . . 81 
5.1 Resultados do ajuste do modelo misto normal-assimétrico e normal (MLMSN e MLMN) para o conjunto de dados de colesterol de Framingham. $d_{11}, d_{12}$ e $d_{22}$ são os distintos elementos da matriz de covariâncias $\mathbf{D}^{1 / 2}$. EP são erros padrão estimados assintóticamente baseados na matriz hessiana. . . . . . . . . . . . . . . . .

6.1 Média das estimativas de Monte Carlo dos parâmetros e correspondente erro padrão, com variância do erro de medida $\sigma_{\xi}^{2}=0,3 \mathrm{e}$ tamanho de amostra $n=50$. Verdadeiros valores: $\alpha=0,5 ; \beta=1,0$; $\mu_{x}=1,0 ; \sigma_{x}^{2}=1,0$ e $\sigma_{\epsilon}^{2}=0$,

6.2 Média das estimativas de Monte Carlo dos parâmetros e correspondente erro padrão, com variância do erro de medida $\sigma_{\xi}^{2}=0,3 \mathrm{e}$ tamanho de amostra $n=250$. Verdadeiros valores: $\alpha=0,5 ; \beta=1,0$; $\mu_{x}=1,0 ; \sigma_{x}^{2}=1,0$ e $\sigma_{\epsilon}^{2}=0,5 \ldots \ldots \ldots$

6.3 Média das estimativas de Monte Carlo dos parâmetros e correspondente erro padrão, com variância do erro de medida $\sigma_{\xi}^{2}=0,5 \mathrm{e}$ tamanho de amostra $n=50$. Verdadeiros valores: $\alpha=0,5 ; \beta=1,0$; $\mu_{x}=1,0 ; \sigma_{x}^{2}=1,0$ e $\sigma_{\epsilon}^{2}=0,5 \ldots \ldots \ldots \ldots \ldots \ldots \ldots$

6.4 Média das estimativas de Monte Carlo dos parâmetros e correspondente erro padrão com variância do erro de medida $\sigma_{\xi}^{2}=0,5$ e tamanho de amostra $n=250$. Verdadeiros valores: $\alpha=0,5 ; \beta=1,0 ; \mu_{x}=1,0$;

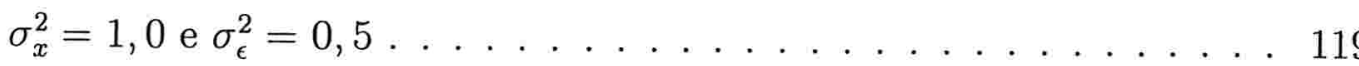


6.5 Média das estimativas de Monte Carlo, média da distribuição a posteriori dos parâmetros e os correspondentes erros padrão, com variância do erro de medida $\sigma_{\xi}^{2}=0,3$ e tamanho de amostra $n=50$ para cada grupo $j=1,2$ e 3 . Verdadeiros valores: $\alpha=0,5 ; \beta=1,0 ; \mu_{x}=1,0$;

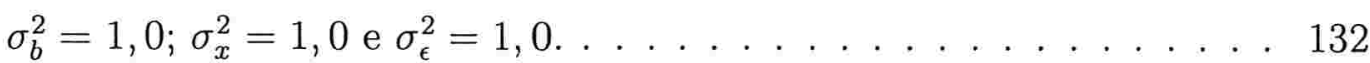

6.6 Média das estimativas de Monte Carlo, média da distribuição a posteriori dos parâmetros e os correspondentes erros padrâo com variância do erro de medida $\sigma_{\xi}^{2}=0,5$ e tamanho de amostra $n=50$ para cada grupo $j=1,2$ e 3 . Verdadeiros valores: $\alpha=0,5 ; \beta=1,0 ; \mu_{x}=1,0$; $\sigma_{b}^{2}=1,0 ; \sigma_{x}^{2}=1,0$ e $\sigma_{\epsilon}^{2}=1,0$. 


\section{Capítulo 1}

\section{Introdução}

Assumir que as observações seguem uma distribuição normal é uma suposição muito comum em modelos de regressão. No entanto, esta suposição pode ser irreal e pode ocultar importantes características de variação que se encontra presente nos dados. Este trabalho concentra-se em estudar os seguintes modelos: modelo de Grubbs (1948, 1973), originalmente desenvolvido para comparar instrumentos que medem a característica de interesse na mesma escala, por exemplo na área industrial. Sabemos que, métodos de medida freqüentemente diferem em custo, velocidade ou outros fatores. Estes vários instrumentos podem ser um instrumento avaliado várias vezes e nosso interesse seria se o vício e a precisão dos instrumentos variam com o tempo. Freqüentemente a avaliação dos instrumentos é feita baseada em dados obtidos utilizando cada instrumento para medir uma característica comum em várias unidades experimentais. Em muitas situações, a característica populacional a ser mensurada, como por exemplo concentração de ouro em amostras de solo (Galea-Rojas et al., 2003), a capacidade pulmonar de pacientes (Barnett, 1969), entre outros, parecem seguir um comportamento assimétrico. 
Estudamos também o caso do modelo de regressão passando pela origem, ou seja, com intercepto nulo. A motivação para considerar tal modelo está ligada ao conjunto de dados estudados por Hadgu e Koch (1999), que visa comparar dois tipos de enxágüe bucal (A e B) ou controle (placebo). Uma avaliação da eficácia dos dois tipos de enxágüe na remoção de placa dentária antes $(x)$ (no início do estudo ou "baseline") e depois (y) (três e seis meses) do uso do enxágüe bucal. A covariável índice de placa dentária antes $(x)$ do uso de um enxagüe bucal parece apresentar um comportamento assimétrico. Os autores analisaram este conjunto de dados usando equações de estimação generalizada, assumindo distribuição gama para a variável resposta.

Observe que as covariáveis dos modelos de Grubbs e com intercepto nulo estão sujeitos a erros de medição, uma forma alternativa para analisar os conjuntos de dados de cada um deles é considerando modelos com erros de medida.

No caso do modelo linear misto multivariado, a motivação está ligada ao fato de considerar o conhecido estudo de colesterol de Framingham (Zhang e Davidian, 2001), onde uma característica dos dados investigados, como níveis de colesterol, parece seguir uma distribuição assimétrica.

Sabemos que do ponto de vista prático, o método mais adotado para alcançar a normalidade é a transformação de variáveis que funciona bem em muitos casos. Embora esta metodologia possa fornecer resultados empíricos razoáveis, esta deve ser evitada se um modelo teórico mais conveniente for encontrado (Azzalini e Capitanio, 1999). Assim, considerável esforço tem sido direcionado para relaxar a suposição de normalidade. Uma alternativa interessante é ajustar os modelos mencionados acima baseados em famílias paramétricas de distribuições que mostrem um 
bom comportamento, similar à distribuição normal, e que possam estar arbitrariamente próxima à distribuição normal dependendo de parâmetros convenientes. Neste sentido, a família normal assimétrica de distribuições que é analiticamente tratável, acomoda valores práticos de asimétria e curtose estritamente inclui a distribuição normal como um caso especial. Assim, muitos autores tais como Azzalini (1985), Azzalini e Capitanio (1999), Azzalini e Kotz (2002), Capitanio et al. (2002), Sahu e Dey (2000), Sahu et al. (2003) e Arellano-Valle et al. (2005), entre outros, estudaram modelos de regressão com distribuições assimétricas, mas não existe literatura no contexto de modelos de Grubbs e modelos com intercepto nulo. Para modelos lineares mistos Lachos (2004), estuda este modelo assumindo que tanto os erros como os efeitos aleatórios seguem uma distribuição normal-assimétrica. Neste trabalho, assumimos que os efeitos aleatórios do modelo seguem uma distribuição normal-assimétrica, sendo esta distribuição uma extensão univariada proposta por Sahu et al. (2003). A vantagem desta distribuição em relação a Lachos (2004), é trabalhar a parte teórica do modelo diretamente com o parâmetro de assimétria sem considerar reparametrizações complexas, e na parte computacional, conseguimos algoritmos eficientes para obter estimadores de máxima verossimilhança.

Para os modelos mencionados acima, obtemos a distribuição marginal das observações e implementamos a metodologia de máxima verossimilhança utilizando o algoritmo EM (Dempster et al., 1977). Implementamos também a metodologia de influência local para investigar a influência de pequenas perturbações do modelo no estimador de máxima verossimilhança.

Para modelos com dados censurados, trabalhamos com o modelo de locação e escala, também conhecido como modelo de vida acelerado (Cox e Oakes, 1984) ou, com mais frequêntemente, modelo de tempo de falha acelerado (Keinding et al., 1997, 
entre outros). Existem diversos estudos na literatura que tratam de problema de covariáveis com erro de medida, sendo quase todos com base na verossimilhança parcial usada no contexto de Cox (1975). Em relação aos modelos de tempo de falha acelerado com erro de medida, temos alguns resultados para o modelo de regressão Weibull. Gimenez et al. (1999) apresentam um estudo de simulação para comparar propriedades de diferentes estimadores do coeficiente angular do modelo com diferentes níveis de censura. Estudos referentes a modelos de tempo de falha acelerado com efeito aleatório, para o caso univariado são encontrados, por exemplo, nos trabalhos de Pettitt (1986), Lawless (1982) e Hougaard (1987), enquanto no caso multivariado temos o trabalho de Klein et al. (1999), entre outros. No entanto, existe uma escassa literatura neste último contexto tratando de modelos com erros de medida.

Neste trabalho pretendemos fazer um estudo de simulação para comparar propriedades de diferentes estimadores. Para o modelo de tempo de falha acelerado com erro de medida consideramos a distribuição log-normal na variável resposta. As distribuições marginais serão obtidas com o objetivo de usar vários métodos de estimação como máxima verossimilhança, simulação e estrapolação (SIMEX), proposto por Cook e Stefanski (1994), regressão-calibração estudado em Carroll et al. (1995) e discutimos um procedimento de maximização alternativa da verossimilhança baseado no algoritmo EM. Para modelos de tempo de falha acelerada com efeito aleatório com erro de medida, usamos o método de máxima verossimilhança para a estimação dos parâmetros deste modelo. Adicionalmente, pretendemos também comparar os resultados de estimação derivadas através do enfoque bayesiano com os resultados clássico, com o intuito de comparar os estimadores do modelo. 


\subsection{Organização da tese}

A presente tese de doutorado está dividida em sete capítulos e sete apêndices. No Capítulo 2 apresentamos uma revisão bibliográfica dos modelos em estudo, das distribuições normais-assimétricas, dos critérios de informação, o algoritmo EM e as técnicas de avaliação de influência local. No Capítulo 3 apresentamos um estudo sobre o modelo de Grubbs normal-assimétrico, influência local e analisamos dois conjuntos de dados reais utilizando os resultados obtidos neste capítulo. Modelo com intercepto nulo com erro de medida considerando a distribuição normal-assimétrica para uma população, depois estendemos os resultados para duas populações, influência local e uma aplicacção a um conjunto de dados reais são estudados no Capítulo 4. No Capítulo 5 apresentamos o modelo linear misto considerando a distribuição normalassimétrica para os efeitos aleatórios, estudo de influência local são mostrados e como aplicação analisamos um conjunto de dados reais. No Capítulo 6 apresentamos o modelo de tempo de falha acelerada com distribuição log-normal juntamente com erro de medida. Ainda neste capítulo abordamos o modelo de tempo de falha acelerado com efeito aleatório e distribuição log-normal considerando erros de medida. Neste caso, fazemos comparações entre as abordagens clássica e bayesiana. Finalmente, no Capítulo 7 apresentamos as conclusões, incluindo sugestões para trabalhos futuros a serem desenvolvidos. 


\section{Capítulo 2}

\section{Tópicos de interesse}

Neste capítulo apresentamos uma breve introdução dos modelos estudados neste trabalho, revisamos as metodologias da distribuição normal-assimétrica para os casos univariados e multivariados considerando as definições proposta por Azzalini (1985) e Sahu et al. (2003). Para a estimação dos parâmetros apresentamos o algoritmo EM utilizados nos modelos em estudo. Para seleção dos modelos ao compararmos modelos normal e norma-assimétrico descrevemos três critérios de informação utilizados na literatura. Finalmente, dentre as abordagens mais utilizadas na prática, para medir a influência em modelos de regressão linear, destacam-se as análises baseadas em inlfuência local através de duas abordagens, uma dás mais conhecidas na literatura é a de Cook (1986) e uma outra abordagem considerando dados incompletos temos a de Zhu e Lee (2001). 


\subsection{Modelos de interesse}

Nesta seção fazemos uma revisamos dos modelos utilizados neste trabalho, consi deramos a suposição de normalidade comumente encontrada na literatura em características específicas de cada modelo a ser estudado, tais como, modelo de Grubbs $ə$ e modelo com intercepto nulo com erros de medida, modelos lineares mistos e por fim o modelo de tempo de falha acelerado com erros de medida com e sem efeito aleatório.

\subsubsection{Modelo de Grubbs}

Calibração estatística é importante em aplicações práticas de diversas áreas, tais como na Engenharia, na aferição de instrumentos de Medição de grandezas físicas; na medicina é importante para a calibração de instrumentos indicadores do estado de saúde do paciente, e também são relatadas aplicações na Biologia e na Química. Neste último caso, o interesse é voltado para determinar concentrações de soluções e composições de materiais, por exemplo, a calibração estatística é o processo através do qual a escala de um instrumento de medição é determinada ou ajustada a partir de um instrumento de calibração ou de referência. Na calibração estatística é importante fazer distinção entre calibração absoluta, que é uma técnica para a obtenção de medidas de forma rápida ou não-padrão, calibradas por uma medida conhecida ou com erro experimental desprezível e na calibração comparativa, onde a idéia é determinar a relação entre diversos testes ou instrumentos que fornecem medidas indiretas similares. Nestes últimos anos tem se mostrado relevante a comparação de instrumentos de medição como, por exemplo, podemos citar um dos primeiros estudos nesta área, apresentado por Grubbs $(1948,1973)$, relatando comparação de três cronômetros. Barnett (1969) apresenta um exemplo onde quatro combinações 
instrumento-operador conhecidos para medir a capacidade vital num grupo de pacientes são avaliados. Leurgans (1980) compara dois métodos para medir a concentração da glicose no sangue e Jaech (1985) apresenta vários exemplos na área industrial. Christensen e Blackwood (1993), comparam cinco termopares, GaleaRojas (1995) apresenta um estudo de inferência no modelo t de Student e Bedrick (2001) compara três métodos para medir sedimentos encontrados no solo. Grubbs $(1948,1973)$ apresenta uma classe particular de modelos utilizados para comparar instrumentos de medição. O modelo conhecido na literatura como modelo de Grubbs supondo $(p>2)$ instrumentos é definido pela seguinte relação

$$
y_{i j}=\alpha_{j}+x_{i}+\epsilon_{i j}, \quad i=1, \ldots, n, j=1, \ldots, p,
$$

em que

$y_{i j}$ é a medida fornecida pelo instrumento $j$ para a característica da unidade $i$,

$\alpha_{j}$ é o vício aditivo do instrumento $j$,

$x_{i}$ é o verdadeiro valor da característica de interesse na unidade $i$,

$\epsilon_{i j}$ é o erro aleatório de medição, sendo $\epsilon_{i j}$ não correlacionados $i=1, \ldots, n, j=$ $1, \ldots, p$

$$
E\left(\epsilon_{i j}\right)=0, \quad \operatorname{Var}\left(\epsilon_{i j}\right)=\phi_{j} .
$$

O modelo definido em (2.1)-(2.2) é conhecido como uma classe particular de modelos de regressão linear com erros de medida (Fuller, 1987). Sabemos que na prática existem várias situações onde a variável explanatória não pode ser observada diretamente, mas com erros. Entre outras situações, a inexatidão da medida pode ser resultado de uma opinião subjetiva, ou do uso de instrumentos de precisão limitada. De acordo com Morgan e Elashoff (1987), uma importante fonte de má classificação em estudos da área médica é a dificuldade dos pacientes em relembrar eventos do passado, como história familiar de uma doença ou hospitalizações anteriores. Em estudos nutri- 
cionais, covariáveis relacionadas ao consumo de gordura saturada envolvem erros de medida consideráveis, em parte devido a uma tendência de algumas pessoas em omitir a descrição precisa de suas refeições (Carroll et al., 1995). Outra situação ocorre com o interesse em relacionar a produção de certo cereal com o nível de nitrogêneo disponível no solo (Fuller, 1987). Para termos o conhecimento do nível de nitrogêneo disponível no solo, é necessário amostrarmos o solo da unidade experimental e fazer análises laboratoriais na amostra selecionada. Como resultado da amostragem e da análise laboratorial, não temos os verdadeiros valores da covariável mas sim estimativas destas. Na estrutura sobre modelos com erros de medida, consideram-se três formas de modelagem das quantidades $x_{i}$ :

(i) As $x_{i}^{\prime} s$ são constantes desconhecidas, chamadas de parâmetros incidentais.

(ii) As $x_{i}^{\prime} s$ são variáveis aleatórias não-correlacionadas com média $\mu_{x}$ e variância $\phi_{x}$ e não são correlacionadas com os $\epsilon_{i j}$.

(iii) As $x_{i}^{\prime} s$ são variáveis aleatórias não-correlacionadas com média $\mu_{x i}$ e variância $\phi_{x}$ e são não correlacionadas com os $\epsilon_{i j}$.

O modelo descrito por (i) e (2.1)-(2.2) é denominado modelo funcional de Grubbs, neste caso o número de parâmetros cresce com o número de observações. O modelo descrito em (ii) e (2.1)-(2.2) é denominado modelo estrutural de Grubbs, neste modelo não é possível encontrar uma solução de máxima verossimilhança para os parâmetros do modelo, pois o mesmo não é identificável, no sentido que dois vetores de parâmetros diferentes podem dar origem a uma mesma distribuição. Finalmente, o modelo descrito por (iii) e (2.1)-(2.2) é denominado modelo ultraestrutural. Neste caso, os modelos funcional e estrutural podem ser vistos como casos especiais deste modelo. 
Do ponto de vista de aplicação, o modelo funcional é adequado quando temos interesse apenas nos indivíduos participantes do estudo, enquanto que o estrutural é adequado quando o interesse é generalizar os resultados do estudo para a população de onde vem os indivíduos envolvidos no estudo.

Neste trabalho, consideramos o modelo estrutural definido em (ii) e (2.1)-(2.2) que pode ser escrito em forma matricial como:

$$
\mathrm{Y}_{i}=\mathbf{a}+\mathbf{1}_{p} x_{i}+\epsilon_{i}, i=1, \ldots, n
$$

em que $\mathbf{a}=\left(\alpha_{1}, \ldots, \alpha_{p}\right)^{\top}, \mathbf{Y}_{i}=\left(Y_{i 1}, \ldots, Y_{i p}\right)^{\top}$ e $\epsilon_{i}=\left(\epsilon_{i 1}, \ldots, \epsilon_{i p}\right)^{\top}$ são vetores aleatórios de dimensão $p \times 1$ e $1_{p}$ é um vetor $p \times 1$ com todos os elementos iguais à unidade. Note que

$$
E\left(\mathbf{Y}_{i}\right)=\mathbf{a}+\mathbf{1}_{p} \mu_{x} \text { e } \operatorname{Var}\left(\mathbf{Y}_{i}\right)=D(\phi)+\phi_{x} \mathbf{1}_{p} \mathbf{1}_{p}^{\top}=\mathbf{\Sigma}
$$

em que $D(\phi)=\operatorname{diag}\left(\phi_{1}, \ldots, \phi_{p}\right)$ é uma matriz diagonal $p \times p, \phi=\left(\phi_{1}, \ldots, \phi_{p}\right)^{\top}$ com $\boldsymbol{\theta}=\left(\mu_{x}, \mathbf{a}^{\top}, \phi_{x}, \phi^{\top}\right)^{\top}$. Sob as condições acima o modelo estrutural (2.3) não é identificável, isto é, existem $\boldsymbol{\theta}_{1}$ e $\boldsymbol{\theta}_{2}$ diferentes tais que $F\left(\mathbf{y}, \boldsymbol{\theta}_{1}\right)=F\left(\mathbf{y}, \boldsymbol{\theta}_{2}\right)$, para todo $\mathbf{Y} \in \mathbf{R}$, onde $F$ é a função de distribuição de $\mathbf{Y}_{i}, i=1, \ldots, n$. Por exemplo, para o caso de $p=3$, sejam $\boldsymbol{\theta}_{1}=(1,1,1,1,1,1,1,1)^{\top}$ e $\boldsymbol{\theta}_{2}=(4,-2,-2,-2,1,1,1,1)^{\top}$. De (2.3) é fácil verificar que $\boldsymbol{\theta}_{1}$ e $\boldsymbol{\theta}_{2}$ geram a mesma média e a mesma matriz de covariâncias, isto é,

$$
E\left(\mathbf{Y}_{i}\right)=(2,2,2)^{\top} \text { e } \operatorname{Var}\left(\mathbf{Y}_{i}\right)=\left[\begin{array}{lll}
2 & 1 & 1 \\
1 & 2 & 1 \\
1 & 1 & 2
\end{array}\right]
$$

Daí a necessidade de reduzirmos o número de parâmetros desconhecidos, impondo alguma suposição adicional. Neste caso será necessário o conhecimento adicional de 
algum parâmetro do modelo para possibilitar a estimação dos parâmetros de maior interesse. Neste caso, o vetor de parâmetros do modelo é dado por

$$
\boldsymbol{\theta}=\left(\mu_{x}, \alpha_{1}, \ldots, \alpha_{p}, \phi_{x}, \phi_{1}, \ldots, \phi_{p}\right)^{\top}
$$

A falta de identificabilidade implica ausência de estimadores consistentes para todos os parâmetros, o que tem sérias implicações na teoria assintótica de estimação e testes de hipóteses. No entanto, o fato de ser identificável não implica que exista um estimador consistente para $\boldsymbol{\theta}$ (ver Gabrielsen, 1978). Uma forma de contornar o problema de falta de identificabilidade é restringir o parâmetro $\boldsymbol{\theta}$. Da mesma forma que Barnett (1969), assumimos que existe um método de medição, denominado método de referência ou de controle, que mede $x$ sem vício. Supomos que o instrumento de referência seja o número um. Assim, temos que $\alpha_{1}=0$. Logo, o modelo proposto por Grubbs $(1948,1973)$ considerando um instrumento de referência pode ser escrito da seguinte forma:

$$
\mathrm{Y}_{i}=\mathbf{a}+1_{p} x_{i}+\epsilon_{i}, i=1, \ldots, n
$$

em que $\mathbf{a}=\left(0, \boldsymbol{\alpha}^{\top}\right)^{\top}=\left(0, \alpha_{2}, \ldots, \alpha_{p}\right)^{\top} \operatorname{com} \boldsymbol{\alpha}=\left(\alpha_{2}, \ldots, \alpha_{p}\right)^{\top}, \mathbf{Y}_{i}$ e $\boldsymbol{\epsilon}_{i}$ como em (2.3). Agora, sob a condição de identificabilidade, o vetor de parâmetros em (2.6) se reduz a um vetor de dimensão $(2 p+1) \times 1$ dado por

$$
\boldsymbol{\theta}=\left(\mu_{x}, \boldsymbol{\alpha}^{\top}, \phi_{x}, \phi^{\top}\right)^{\top}
$$

$\mathrm{Na}$ literatura encontram-se vários trabalhos utilizando o modelo de Grubbs onde a análise é feita desde um ponto de vista multivariado, onde não é necessário preocuparse com a falta de identificabilidade. Veja, por exemplo, Christensen e Blackwood (1993) e Brindley e Bradley (1995), entre outros. Suponha que as observações de características de interesse $x$ seguem representadas por uma distribuição normal. Tomando novamente o modelo definido em (2.7), o modelo normal é obtido ao con- 
siderar

$$
\epsilon_{i} \stackrel{i i d}{\sim} N_{p}(0, D(\phi)) \text { e } x_{i} \stackrel{i i d}{\sim} N_{1}\left(\mu_{x}, \phi_{x}\right)
$$

com $\epsilon_{i}$ e $x_{i}$ independentes, $i=1, \ldots, n$. De (2.7), (2.8) e das propriedades da distribuição normal temos que

$$
\mathrm{Y}_{i} \stackrel{i i d}{\sim} N_{p}(\boldsymbol{\mu}, \boldsymbol{\Sigma}), \quad i=1, \ldots, n
$$

em que $\boldsymbol{\mu}=\mathbf{a}+\mathbf{1}_{p} \mu_{x}$ e $\boldsymbol{\Sigma}=\phi_{x} \mathbf{1}_{p} \mathbf{1}_{p}^{\top}+D(\phi), \operatorname{com} D(\phi)=\operatorname{diag}\left(\phi_{1}, \ldots, \phi_{p}\right)^{\top}$ e $\phi=\left(\phi_{1}, \ldots, \phi_{p}\right)$.

\subsubsection{Modelo de regressão com erro de medida e intercepto nulo}

$\mathrm{Na}$ literatura, a motivação do modelo de regressão com erro de medida origina-se do conjunto de dados da área odontológica apresentado em Singer e Andrade (1997). O estudo visa comparar dois tipos de escova avaliando a eficácia na remoção de placa dentária antes (pré-tratamento) e depois (pós-tratamento) da escovação. Como destacado em Singer e Andrade (1997), índice de placa pré-tratamento nula, acarreta índice de placa pós-tratamento nula, levando assim, a um modelo com intercepto nulo. Além disso, como as medidas de índice de placa dentária tomadas em cada criança não são precisas, Aoki et al. (2001) consideram o modelo com erro de medida com intercepto nulo para populações dependentes. Para este modelo, os estimadores de máxima verossimilhança são obtidos considerando a distribuição normal e a distribuição assintótica dos estimadores são obtidos em Aoki et al. (2002) considerando os estimadores pelo método dos momentos para a mesma classe de modelos. Recentemente, Aoki et al. (2003), do ponto de vista bayesiano discutem o modelo de regressão com erro de medida e intercepto nulo normal multivariado com estrutura 
de dependência entre as variáveis respostas dentro do mesmo grupo. Os autores aplicaram o modelo a um conjunto de dados reais apresentado em Hadgu e Koch (1999), o estudo objetiva comparar dois tipos de enxágue bucal A, B ou controle avaliando a eficácia na remoção de placa dentária antes (no início do estudo ou "baseline") e depois (três e seis meses) do uso de enxágüe bucal mencionado acima. As características de imprecisão em medir o índice de placa dentária e de considerar um modelo com intercepto nulo destacado em Aoki et al. (2001) são similares para este conjunto de dados.

O modelo de regressão com erro de medida e intercepto nulo considerado neste trabalho, é expresso por

$$
\begin{aligned}
& X_{k i j}=x_{k i j}+u_{k i j}, \\
& y_{k i j}=\beta_{k i} x_{k i j}+\epsilon_{k i j},
\end{aligned}
$$

em que $X_{k i j}$ e $y_{k i j}$ denotam, respectivamente, a variável explanatória e os valores observados das variáveis resposta para a população $k$, grupo $i$ e o indivíduo $j$, $\left(i=1, \ldots, p, j=1, \ldots, n_{i}, k=1, \ldots, r\right)$. Para este modelo consideramos o modelo estrutural, isto é, $x_{k i j}$, é uma variável aleatória correspondente aos valores verdadeiros de $X_{k i j}$ (índice de placa observada no início do tratamento), $\beta_{k i}, i=1, \ldots, p, k=$ $1, \ldots, r$, são os parâmetros de inclinação (desconhecidos). Usualmente é assumido que $x_{k i j}, \epsilon_{k i j}$ e $u_{k i j}$ são mutuamentes independentes, com distribuições $N\left(\mu_{x}, \phi_{x}\right)$, $N\left(0, \sigma_{i}^{2}\right), i=1, \ldots, p$, e $N\left(0, \sigma_{u}^{2}\right)$, respectivamente.

\subsubsection{Modelos Lineares Mistos}

O modelo linear misto (MLM) tem sido freqüentemente usado na análise de medidas repetidas, dados agrupados e dados longitudinais, entre outros. Este modelo tem 
uma grande aplicabilidade em diversas áreas de pequisa como agricultura, biologia, economia, geofísica e ciências sociais (Diggle et al., 1994). A importância destes modelos é explicada pela flexibilidade que eles oferecem para modelar a correlação entre e intra-unidades amostrais, freqüentemente presente em dados longitudinais (Laird e Ware, 1982) e dados agrupados (Henderson, 1984), pela capacidade de modelar dados balanceados e desbalanceados e por oferecer programas computacionais eficientes e confiáveis para ajustá-los (Wolfinger et al., 1991). Os modelos lineares mistos têm como casos particulares o modelo linear clássico, o modelo de componentes de variância e também os modelos hierárquicos (multiníveis) (Natis, 2002).

Em geral, supõe-se que tanto os erros como os efeitos aleatórios são normalmente distribuídos. Sob estas suposições, inferências para os parâmetros fixos e efeitos aleatórios do modelo podem ser tratadas usando uma ampla variedade de programas, tais como $S A S$ PROC MIXED (Littell et al., 1996), S-plus lme (Pinheiro e Bates, 2000), entre outros.

$\mathrm{O}$ modelo linear misto freqüentemente encontrado na literatura para respostas contínuas pode ser escrito na forma

$$
\mathrm{Y}_{i}=\mathrm{X}_{i} \boldsymbol{\beta}+\mathrm{Z}_{i} \mathbf{b}_{i}+\epsilon_{i}, \quad i=1, \ldots, m,
$$

em que $\mathbf{Y}_{i}$ de dimensão $\left(n_{i} \times 1\right)$ é um vetor de respostas contínuas observadas em uma unidade amostral $i, \mathrm{X}_{i}$ de dimensão $\left(n_{i} \times p\right)$ é a matriz de planejamento (conhecida e de posto completo) correspondente ao efeito fixo, $\boldsymbol{\beta}$ de dimensão $(p \times 1)$ é um vetor de coeficientes da regressão da média populacional de efeitos fixos, $\mathbf{Z}_{i}$ de dimensão $\left(n_{i} \times q\right)$ é a matriz de planejamento (conhecida e de posto completo) correspondente ao vetor de efeitos aleatórios $\mathbf{b}_{i}$, de dimensão $(q \times 1)$, e $\epsilon_{i}$ de dimensão $\left(n_{i} \times 1\right)$ é o vetor de erros aleatórios. Em geral, assume-se que os efeitos aleatórios $\mathbf{b}_{i}$ e os 
componentes residuais $\epsilon_{i}$ são independentes com

$$
\mathrm{b}_{i} \stackrel{i i d}{\sim} N_{q}(0, \mathrm{D}) \text { e } \epsilon_{i} \stackrel{i n d}{\sim} N_{n_{i}}\left(0, \Sigma_{i}\right)
$$

em que $\mathbf{D}=\mathbf{D}(\alpha)$ e $\Sigma_{i}=\Sigma_{i}(\gamma), i=1, \ldots, m$, são matrizes de dispersão de ordens $m q$ e $n=\sum_{i=1}^{m} n_{i}$, positivas definidas, que correspondem respectivamente, à variabilidade entre e intra-unidades amostrais sendo dependentes de um número desconhecido e reduzido de parâmetros $\alpha$ e $\gamma$, respectivamente. Dependendo do contexto, várias suposições podem ser feitas sobre as matrizes de covariâncias $\mathrm{D}$ e $\boldsymbol{\Sigma}_{i}, i=1, \ldots, m$. Diferentes estruturas para D e $\boldsymbol{\Sigma}_{i}$ podem ser encontradas na literatura; por exemplo, Verbeke e Molenberghs (2000) e Pinheiro e Bates (2000), entre outros. Neste trabalho, assumiremos que $\Sigma_{i}=\sigma_{\epsilon}^{2} \mathbf{I}_{n_{i}} i=1, \ldots, m$, neste contexto o modelo (2.12)-(2.13) é chamado de modelo de independência condicional.

\subsubsection{Modelos de análise de sobrevivência}

Nesta seção apresentamos uma introdução aos fundamentos de análise de sobrevivência. A análise sobrevivência consiste em uma relação de procedimentos estatísticos para a análise de dados relacionados ao tempo até a ocorrência de um determinado evento de interesse, a partir de um tempo inicial pré-estabelecido, que pode ser, por exemplo, o retorno de uma doença, a morte de um paciente, a falha de um item ou a realização de uma tarefa. Este tempo é geralmente referido como tempo de sobrevivência ou tempo de vida. Chamaremos as unidades de estudo de indivíduos, e o conjunto de observações de dados de sobrevivência. Um complicador presente nos dados de sobrevivência é a presença de observações censuradas, isto é, para alguns indivíduos o evento de interesse pode não ocorrer até o final do tempo de estudo. As censuras freqüentemente ocorrem porque o indivíduo pode abandonar o estudo, pode 
haver perda de contato ou pela limitação no tempo de acompanhamento do estudo. Dessa forma, existe a necessidade da inclusão de uma variável extra na análise, que indica se o tempo de sobrevivência de um determinado indivíduo foi ou não observado. A variável é conhecida na literatura de análise de sobrevivência e confiabilidade como variável indicadora de censura, ou simplesmente censura. Geralmente, os dados de sobrevivência apresentam censura à direita, ou seja, os verdadeiros tempos de vida são possivelmente maiores do que os valores observados. Além do tempo de sobrevivência e da variável indicadora de censura, também podemos observar nos dados variáveis que representam a heterogeneidade existente na população, tais como, idade, sexo, nível de colesterol, pressão arterial, entre outras, como também possíveis tratamentos aos quais os indivíduos são submetidos. Estas variáveis são conhecidas como variáveis explicativas ou variáveis regressoras de interesse.

\section{Conceitos básicos de análise de sobrevivência}

A seguir apresentamos a definição de três funções matematicamente equivalentes que podem ser utilizadas para caracterizar o comportamento de dados de tempo de sobrevivência. Seja $T$ uma variável aleatória contínua e positiva representando o tempo de sobrevivência, de modo que a distribuição de $T$ pode ser expressa através de várias funções matematicamente equivalentes tais que se uma delas é especificada, as outras podem ser obtidas. Entre elas temos a função de sobrevivência, $S(t)$, a função de densidade de probabilidade, $f(t)$, e a função de risco, $h(t)$, definidas, respectivamente, como

$$
S(t)=P(T \geq t)=1-F(t), \quad f(t)=\frac{d S(t)}{d t} \text { e } h(t)=\frac{f(t)}{S(t)}
$$

Algumas distribuições consideradas na literatura para o tempo de vida são as distribuições exponencial, Weibull, gama e log-normal. Propriedades destas distribuições 
podem ser estudas em Lawless (1982) e Cox e Oakes (1984), entre outras. Em nosso trabalho a distribuição log-normal é a que será considerada quando trabalhamos com dados de sobrevivência.

\section{Distribuição log-normal}

A distribuição log-normal, assim, como a distribuição de Weibull, tem sido muito utilizada em análise de dados de sobrevivência. Esta distribuição tem sido usada em diversas situações tais como análise de tempo de falha de isolação elétrica (Nelson e Hahn, 1972) e o estudo do tempo da presença de câncer de pulmão em fumantes (Whittemore e Altschuler, 1976). A distribuição é facilmente especificada, isto é, o tempo de vida $T$ segue a distribuição log-normal se o logaritmo do tempo de vida, $Z=\log T$, é distribuído normalmente com média $\mu$ e variância $\sigma^{2}$. A função de densidade de $T$ é escrita na forma (Klein e Moeschberger, 1997)

$$
f(t)=\frac{1}{\sqrt{2 \pi} \sigma t} \exp \left\{-\frac{1}{2}\left(\frac{\log t-\mu}{\sigma}\right)^{2}\right\} .
$$

Uma desvantagem da distribuição log-normal é, não apresentar função de sobrevivência e de risco analíticamente explícitas. A função de sobrevivência é escrita em termos da função de distribuição normal padrão, dada por

$$
S(t)=1-\Phi\left(\frac{\log t-\mu}{\sigma}\right) .
$$

Utilizando a relação $h(t)=\frac{f(t)}{S(t)}$, obtemos a função de risco. Mudholkar et al. (1996) apresentam alguns exemplos reais vinculados a estudos oncológicos, em que as funções de riscos apresentam este comportamento.

\section{Modelos de regressão}

Existem várias situações onde podemos observar nos indivíduos, além do tempo de sobrevivência e da variável indicadora de censuras, variáveis explicativas ou co- 
variáveis que representam parte da heterogeneidade existente na população, tais como, idade, sexo e pressão arterial, é denotadas por $\mathrm{x}=\left(x_{1}, \ldots, x_{p}\right)^{\top}$. Assumimos que a relação entre x e $T$ pode ser explicada através de um modelo de regressão. Duas classes de modelos de regressão que podem ser considerados são os modelos de riscos proporcionais e os modelos de locação e escala.

\section{Modelos de riscos proporcionais}

A família de modelos de riscos proporcionais é muita utilizada em análise de dados de sobrevivência e pode ser definida através da função de risco, expressa a seguir:

$$
h(t ; \mathbf{x})=h_{0}(t) \exp \left(\boldsymbol{\theta}^{\top} \mathbf{x}\right)
$$

sendo $\theta$ um vetor $p \times 1$ de coeficientes das covariáveis, e $h_{0}(t)$ a função risco para um indivíduo sob condições básicas $(x=0)$. A principal suposição considerada neste modelo é a proporcionalidade entre os riscos. Esta suposição pode ser exemplificada da seguinte forma: a razão entre duas funções de risco para dois indivíduos com covariáveis $\mathrm{x}_{1}$ e $\mathrm{x}_{2}$ não depende do tempo, isto é,

$$
\frac{h\left(t ; \mathbf{x}_{1}\right)}{h\left(t ; \mathbf{x}_{2}\right)}=\exp \left[\boldsymbol{\theta}^{\top}\left(\mathbf{x}_{1}-\mathbf{x}_{2}\right)\right]=r(\boldsymbol{\theta}) .
$$

Assim, pdemos dizer que o risco de falha, do primeiro indivíduo é $r(\boldsymbol{\theta})$ vezes o risco de falha do segundo indivíduo, para qualquer instante no tempo. Se não assumimos uma forma paramétrica particular para $h_{0}(t)$, obtemos o modelo de riscos proporcionais de Cox (Cox, 1972), que possui uma ampla literatura e extensões.

\section{Modelo de locação e escala}

A segunda classe importante de modelos é a classe de modelos de locação e escala sendo também chamado de modelo de tempo de falha acelerado (Keinding et al., 1997). Este modelo se caracteriza pelo fato de $Z=\log T$ ter uma distribuição com parâmetro 
de locação $\mu(\mathrm{x})$ dependendo do vetor de covariáveis $\mathrm{x}$, e um parâmetro de escala $\sigma$, constante. Geralmente assumimos $\mu(\mathrm{x})=\boldsymbol{\beta}^{\top} \mathrm{x}$, sendo $\boldsymbol{\beta}=\left(\beta_{0}, \beta_{1}, \ldots, \beta_{p-1}\right)^{\top}$ o vetor de parâmetros desconhecidos (consideramos que a primeira covariável toma o valor 1 para permitir a estimação do termo constante). Esta classe pode ser representada através da relação

$$
\log T=\boldsymbol{\beta}^{\top} \mathbf{x}+\sigma \epsilon
$$

$\operatorname{com} \sigma>0$, sendo $\epsilon$ um erro aleatório cuja distribuição não depende de $\mathbf{x}$. Desta forma temos um modelo de regressão linear em $Z$. Uma característica deste modelo é que as covariáveis atuam multiplicativamente sobre T. Lawless (1982) apresenta descrições mais detalhadas desta classe de modelos, assim como vários modelos paramétricos comumente usados. Consideramos a extensão estrutural deste modelo, ou seja, $x$ é observado através de $w=x+\xi$, onde $\xi$ é o erro de medição.

\section{Modelo de fragilidade}

Quando as observações pertencentes a um mesmo grupo apresentam-se correlacionadas, os modelos tradicionais para análise de sobrevivência como o popular modelo de riscos proporcionais de Cox ou o modelo de tempo de falha acelerado, descrito acima, não podem ser diretamente aplicados. Diversos autores estudam o uso dos chamados modelos de fragilidade, que representam uma generalização dos modelos de riscos proporcionais para tratar da heterogeneidade dos dados. Neste caso podemos, por exemplo, estar interessados em estudar o tempo até a ocorrência de alguma doença em ratos de uma mesma ninhada, ou em indivíduos de uma mesma família ou pacientes tratados em um mesmo hospital. Nessas situações é possível evidenciar a existência de correlação entre observações, quando os dados encontram-se com alguma estrutura de grupos. Esta heterogeneidade pode estar sendo incorporada pela presença de covariáveis envolvidas no estudo. Uma forma de tratar a hetero- 
geneidade dos dados é assumir que o efeito conjunto das covariáveis, cuja missão seria proporcionar a dependência entre indivíduos, poderia ser capturada por uma variável aleatória não observável (efeito aleatório), adicionada ao modelo para cada grupo de indivíduos. Seguindo esta abordagem, Vaupel et al. (1979), em um estudo de dados demográficos cria o termo fragilidade para designar o efeito aleatório e desenvolve uma versão particular de um modelo para tratar dados correlacionados, estendendo o modelo de riscos proporcionais. Este modelo foi estudado e ampliado por diversos autores. Resultados importantes podem ser encontrados, por exemplo, em Hougaard (1987), Oakes (1989) e Klein (1992). Uma ampla revisão sobre inferência em modelos de fragilidade pode ser vista em Hougaard (2000). Nosso objetivo é a modelagem do tempo de falha através da distribuição log-normal, onde observamos uma covariável medida com erros. Desenvolvemos estimação por máxima verossimilhança para os efeitos aleatórios. Um estudo de simulação ilustra o viés incorrido pelo estimador ingênuo ("naive") e a correspondente correção pelo enfoque desenvolvido, levando em conta diferentes índices de censura.

\subsection{Distribuição normal-assimétrica}

A distribuição normal-assimétrica univariada surgiu independentemente em vários artigos estatísticos, entre os quais pode-se destacar Roberts (1966), O'Hagan e Leonard (1976) e Aigner et al. (1977). Entretanto, foi Azzalini (1985) quem apresentou formalmente esta distribuição e estudou as suas propriedades. Generalizações dessas idéias para o caso multivariado tem sido propostas por vários autores, como por exemplo, Azzalini e Dalla Valle (1996), Azzalini e Capitanio (1999), Sahu et al. (2003), Genton et al. (2001) e Genton (2004). 


\subsubsection{Distribuição normal-assimétrica univariada de Azzalini}

Nesta subseção definimos a distribuição normal-assimétrica univariada e apresentamos algumas propriedades desta distribuição.

Definição 2.1. Uma variável aleatória $Z$ tem distribuição normal-assimétrica padrão com parâmetro de assimetria $\lambda \in \mathbb{R}$ denotada por $S N(\lambda)$, se sua função densidade de probabilidade é dada por

$$
f_{Z}(z)=2 \phi(z) \Phi(\lambda z), \quad z \in \mathbb{R}
$$

onde $\phi($.$) e \Phi($.$) são as funções densidade de probabilidade (fdp) e de distribuição$ acumulada (fda) de uma distribuiçã் normal padrão, respectivamente.

A densidade em (2.14) é uma densidade válida para $\lambda \in \mathbb{R}$ e é simétrica se, e somente se, $\lambda=0$ sendo que neste caso a densidade coincide com a densidade da distribuição normal padrão, e portanto é simétrica. Valores negativos de $\lambda$ implicam assimetria negativa e valores positivos de $\lambda$, assimetria positiva. Utilizaremos a seguinte notação $Z \sim S N(\lambda)$. A figura (2.1) ilustra o comportamento desta densidade para alguns valores de $\lambda$.

Listamos a seguir algumas propriedades interessantes da distribuição normalassimétrica padrão:

(P1) $E[Z]=\sqrt{\frac{2}{\pi}} \frac{\lambda}{\sqrt{1+\lambda^{2}}}$ e $\operatorname{Var}[Z]=1-\frac{2}{\pi} \frac{\lambda^{2}}{1+\lambda^{2}}$.

(P2) Os momentos de ordem par de $Z$ coincidem com os da distribuição normalpadrão, isto é,

$$
E\left[Z^{2 k}\right]=2^{-k} \frac{(2 k) !}{k !}
$$


Figura 2.1: Função de densidade da normal-assimétrica para $\lambda=-5,-3,0,3,5$.

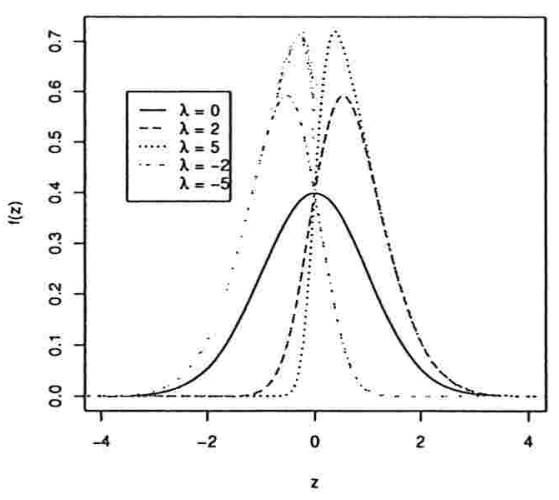

Os momentos ímpares são dados por

$$
E\left[Z^{2 k+1}\right]=\sqrt{\frac{2}{\pi}} \lambda\left(1+\lambda^{2}\right)^{-\left(k+\frac{1}{2}\right)} 2^{-k}[(2 k+1) !] \sum_{j=0}^{k} \frac{j !(2 \lambda)^{2 j}}{(j+1) !(k-j) !} .
$$

(P3) O coeficiente de assimetria é dado por

$$
\gamma=\sqrt{\frac{2}{\pi}}\left(\frac{4}{\pi}-1\right)\left(\frac{\lambda}{\sqrt{1+\lambda^{2}}}\right)^{3}\left(1-\frac{2}{\pi} \frac{\lambda^{2}}{1+\lambda^{2}}\right)^{-3 / 2}
$$

e o coeficiente de curtose é expresso por

$$
\kappa=\frac{8}{\pi^{3}}(\pi-3)\left(\frac{\lambda}{\sqrt{1+\lambda^{2}}}\right)^{4}\left(1-\frac{2}{\pi} \frac{\lambda^{2}}{1+\lambda^{2}}\right)^{-2} .
$$

(P4) Se $Z \sim S N(\lambda)$, então $|Z| \sim H N(0,1)$.

(P5) Quando $\lambda \rightarrow \infty$, a densidade (2.14) converge para a densidade de uma distribuição $H N(0,1)$.

(P6) Se $Z \sim S N(\lambda)$, então $-Z \sim S N(-\lambda)$.

(P7) Se $Z \sim S N(\lambda)$, então $Z^{2} \sim \chi_{1}^{2}$. 
onde denotamos por $H N(0,1)$ a distribuição half-normal (ou normal truncada a esquerda do zero) que tem densidade dada por $2 \phi(z) I_{[0, \infty)}(z)$. Outras propriedades e as demonstrações destas podem ser encontradas em Azzalini (1985), Azzalini (2005). Ver também Bayes (2005), entre outros.

Uma forma de apresentar a distribuição normal-assimétrica é através da representação estocástica, à qual foi obtida por Henze (1986), e cujo resultado é dado pela proposição 2.1 definida a seguir.

Proposição 2.1. Sejam $Y_{1}$ e $Y_{2}$ variáveis aleatórias independentes com distribuição normal padrão. Então, $Z \sim S N(\lambda)$ é tal que

$$
Z \stackrel{d}{=} \delta\left|Y_{1}\right|+\sqrt{1-\delta^{2}} Y_{2}, \operatorname{com} \delta=\frac{\lambda}{\sqrt{1+\lambda^{2}}},
$$

onde $\stackrel{d}{=}$ significa "distribuído como".

Demonstração. Ver Bayes (2005).

\subsubsection{Distribuição normal-assimétrica de três parâmetros}

Na seguinte definição estendemos o modelo (2.14) incluindo parâmetros de locação $(\mu \in \mathbb{R})$ e escala $(\sigma>0)$. Neste caso, utilizaremos a notação $Y \sim S N\left(\mu, \sigma^{2}, \lambda\right)$.

Definição 2.2. Uma variável aleatória $Y$ tem distribuição normal-assimétrica com parâmetros de posição $\mu$ e de escala $\sigma$ se sua função de densidade de probabilidade é da forma

$$
f_{Y}(y)=\frac{2}{\sigma} \phi\left(\frac{y-\mu}{\sigma}\right) \Phi\left(\lambda \frac{y-\mu}{\sigma}\right), y \in \mathbb{R}
$$

É fácil verificar que se $Z \sim S N(\lambda)$ e $Y=\mu+\sigma Z$, então $Y \sim S N\left(\mu, \sigma^{2}, \lambda\right)$. 


\subsubsection{Distribuição normal-assimétrica multivariada de La- chos}

Nesta subseção consideramos a definição proposta por Lachos (2004), que unifica as definições encontradas em Azzalini e Dalla Valle (1996), Azzalini e Capitanio (1999) e Arellano-Valle e Genton (2005).

Definição 2.3. Um vetor aleatório n-dimensional $\mathbf{Y}$ segue uma distribuição normalassimétrica com vetor de locação $\mu \in \mathbb{R}^{n}$, matriz de dispersão $\Sigma$ (uma matriz $n \times n$ definida positiva) e vetor de assimetria $\lambda \in \mathbb{R}^{n}$, denotada por $\mathrm{Y} \sim S N_{n}(\boldsymbol{\mu}, \Sigma, \lambda)$, se sua fdp é dada por

$$
f_{\mathrm{Y}}(\mathrm{y})=2 \phi_{n}(\mathrm{y} \mid \boldsymbol{\mu}, \boldsymbol{\Sigma}) \Phi\left(\lambda^{\top} \Sigma^{-1 / 2}(\mathrm{y}-\mu)\right), \quad \mathrm{y} \in \mathbb{R}^{n}
$$

onde $\phi_{n}(. ; \boldsymbol{\mu}, \boldsymbol{\Sigma})$ e $\Phi(. ; \boldsymbol{\mu}, \boldsymbol{\Sigma})$ denotam a fdp e a fda, respectivamente, da distribuição $N_{n}(\boldsymbol{\mu}, \boldsymbol{\Sigma})$. Quando $\boldsymbol{\mu}=0$ e $\boldsymbol{\Sigma}=\mathbf{I}_{n}$, uma matriz indentidade de dimensão $(n \times n)$, temos

$$
f_{\mathbf{Y}}(\mathbf{y})=2 \phi_{n}(\mathbf{y}) \Phi\left(\boldsymbol{\lambda}^{\top} \mathbf{y}\right), \quad \mathrm{y} \in \mathbb{R}^{n}
$$

e neste caso denotamos por $\mathrm{Y} \sim S N_{n}(\lambda)$.

É importante notar que a respresentação estocástica apresentada em (2.15) é um caso especial da representação estocástica dada na seguinte proposição:

Proposição 2.2. Seja $\mathrm{Y} \sim S N_{n}(\boldsymbol{\mu}, \boldsymbol{\Sigma}, \lambda)$. Então, $\mathrm{Y}$ pode ser representada estocasticamente por

$$
\mathrm{Y} \stackrel{d}{=} \mu+\Sigma^{1 / 2}\left(\delta\left|X_{0}\right|+\left(\mathrm{I}_{n}-\delta \delta^{\top}\right)^{1 / 2} \mathrm{X}_{1}\right), \operatorname{com} \delta=\frac{\lambda}{\left(1+\delta^{\top} \delta\right)^{1 / 2}}
$$

onde $X_{0} \sim N_{1}(0,1)$ e $\mathbf{X}_{1} \sim N_{n}\left(\mathbf{0}, \mathbf{I}_{n}\right)$ são independentes. 
Demonstração. Ver Arellano-Valle e Genton (2005) e também Lachos (2004).

Esta representação estocástica será de grande utilidade para a inferência estatística nos Capítulos 3 e 4.

Definimos a seguir uma nova distribuição normal-assimétrica univariada proposta por Sahu et al. (2003) e apresentamos também a extensão multivariada desta distribuição. A vantagem da utilização desta distribuição é no sentido de obter algoritmos eficientes para a obtenção de estimativas de máxima verossimilhança.

\subsubsection{Distribuição normal-assimétrica univariada de Sahu}

Nesta subseção definimos a distribuição normal-assimétrica univariada de Sahu et al. (2003) e apresentamos algumas propriedades desta distribuição.

Definição 2.4. Uma variável aleatória $Y$ segue uma distribuição normal-assimétrica com parâmetro de locação $\mu$, parâmetro de escala $\sigma^{2}$ e parâmetro de assimetria $\lambda$, se sua função de densidade de probabilidade é da forma

$$
f_{Y}(y)=2 \phi_{1}\left(y \mid \mu, \sigma^{2}+\lambda^{2}\right) \Phi_{1}\left(\frac{\lambda}{\sigma} \frac{(y-\mu)}{\left(\sigma^{2}+\lambda^{2}\right)^{1 / 2}}\right) .
$$

em que $\phi\left(. \mid a, b^{2}\right)$ e $\Phi\left(. \mid a, b^{2}\right)$ denotam, respectivamente, a função de densidade de probabilidade (fdp) e a função de distribuição acumulada (fda) da $N\left(a, b^{2}\right)$ e quando $a=0$ e $b=1$ denotamos esta função por $\phi($.$) e \Phi($.$) , respectivamente. Usamos$ a notação $Y \sim S N S\left(\mu, \sigma^{2}, \lambda\right)$ para denotar esta classe de distribuições, que será reduzida para $S N S(\lambda)$ quando tivermos $\mu=0$ e $\sigma^{2}=0$.

È importante ressaltar que se considerarmos que $Y \sim S N S\left(\mu, \sigma^{2}, \lambda\right)$ é equivalente obter que $Y \sim S N\left(\mu, \sigma^{2}+\lambda^{2}, \lambda / \sigma\right)$. Note que uma vantagem de trabalhar com a 
distribuição normal-assimétrica de Sahu et al. é obter estimativas de $\lambda$ finitas.

Algumas propriedades importantes da variável aleatória $Y \sim S N S\left(\mu, \sigma^{2}, \lambda\right)$ são apresentadas a seguir:

$$
E[Y]=\mu+\sqrt{\frac{2}{\pi}} \lambda, \quad \text { e } \quad \operatorname{Var}[Y]=\sigma^{2}+\left(1-\frac{2}{\pi}\right) \lambda^{2}
$$

(P2) Os momentos de ordem par de $Y$ é dado por

$$
E\left[Y^{2 k}\right]=\left(1+\lambda^{2}\right)^{k} \frac{(2 k-1) !}{2^{k-1}(k-1) !} .
$$

Os momentos ímpares são dados por

$$
E\left[Y^{2 k+1}\right]=\sqrt{\frac{2}{\pi}} \lambda \frac{(2 k+1) !}{2^{k}} \sum_{j=0}^{k} \frac{j !(2 \lambda)^{(2 k)}}{(2 j+1) !(k-j) !}, k \geq 1 .
$$

(P3) O coeficiente de assimetria é dado por

$$
\gamma=\lambda^{3} \sqrt{\frac{2}{\pi}}\left(\frac{4}{\pi}-1\right)\left\{\sigma^{2}+\left(1-\frac{2}{\pi}\right) \lambda^{2}\right\}^{-3 / 2},
$$

e o coeficiente de curtose é expresso por

$$
\kappa=8 \lambda^{4} \frac{(\pi-3)}{\pi^{2}}\left\{\sigma^{2}+\left(1-\frac{2}{\pi}\right) \lambda^{2}\right\}^{-2} .
$$

(P4) $-Y \sim S N S\left(\mu, \sigma^{2},-\lambda\right)$.

(P5) $Y \sim S N S\left(\mu, \sigma^{2}, \lambda\right)$ se, e somente se, $Y \sim S N\left(\mu, \sigma^{2}+\lambda^{2}, \lambda / \sigma\right)$.

A representação estocástica proposta pelos autores é útil para estudar algumas propriedades e também para obter simulações de uma variável aleatória que segue uma distribuição $S N S\left(\mu, \sigma^{2}, \lambda\right)$. Esta representação é expressa por

$$
Y \stackrel{d}{=} \lambda\left|X_{o}\right|+X_{1}
$$

em que $X_{o} \sim N(0,1)$ independente de $X_{1} \sim N\left(\mu, \sigma^{2}\right)$ e $\stackrel{d}{=}$ " significa "distribuido como". A distribuição $S N S$ é unimodal e se $\lambda<0$, a distribuição é assimétrica à esquerda e se $\lambda>0$, assimétrica à direita. 


\subsubsection{Distribuição normal-assimétrica multivariada de Sahu}

Nesta seção consideramos a extensão univariada proposta por Sahu et al. (2003). A definição da distribuição normal-assimétrica multivariada é dada a seguir.

Definição 2.5. Um vetor aleatório n-dimensional $\mathrm{Y}$ segue uma distribuição normalassimétrica com vetor de locação $\boldsymbol{\mu} \in \mathbb{R}^{n}$, matriz de dispersão $\boldsymbol{\Sigma}$ (uma matriz $n \times n$ definida positiva) e o vetor de assimetria $\lambda \in \mathbb{R}^{n}$, se sua fdp é dada por

$$
f_{\mathrm{Y}}(\mathrm{y})=2 \phi_{n}\left(\mathrm{y} \mid \boldsymbol{\mu}, \Sigma+\lambda \lambda^{\top}\right) \Phi_{1}\left(\frac{\lambda^{\top} \Sigma^{-1}(\mathrm{y}-\mu)}{\sqrt{1+\lambda^{\top} \Sigma^{-1} \lambda}}\right), \quad \mathrm{y} \in \mathbb{R}^{n},
$$

denotada por $\mathbf{Y} \sim S N S_{n}(\boldsymbol{\mu}, \boldsymbol{\Sigma}, \boldsymbol{\lambda})$. Quando $\boldsymbol{\mu}=0$ e $\Sigma=\mathbf{I}_{n}$, a matriz identidade de dimensão $(n \times n)$, temos

$$
f_{\mathbf{Y}}(\mathbf{y})=2 \phi_{n}(\mathbf{y}) \Phi\left(\boldsymbol{\lambda}^{\top} \mathbf{y}\right), \quad \mathrm{y} \in \mathbb{R}^{n}
$$

e neste caso denotamos por $\mathrm{Y} \sim S N S_{n}(\boldsymbol{\lambda})$.

Note que a densidade (2.20) não se encontra na classe de distribuições assimétricas multivariadas definidas por Sahu et al. (2003) dado que nesse trabalho a função de assimetria é de dimensão $n$. Entretanto, a densidade (2.20) pertence à classe de distribução normal-assimétrica fundamental definida por Arellano-Valle e Genton (2005). Assim, várias propriedades da distribuição normal-assimétrica podem ser obtidas a partir de resultados desenvolvidos por Arellano-Valle e Genton (2005). A partir disto, por exemplo, segue que a representação estocástica para um vetor aleatório normal-assimétrico multivariado é dada conforme abaixo.

Proposição 2.3. Seja $\mathrm{Y} \sim S N S_{n}(\boldsymbol{\mu}, \boldsymbol{\Sigma}, \boldsymbol{\lambda})$. Então,

$$
\mathrm{Y} \stackrel{d}{=} \lambda\left|X_{0}\right|+\mathrm{X}_{1}
$$

em que $X_{0} \sim N(0,1)$ independe de $\mathrm{X}_{1} \sim N_{n}(\boldsymbol{\mu}, \boldsymbol{\Sigma})$. 
Note que a representação estocástica dada em (2.19) para o caso univariado é um caso especial de (2.21). Assim, estendemos a distribuição normal-assimétrica apresentada em Sahu et al. (2003) para o caso multivariado. Na Figura 2.2 apresentamos alguns gráficos de contornos da densidade normal-assimétrica bivariada $S N S_{2}(0, \Sigma, \lambda)$ para diferentes valores de $\Sigma$ e $\lambda$, onde $\Sigma=\mathrm{I}-\boldsymbol{\lambda} \boldsymbol{\lambda}^{\top}$. Note que o gráfico de contornos podem ser fortemente assimétricos dependendo da escolha dos parâmetros.

Figura 2.2: Contornos da distribuição normal-assimétrica bivariada para diferentes valores de $\lambda$ : (a) $\lambda_{1}=0$ e $\lambda_{2}=0$, (b) $\lambda_{1}=-0.7$ e $\lambda_{2}=0.9$, (c) $\lambda_{1}=0.9$ e $\lambda_{2}=-0.7$ e (d) $\lambda_{1}=0.2$ e $\lambda_{2}=0.9$.

(a)

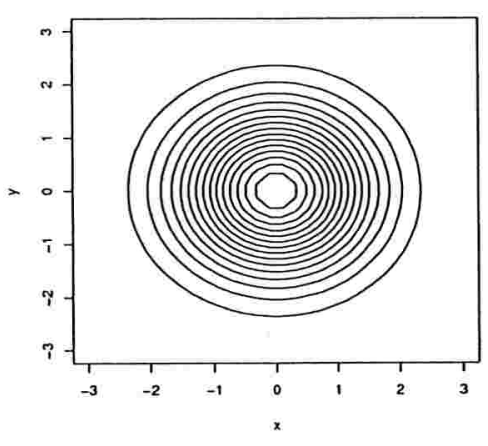

(c)

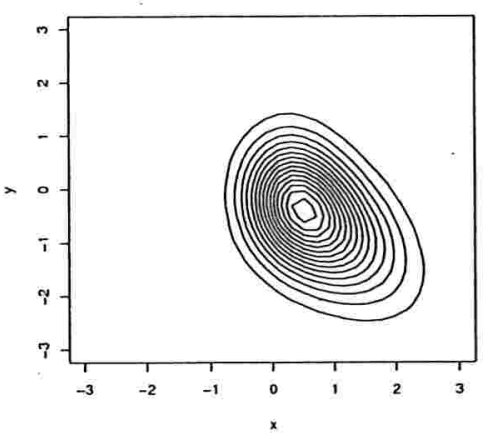

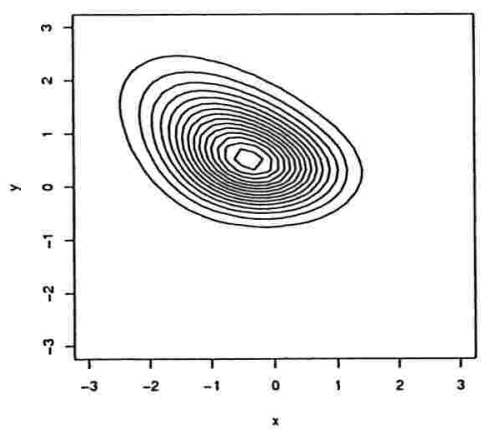

(d)

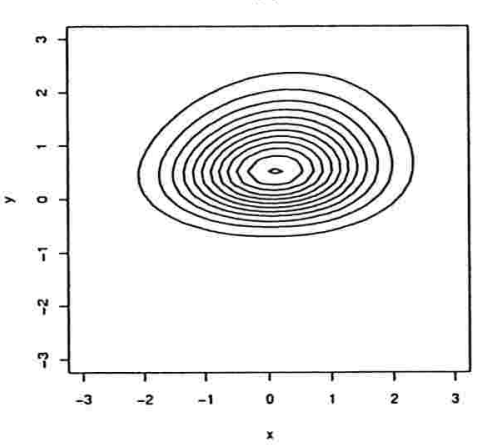

Uma consequência direta da Proposição 2.3 é apresentada na seguinte proposição.

Proposição 2.4. Seja $\mathrm{Y} \sim S N_{n}(\boldsymbol{\mu}, \boldsymbol{\Sigma}, \boldsymbol{\lambda})$. Então, 
i) $\mu_{1}=E[Y]=\mu+\sqrt{\frac{2}{\pi}} \lambda$.

ii) $\mu_{2}=E\left[Y Y^{\top}\right]=\Sigma+\lambda \lambda^{\top}+\sqrt{\frac{2}{\pi}}\left(\mu \lambda^{\top}+\lambda \mu^{\top}\right)$, $\operatorname{Var}[Y]=\Sigma+\left(1-\frac{2}{\pi}\right) \lambda \lambda^{\top}$.

\subsection{Algoritmo EM}

A máximização direta da função de log-verossimilhança através do método de quaseNewton (BFGS implementado no MATLAB e L-BFGS-B no R) algumas vezes pode dificultar quando o termo $\log \Phi(a)$ encontrado na função de log-verossimilhança pode produzir problemas computacionais quando $a$ seja negativo $(a<-38$ por exemplo). Além disso, este método não parece robusto em relação a valores iniciais, a menos que, os valores iniciais sejam próximos do ponto de máximo. Assim, a abordagem da maximização direta geralmente não converge.

O algoritmo EM (Dempster et al., 1977) é um algoritmo iterativo muito utilizado para a estimação de máxima verossimilhança em modelos com dados incompletos. Mais especificamente, denotamos por $\mathbf{y}$ os dados observados e $\mathbf{s}$ os dados faltantes. Os dados completos são denotados por $\mathrm{z}=(\mathrm{y}, \mathrm{s})$, sendo $\mathrm{y}$ aumentado com $\mathrm{s}$, considerados dados faltantes. A função de log-verossimilhança dos dados completos é denotada por $\ell_{c}(\boldsymbol{\theta} \mid \mathrm{y}, \mathrm{s}), \boldsymbol{\theta} \in \Theta$, e $Q\left(\boldsymbol{\theta}, \boldsymbol{\theta}^{(m-1)}\right)$ denota o valor esperado de $\ell_{c}$, e os atuais parâmetros estimados. Temos que

$$
Q\left(\boldsymbol{\theta}, \boldsymbol{\theta}^{(m-1)}\right)=E\left[\ell_{c}(\boldsymbol{\theta} \mid \mathbf{y}, \mathrm{s}) \mid \mathbf{y}, \boldsymbol{\theta}^{(m-1)}\right],
$$

em que $\theta^{(m-1)}$ são os atuais parâmetros estimados que são usados para avaliar a esperança e $\boldsymbol{\theta}$, são estimativas atualizadas, obtidas por maximização de $Q$. 
Cada iteração do algoritmo EM envolve dois passos. O primeiro passo refere-se ao cálculo da esperança condicional e o segundo, à maximização, ou seja, Passo-E: Calcular $Q\left(\boldsymbol{\theta}, \boldsymbol{\theta}^{(m-1)}\right)$ como uma função de $\boldsymbol{\theta}$;

Passo-M: Encontrar $\boldsymbol{\theta}^{(m)}$, tal que, $Q\left(\boldsymbol{\theta}^{(m)}, \boldsymbol{\theta}^{(m-1)}\right)=\max _{\boldsymbol{\theta} \in \Theta} Q\left(\boldsymbol{\theta}, \boldsymbol{\theta}^{(m-1)}\right)$. Assim, os dois passos são repetidos o quanto seja necessário. Cada iteração do algoritmo EM aumenta o valor da função de log-verossimilhança $\ell(\boldsymbol{\theta})$ e, geralmente, converge a um máximo local ou global da função de log-verossimilhança.

Portanto, para verificar-se que o máximo verdadeiro é identificado, geralmente é recomendado rodar várias vezes as iterações do algoritmo EM com diferentes valores iniciais.

\subsection{Critérios de informação}

Para a selecção dos modelos podemos utilizar alguns critérios de informação fundamentados na teoria da decisão que penalizam os modelos com um grande número de parâmetros. Os três critérios de informação selecionados são baseados nas seguintes estatísticas AIC (Akaike Information Criterion), o BIC (Bayesian Information Criterio) e o HQ (Hannan-Quinn Criterion) definidos como

$$
\begin{aligned}
A I C & =-\ell(\widehat{\boldsymbol{\theta}})+P, \\
B I C & =-\ell(\widehat{\boldsymbol{\theta}})+0.5 \log (N) P \\
H Q & =-\ell(\widehat{\boldsymbol{\theta}})+\log (\log (N)) P,
\end{aligned}
$$

$\operatorname{com} \ell(\widehat{\theta})$ representando o máximo da log-verossimilhança, $P$ é o número de parâmetros do modelo e $N$ o número de observações. Quanto menor for o valor dessas estatísticas, maior evidência favorável ao modelo em estudo. 


\subsection{Influência local}

Dentre as abordagens mais utilizadas na prática, para medir influência em modelos de regressão, destacam-se as análises baseadas em influência local por Cook (1986) e aquelas obtidas via eliminação de observações (influência global). Adicionalmente, apresentamos a abordagen de Zhu e Lee (2001) baseada na função Q.

\subsubsection{Abordagem de Cox}

Sabemos que a detecção de observações influentes é um passo importante na análise de um conjunto de dados. Existem várias alternativas para avaliar a influência dos dados e/ou modelo perturbado sobre os estimadores dos parâmetros. Por exemplo, ver Cook e Weisberg (1982) e Chatterjee e Hadi (1988).

Eliminação de observações é comum quando pretendemos avaliar o efeito de uma observação sob o processo de estimação. A análise de influência global a partir do efeito de observações é avaliado por eliminar esta observação do conjunto de dados. Alternativamente, influência local proposto por Cook (1986) é baseada em avaliar a mudança nos resultados da análise quando incorporamos "pequenas perturbações" ao modelo. Dentro desse contexto, podemos perturbar a variável resposta, a variável explicativa, a matrix de covariâncias, etc. A abordagem original é baseada na análise do afastamento da verossimilhança ("likelihood displacement") (ver Cook e Weisberg, 1982 e Cook, 1986)

$$
L D(\boldsymbol{\omega})=2\left\{l(\widehat{\boldsymbol{\theta}})-l\left(\widehat{\boldsymbol{\theta}}_{\omega}\right)\right\}
$$

em que $l(\theta)$ é a função de log-verossimilhança do modelo postulado, $\theta \in \mathbb{R}^{k}$ vetor de parâmetros desconhecidos, $l(\boldsymbol{\theta} \mid \omega)$ é a log-verossimilhança do modelo perturbado, $\boldsymbol{\omega}$ 
é um vetor de perturbações de dimensão $q \times 1$ em um subconjunto aberto $\Omega \subset \mathbb{R}^{q}$. O vetor representando ausência de perturbação é denotado por $\omega_{0} \subset \Omega$, ou seja, $l\left(\boldsymbol{\theta} \mid \omega_{0}\right)=l(\boldsymbol{\theta}), \forall \boldsymbol{\theta} \in \Theta$ e que $l(\boldsymbol{\theta} \mid \omega)$ possua as duas primeiras derivadas contínuas na vizinhança de $\left(\widehat{\boldsymbol{\theta}}^{\top}, \boldsymbol{\omega}_{0}^{\top}\right)$. $(\widehat{\boldsymbol{\theta}})$ e $\widehat{\boldsymbol{\theta}}_{\omega}$ denotam respectivamente, os EMV baseados em $(l(\boldsymbol{\theta}))$ e $l(\boldsymbol{\theta} \mid \boldsymbol{\omega})$. A idéia de influência local (Cook, 1986) consiste em caracterizar o comportamento de $L D(\boldsymbol{\omega})$ em $\omega_{0}$. O procedimento consiste em selecionar uma direção unitária $\boldsymbol{d},\|\boldsymbol{d}\|=1$, e então, considerar o gráfico de $L D\left(\boldsymbol{\omega}_{0}+a \boldsymbol{d}\right)$ contra $a \in$ $\mathbb{R}$. Este gráfico é chamado linha projetada. Note que $L D\left(\omega_{0}\right)=0$, ou seja, $L D\left(\omega_{0}+\right.$ ad) tem um mínimo local em $a=0$. Cada linha ajustada pode ser caracterizada pela curvatura normal $C_{d}(\boldsymbol{\theta})$ em torno de $a=0$. A sugestão é considerar a direção $d_{\max }$ correspondente à maior curvatura $C_{d \max }(\theta)$. O gráfico de índice de $d_{\max }$ pode mostrar aquelas observações que sob pequenas perturbações exercem uma notável influência sobre $L D(\omega)$. Cook (1986) mostrou que a curvatura normal na direção de $d$ toma a forma

$$
C_{\mathrm{l}}(\boldsymbol{\theta})=2\left|\boldsymbol{d}^{\top} \Delta^{\top} \mathbf{L}^{-1} \Delta d\right|
$$

em que $-\mathbf{L}=\frac{\partial^{2} \ell(\boldsymbol{\theta})}{\partial \boldsymbol{\theta} \partial \boldsymbol{\theta}^{\top}}$ é a matriz de informação de Fisher observada para o modelo $\left(\boldsymbol{\omega}=\omega_{0}\right)$ e $\Delta$ é a matriz de dimensão $k \times q$ com elementos

$$
\Delta_{r s}=\frac{\partial^{2} \ell(\boldsymbol{\theta} \mid \boldsymbol{\omega})}{\partial \theta_{r} \partial \omega_{s}}
$$

avaliadas em $\boldsymbol{\theta}=\widehat{\boldsymbol{\theta}}$ e $\boldsymbol{\omega}=\boldsymbol{\omega}_{0}, r=1, \ldots, k$ e $s=1, \ldots, q$. Portanto, a maximização de (2.22) é equivalente a encontrar o maior autovalor absoluto $C_{d \max }$ da matriz $\mathbf{B}=$ $-\Delta^{\top} L^{-1} \Delta$ e $d_{\max }$ é o autovetor normalizado correspondente.

Desta forma, $\boldsymbol{d}_{\max }$ pode ser utilizado como uma ferramenta útil na análise de diagnóstico. O gráfico dos elementos de $\left|d_{\max }\right|$ pode mostrar qual o tipo de perturbação tem a maior influência em $L D(\omega)$ em torno de $\omega_{0}$. Cook (1986) propõe examinar 
as componentes de $\boldsymbol{d}_{\max }$, independente do valor de $C_{d \max }$, uma vez que pode indicar observações que são conjuntamente influentes.

Em algumas situações é de interesse avaliar a influência sobre um subconjunto $\theta_{1}$ de $\boldsymbol{\theta}=\left(\boldsymbol{\theta}_{1}^{\top}, \boldsymbol{\theta}_{2}^{\top}\right)^{\top}$. Por exemplo, podemos ter interesse em $\boldsymbol{\theta}_{1}=\left(\mu_{x}, \boldsymbol{\beta}^{\top}\right)^{\top}$ ou em $\boldsymbol{\theta}_{1}=\lambda_{x}$. Em tais situações, a curvatura na direção $\boldsymbol{d}$ é dada por

$$
C_{l}\left(\boldsymbol{\theta}_{1}\right)=2\left|\boldsymbol{d}^{\top} \Delta^{\top}\left(\mathrm{L}^{-1}-\mathrm{B}_{22}\right) \Delta d\right|
$$

em que

$$
\mathbf{B}_{22}=\left(\begin{array}{cc}
0 & 0 \\
0 & L_{22}^{-1}
\end{array}\right),
$$

e $\mathbf{L}_{22}$ é obtido da partição de $\mathbf{L}$, segundo a partição de $\boldsymbol{\theta}$. O autovetor $\boldsymbol{d}_{\max }$ corresponde ao maior autovalor absoluto da matriz $\mathbf{B}=\Delta^{\top}\left(\mathbf{L}^{-1}-\mathbf{B}_{22}\right) \Delta$.

Escobar e Meeker (1992) (ver também Verbeke e Molenberghs, 2000) consideram que uma outra direção importante é dada por $d=e_{i n}$, um vetor de dimensão $n \times 1$ de zeros com um na $i$-ésima posição. Neste caso a curvatura normal, chamada de influência local total do indivíduo $i$, é dada por $C_{i}=2\left|e_{i n}^{\top} \mathrm{B} e_{i n}\right|=2\left|b_{i i}\right|$, em que $b_{i i}$ é o $i$-ésimo elemento diagonal de $\mathbf{B}, i=1, \ldots, n$.

Com a finalidade de comparar a influência local e global, utilizamos umas das medidas mais utilizadas para avaliar a influência de uma observação, via eliminação, é a distância de Cook $\left(D_{i}\right)$ (Cook e Weisberg, 1982) e o afastamento da verossimilhança $\left(L D_{i}\right)$ (Cook, 1977), definidas, respectivamente, por

$$
\begin{gathered}
D_{i}=\left(\widehat{\boldsymbol{\theta}}-\widehat{\boldsymbol{\theta}}_{(i)}\right)^{\top}(-\mathrm{L})\left(\widehat{\boldsymbol{\theta}}-\widehat{\boldsymbol{\theta}}_{(i)}\right) / k \\
L D_{i}=2\left[l(\widehat{\boldsymbol{\theta}})-l\left(\widehat{\boldsymbol{\theta}}_{(i)}\right)\right],
\end{gathered}
$$

em que $\widehat{\boldsymbol{\theta}}$ e $\widehat{\boldsymbol{\theta}}_{(i)}$ denota, respectivamente, a estimativa do vetor $\boldsymbol{\theta} \operatorname{com}$ todos os dados 
da amostra e com a eliminação da observação $i, i=1, \ldots, n$. Ver Zhao e Lee (1998) para mais detalhes.

\subsubsection{Abordagem de Zhu e Lee}

Nesta subseção desenvolvemos o método de influência local baseado na função Q de Zhu e Lee (2001) proveniente da esperança condicional da função de log-verossimilhança de dados completos do algoritmo EM. Motivada pelo trabalho dos autores, sabemos que em alguns casos fica difícil ou mesmo intratável aplicar diretamente os métodos apresentados por Cook $(1977,1986)$. No modelo linear misto normal-assimétrico multivariado é difícil calcular diretamente as estatísticas requeridas pelos métodos propostos por Cook $(1977,1986)$. Por esse fato, desenvolvemos as medidas de eliminação de observações e de influência local, baseadas na função Q definida a seguir

$$
Q(\boldsymbol{\theta}, \boldsymbol{\omega} \mid \widehat{\boldsymbol{\theta}})=E_{\mathbf{b}, \mathbf{T}}\left[\ell_{c}\left(\boldsymbol{\theta}, \boldsymbol{\omega} \mid \mathbf{Y}_{c}\right) \mid \mathbf{y}, \widehat{\boldsymbol{\theta}}\right]
$$

Seguindo as mesmas idéias de Cook (1986) continuamos considerando o vetor de perturbação $\boldsymbol{\omega}=\left(w_{1}, \ldots, w_{n}\right)^{\top}$ variando numa região aberta em $\Omega \subset \mathbb{R}^{n}$. Seja $\ell_{c}\left(\boldsymbol{\theta}, \boldsymbol{\omega} \mid \mathbf{Y}_{c}\right)$ a função de log-verossimilhança dos dados completos do modelo perturbado. Assumimos que existe um vetor de não perturbação $\omega_{o}$ tal que $\ell_{c}\left(\boldsymbol{\theta}, \boldsymbol{\omega}_{o} \mid \mathbf{Y}_{c}\right)=$ $\ell_{c}\left(\boldsymbol{\theta} \mid \mathbf{Y}_{c}\right)$ para todo $\boldsymbol{\theta}$. Denotamos $\widehat{\boldsymbol{\theta}}(\boldsymbol{\omega})$ como a função que maximiza $Q(\boldsymbol{\theta}, \boldsymbol{\omega} \mid \widehat{\boldsymbol{\theta}})=$ $E_{\mathbf{b}, \mathbf{T}}\left[\ell_{c}\left(\boldsymbol{\theta}, \omega \mid \mathbf{Y}_{c}\right) \mid \mathbf{y}, \widehat{\boldsymbol{\theta}}\right]$. O gráfico de influência é definido como $\boldsymbol{\alpha}(\boldsymbol{\omega})=\left(\boldsymbol{\omega}^{\top}, f_{Q}(\boldsymbol{\omega})\right)^{\top}$, onde $f_{Q}(\omega)$ é a função Q-afastamento definida por

$$
f_{Q}(\boldsymbol{\omega})=2\{Q(\widehat{\boldsymbol{\theta}} \mid \widehat{\boldsymbol{\theta}})-Q(\widehat{\boldsymbol{\theta}}(\boldsymbol{\omega}) \mid \widehat{\boldsymbol{\theta}})\}
$$

sendo $\widehat{\boldsymbol{\theta}}$ um ponto de mínimo local de $f_{Q}$. Seguindo a idéia de Cook (1986) e Zhu e Lee (2001) a curvatura normal $C_{f_{Q}, \mathrm{~d}}$, de $\boldsymbol{\alpha}(\boldsymbol{\omega})$ em $\omega_{o}$ na direção do vetor unitário d 
resume o comportamento de $f_{Q}$, definida

$$
C_{f_{Q}, \mathrm{~d}}=-2 \mathrm{~d}^{\top} \ddot{Q} \omega_{0} \mathrm{~d}, \quad-\ddot{Q} \omega_{o}=\Delta_{\omega_{0}}^{\top}\left\{-\ddot{Q}_{\theta}(\widehat{\theta})\right\}^{-1} \Delta_{\omega_{o}}
$$

em que $\ddot{Q}_{\boldsymbol{\theta}}(\widehat{\boldsymbol{\theta}})=\left.\frac{\partial^{2} Q(\boldsymbol{\theta} \mid \widehat{\boldsymbol{\theta}})}{\partial \boldsymbol{\theta} \partial \boldsymbol{\theta}^{\top}}\right|_{\boldsymbol{\theta}=\widehat{\boldsymbol{\theta}}}$ e $\Delta \boldsymbol{\omega}=\left.\frac{\partial^{2} Q(\boldsymbol{\theta}, \boldsymbol{\omega} \mid \widehat{\boldsymbol{\theta}})}{\partial \boldsymbol{\theta} \partial \boldsymbol{\omega}^{\top}}\right|_{\boldsymbol{\theta}=\hat{\boldsymbol{\theta}}(\boldsymbol{\omega})}$.

Como em Cook (1986), a matriz $-\ddot{Q} \omega_{\text {o }}$ é um elemento importante para detectar observações influentes. Uma descrição de $-\ddot{Q} \omega_{0}$, é dada por sua descomposição espectral

$$
-2 \ddot{Q} \omega_{\circ}=\sum_{m=1}^{p} \lambda_{m} \mathbf{e}_{m} \mathbf{e}_{m}^{\prime},
$$

em que $\left(\lambda_{1}, \mathrm{e}_{1}\right), \ldots,\left(\lambda_{1}, \mathrm{e}_{p}\right)$ são os pares de autovalores e autovetores da matriz $-\ddot{Q}_{\omega_{o}}$ com $\lambda_{1} \geq \ldots \geq \lambda_{p}, \lambda_{p+1}=\ldots=\lambda_{q}=0$ e $\mathrm{e}_{1}, \ldots, \mathrm{e}_{p}$ são elementos associados à base ortonormal. Assim, podemos expressar

$$
C_{f_{Q}, \mathbf{u}_{i}}=\sum_{m=1}^{p} \lambda_{m} e_{m l}^{2},
$$

onde $e_{m l}$ é o $l$-ésimo componente de $\mathbf{e}_{m} \in \mathbb{R}^{q}$ e $\mathbf{u}_{l}$ é um vetor coluna em $\mathbb{R}^{q} \operatorname{com}$ o $l$ ésimo componente igual a um, e todos os outros iguais a zero. De Cook (1986), $\mathbf{d}_{\max }=$ $\mathbf{e}_{1}$ fornece importantes informações para avaliar a influência de menor perturbação no modelo. Outra direção importante (ver Verbeke e Molenberghs, 2000) é $\boldsymbol{d}=\mathbf{u}_{l}$. Nesse caso, a curvatura normal é chamada de influência local total do l-ésimo indivíduo, a qual é dado por $C_{l}=2\left|\mathbf{u}_{l}^{\top} \ddot{Q} \omega_{o} \mathbf{u}_{l}\right|=2\left|b_{l l}\right|$, onde $b_{l l}$ é o $l$-ésimo elemento da matriz diagonal $\ddot{Q} \omega_{o}, l=1, \ldots, q$.

Em diagnóstico de eliminação de casos, é comum estudar os efeitos da eliminação da $i$-ésima observação de um conjunto de dados. As medidas clássicas como distância Cook e verossimilhança afastada são utilizados. Para nosso caso, desenvolvemos medidas de diagnóstico no vector eliminado $\left(\mathbf{y}_{i}^{\top}, \mathbf{x}_{i}^{\top}\right)$ e denotamos pelo índice $(i)$. 
Assi, temos que $\widehat{\boldsymbol{\theta}}_{(i)}$ é o máximo da função $Q_{(i)}(\boldsymbol{\theta} \mid \widehat{\boldsymbol{\theta}})$. De forma análoga, a distância de Cook para a função $Q(\boldsymbol{\theta} \mid \widehat{\boldsymbol{\theta}})$ é dado por

$$
D_{i}^{c}=\left(\widehat{\boldsymbol{\theta}}_{(i)}-\widehat{\boldsymbol{\theta}}\right)^{\top}(-\ddot{Q}(\widehat{\boldsymbol{\theta}} \mid \boldsymbol{\theta}))\left(\widehat{\boldsymbol{\theta}}_{(i)}-\widehat{\boldsymbol{\theta}}\right) /()
$$

de forma análoga a verossimilhança afastadade é expressa por

$$
L D_{i}^{c}=2\left[\ell_{c}(\boldsymbol{\theta})-\ell_{c}\left(\widehat{\boldsymbol{\theta}}_{(i)}\right)\right]
$$

onde $i=1, \ldots, n$, usamos $\boldsymbol{d}_{\max }$ e $C_{l}$ como influência local e $D_{i}^{c}, L D_{i}^{c}$ como influência global. Na seção seguinte, obtemos expressões analíticas para a matriz hessiana $\ddot{Q}_{\boldsymbol{\theta}}(\widehat{\theta})$ e a matriz $\Delta_{\boldsymbol{\omega}_{0}}$ sob diferentes esquemas de perturbação. 


\section{Capítulo 3}

\section{Modelo de Grubbs normal-assimé-}

\section{trico}

\subsection{Introdução}

O objetivo deste capítulo é apresentar um estudo do modelo estrutural de Grubbs sob a distribuição normal-assimétrica, onde é de interesse comparar vários instrumentos de medição que medem a mesma quantidade desconhecida $x$ em um grupo comum de $n$ unidades experimentais, supondo que as observações obtidas pelos diferentes instrumentos seguem uma distribuição normal-assimétria e a característica de interesse $x$ é medida na mesma escala. No contexto do modelo de Grubbs, a qualidade de um instrumento de medição é avaliada em termos da precisão relacionada com a proximidade de cada observação à sua própria média. Assim, a precisão está relacionada com a dimensão dos erros de medida, enquanto a acurácia mede a proximidade das médias ao verdadeiro valor. Desta forma, a acurácia está relacionada com os vícios. 
Grubbs $(1948,1973,1983)$ propôs um modelo para $n$ itens, cada item medido em $p$ instrumentos, dado por

$$
Y_{i j}=\alpha_{j}+x_{i}+\epsilon_{i j}
$$

onde $\alpha_{1}=0, Y_{i j}$ representa a medida do $i$-ésimo item no $j$-ésimo instrumento, $i=1, \ldots, n$ e $j=1, \ldots, p$. Geralmente na literatura assume-se que $x_{i}$ e $\epsilon_{i j}$ são independentes com distribuições normal $N_{1}\left(\mu_{x}, \phi_{x}\right)$ e $N_{1}\left(0, \phi_{j}\right)$, respectivamente. Este modelo pode ser visto como um caso especial do modelo com erro de medida multivariado (Fuller, 1987).

Embora a suposição de normalidade (ou simetria) seja utilizada em muitas situações, não é apropriada quando os dados apresentam um comportamento não-normal, tal como a assimetria. Os conjuntos de dados de Grubbs (1948) e Barnett (1969) apresentam este tipo de comportamento, ou seja, podem requerer transformação a fim de se aproximar melhor sua distribuição de uma distribuição normal. Azzalini e Dalla Valle (1996) fornecem várias razões para evitar este método (transformação de variáveis), se um modelo teórico mais apropriado pode ser encontrado. Com a finalidade de tratar tais problemas, neste trabalho assumiremos que a quantidade desconhecida $x$ segue uma distribuição normal-assimétrica, implicando que as quantidades observadas $\mathbf{Y}_{i}=\left(Y_{i 1}, \ldots, Y_{i p}\right)^{\top}$ têm distribuição normal-assimétrica multivariada definida anteriormente para a classe proposta por Arellano-Valle et al. (2005). No trabalho de de Castilho (2004) enfatiza-se que a distribuição de concentrações de uma substância no solo ou água, e de cobre ou ouro no solo, por exemplo, geralmente segue um comportamento assimétrico. Para a estimação dos parâmetros deste modelo usamos a estimação de máxima verossimilhança via algoritmo EM. Apresentamos matrizes apropriadas para avaliar a influência local de perturbações sobre os parâmetros estimados baseada em diferentes esquemas de perturbação. O nosso interesse neste capítulo é a comparação do ajuste do modelo de Grubbs estrutural normal com o 
modelo normal-assimétrico.

\subsection{Especificação do modelo}

Para especificar o modelo de Grubbs na classe normal-assimétrica, podemos expressar o modelo linear da seguinte forma:

$$
\mathrm{Y}_{i}=\mathbf{a}+\mathbf{1}_{p} x_{i}+\epsilon_{i}
$$

onde $\mathbf{a}=\left(0, \boldsymbol{\alpha}^{\top}\right)^{\top}=\left(0, \alpha_{2}, \ldots, \alpha_{p}\right)^{\top}$ e $\mathbf{1}_{p}=(1, \ldots, 1)^{\top}$ são vetores de dimensão $p \times 1 ; \mathbf{Y}_{i}=\left(Y_{i 1}, \ldots, Y_{i p}\right)^{\top}$ e $\epsilon_{i}=\left(\epsilon_{i 1}, \ldots, \epsilon_{i p}\right)^{\top}$ são vetores aleatórios de dimensão $p \times 1$, sendo este, chamado modelo de Grubbs sob a distribuição normal-assimétrica (MGSN), se

$$
\epsilon_{i} \stackrel{i i d}{\sim} N_{p}(0, D(\phi)) \text { e } x_{i} \stackrel{i i d}{\sim} S N_{1}\left(\mu_{x}, \phi_{x}, \lambda_{x}\right)
$$

$i=1, \ldots, n, \operatorname{com} D(\phi)=\operatorname{diag}\left(\phi_{1}, \ldots, \phi_{p}\right)^{\top}$ e $\phi=\left(\phi_{1}, \ldots, \phi_{p}\right)$, consideramos $\epsilon_{i}$ e $x_{i}$ mutuamente independentes. O modelo acima considera que a distribuição de $x$ representando a concentração de substâncias no solo ou água, sendo estas distribuídas assimétricamente na população. Por outro lado, os erros $\epsilon_{i}$, são relacionados aos erros de medida tal que eles são esperados serem distribuídos normalmente. O parâmetro de assimetria $\lambda_{x}$ expressa a assimetria na variável latente $x_{i}$ e, conseqüentemente, nas quantidades observadas $\mathbf{Y}_{i}, i=1, \ldots, n$, as quais, pode-se mostrar que tem, marginalmente, distribuição normal-assimétrica multivariada. Se $\lambda_{x}=0$, o modelo assimétrico reduz-se a um modelo de Grubbs normal (MGN), sendo o estudo inferencial extensivamente tratado na literatura. Note que a partir de (2.16), o modelo 
de regressão definido em (3.1)-(3.2) pode ser escrito de forma hierárquica, tal como

$$
\begin{aligned}
\mathrm{Y}_{i} \mid x_{i} & \stackrel{\text { ind }}{\sim} N_{p}\left(\mathbf{a}+\mathbf{1}_{p} x_{i}, D(\phi)\right), \\
x_{i} \mid T_{i}=t_{i} & \stackrel{\text { ind }}{\sim} N_{1}\left(\mu_{x}+\phi_{x}^{1 / 2} \delta_{x} t_{i}, \phi_{x}\left(1-\delta_{x}^{2}\right)\right), \\
T_{i} & \stackrel{\text { iid }}{\sim} H N(0,1),
\end{aligned}
$$

$i=1, \ldots, n$, todas independentes, em que $H N(0,1)$ denota a distribuição "meionormal" univariada padronizada e $\delta_{x}=\lambda_{x} /\left(1+\lambda_{x}^{2}\right)^{1 / 2}$. Inferência clássica sobre o

vetor de parâmetros $\boldsymbol{\theta}=\left(\boldsymbol{\alpha}^{\top}, \phi^{\top}, \mu_{x}, \phi_{x}, \lambda_{x}\right)^{\top}$ neste tipo de modelo, se baseia na distribuição marginal para a variável resposta $Y_{i}$ sendo derivada no seguinte teorema.

Teorema 3.1. Sob o modelo definido em (3.3)-(3.5), segue que a distribuição marginal de $\mathbf{Y}_{i}$ é dada por

$$
f_{\mathbf{Y}_{i}}\left(\mathrm{y}_{i} \mid \boldsymbol{\theta}\right)=2 \phi_{p}\left(\mathrm{y}_{i} \mid \boldsymbol{\mu}, \boldsymbol{\Sigma}\right) \Phi_{1}\left(\bar{\lambda}_{x}^{\top} \boldsymbol{\Sigma}^{-1 / 2}\left(\mathrm{y}_{i}-\boldsymbol{\mu}\right)\right)
$$

$i=1, \ldots, n, \mathbf{Y}_{i} \stackrel{i i d}{\sim} S N_{p}\left(\boldsymbol{\mu}, \Sigma, \bar{\lambda}_{x}\right), \quad \operatorname{com} \boldsymbol{\mu}=\mathbf{a}+1_{p} \mu_{x}, \quad \Sigma=D(\phi)+\phi_{x} 1_{p} 1_{p}^{\top}, \quad \bar{\lambda}_{x}=$ $\frac{\lambda_{x} \phi_{x} \Sigma^{-1 / 2} 1_{p}}{\sqrt{\phi_{x}+\lambda_{x}^{2} \Lambda_{x}}}$, em que $\Lambda_{x}=\left(\phi_{x}^{-1}+1_{p}^{\top} D^{-1}(\phi) 1_{p}\right)^{-1}$.

Demonstração. A demonstração do Teorema é apresentada no Apêndice B.

\subsubsection{Função Escore}

Esta subseção apresenta a função escore para o vetor de parâmetros $\boldsymbol{\theta}$. De (3.6), e depois, de algumas manipulações algébricas, a função de log-verossimilhança pode ser alternativamente escrita como

$$
\ell(\boldsymbol{\theta})=\sum_{i=1}^{n} \ell_{i}(\boldsymbol{\theta})
$$


em que $\ell_{i}(\theta)=-\frac{1}{2} \log |\Sigma|-\frac{1}{2} d_{i}+\log \left(K_{i}\right)+C$, onde $C$ denota uma constante que não depende do vetor de parâmetro $\boldsymbol{\theta}, d_{i}=\left(\mathrm{y}_{i}-\boldsymbol{\mu}\right)^{\top} \Sigma^{-1}\left(\mathrm{y}_{i}-\boldsymbol{\mu}\right), K_{i}=\Phi\left(A_{x} a_{i}\right)$, com $A_{x}=\frac{\lambda_{x} \Lambda_{x}}{\sqrt{\phi_{x}+\lambda_{x}^{2} \Lambda_{x}}}, a_{i}=\left(\mathrm{y}_{i}-\mu\right)^{\top} D^{-1}(\phi) \mathbf{1}_{p}$ e $\Lambda_{x}=\frac{\phi_{x}}{c} \operatorname{com} c=1+\phi_{x} 1_{p}^{\top} D^{-1}(\phi) \mathbf{1}_{p}$, $i=1, \ldots, n$. A função escore é obtida derivando a função de log-verossimilhança com respeito ao vetor de parâmetros. Temos que

$$
U(\boldsymbol{\theta})=\frac{\partial \ell(\boldsymbol{\theta})}{\partial \boldsymbol{\theta}}=\sum_{i=1}^{n} U_{i}(\boldsymbol{\theta})
$$

onde $U_{i}(\boldsymbol{\theta})=\frac{\partial \ell_{i}(\boldsymbol{\theta})}{\partial \boldsymbol{\theta}}=\left(U_{i}(\boldsymbol{\alpha})^{\top}, U_{i}(\boldsymbol{\phi})^{\top}, U_{i}\left(\mu_{x}\right), U_{i}\left(\phi_{x}\right), U_{i}\left(\lambda_{x}\right)\right)^{\top} \mathrm{e}$

$$
U_{i}(\gamma)=\frac{\partial \ell_{i}(\boldsymbol{\theta})}{\partial \gamma}=-\frac{1}{2} \frac{\partial \log |\Sigma|}{\partial \gamma}-\frac{1}{2} d_{i} \gamma+\frac{\partial \log K_{i}}{\partial \gamma}
$$

onde $d_{i \gamma}=\frac{\partial d_{i}}{\partial \gamma}, \frac{\partial \log K_{i}}{\partial \gamma}=W_{\Phi_{1}}\left(A_{x} a_{i}\right)\left\{A_{x} \frac{\partial a_{i}}{\partial \gamma}+a_{i} \frac{\partial A_{x}}{\partial \gamma}\right\}, \operatorname{com} W_{\Phi_{1}}(u)=\phi_{1}(u) / \Phi_{1}(u)$, $u \in \mathbb{R}, \gamma=\mu_{x}, \alpha, \phi_{x}, \phi, \lambda_{x}, i=1, \ldots, n$. As expressões das derivadas descritas acima são apresentadas no Apêndice D.1.

\subsubsection{Matriz de informação de Fisher observada}

Nesta subseção apresentamos a matriz de informação de Fisher observada para o vetor de parâmetros $\theta$ e é denotada por

$$
\mathbf{L}=\frac{\partial^{2} \ell(\boldsymbol{\theta})}{\partial \boldsymbol{\theta} \partial \boldsymbol{\theta}^{\top}}=\sum_{i=1}^{n} \frac{\partial^{2} \ell_{i}(\boldsymbol{\theta})}{\partial \boldsymbol{\theta} \partial \boldsymbol{\theta}^{\top}} .
$$

De (3.8) podemos escrever cada elemento da matriz de informação de Fisher observada como

$$
\mathbf{J}_{i}=-\mathbf{L}_{i}(\boldsymbol{\theta})=-\frac{\partial^{2} \ell_{i}(\boldsymbol{\theta})}{\partial \gamma \partial \tau^{\top}}
$$


em que $\frac{\partial^{2} \ell_{i}}{\partial \gamma \partial \tau^{\top}}=-\frac{1}{2} \frac{\partial^{2} \log |\Sigma|}{\partial \gamma \partial \tau^{\top}}-\frac{1}{2} d_{i \gamma \tau^{\top}}+\frac{\partial^{2} \log K_{i}}{\partial \gamma \partial \tau^{\top}}$, com

$$
\begin{aligned}
\frac{\partial^{2} \log K_{i}}{\partial \gamma \partial \tau^{\top}}= & W_{\Phi_{1}}\left(A_{x} a_{i}\right)\left\{\frac{\partial A_{x}}{\partial \gamma} \frac{\partial a_{i}}{\partial \tau^{\top}}+A_{x} \frac{\partial^{2} a_{i}}{\partial \gamma \partial \tau^{\top}}+\frac{\partial a_{i}}{\partial \gamma} \frac{\partial A_{x}}{\partial \tau^{\top}}+a_{i} \frac{\partial^{2} A_{x}}{\partial \gamma \partial \tau^{\top}}\right\} \\
& +\Delta_{\Phi_{1}}\left(A_{x} a_{i}\right)\left\{A_{x} \frac{\partial a_{i}}{\partial \gamma}+a_{i} \frac{\partial A_{x}}{\partial \gamma}\right\}\left\{A_{x} \frac{\partial a_{i}}{\partial \tau^{\top}}+a_{i} \frac{\partial A_{x}}{\partial \tau^{\top}}\right\}
\end{aligned}
$$

em que $\Delta_{\Phi_{1}}(u)=W_{\Phi_{1}}^{\prime}(u)=-W_{\Phi_{1}}(u)\left(u+W_{\Phi_{1}}(u)\right), u \in \mathbb{R}, d_{i \gamma \tau^{\top}}=\frac{\partial^{2} d_{i}}{\partial \gamma \partial \tau^{\top}}$ $\gamma, \tau=\mu_{x}, \alpha, \phi_{x}, \phi, \lambda_{x}$. As derivadas segundas são apresentadas no Apêndice E.1. Regiões de confiança assintóticas e testes de hipóteses baseados nos estimadores de máxima verossimilhança (EMV's) podem ser obtidos usando a matriz $\mathbf{J}=-\mathbf{L}$, que denota a matriz de informação de Fisher observada nos parâmetros do modelo MGSN. Assim, regiões de confiança assintóticas levando em conta que o $\operatorname{EMV}(\widehat{\boldsymbol{\theta}})$ tem aproximadamente uma distribuição $N_{2 p+1}\left(\boldsymbol{\theta}, \mathbf{J}^{-1}\right)$ (Sen e Singer, 1993) e testes de hipóteses para o parâmetro $\theta$ podem ser obtidos. Como $\mathbf{J}$ é desconhecida, substituímos pelo $\operatorname{EMV}(\widehat{\mathbf{J}})$ que é a matriz $\mathbf{J}$ avaliada nos EMV's $\hat{\boldsymbol{\theta}}$.

\subsubsection{Estimação de máxima verossimilhança via algoritmo EM}

Nesta seção discutimos o EMV dos parâmetros do vetor $\boldsymbol{\theta}$ para o MGSN. Considerando $\mathbf{y}=\left(\mathbf{y}_{1}^{\top}, \ldots, \mathbf{y}_{n}^{\top}\right)^{\top}, \mathbf{x}=\left(x_{1}, \ldots, x_{n}\right)^{\top}$ e $\mathbf{t}=\left(t_{1}, \ldots, t_{n}\right)^{\top}$, implementamos $p$ algoritmos EM para o MGSN utilizando o aumento duplo, onde $(\mathbf{x}, \mathbf{t})$ são conhecidos como dados perdidos ou latentes. Assim, sob a representação hierárquica (3.3)-(3.5), com $\nu^{2}=\phi_{x}\left(1-\delta_{x}^{2}\right)$ e $\varsigma=\phi_{x}^{1 / 2} \delta_{x}$, segue que a função de log-verossimilhança completa 
associada com $(\mathrm{y}, \mathrm{x}, \mathrm{t})$ é dada por

$$
\begin{aligned}
\ell_{c}(\boldsymbol{\theta} \mid \mathbf{y}, \mathbf{x}, \mathbf{t})= & -\frac{n}{2} \log (|D(\phi)|)-\frac{1}{2} \sum_{i=1}^{n}\left(\mathbf{y}_{i}-\mathbf{a}-\mathbf{1}_{p} x_{i}\right)^{\top} D^{-1}(\phi)\left(\mathbf{y}_{i}-\mathbf{a}-\mathbf{1}_{p} x_{i}\right) \\
& -\frac{n}{2} \log \left(\nu^{2}\right)-\frac{1}{2 \nu^{2}} \sum_{i=1}^{n}\left(x_{i}-\mu_{x}-\varsigma t_{i}\right)^{2}+C,
\end{aligned}
$$

onde $C$ é uma constante que independe do vetor de parâmetros $\theta$. Assim, o algoritmo EM resulta nos seguintes passos:

Passo-E: Dado $\boldsymbol{\theta}=\widehat{\boldsymbol{\theta}}$, calculamos $\widehat{t}_{i}, \widehat{t}^{2}, \widehat{x}_{i}, \widehat{x}_{i}^{2}$ e $\widehat{t x}_{i}$ para $i=1, \ldots, n$, conforme abaixo

Seja $\widehat{x}_{i}=E\left[x_{i} \mid \boldsymbol{\theta}=\widehat{\boldsymbol{\theta}}, \mathbf{y}_{i}\right], \widehat{x_{i}^{2}}=E\left[x_{i}^{2} \mid \boldsymbol{\theta}=\widehat{\boldsymbol{\theta}}, \mathbf{y}_{i}\right], \widehat{t_{i}}=E\left[T_{i} \mid \boldsymbol{\theta}=\widehat{\boldsymbol{\theta}}, \mathbf{y}_{i}\right], \widehat{t_{i}^{2}}=E\left[T_{i}^{2} \mid \boldsymbol{\theta}=\right.$

$\left.\widehat{\boldsymbol{\theta}}, \mathrm{y}_{i}\right]$ e $\widehat{t x_{i}}=E\left[T_{i} x_{i} \mid \boldsymbol{\theta}=\widehat{\boldsymbol{\theta}}, \mathrm{y}_{i}\right]$, quxe são obtidas usando esperança condicional dupla e os momentos da distribuição normal truncada, a demonstração destas expressões podenser verificadås no Apêndice $\mathrm{C}$, levando a

$$
\begin{aligned}
\widehat{t_{i}} & =\widehat{\mu}_{T i}+W_{\Phi_{1}}\left(\frac{\widehat{\mu}_{T_{i}}}{\widehat{M}_{T}}\right) \widehat{M}_{T} \\
\widehat{t_{i}^{2}} & =\widehat{\mu}_{T_{i}}^{2}+\widehat{M}_{T}^{2}+W_{\Phi_{1}}\left(\frac{\widehat{\mu}_{T_{i}}}{\widehat{M}_{T}}\right) \widehat{M}_{T} \widehat{\mu}_{T_{i}}, \\
\widehat{x}_{i} & =\widehat{r}_{i}+\widehat{s} \widehat{t}_{i}, \\
\widehat{x_{i}^{2}} & =\widehat{T}_{x}^{2}+\widehat{r}_{i}^{2}+2 \widehat{r}_{i} \widehat{s} \widehat{t}_{i}+\widehat{s}^{2} \widehat{t}_{i}^{2}
\end{aligned}
$$

$\mathrm{e}$

$$
\widehat{t x}_{i}=\widehat{r}_{i} \widehat{t}_{i}+\widehat{s} \widehat{t}_{i}^{2}
$$

em que $\widehat{M}_{T}^{2}=\left[1+\widehat{\varsigma}^{2} \mathbf{1}_{p}^{\top}\left(D(\widehat{\phi})+\widehat{\nu}^{2} \mathbf{1}_{p} \mathbf{1}_{p}^{\top}\right)^{-1} \mathbf{1}_{p}\right]^{-1}, \widehat{\mu}_{T_{i}}=\widehat{\varsigma} \widehat{M}_{T}^{2} \mathbf{1}_{p}^{\top}\left(D(\widehat{\phi})+\widehat{\nu}^{2} \mathbf{1}_{p} \mathbf{1}_{p}^{\top}\right)^{-1}\left(\mathrm{y}_{i}-\right.$ $\left.\mathbf{a}-\mathbf{1}_{p} \widehat{\mu}_{x}\right), \widehat{T}_{x}^{2}=\widehat{\nu}^{2}\left[1+\widehat{\nu}^{2} \mathbf{1}_{p}^{\top} D^{-1}(\widehat{\phi}) \mathbf{1}_{p}\right]^{-1}, \widehat{r}_{i}=\widehat{\mu}_{x}+\widehat{T}_{x}^{2} 1_{p}^{\top} D^{-1}(\widehat{\phi})\left(\mathbf{y}_{i}-\mathbf{a}-\mathbf{1}_{p} \widehat{\mu}_{x}\right)$ e $\widehat{s}=$ $\widehat{\varsigma}\left(1-\widehat{T}_{x}^{2} 1_{p}^{\top} D^{-1}(\widehat{\phi}) 1_{p}\right)$. Conseqüentemente, a esperança condicional correspondente a 
este passo tem a forma

$$
\begin{aligned}
& Q(\boldsymbol{\theta}, \widehat{\theta})=E_{\mathrm{x}, \mathrm{t}}\left[\ell_{c}(\boldsymbol{\theta} \mid \mathrm{y}, \mathbf{x}, \mathbf{t}) \mid \mathbf{y}, \widehat{\theta}\right]=-\frac{n}{2} \log (|D(\phi)|) \\
& -\frac{1}{2} \sum_{i=1}^{n}\left(\mathbf{y}_{i}-\mathbf{a}-1_{p} \widehat{x}_{i}\right)^{\top} D^{-1}(\phi)\left(\mathbf{y}_{i}-\mathbf{a}-1_{p} \widehat{x}_{i}\right)-\frac{1}{2} \mathbf{1}_{p}^{\top} D^{-1}(\phi) \mathbf{1}_{p} \sum_{i=1}^{n}\left(\widehat{x_{i}^{2}}-\widehat{x}_{i}^{2}\right) \\
& -\frac{n}{2} \log \left(\nu^{2}\right)-\frac{1}{2 \nu^{2}} \sum_{i=1}^{n}\left(\widehat{x_{i}^{2}}+\mu_{x}^{2}+\varsigma^{2} \widehat{t}_{i}^{2}-2 \widehat{x}_{i} \mu_{x}-2 \varsigma \widehat{x t_{i}}+2 \varsigma \mu_{x} \widehat{t_{i}}\right)+C
\end{aligned}
$$

Passo-M: Dado $\widehat{\boldsymbol{\theta}}$, maximizar $E\left[\ell_{c}(\boldsymbol{\theta} \mid \mathbf{y}, \mathbf{x}, \mathbf{t}) \mid \mathbf{y}, \widehat{\boldsymbol{\theta}}\right]$ em (3.9) em relação a $\boldsymbol{\theta}$. Como solução obtemos

$$
\begin{aligned}
\widehat{\alpha}_{j} & =\bar{y}_{j}-\overline{\widehat{x}}, \\
\widehat{\phi}_{1} & =\frac{1}{n} \sum_{i=1}^{n}\left(y_{i 1}^{2}-2 \widehat{x}_{i} y_{i 1}+\widehat{x_{i}^{2}}\right), \\
\widehat{\phi}_{j} & =\frac{1}{n} \sum_{i=1}^{n}\left(y_{i j}^{2}+\alpha_{j}^{2}+\widehat{x}^{2}{ }_{i}-2 \alpha_{j} y_{i j}-2 y_{i j} \widehat{x}_{i}+2 \alpha_{j} \widehat{x}_{i}\right), \\
\widehat{\mu}_{x} & =\frac{1}{n} \sum_{i=1}^{n}\left(\widehat{x}_{i}-\widehat{\iota t}_{i}\right), \\
\widehat{\nu}^{2} & =\frac{1}{n} \sum_{i=1}^{n}\left(\widehat{x_{i}^{2}}+\mu_{x}^{2}+\varsigma^{2} \widehat{t_{i}^{2}}-2 \mu_{x} \widehat{x}_{i}-2 \varsigma \widehat{t x_{i}}+2 \varsigma \mu_{x} \widehat{t}_{i}\right), \mathrm{e} \\
\widehat{\varsigma} & =\frac{\sum_{i=1}^{n}\left(\widehat{t x_{i}}-\mu_{x} \widehat{t}_{i}\right)}{\sum_{i=1}^{n} \widehat{t}_{i}^{2}},
\end{aligned}
$$

em que $\bar{y}_{j}=\frac{1}{n} \sum_{i=1}^{n} y_{i j}, \overline{\widehat{x}}=\frac{1}{n} \sum_{i=1}^{n} \widehat{x}_{i}$ e $j=2, \ldots, p$. Os parâmetros de forma e escala da variável latente $x$ podem ser estimados usando as relações $\lambda_{x}=\varsigma / \nu$ e $\phi_{x}=\varsigma^{2}+\nu^{2}$. Os valores iniciais dos parâmetros (exceto $\lambda_{x}$ ) são freqüentemente escolhidos como sendo as estimativas do modelo de Grubbs Normal, onde o valor inicial para o parâmetro de assimétria é igual a zero. Note que sob $\lambda_{x}=0$ (ou $\varsigma=0$ ), o passo M reduz-se às equações obtida em Vilca et al. (2002). Na literatura recomenda-se utilizar o algoritmo EM várias vezes com diferentes valores inciais. 


\subsection{Esquemas de perturbação para o MGSN}

Nesta seção derivamos a matriz $\Delta$ de (2.23) para diferentes esquemas de perturbação.

\subsubsection{Perturbação da ponderação de casos}

Note que a função de log-verossimilhança para o modelo (3.1)-(3.2) é dada por (3.7) onde $\ell_{i}(\boldsymbol{\theta})$ é a contribuição da $i$-ésima observação (igualmente ponderado) para a verossimilhança, $i=1, \ldots, n$. A função de log-verossimilhança perturbada incorpora diferentes pesos para diferentes observações, sendo definida por

$$
\ell(\boldsymbol{\theta} \mid \omega)=\sum_{i=1}^{n} \omega_{i} \ell_{i}(\boldsymbol{\theta}),
$$

em que, $\boldsymbol{\theta}=\left(\boldsymbol{\alpha}^{\top}, \phi^{\top}, \mu_{x}, \phi_{x}, \lambda_{x}\right)^{\top}$ e $\boldsymbol{\omega}=\left(\omega_{1}, \ldots, \omega_{n}\right)^{\top}$ são os vetores de parâmetros e pesos, respectivamente. Neste caso, $\boldsymbol{\omega}_{0}=\mathbf{1}_{n}=(1, \ldots, 1)^{\top}$ significa ausência de perturbação, isto é, $l\left(\theta \mid \omega_{0}\right)=l(\theta)$. Assim, o esquema de perturbação pretende avaliar se a contribuição das observações com diferentes pesos afeta o estimador de máxima verossimilhança de $\boldsymbol{\theta}$. Provavelmente, este método seja o mais usado para avaliar a influência de pequenas perturbações no modelo. Portanto, usando (3.10) e depois de algumas manipulações algébricas, a matriz $\Delta$ em (2.23) é dada por

$$
\Delta=\left(\Delta_{1}, \ldots, \Delta_{n}\right)
$$

em que $\Delta_{i}=\frac{\partial \ell_{i}(\boldsymbol{\theta})}{\partial \boldsymbol{\theta}}, i=1, \ldots, n$, com elementos apresentados no Apêndice D.1 (veja também Seção 3.2.1). 


\subsubsection{Perturbação das medições de um dos instrumentos}

Nesta seção as medidas tomadas como obtidas quando um instrumento são modificadas segundo esquemas de perturbação aditivos e multiplicativos. Supondo que as medidas do instrumento $m \in\{=1, \ldots, p\}$ são escolhidas para serem perturbadas, o modelo perturbado é dado por

$$
Y_{m i}(\omega)=\mathbf{a}+\mathbf{1}_{p} x_{i}+\epsilon_{i}
$$

com

$$
\mathbf{Y}_{m i}\left(\omega_{i}\right)=\left\{\begin{array}{c}
\mathbf{Y}_{i}+\omega_{i} \mathbf{e}_{m}, \text { perturbação aditiva; } \\
\mathbf{Y}_{i} \boxminus \mathbf{1}_{m}\left(\omega_{i}\right), \text { perturbação multiplicativa }
\end{array}\right.
$$

em que $\mathbf{e}_{m}$ é um vetor de dimensão $p$ com um na $m$-ésima posição, e zero nas demais e $\mathbf{1}_{m}\left(\omega_{i}\right)$ denota um vetor de uns de dimensão $p$ com o $m$-ésimo componente substituído por $\omega_{i}$ e " $\square$ " denota o produto de Hadamard.

Ausência de perturbação segue tomando $\omega_{o}=0$ no caso aditivo e $\omega_{o}=1$ para o caso multiplicativo. A função de log-verossimilhança perturbada segue de (3.7) com $\mathrm{y}_{i}$ substituído por $\mathrm{y}_{m i}\left(\omega_{i}\right), i=1, \ldots, n$, ou seja,

$$
\ell(\boldsymbol{\theta} \mid \boldsymbol{\omega})=\sum_{i=1}^{n} \ell_{i}\left(\boldsymbol{\theta} \mid \omega_{i}\right)
$$

em que $\ell_{i}\left(\boldsymbol{\theta} \mid \omega_{i}\right)=-\frac{1}{2} \log |\Sigma|-\frac{1}{2} d_{m i}\left(\omega_{i}\right)+\log \left(K_{m i}\left(\omega_{i}\right)\right)+C \operatorname{com} d_{m i}\left(\omega_{i}\right)=\left(\mathbf{y}_{m i}\left(\omega_{i}\right)-\right.$ $\boldsymbol{\mu})^{\top} \Sigma^{-1}\left(\mathbf{y}_{m i}\left(\omega_{i}\right)-\boldsymbol{\mu}\right), K_{m i}=\Phi\left(A_{x} a_{m i}\left(\omega_{i}\right)\right)$ e $a_{m i}\left(\omega_{i}\right)=\left(\mathbf{y}_{m i}\left(\omega_{i}\right)-\boldsymbol{\mu}\right)^{\top} D^{-1}(\phi) b$.

Derivando $\ell(\boldsymbol{\theta} \mid \boldsymbol{\omega})$ com respeito a $\boldsymbol{\omega}$ e $\theta$, temos

$$
\Delta=\left(\Delta_{1}^{m}, \ldots, \Delta_{n}^{m}\right)
$$


em que

$$
\begin{aligned}
\Delta_{i \boldsymbol{\theta}}^{m}= & \frac{\partial T_{m i}\left(\omega_{i}\right)}{\partial \boldsymbol{\theta}}+W_{\Phi_{1}}\left(A_{x} a_{m i}\left(\omega_{i}\right)\right)\left\{\frac{\partial A_{x}}{\partial \boldsymbol{\theta}} S_{m i}+A_{x} \frac{\partial S_{m i}\left(\omega_{i}\right)}{\partial \boldsymbol{\theta}}\right\} \\
& +A_{x} W_{\Phi_{1}}^{\prime}\left(A_{x} a_{m i}\left(\omega_{i}\right)\right) S_{m i}\left\{A_{x} \frac{\partial a_{m}\left(\omega_{i}\right)}{\partial \boldsymbol{\theta}}+a_{m i}\left(\omega_{i}\right) \frac{\partial A_{x}}{\partial \boldsymbol{\theta}}\right\}
\end{aligned}
$$

$\operatorname{com} T_{m i}\left(\omega_{i}\right)=-\left(\mathbf{y}_{m i}\left(\omega_{i}\right)-\boldsymbol{\mu}\right)^{\top} \Sigma^{-1} \frac{\partial \mathbf{y}_{m i}\left(\omega_{i}\right)}{\partial \omega_{i}}, \quad S_{m i}\left(\omega_{i}\right)=\mathrm{b}^{\top} D^{-1}(\phi) \frac{\partial \mathbf{y}_{m i}\left(\omega_{i}\right)}{\partial \omega_{i}} \mathrm{e}$ $\frac{\partial a_{m i}\left(\omega_{i}\right)}{\partial \boldsymbol{\theta}}$ para o caso não perturbado, substituímos $\mathbf{y}_{i}$ por $\mathbf{y}_{m i}\left(\omega_{i}\right)$ (ver Apêndice F.1). Note que $\frac{\partial \mathbf{y}_{m i}\left(\omega_{i}\right)}{\partial \omega_{i}}=\mathbf{e}_{m}$ para o caso aditivo e $\frac{\partial \mathbf{y}_{m i}\left(\omega_{i}\right)}{\partial \omega_{i}}=1_{m}\left(y_{i m}\right)$ para o caso multiplicativo, $i=1, \ldots, n$.

\subsubsection{Perturbação do viés multiplicativo}

Consideramos o seguinte modelo perturbado:

$$
\mathrm{Y}_{i}=\mathbf{a}+\mathrm{b}_{\omega} x_{i}+\epsilon_{i}
$$

em que $\mathbf{a}=\left(0, \boldsymbol{\alpha}^{\top}\right)^{\top}$ e $\mathbf{b}_{\omega}=\left(1, \boldsymbol{\omega}^{\top}\right)^{\top}$, com $\boldsymbol{\omega}=\left(\omega_{2}, \ldots, \omega_{p}\right)^{\top}$. O modelo sem perturbação segue tomando $\omega_{o}=1_{p-1}$ ou $\mathrm{b}_{\omega_{o}}=1_{p}$. Procedendo similarmente como em (3.6), pode-se mostrar que sob este esquema de perturbação, $\mathbf{Y}_{i} \stackrel{i i d}{\sim} S N_{p}\left(\boldsymbol{\mu}_{w}, \Sigma_{w}, \bar{\lambda}_{w}\right)$, $i=1, \ldots, n$, onde $\mu_{\omega}=\mathbf{a}+\mathbf{b}_{\omega} \mu_{x}, \Sigma_{\omega}=D(\phi)+\phi_{x} \mathbf{b}_{\omega} \mathbf{b}_{\omega}^{\top}$ e $\bar{\lambda}_{\omega}=\frac{\lambda_{x} \phi_{x} \Sigma_{\omega}^{-1 / 2} \mathbf{b}_{\omega}}{\sqrt{\phi_{x}+\lambda_{x}^{2} \Lambda_{x \omega}}}$, com $\Lambda_{x \omega}=\left(\phi_{x}^{-1}+\mathbf{b}_{\omega}^{\top} D^{-1}(\phi) \mathbf{b}_{\omega}\right)^{-1}$.

Note que, sob este esquema de perturbação, obtemos o modelo originalmente proposto em Barnett (1969), onde $\mathbf{b}_{\omega}$ é associado com o viés multiplicativo dos dispositivos medidores. Este modelo estende o modelo de Grubbs por incluir vieses aditivos e multiplicativos. A função de log-verossimilhança para o modelo perturbado é dada por

$$
\ell(\theta \mid \omega)=\sum_{i=1}^{n} \ell_{i}(\theta \mid \omega)
$$


em que $\ell_{i}(\boldsymbol{\theta} \mid \boldsymbol{\omega})=\log (2)-(p / 2) \log (2 \pi)-\frac{1}{2} \log \left|\Sigma_{\omega}\right|-\frac{1}{2} d_{i \omega}+\log \left(K_{i \omega}\right)$, com $d_{i \omega}=$ $\left(\mathrm{Y}_{i}-\boldsymbol{\mu}_{\omega}\right)^{\top} \Sigma_{\omega}^{-1}\left(\mathrm{Y}_{i}-\boldsymbol{\mu}_{\omega}\right)$ e $K_{i \omega}=\Phi_{1}\left(\bar{\lambda}_{x \omega}^{\top} \Sigma_{\omega}^{-1 / 2}\left(\mathrm{y}_{i}-\mu_{\omega}\right)\right)=\Phi_{1}\left(A_{x \omega} a_{i \omega}\right), \operatorname{com} A_{x \omega}=$ $\lambda_{x} \Lambda_{x \omega} /\left(\phi_{x}+\lambda_{x}^{2} \Lambda_{x \omega}\right)^{1 / 2}$ e $a_{i \omega}=X_{i \omega}^{\top} D^{-1}(\phi) \mathbf{b}_{\omega}, \mathbf{X}_{i \omega}=\mathbf{Y}_{i}-\mathbf{a}-\mathbf{b}_{\omega} \mu_{x}$

A matriz $\Delta$ é dada por

$$
\Delta=\sum_{i=1}^{n} \frac{\partial^{2} l_{i}(\theta \mid \omega)}{\partial \theta \partial \omega^{\top}}
$$

em que os elementos de $\frac{\partial^{2} l_{i}(\boldsymbol{\theta} / \boldsymbol{\omega})}{\partial \boldsymbol{\theta} \partial \boldsymbol{\omega}^{\top}}$ são apresentados no Apêndice E.1. A matriz $\Delta$ definida acima deve ser avaliada nas estimativas de máxima verossimilhança e em $\omega=1_{p-1}$.

\subsection{Aplicações}

Nesta seção apresentamos duas aplicações da metodologia discutida neste capítulo. O primeiro conjunto de dados foi previamente analisado em Grubbs (1948) e o segundo analisado em Barnett (1969).

\subsubsection{Dados de Grubbs}

Consideramos inicialmente o conjunto de dados estudado em Grubbs (1948): Os dados são relacionados ao tempo de queima de projéteis para fuzis registrados por três diferentes observadores (ver Tabela 3.1). Este conjunto de dados também foi analisado por Jaech (1985) e por Lachos et al. (2006), ambos sob a suposição de normalidade. Comparamos os modelos MGN e MGSN para este conjunto de dados. As estimativas dos parâmetros são apresentadas na Tabela 3.2. Note que os critérios de informação AIC, BIC e HQ (valores mostrados na parte inferior da Tabela 3.2) 
favorecem o MGSN, contra a suposição de normalidade. Uma conclução mais enfática neste sentido é conseguida considerando-se um teste paramétrico para normalidade, onde a hipótese nula é $\lambda_{x}=0$, com o teste da razão de verossimilhanças para o qual o nível crítico associado a 5\% é 3.84, com 1 grau de liberdade. Esta conclusão é também reforçada pelo gráfico PP tipo Healy (Azzalini e Capitanio, 1999) mostrado na Figura 3.1. Para a construção deste gráfico, consideramos

$$
d_{i}=\left(\mathbf{y}_{i}-\boldsymbol{\mu}\right)^{\top} \Sigma^{-1}\left(\mathbf{y}_{i}-\boldsymbol{\mu}\right) \stackrel{i i d}{\sim} \chi_{p}^{2}(i=1, \ldots, n),
$$

(Arellano-Valle e Genton, 2005) que são amostradas de uma distribuição $\chi_{p}^{2}$. $\mathrm{Na}$ prática, as estimativas podem substituir os valores dos parâmetros em (3.11). Desta forma, os $d_{i}$ podem ser aleatorizados e graficados contra os quanties da distribuição $\chi_{p}^{2}$. De forma equivalente, podemos trabalhar no lugar dos quanties com as probabilidades $p(i)$ da função distribuição $\chi_{p}^{2}$ avaliado em $\left.d_{(} i\right)$ contra seus valores nominais $i /(n+1), i=1, \ldots, n$, quanto mais próximos os pontos estiverem da bissetriz do primeiro quadrante mais próximos os dados observados estão da distribuição considerada (ver programa na Apêndice G.2).

Para este conjunto de dados o estudo de influência local baseia-se em perturbação de casos com interesse em $\boldsymbol{\theta}$. Os resultados são mostrados na Figura 3.2. As observações 2 e 4 parecem ser bastante influentes, como visto na Figura 3.2 (a). As observações 4 e 26 parecem ser influentes, conforme a influência local total $C_{i}$ como mostra a Figura 3.2 (b). Além disso, a partir do afastamento da verossimilhança e pela distância de Cook apresentados nas Figuras 3.2 (c) e (d), respectivamente, nota-se que as observações 17 e 26 são possivelmente globalmente influentes.

Agora examinamos os efeitos de perturbação das medidas tomadas pelos três observadores. Os valores de $C_{d \max }$ para a perturbação aditiva são $17603(A), 5970(B)$ e $13594(C)$, enquanto que para a perturbação multiplicativa os valores de $C_{d m a x}$ são 
$16541100(A), 575780(B)$ e $1282700(C)$. A Figura 3.3 ilustra o gráfico de índice para este esquema de perturbação. Neste caso, o observador $A$ é escolhido porque apresenta os maiores valores $C_{d m a x}$, ou seja, em ambos métodos de perturbação tanto aditivo como multiplicativo, o observador $A$ é quem se destaca em relação aos outros observadores.

Com a finalidade de comparar com o modelo normal, a Figura 3.4 mostra alguns resultados sob MGN (ver também Lachos et al., 2006). Note que as conclusões são diferentes, porque sob o modelo normal-assimétrico as observações 4, 17 e 26 são as mais influentes, enquanto que, sob o modelo normal, as observações mais influentes são 4 e 17. Note também que a observação 26 apresenta a menor média entre todas as observações, como mostrado na Tabela 3.1. 
Tabela 3.1: Conjunto de dados de Grubbs. Tempo de queima de projéteis em segundos, medido por cada um dos três observadores.

\begin{tabular}{c|ccc|cc}
\hline & observador & observador & observador & Tempo & Desvio \\
$N^{\circ}$ & $\mathrm{A}$ & $\mathrm{B}$ & $\mathrm{C}$ & Médio & Padrão \\
\hline \hline 1 & 10.10 & 10.07 & 10.07 & 10.080 & 0.0173 \\
2 & 9.98 & 9.90 & 9.90 & 9.9267 & 0.0462 \\
3 & 9.89 & 9.85 & 9.86 & 9.8667 & 0.0208 \\
4 & 9.79 & 9.71 & 9.70 & 9.7333 & 0.0493 \\
5 & 9.67 & 9.65 & 9.65 & 9.6567 & 0.0115 \\
6 & 9.89 & 9.83 & 9.83 & 9.8500 & 0.0346 \\
7 & 9.82 & 9.75 & 9.79 & 9.7867 & 0.0351 \\
8 & 9.59 & 9.56 & 9.59 & 9.5800 & 0.0173 \\
9 & 9.76 & 9.68 & 9.72 & 9.7200 & 0.0400 \\
10 & 9.93 & 9.89 & 9.92 & 9.9133 & 0.0208 \\
11 & 9.62 & 9.61 & 9.64 & 9.6233 & 0.0153 \\
12 & 10.24 & 10.23 & 10.24 & 10.237 & 0.0058 \\
13 & 9.84 & 9.83 & 9.86 & 9.8433 & 0.0153 \\
14 & 9.62 & 9.58 & 9.63 & 9.6100 & 0.0265 \\
15 & 9.60 & 9.600 & 9.65 & 9.6167 & 0.0289 \\
16 & 9.74 & 9.73 & 9.74 & 9.7367 & 0.0058 \\
17 & 10.32 & 10.32 & 10.34 & 10.327 & 0.0115 \\
18 & 9.86 & 9.86 & 9.86 & 9.8600 & 0.0000 \\
19 & 9.65 & 9.64 & 9.65 & 9.6467 & 0.0058 \\
20 & 9.50 & 9.49 & 9.50 & 9.4967 & 0.0058 \\
21 & 9.56 & 9.56 & 9.55 & 9.5567 & 0.0058 \\
22 & 9.54 & 9.53 & 9.54 & 9.5367 & 0.0058 \\
23 & 9.89 & 9.89 & 9.88 & 9.8867 & 0.0058 \\
24 & 9.53 & 9.52 & 9.51 & 9.5200 & 0.0100 \\
25 & 9.52 & 9.52 & 9.53 & 9.5233 & 0.0058 \\
\hline & 9.44 & 9.43 & 9.45 & 9.4400 & 0.0100 \\
27 & 9.67 & 9.67 & 9.67 & 9.6700 & 0.0000 \\
28 & 9.77 & 9.76 & 9.78 & 9.7700 & 0.0100 \\
& 9.86 & 9.84 & 9.86 & 9.8533 & 0.0115 \\
\hline & & & & & \\
\hline
\end{tabular}


Tabela 3.2: Resultados do ajuste do modelo de Grubbs normal e normal-assimétrico (MGN e MGSN) para o conjunto de dados de Grubbs. EP são as estimativas dos erros padrão assintóticos baseados na matriz de informação de Fisher observada apresentada no Apêndice E.1.

\begin{tabular}{ccccc}
\hline \hline & \multicolumn{2}{c}{ MGN } & \multicolumn{2}{c}{ MGSN } \\
Parâmetros & Estimativas & EP & Estimativas & EP \\
\hline$\mu_{x}$ & 9.7414 & 0.0387 & 9.4733 & 0.0353 \\
$\alpha_{2}$ & 0.0238 & 0.0048 & 0.0238 & 0.0048 \\
$\alpha_{3}$ & 0.0141 & 0.0032 & 0.0141 & 0.0032 \\
$\phi_{x}$ & 0.0434 & 0.0114 & 0.1151 & 0.0355 \\
$\phi_{1}$ & 0.0001 & 0.0001 & 0.0001 & 0.0001 \\
$\phi_{2}$ & 0.0006 & 0.0002 & 0.0006 & 0.0002 \\
$\phi_{3}$ & 0.0002 & 0.0001 & 0.0002 & 0.0001 \\
$\lambda_{x}$ & - & - & 7.4673 & 5.5488 \\
& & & & \\
\hline BIC & -125.4635 & -128.5686 \\
HQ & -117.4635 & -119.5686 \\
AIC & -107.5999 & -108.4720 \\
\hline log-verossinmilhança & -113.4917 & -1003 \\
\hline \hline
\end{tabular}




\subsubsection{Dados de Barnett}

Nesta segunda aplicação analisamos o conjunto de dados de Barnett (1969). Dois instrumentos (padrão e novo) usados para medir a capacidade vital do pulmão humano, foram comparados em um grupo comum de 72 pacientes. Consideramos as medidas divididas por 100 a fim de conseguir estabilidade numérica. Este conjunto de dados tem sido extensivamente utilizado na literatura no contexto simétrico tais como modelos normais e t. Com este conjunto de dados, estudos de influência local na literatura têm levado a resultados similares, como mostrado em Lachos et al. (2006) e não apresentados aqui. Por esta razão, focalizamos o estudo na perturbação do viés multiplicativo.

Resultados das estimativas dos parâmetros para o ajuste dos MGN e MGSN são fornecidos na Tabela 3.4. As duas últimas colunas (MBarSN) são as estimativas dos parâmetros do modelo de calibração comparativa sob o modelo de Barnett normalassimétrico, apresentado em Lachos et al. (2006) e são apresentados aqui com o propósito de comparação. Os valores de AIC, BIC e HQ mostrados na parte inferior da Tabela 3.4 favorecem o MGSN em relação ao MGN. O gráfico PP apresentado na Figura 3.5 mostra uma visível melhora na adequação do ajuste sob o MGSN.

Na Figura 3.6 apresentamos gráficos de influência local para a perturbação do viés multiplicativo (Seção 3.3.3). Note que os valores de $d \max$ e $C_{i}$ são muito diferentes para cada um dos instrumentos. Assim, podemos concluir que a suposição de vieses multiplicativos iguais não é plausível neste caso. De fato, o máximo da logverossimilhança para a MGSN é -792.8011 e para MGN é -797.6962. Isto indica que o MGSN ajusta os dados significativamente melhor do que o MGSN. 
Tabela 3.3: Resultados do ajuste do modelo de Grubbs normal e normal-assimétrico (MGN e MGSN) para o conjunto de dados de Grubbs. EP são as estimativas dos erros padrão assintóticos baseados na matriz de informação de Fisher observada apresentada no Apêndice E.1.

\begin{tabular}{ccccc}
\hline \hline & \multicolumn{2}{c}{ MGN } & \multicolumn{2}{c}{ MGSN } \\
Parâmetros & Estimativas & EP & Estimativas & EP \\
\hline$\mu_{x}$ & 9.7414 & 0.0387 & 9.4733 & 0.0353 \\
$\alpha_{2}$ & 0.0238 & 0.0048 & 0.0238 & 0.0048 \\
$\alpha_{3}$ & 0.0141 & 0.0032 & 0.0141 & 0.0032 \\
$\phi_{x}$ & 0.0434 & 0.0114 & 0.1151 & 0.0355 \\
$\phi_{1}$ & 0.0001 & 0.0001 & 0.0001 & 0.0001 \\
$\phi_{2}$ & 0.0006 & 0.0002 & 0.0006 & 0.0002 \\
$\phi_{3}$ & 0.0002 & 0.0001 & 0.0002 & 0.0001 \\
$\lambda_{x}$ & - & - & 7.4673 & 5.5488 \\
& & & & \\
\hline - log-verossinmilhança & -125.4635 & -128.5686 \\
AIC & -117.4635 & -119.5686 \\
BIC & -107.5999 & -108.4720 \\
HQ & -113.4917 & -1003 \\
\hline \hline
\end{tabular}


Figura 3.1: Dados de Grubbs. Gráfico PP de Healy para (a) modelo Grubbs normalassimétrico e (b) modelo Grubbs normal.

(a)

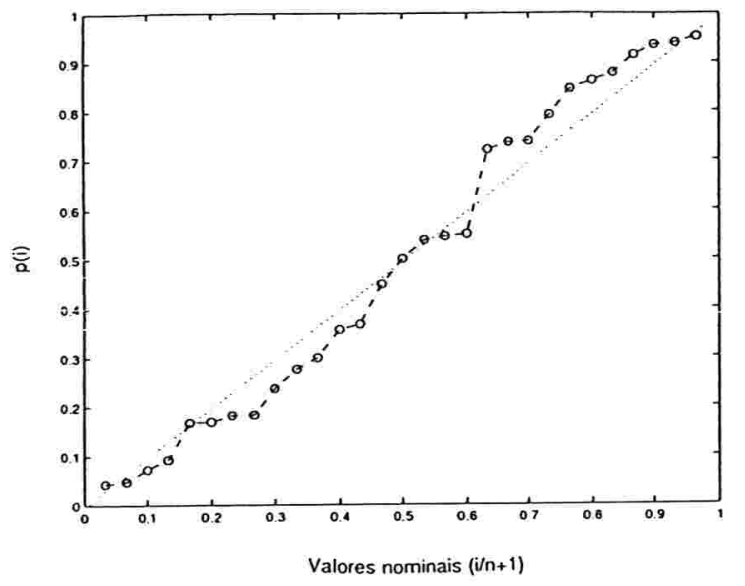

(b)

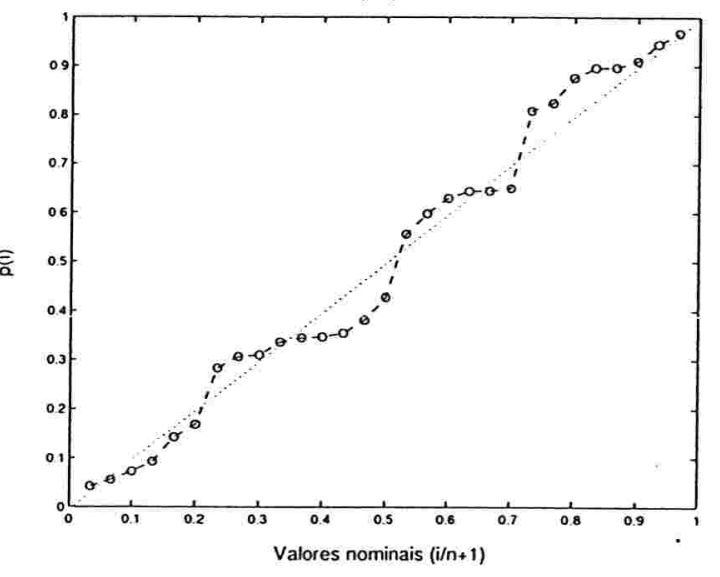


Figura 3.2: Dados de Grubbs, interesse em $\theta$, perturbação de casos. Gráfico de índice de (a) $|d \max |$, (b) $C_{i}$, (c) afastamento da verossimilhança $L D_{i}$ e (d) distância de Cook $D_{i}$.

(a)

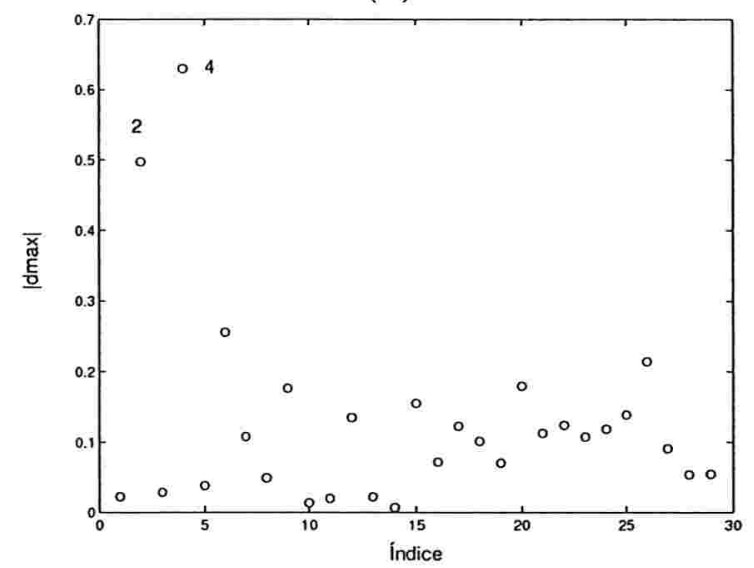

(c)

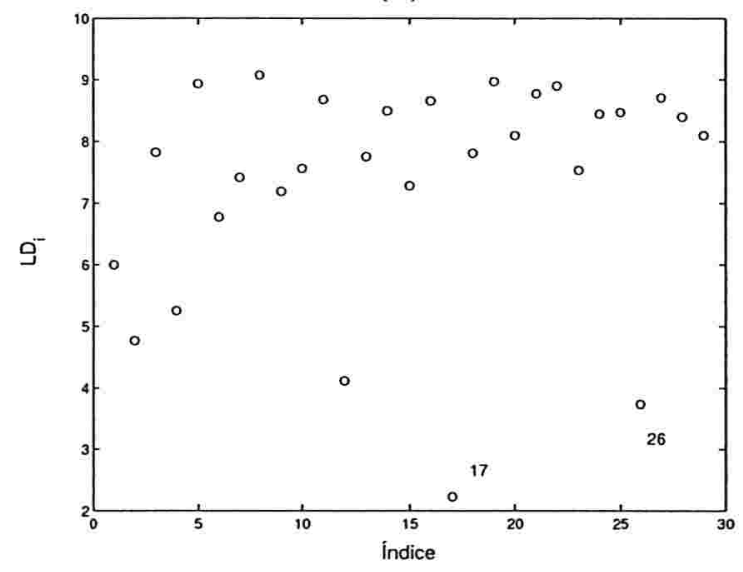

(b)

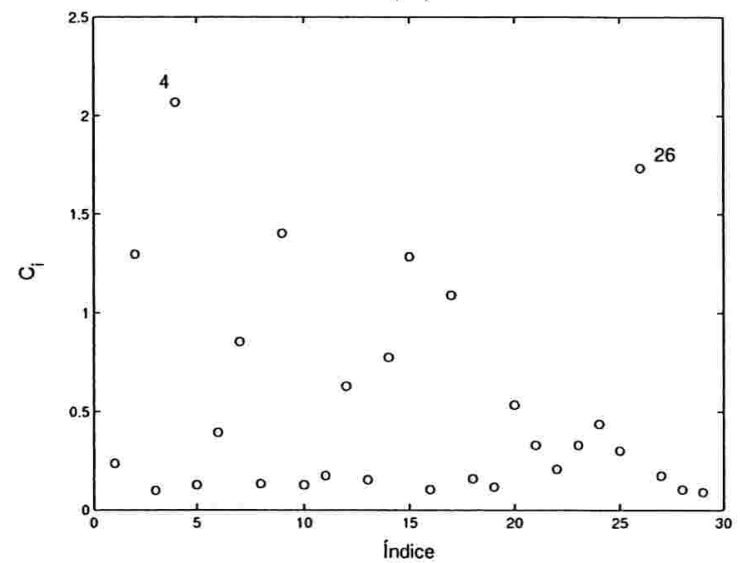

(d)

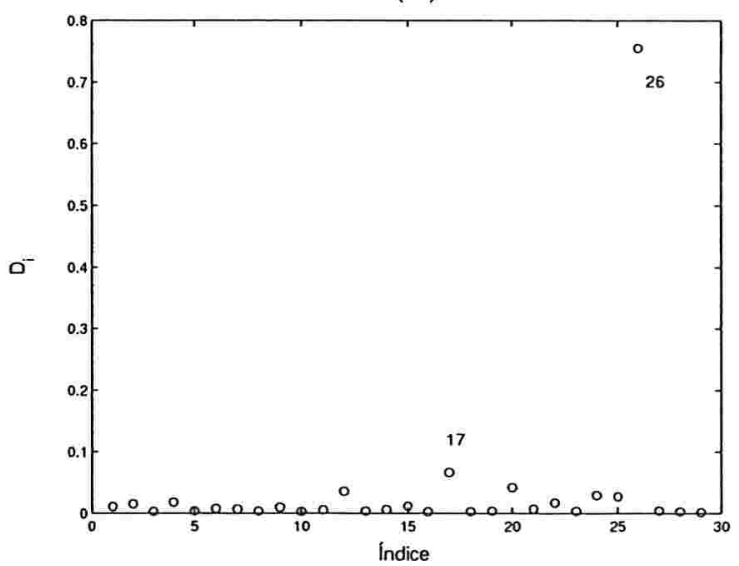


Figura 3.3: Dados de Grubbs, interesse em $\boldsymbol{\theta}$. Gráfico de índice de $|d m a x|$ para (a) Perturbação aditiva e (b) perturbação multiplicativa das medidas obtidas pelo observador A.

(a)

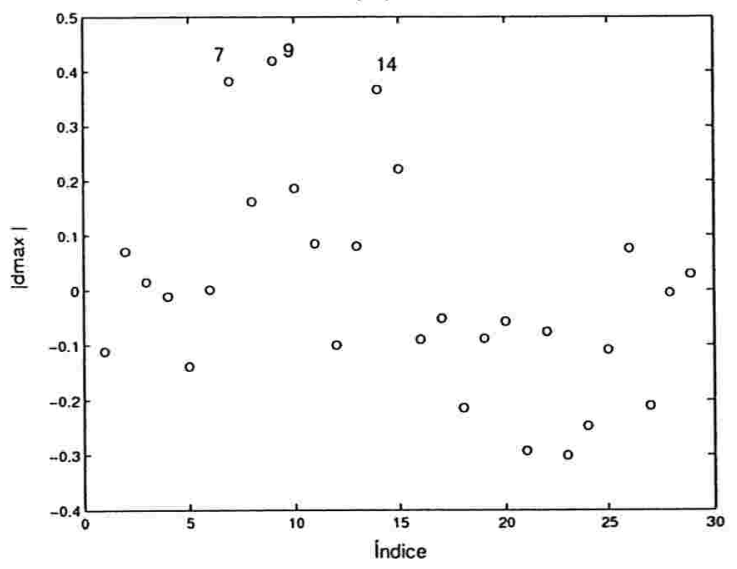

(b)

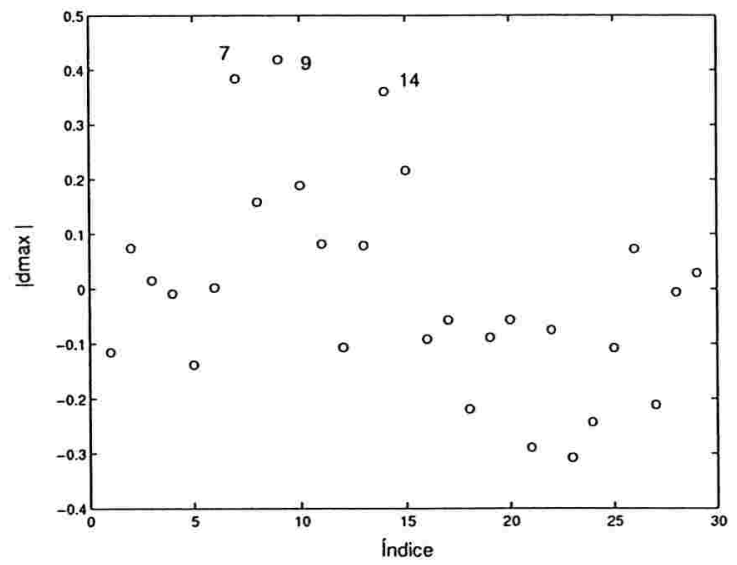

Figura 3.4: Diagnóstico dos dados de Grubbs para MGN, interesse em $\boldsymbol{\theta}$. Gráfico de índice de (a) $C_{i}$ para a perturbação de ponderação de casos e (b) distância de $\operatorname{Cook} D_{i}$.

(a)

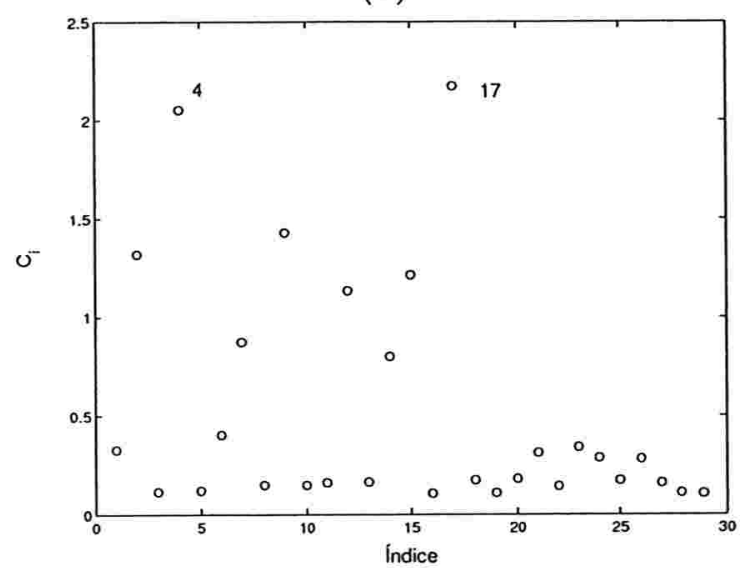

(b)

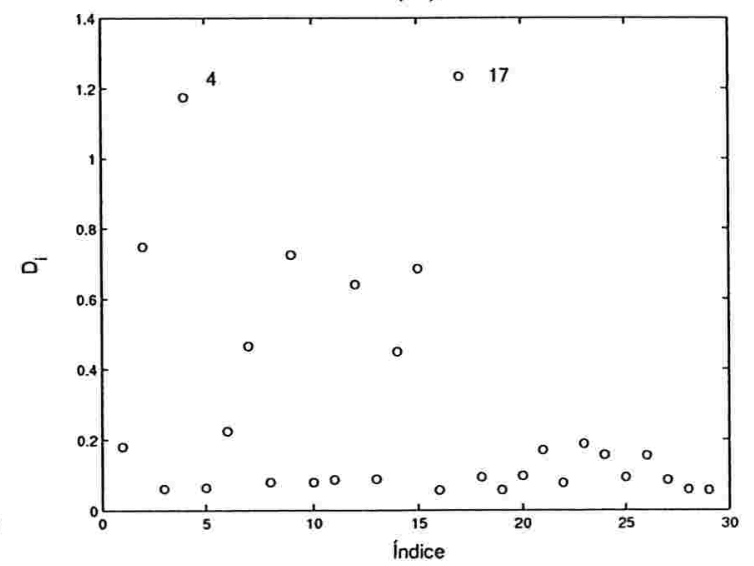


Tabela 3.4: Resultados do ajuste do modelo de Grubbs normal-assimétrico (MGN e MGSN) para os dados de Barnett (1969). EP são estimativas dos erros padrão assintóticos baseados na matriz de informação de Fisher observada dada no Apêndice E.1.

\begin{tabular}{|c|c|c|c|c|}
\hline \multirow[b]{2}{*}{ Parâmetros } & \multicolumn{2}{|c|}{ MGN } & \multicolumn{2}{|c|}{ MGSN } \\
\hline & Estimativas & $\mathrm{EP}$ & Estimativas & $\mathrm{EP}$ \\
\hline$\mu_{x}$ & 22.4611 & 0.9711 & 12.1559 & 1.3918 \\
\hline$\alpha_{2}$ & -0.7042 & 0.2984 & -0.7042 & 0.2961 \\
\hline$\alpha_{3}$ & -0.9750 & 0.3610 & -0.9750 & 0.3649 \\
\hline$\alpha_{4}$ & -1.4389 & 0.3657 & -1.4389 & 0.3693 \\
\hline$\phi_{x}$ & 62.9065 & 10.6442 & 168.9100 & 39.9448 \\
\hline$\phi_{1}$ & 4.9979 & 0.9947 & 5.0611 & 0.9888 \\
\hline$\phi_{2}$ & 1.4129 & 0.5698 & 1.2516 & 0.5640 \\
\hline$\phi_{3}$ & 4.3831 & 0.9742 & 4.5264 & 0.9997 \\
\hline$\phi_{4}$ & 4.6330 & 1.0321 & 4.7577 & 1.0646 \\
\hline$\lambda_{x}$ & - & - & 5.6763 & 3.8284 \\
\hline -log-verossimilhança & \multicolumn{2}{|c|}{-797.6962} & \multicolumn{2}{|c|}{-792.8011} \\
\hline AIC & \multicolumn{2}{|c|}{806.6962} & \multicolumn{2}{|c|}{802.8011} \\
\hline $\mathrm{BIC}$ & \multicolumn{2}{|c|}{823.1795} & \multicolumn{2}{|c|}{821.1159} \\
\hline $\mathrm{HQ}$ & \multicolumn{2}{|c|}{805.4990} & \multicolumn{2}{|c|}{801.4708} \\
\hline
\end{tabular}


Figura 3.5: Dados de Barnett. Gráficos PP tipo Healy para (a) modelo Grubbs normal-assimétrico e (b) modelo Grubbs normal.

(a)

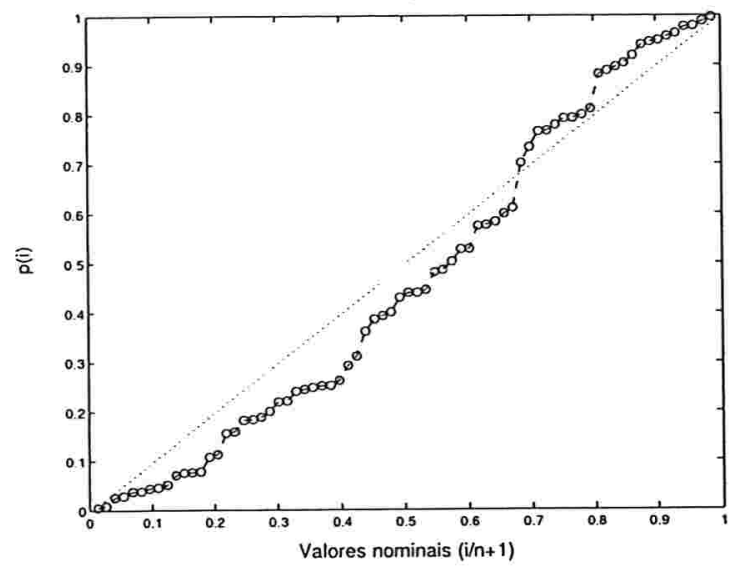

(b)

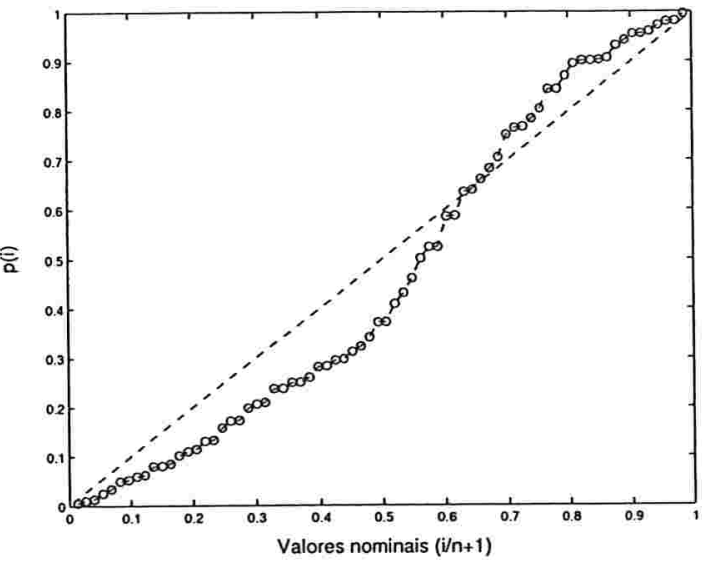

Figura 3.6: Dados de Barnett. Gráfico de índice de (a) $\left|d_{\max }\right|$ e (b) $C_{i}$ para a perturbação de viés multiplicativo.

(a)

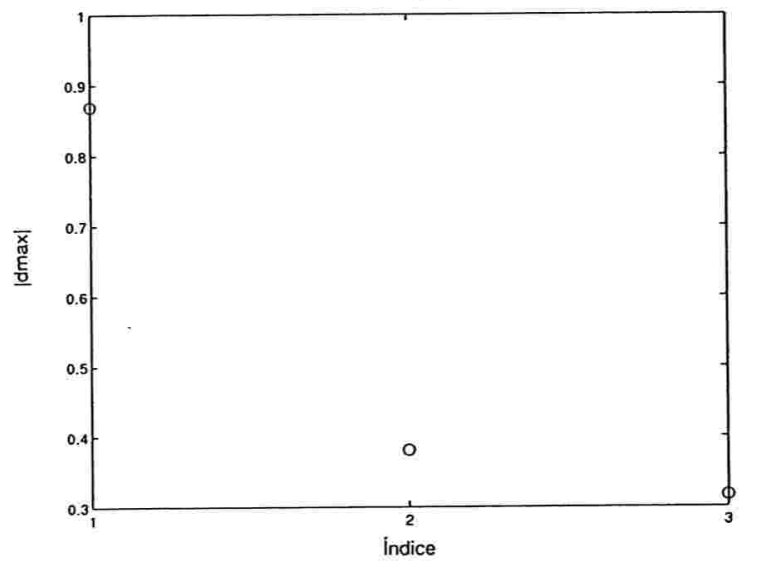

(b)

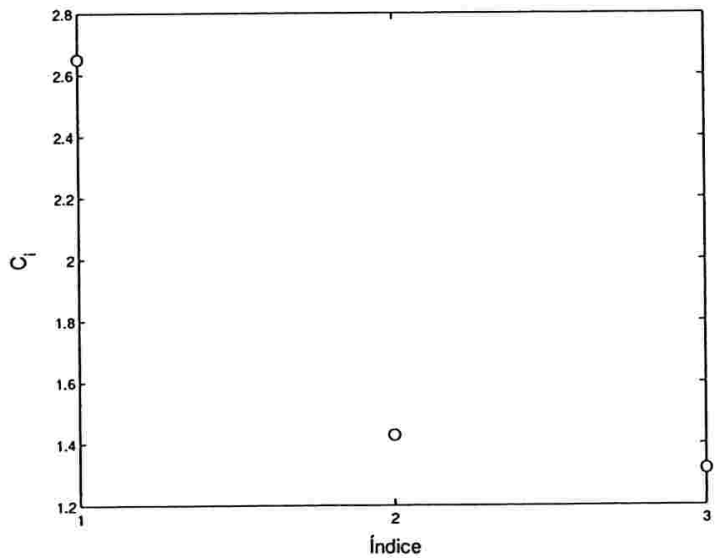




\section{Capítulo 4}

\section{Modelo com intercepto nulo com}

\section{erros de medida normal-assimé-}

\section{trico}

\subsection{Introdução}

No presente capítulo apresentamos o estudo do modelo com intercepto nulo com erro de medida normal-assimétrico. A motivação para considerar tal modelo está ligada ao conjunto de dados da área odontológica encontrado em Hadgu e Koch (1999). Este estudo objetiva comparar dois tipos de enxágüei bucal A, B ou controle avaliando a eficácia na remoção de placa dentária antes (x) e depois (y) do uso do enxágüe bucal. Note-se que, o índice de placa dentária antes (x) do enxágüe bucal segue um comportamento assimétrico. Desta forma, neste capítulo abordamos aspectos inferenciais para modelos com erros de medida com intercepto nulo para uma e duas populações, 
quando a covariável $x$ (variável latente) segue uma distribuição normal-assimétrica (MINSN). Para uma população, a estimação dos parâmetros é calculada pelo método de máxima verossimilhança via algoritmo EM. São apresentadas expressões da função de verossimilhança, da função escore e a matriz de informação de Fisher observada.

Com a finalidade de estudar algumas técnicas de diagnóstico nesta classe de modelos, obtemos matrizes apropriadas para avaliar influência local sobre estimativas dos parâmetros. Uma aplicação é apresentada, utilizando parte do conjunto de dados de Hadgu e Koch (1999). Para o modelo com intercepto nulo com erros de medida normal-assimétrica de duas populações, é apresentada apenas a estimação de máxima verossimilhança via algoritmo EM.

\subsection{Especificação do modelo para uma população}

$\mathrm{Na}$ literatura o modelo de regressão com intercepto nulo com erro de medida é definido por

$$
\begin{aligned}
& X_{i j}=x_{i j}+u_{i j} \\
& y_{i j}=\beta_{i} x_{i j}+\epsilon_{i j}
\end{aligned}
$$

em que $X_{i j}$ e $y_{i j}$, denotam, respectivamente, os valores observados da covariável e da variável resposta para o grupo $i$ e o indivíduo $j,\left(i=1, \ldots, p, j=1, \ldots, n_{i}\right), x_{i j}$, corresponde ao verdadeiro valor da covariável de $X_{i j}, \beta_{i}, i=1, \ldots, p$, parâmetros de inclinação considerados desconhecidos. Usualmente, supõe-se que $x_{i j}, \epsilon_{i j}$ e $u_{i j}$ são mutuamente independentes, com distribuições $N\left(\mu_{x}, \phi_{x}\right), N\left(0, \sigma_{i}^{2}\right), i=1, \ldots, p$, e $N\left(0, \sigma_{u}^{2}\right)$, respectivamente. Embora a suposição de normalidade (ou simetria) seja adequada em muitas situações, não é apropriada quando os dados apresen- 
tem comportamento assimétrico. Neste caso, o conjunto de dados de Hadgu e Koch (1999) pode requerer transformação a fim de melhorar a aproximação pela distribuição normal. Desta forma, o principal objetivo deste capítulo é estudar inferência e diagnóstico no modelo de regressão com intercepto nulo definido por (4.1) e (4.2), com a suposição de que a quantidade desconhecida $x_{i j}$ (variável latente) segue uma distribuição normal-assimétrica univariada. Assim, o vetor de observações, $\mathbf{z}_{i j}=\left(X_{i j}, y_{i j}\right)^{\top}, i=1, \ldots, p, j=1, \ldots, n_{i}$, segue uma distribuição normal-assimétrica bivariada.

Para especificar o modelo de intercepto nulo com erro de medida normal-assimétrico (MINSN), note que podemos escrever o modelo linear (4.1) e (4.2) em forma matricial como

$$
\mathrm{z}_{i j}=\boldsymbol{\beta}_{o i} x_{i j}+\zeta_{i j}
$$

em que $\mathbf{z}_{i j}=\left(X_{i j}, y_{i j}\right)^{\top}$ (vetor de observações) e $\boldsymbol{\beta}_{o i}=\left(1, \beta_{i}\right)^{\top}$ são vetores de dimensão $2 \times 1 ; \zeta_{i j}=\left(u_{i j}, \epsilon_{i j}\right)^{\top}$ é um vetor aleatório de dimensão $2 \times 1$, sendo este chamado MINSN se

$$
\zeta_{i j} \stackrel{i n d}{\sim} N_{2}\left(0, \mathrm{D}^{-1}(\phi)\right) \text { e } x_{i j} \stackrel{i i d}{\sim} S N_{1}\left(\mu_{x}, \phi_{x}, \lambda_{x}\right)
$$

$i=1, \ldots, p, j=1, \ldots, n_{i}, \operatorname{com} \mathrm{D}^{-1}(\phi)=\operatorname{diag}\left(\phi_{i}\right)$ e $\phi_{i}=\left(\sigma_{u}^{2}, \sigma_{i}^{2}\right)^{\top}$. O modelo acima é considerado, para o caso do conjunto de dados de Hadgu e Koch (1999) referente ao índice de placa, o qual não parece ser distribuido simetricamente na população. Por outro lado, os erros $\zeta_{i j}$ são os erros de medida, sendo razoável supor que sejam distribuídos normalmente. O parâmetro de assimetria $\lambda_{x}$ acarreta assimetria na variável latente $x_{i j}$ e, conseqüentemente, nas quantidades observadas $\mathbf{z}_{i j}, i=1, \ldots, p, j=1, \ldots, n_{i}$, podendo ser mostrado que marginalmente segue uma distribuição normal-assimétrica bivariada (Teorema 4.1). Se $\lambda_{x}=0$, então o modelo assimétrico reduz-se ao modelo de intercepto nulo com erro de medida (MINN). 
Note-se que a partir de (2.16) o modelo definido em (4.3) e (4.4), pode ser escrito hierarquicamente, como

$$
\begin{aligned}
\mathbf{z}_{i j} \mid x_{i j} & \stackrel{\text { ind }}{\sim} N_{2}\left(\boldsymbol{\beta}_{o i} x_{i j}, \mathrm{D}^{-1}(\phi)\right), \\
x_{i j} \mid T_{i j}=t_{i j} & \stackrel{i n d}{\sim} N_{1}\left(\mu_{x}+\phi_{x}^{1 / 2} \delta_{x} t_{i j}, \phi_{x}\left(1-\delta_{x}^{2}\right)\right), \\
T_{i j} & \stackrel{i i d}{\sim} H N(0,1),
\end{aligned}
$$

$i=1, \ldots, p, j=1, \ldots, n_{i}$, todos independentes, onde $H N(0,1)$ denota a distribuição half-normal (ou normal truncada a esquerda do zero) univariada padronizada e $\delta_{x}=$ $\lambda_{x} /\left(1+\lambda_{x}^{2}\right)^{1 / 2}$. Inferência clássica sobre o vetor de parâmetros $\boldsymbol{\theta}=\left(\mu_{x}, \boldsymbol{\beta}^{\top}, \phi_{x}, \sigma_{1}^{2}, \ldots\right.$, $\left.\sigma_{p}^{2}, \sigma_{u}^{2}, \lambda_{x}\right)^{\top}$, com $\boldsymbol{\beta}=\left(\beta_{1}, \ldots, \beta_{p}\right)^{\top}$, é baseado na distribuição marginal para a resposta $\mathbf{z}_{i j}$, dada no seguinte teorema:

Teorema 4.1. Sob o modelo definido em (4.5)-(4.7), a distribuição marginal de $\mathbf{z}_{i j}$ é dada por

$$
f_{\mathbf{z}_{i j}}\left(\mathbf{z}_{i j} \mid \boldsymbol{\theta}\right)=2 \phi_{2}\left(\mathbf{z}_{i j} \mid \mu_{i}, \Sigma_{i}\right) \Phi_{1}\left(\bar{\lambda}_{i x}^{\top} \Sigma_{i}^{-1 / 2}\left(\mathbf{z}_{i j}-\mu_{i}\right)\right)
$$

$i=1, \ldots, p, j=1, \ldots, n_{i}$, isto é, $\mathbf{z}_{i j} \stackrel{\text { ind }}{\sim} S N_{2}\left(\boldsymbol{\mu}_{i}, \boldsymbol{\Sigma}_{i}, \bar{\lambda}_{i}\right), \operatorname{com} \boldsymbol{\mu}_{i}=\boldsymbol{\beta}_{o i} \mu_{x}, \boldsymbol{\Sigma}_{i}=$ $D\left(\phi_{i}\right)+\phi_{x} \boldsymbol{\beta}_{o i} \boldsymbol{\beta}_{o i}^{\top}, \quad \overline{\boldsymbol{\lambda}}_{i}=\frac{\lambda_{x} \phi_{x} \Sigma_{i}^{-1 / 2} \boldsymbol{\beta}_{o i}}{\sqrt{\phi_{x}+\lambda_{x}^{2} \Lambda_{i}}}$, em que $\Lambda_{i}=\left(\phi_{x}^{-1}+\boldsymbol{\beta}_{o i}^{\top} D\left(\phi_{i}\right)^{-1} \boldsymbol{\beta}_{o i}\right)^{-1}$.

Demonstração. A prova deste resultado é similar à prova do Teorema 3.1 (ver também Lachos, 2004).

\subsubsection{Função Escore}

De (4.8), e depois de algumas manipulações algebricas a função de log-verossimilhança pode ser alternativamente escrita como:

$$
\ell(\boldsymbol{\theta})=\sum_{i=1}^{p} \sum_{j=1}^{n_{i}} \ell_{i j}(\boldsymbol{\theta})
$$


em que $\ell_{i j}(\boldsymbol{\theta})=-\frac{1}{2} \log \left|\Sigma_{i}\right|-\frac{1}{2} g_{i j}+\log \left(K_{i j}\right)+C$, onde C denota uma constante que não depende do vetor de parâmetro $\theta, g_{i j}=\left(\mathbf{z}_{i j}-\boldsymbol{\mu}_{i}\right)^{\top} \boldsymbol{\Sigma}_{i}^{-1}\left(\mathbf{z}_{i j}-\boldsymbol{\mu}_{i}\right)$ e $K_{i j}=$ $\Phi_{1}\left(A_{i} a_{i j}\right), \operatorname{com} A_{i}=\frac{\lambda_{x} \Lambda_{i}}{\sqrt{\phi_{x}+\lambda_{x}^{2} \Lambda_{i}}}, a_{i j}=\left(\mathbf{z}_{i j}-\mu_{i}\right)^{\top} D^{-1}\left(\phi_{i}\right) \boldsymbol{\beta}_{o i}, \Lambda_{i}=\frac{\phi_{x}}{c_{i}}$ e $c_{i}=$ $1+\phi_{x} \boldsymbol{\beta}_{o i}^{\top} D^{-1}\left(\phi_{i}\right) \boldsymbol{\beta}_{o i}, i=1, \ldots, p, j=1, \ldots, n_{i}$. A função escore é dada por

$$
U(\boldsymbol{\theta})=\frac{\partial \ell(\boldsymbol{\theta})}{\partial \boldsymbol{\theta}}=\sum_{i=1}^{p} \sum_{j=1}^{n_{i}} U_{i j}(\boldsymbol{\theta}),
$$

em que

$$
\begin{aligned}
& U_{i j}(\boldsymbol{\theta})=\frac{\partial \ell_{i j}(\boldsymbol{\theta})}{\partial \boldsymbol{\theta}}=\left(U_{i j}\left(\mu_{x}\right), U_{i j}\left(\beta_{1}\right), \ldots, U_{i j}\left(\beta_{p}\right), U_{i j}\left(\phi_{x}\right), U_{i j}\left(\sigma_{1}^{2}\right), \ldots, U_{i j}\left(\sigma_{p}^{2}\right), U_{i j}\left(\sigma_{u}^{2}\right),\right. \\
& \left.U_{i j}\left(\lambda_{x}\right)\right)^{\top}
\end{aligned}
$$

$\mathrm{e}$

$$
U_{i j}(\gamma)=\frac{\partial \ell_{i j}(\theta)}{\partial \gamma}=-\frac{1}{2} \frac{\partial \log \left|\Sigma_{i}\right|}{\partial \gamma}-\frac{1}{2} \frac{\partial g_{i j}}{\partial \gamma}+\frac{\partial \log K_{i j}}{\partial \gamma}
$$

em que $\frac{\partial \log K_{i j}}{\partial \gamma}=W_{\Phi_{1}}\left(A_{i} a_{i j}\right)\left\{A_{i} \frac{\partial a_{i j}}{\partial \gamma}+a_{i j} \frac{\partial A_{i}}{\partial \gamma}\right\}$, com $W_{\Phi_{1}}(u)=\phi_{1}(u) / \Phi_{1}(u)$, $u \in \mathbb{R}, \gamma=\mu_{x}, \beta_{1}, \ldots, \beta_{p}, \phi_{x}, \sigma_{1}^{2}, \ldots, \sigma_{p}^{2}, \sigma_{u}^{2}$ e $\lambda_{x}, i=1, \ldots, p, j=1, \ldots, n_{i}$. As expressões destas derivadas encontram-se no Apêndice D.2.

\subsubsection{Matriz de informação de Fisher observada}

A matriz de segundas derivadas em relação a $\theta$ é dada por

$$
\mathbf{L}=\frac{\partial^{2} \ell(\boldsymbol{\theta})}{\partial \boldsymbol{\theta} \partial \boldsymbol{\theta}^{\top}}=\sum_{i=1}^{p} \sum_{j=1}^{n_{i}} \frac{\partial^{2} \ell_{i j}(\boldsymbol{\theta})}{\partial \boldsymbol{\theta} \partial \boldsymbol{\theta}^{\top}} .
$$

De (4.10) segue que o elemento geral da matriz de informação de Fisher observada é dado por

$$
\mathbf{J}_{i j}=-\mathbf{L}_{i j}(\boldsymbol{\theta})=-\frac{\partial^{2} \ell_{i j}(\boldsymbol{\theta})}{\partial \boldsymbol{\gamma} \partial \boldsymbol{\tau}^{\top}}
$$


em que $\frac{\partial^{2} \ell_{i j}}{\partial \gamma \partial \tau^{\top}}=-\frac{1}{2} \frac{\partial^{2} \log \left|\Sigma_{i}\right|}{\partial \gamma \partial \tau^{\top}}-\frac{1}{2} \frac{\partial^{2} g_{i j}}{\partial \gamma \partial \tau^{\top}}+\frac{\partial^{2} \log K_{i j}}{\partial \gamma \partial \tau^{\top}}$, com

$$
\begin{aligned}
\frac{\partial^{2} \log K_{i j}}{\partial \gamma \partial \tau^{\top}}= & W_{\Phi_{1}}\left(A_{i} a_{i j}\right)\left\{\frac{\partial A_{i}}{\partial \gamma} \frac{\partial a_{i j}}{\partial \tau^{\top}}+A_{i} \frac{\partial^{2} a_{i j}}{\partial \gamma \partial \tau^{\top}}+\frac{\partial a_{i j}}{\partial \gamma} \frac{\partial A_{i}}{\partial \tau^{\top}}+a_{i j} \frac{\partial^{2} A_{i}}{\partial \gamma \partial \tau^{\top}}\right\} \\
& +\Delta_{\Phi_{1}}\left(A_{i} a_{i j}\right)\left\{A_{i} \frac{\partial a_{i j}}{\partial \gamma}+a_{i j} \frac{\partial A_{i}}{\partial \gamma}\right\}\left\{A_{i} \frac{\partial a_{i j}}{\partial \tau^{\top}}+a_{i j} \frac{\partial A_{i}}{\partial \tau^{\top}}\right\}
\end{aligned}
$$

$\Delta_{\Phi_{1}}(u)=W_{\Phi_{1}}^{\prime}(u)=-W_{\Phi_{1}}(u) u+W_{\Phi_{1}}(u), u \in \mathbb{R}, \gamma, \tau=\mu_{x}, \beta_{1}, \ldots, \beta_{p}, \phi_{x}, \sigma_{1}^{2}, \ldots$, $\sigma_{p}^{2}, \sigma_{u}^{2}$ e $\lambda_{x}$. Estas derivadas são apresentadas no Apêndice E.2.

Intervalos de confiança assintóticos e testes baseados nos EMV's podem ser obtidos usando esta matriz. Na seguinte seção discutimos o EMV do vetor de parâmetros $\boldsymbol{\theta}$ usando o algoritmo EM. Note que métodos como o de Newton-Raphson e quaseNewton, podem ser implementados usando os resultados acima.

\subsubsection{Estimação de máxima verossimilhança via algoritmo EM}

Utilizando a definição do algoritmo EM visto na Seção 2.3 para o modelo MINSN consideramos que $\mathbf{z}=\left(\mathbf{z}_{11}^{\top}, \ldots, \mathbf{z}_{1 n_{1}}^{\top}, \ldots, \mathbf{z}_{p 1}^{\top}, \ldots, \mathbf{z}_{p n_{p}}^{\top}\right)^{\top}$, com $\mathbf{z}_{i j}=\left(y_{i j}, X_{i j}\right), i=1, \ldots, p$, $j=1, \ldots, n_{i}, \mathbf{x}=\left(x_{11}, \ldots, x_{1 n_{1}}, \ldots, x_{p 1}, \ldots, x_{p n_{p}}\right)^{\top}$ e $\mathbf{t}=\left(t_{11}, \ldots, t_{1 n_{1}}, \ldots, t_{p 1}, \ldots\right.$, $\left.t_{p n_{p}}\right)^{\top}$. A seguir implementamos o algoritmo EM para o MINSN usando dados duplamente aumentados, ou seja, consideramos que (x,t) em (4.5)-(4.7) são dados latentes. Sob a representação hierárquica (4.5)-(4.7), com $v^{2}=\phi_{x}\left(1-\delta_{x}^{2}\right)$ e $\tau=\phi_{x}^{1 / 2} \delta_{x}$, a função 
de log-verossimilhança completa associada com $(\mathbf{z}, \mathbf{x}, \mathbf{t})$ é

$$
\begin{aligned}
\ell_{c}(\boldsymbol{\theta} \mid \mathbf{z}, \mathbf{x}, \mathbf{t})= & -\sum_{i=1}^{p} \frac{n_{i}}{2} \log \left(\left|D\left(\phi_{i}\right)\right|\right)-\frac{1}{2} \sum_{i=1}^{p} \sum_{j=1}^{n_{i}}\left(\mathbf{z}_{i j}-\boldsymbol{\beta}_{o i} x_{i j}\right)^{\top} D^{-1}\left(\phi_{i}\right) \\
& \left(\mathbf{z}_{i j}-\boldsymbol{\beta}_{o i} x_{i j}\right)-\frac{N}{2} \log \left(v^{2}\right)-\frac{1}{2 v^{2}} \sum_{i=1}^{p} \sum_{j=1}^{n_{i}}\left(x_{i j}-\mu_{x}-\tau t_{i j}\right)^{2} \\
& -\frac{1}{2} \sum_{i=1}^{p} \sum_{j=1}^{n_{i}} t_{i j}^{2}+C
\end{aligned}
$$

em que $N=\sum_{i=1}^{p} n_{i}$ e $C$ é uma constante que independe do vetor de parâmetros $\theta$. Dessa forma, o algoritmo EM compreende os seguintes passos:

Passo-E: Dado $\boldsymbol{\theta}=\widehat{\boldsymbol{\theta}}$. Sejam $\widehat{x}_{i j}=E\left[x_{i j} \mid \boldsymbol{\theta}=\widehat{\boldsymbol{\theta}}, \mathrm{z}_{i j}\right], \widehat{x_{i j}^{2}}=E\left[x_{i j}^{2} \mid \boldsymbol{\theta}=\widehat{\boldsymbol{\theta}}, \mathrm{z}_{i j}\right]$, $\widehat{t}_{i j}=E\left[T_{i j} \mid \boldsymbol{\theta}=\widehat{\boldsymbol{\theta}}, \mathbf{z}_{i j}\right], \widehat{t}_{i j}^{2}=E\left[T_{i j}^{2} \mid \boldsymbol{\theta}=\widehat{\boldsymbol{\theta}}, \mathbf{z}_{i j}\right]$ e $\widehat{t x}_{i j}=E\left[T_{i j} x_{i j} \mid \boldsymbol{\theta}=\widehat{\boldsymbol{\theta}}, \mathbf{z}_{i j}\right]$, calculamos $\widehat{t}_{i j}, \widehat{t}^{2}{ }_{i j}, \widehat{x}_{i j}, \widehat{x}_{i j}^{2}$ e $\widehat{t x}_{i j}$ para $i=1, \ldots, p$ e $j=1, \ldots, n_{i}$, que são calculadas de forma similar ao MGSN (ver Apêndice C), obtendo-se

$$
\begin{aligned}
& \widehat{t}_{i j}=\widehat{\mu}_{T_{i j}}+W_{\Phi_{1}}\left(\frac{\widehat{\mu}_{T_{i j}}}{\widehat{N}_{T i}}\right) \widehat{N}_{T i}, \\
& \widehat{t}_{i j}^{2}=\widehat{\mu}_{T_{i j}}^{2}+\widehat{N}_{T i}^{2}+W_{\Phi_{1}}\left(\frac{\widehat{\mu}_{T_{i j}}}{\widehat{N}_{T i}}\right) \widehat{N}_{T i} \widehat{\mu}_{T_{i j}}, \\
& \widehat{x}_{i j}=\widehat{c}_{i j}+\widehat{d}_{i} \widehat{t}_{i j}, \\
& \widehat{x_{i j}^{2}}=\widehat{M^{2}}{ }_{i}+\widehat{c}_{i j}^{2}+2 \widehat{c}_{i j} \widehat{d}_{i} \widehat{t}_{i j}+\widehat{d}_{i}^{2} \widehat{t}_{i j}^{2}
\end{aligned}
$$

e

$$
\widehat{t x}_{i j}=\widehat{c}_{i j} \widehat{t}_{i j}+\widehat{d}_{i} \widehat{t}_{i j}^{2}
$$

em que $\widehat{\mu}_{T_{i j}}=\widehat{\tau} \widehat{N}_{T i}^{2} \widehat{\boldsymbol{\beta}}_{o i}^{\top}\left(D\left(\widehat{\boldsymbol{\phi}}_{i}\right)+\widehat{v}^{2} \widehat{\boldsymbol{\beta}}_{o i} \widehat{\boldsymbol{\beta}}_{o i}^{\top}\right)^{-1}\left(\mathbf{z}_{i j}-\widehat{\boldsymbol{\beta}}_{o i} \widehat{\mu}_{x}\right)$, $\widehat{N}_{T i}^{2}=\left[1+\widehat{\tau}^{2} \widehat{\boldsymbol{\beta}}_{o i}^{\top}\left(D\left(\widehat{\boldsymbol{\phi}}_{i}\right)+\widehat{v}^{2} \widehat{\boldsymbol{\beta}}_{o i} \widehat{\boldsymbol{\beta}}_{o i}^{\top}\right)^{-1} \widehat{\boldsymbol{\beta}}_{o i}\right]^{-1}, \widehat{M}_{i}^{2}=\widehat{v}^{2}\left[1+\widehat{v}^{2} \widehat{\boldsymbol{\beta}}_{o i}^{\top} D^{-1}\left(\widehat{\boldsymbol{\phi}}_{i}\right) \widehat{\boldsymbol{\beta}}_{o i}\right]^{-1}$, $\widehat{c}_{i j}=\widehat{\mu}_{x}+\widehat{M}_{i}^{2} \widehat{\boldsymbol{\beta}}_{o i}^{\top} D^{-1}\left(\widehat{\phi}_{i}\right)\left(\mathbf{z}_{i j}-\widehat{\boldsymbol{\beta}}_{o i} \widehat{\mu}_{x}\right)$ e $\widehat{d}_{i}=\widehat{\tau}\left(1-\widehat{M}_{i}^{2} \widehat{\boldsymbol{\beta}}_{o i}^{\top} D^{-1}\left(\widehat{\phi}_{i}\right) \widehat{\boldsymbol{\beta}}_{o i}\right)$.

Conseqüentemente, a esperança condicional correspondente a função $Q(. \mid \widehat{\boldsymbol{\theta}})$ tem a 
seguinte forma:

$$
\begin{aligned}
Q(. \mid \widehat{\boldsymbol{\theta}})= & E\left[\ell_{c}(\boldsymbol{\theta} \mid \mathbf{z}, \mathbf{x}, \mathbf{t}) \mid \mathbf{z}, \widehat{\theta}\right]=-\sum_{i=1}^{p} \frac{n_{i}}{2} \log \left(\left|D\left(\phi_{i}\right)\right|\right) \\
& -\frac{1}{2} \sum_{i=1}^{p} \sum_{j=1}^{n_{i}}\left(\mathbf{z}_{i j}-\boldsymbol{\beta}_{0 i} \widehat{x}_{i j}\right)^{\top} D^{-1}\left(\phi_{i}\right)\left(\mathbf{z}_{i j}-\boldsymbol{\beta}_{o i} \widehat{x}_{i j}\right) \\
& -\frac{1}{2} \sum_{i=1}^{p} \sum_{j=1}^{n_{i}}\left(\widehat{x_{i j}^{2}}-\left(\widehat{x}_{i j}\right)^{2}\right) \boldsymbol{\beta}_{o i}^{\top} \mathrm{D}^{-1}(\phi) \boldsymbol{\beta}_{o i} \\
& -\frac{N}{2} \log \left(v^{2}\right)-\frac{1}{2 v^{2}} \sum_{i=1}^{p} \sum_{j=1}^{n_{i}} \widehat{\left(x_{i j}^{2}\right.}+\mu_{x}^{2}+\tau^{2} \widehat{t}_{i j}^{2} \\
& \left.-2 \widehat{x}_{i j} \mu_{x}-2 \tau \widehat{t x_{i j}}+2 \tau \mu_{x} \widehat{t}_{i j}\right) .
\end{aligned}
$$

Passo-M: Atualizar $\widehat{\boldsymbol{\theta}}$ maximizando $E\left[\ell_{c}(\boldsymbol{\theta} \mid \mathbf{z}, \mathbf{x}, \mathbf{t}) \mid \mathbf{z}, \widehat{\boldsymbol{\theta}}\right]$ sob $\boldsymbol{\theta}$. Obtemos os seguintes resultados:

$$
\begin{aligned}
& \widehat{\mu}_{x}=\frac{1}{N} \sum_{i=1}^{p} \sum_{j=1}^{n_{i}}\left(\widehat{x}_{i j}-\tau \widehat{t}_{i j}\right), \\
& \widehat{\beta}_{i}=\frac{\sum_{j=1}^{n_{i}} y_{i j} \widehat{x}_{i j}}{\sum_{j=1}^{n_{i}} \widehat{x_{i j}^{2}}}, i=1, \ldots, p \\
& \widehat{\sigma}_{i}^{2}=\frac{1}{n_{i}}\left[\sum_{j=1}^{n_{i}} y_{i j}^{2}-\frac{\left(\sum_{j=1}^{n_{i}} y_{i j} \widehat{x}_{i j}\right)^{2}}{\sum_{j=1}^{n_{i}} \widehat{x_{i j}^{2}}}\right], i=1, \ldots, p \\
& \widehat{\sigma}_{u}^{2}=\frac{1}{N} \sum_{i=1}^{p} \sum_{j=1}^{n_{i}}\left(X_{i j}^{2}-2 X_{i j} \widehat{x}_{i j}+{\widehat{x^{2}}}_{i j}\right) \text {, } \\
& \widehat{v}^{2}=\frac{1}{N} \sum_{i=1}^{p} \sum_{j=1}^{n_{i}}\left(\widehat{x_{i j}^{2}}+\mu_{x}^{2}+\tau^{2} \widehat{t}_{i j}^{2}-2 \mu_{x} \widehat{x}_{i j}-2 \tau \widehat{t x}_{i j}+2 \tau \mu_{x} \widehat{t}_{i j}\right), \\
& \text { e } \\
& \widehat{\tau}=\frac{\sum_{i=1}^{p} \sum_{j=1}^{n_{i}}\left(\widehat{t x}_{i j}-\mu_{x} \widehat{t}_{i j}\right)}{\sum_{i=1}^{p} \sum_{j=1}^{n_{i}} \widehat{t}^{2}} .
\end{aligned}
$$

Note que $\widehat{\beta}_{i}$ pode ser escrita, alternativamente, como $\widehat{\boldsymbol{\beta}}=\Delta_{x}^{-1} \delta_{x y}$, em que $\Delta_{x}=$ $\operatorname{diag}\left(\sum_{j=1}^{n_{1}} \widehat{x_{1 j}^{2}}, \ldots, \sum_{j=1}^{n_{p}}{\widehat{x^{2}}}_{p j}\right)$ e $\delta_{x y}=\left(\sum_{j=1}^{n_{1}} y_{1 j} \widehat{x}_{1 j}, \ldots, \sum_{j=1}^{n_{p}} y_{p j} \widehat{x}_{p j}\right)^{\top}$. Os parâmetros de forma e escala da variável latente $x$ podem ser estimados levando em conta que 
$\tau / v=\lambda_{x}$ e $\phi_{x}=\tau^{2}+v^{2}$. Os valores iniciais podem geralmente ser escolhidos como sendo as correspondentes estimativas sob a suposição de normalidade, em que o parâmetro de assimetria é igual a 0 e, como recomendado na literatura, é prudente executar o algoritmo EM várias vezes com diferentes valores iniciais. Para a seleção dos modelos propomos avaliar a série de ajustes inspecionando critérios de informação definido na Seção 2.4. Estes critérios podem ser usados na prática para selecionar entre os modelos MINN e MINSN ajustados.

\subsection{Especificação do modelo para duas populações}

Recentemente, Aoki et al. (2003), do ponto de vista bayesiano estudaram o modelo de intercepto nulo com erro de medida normal multivariado (MINMN) com uma estrutura de dependência na variável resposta em unidades do mesmo grupo. Nesta seção propomos estender para duas populações o modelo normal multivariado considerando que a variável latente segue uma distribuição normal-assimétrica (MINMSN), ou seja, assumimos que

$$
\begin{aligned}
X_{i j} & =x_{i j}+u_{i j}, \\
y_{k i j} & =\beta_{k i} x_{i j}+\epsilon_{k i j},
\end{aligned}
$$

$\operatorname{com} u_{i j} \stackrel{i i d}{\sim} N\left(0, \sigma_{u}^{2}\right), \epsilon_{k i j} \stackrel{i n d}{\sim} N\left(0, \sigma_{\epsilon i}^{2}\right), u_{i j}$ e $\epsilon_{k i j}$ são independentes de $x_{i j} \stackrel{i i d}{\sim} S N\left(\mu_{x}, \phi_{x}\right.$, $\left.\lambda_{x}\right), i=1, \ldots, p, j=1, \ldots, n_{i}, k=1,2$. Definimos $\mathbf{Z}_{i j}=\left(X_{i j}, y_{1 i j}, y_{2 i j}\right)^{\top}$ como o vetor observado. Assim, usando a partir de (2.16) e o modelo definido em (4.11), 
podemos expressar como

$$
\begin{aligned}
\mathbf{Z}_{i j} \mid x_{i j} & \stackrel{i n d}{\sim} N_{3}\left(x_{i j} \boldsymbol{\beta}_{o i}, D\left(\phi_{i}\right)\right), \\
x_{i j} \mid T_{i j}=t_{i j} & \stackrel{i n d}{\sim} N\left(\mu_{x}+\phi_{x}^{1 / 2} \delta_{x} t_{i j}, \phi_{x}\left(1-\delta_{x}^{2}\right)\right), \\
T_{i j} & \stackrel{i i d}{\sim} H N(0,1),
\end{aligned}
$$

$i=1, \ldots, p$ e $j=1, \ldots, n_{i}$, todos independentes, onde $\boldsymbol{\beta}_{o i}=\left(1, \boldsymbol{\beta}_{i}^{\top}\right)^{\top}=\left(1, \beta_{1 i}, \beta_{2 i}\right)^{\top}$, $D\left(\phi_{i}\right)=\operatorname{diagonal}\left(\phi_{i}\right), \phi_{i}=\left(\sigma^{2}, \sigma_{\epsilon i}^{2}, \sigma_{\epsilon i}^{2}\right)^{\top}$ e $\delta_{x}=\lambda_{x} /\left(1+\lambda_{x}^{2}\right)^{1 / 2}$. Inferência clássica sobre o vetor de parâmetro $\boldsymbol{\theta}=\left(\mu_{x}, \phi_{x}, \lambda_{x}, \boldsymbol{\beta}^{\top}, \sigma^{2}, \sigma_{\epsilon}^{2}\right)^{\top}$, com $\boldsymbol{\beta}=\left(\beta_{11}, \ldots, \beta_{1 p}, \beta_{21}\right.$, $\left.\ldots, \beta_{2 p}\right)^{\top}$ e $\sigma_{\epsilon}^{2}=\left(\sigma_{\epsilon 1}^{2}, \ldots, \sigma_{\epsilon p}^{2}\right)^{\top}$, é baseada na distribuição marginal para a resposta $\mathbf{Z}_{i j}$, sendo apresentada no seguinte teorema.

Teorema 4.2. Sob o modelo definido em (4.12)-(4.14), segue que a distribuição marginal de $\boldsymbol{Z}_{i j}$ é dada por

$$
f_{Z_{i j}}\left(z_{i j} \mid \boldsymbol{\theta}\right)=2 \phi_{3}\left(z_{i j} \mid \mu_{i}, \Sigma_{i}\right) \Phi_{1}\left(\bar{\lambda}_{x i}^{\top} \Sigma_{i}^{-1 / 2}\left(z_{i j}-\mu_{i}\right)\right)
$$

$i=1, \ldots, p$ e $j=1, \ldots, n_{i}$, i.e., $\mathbf{Z}_{i j} \stackrel{\text { ind }}{\sim} S N_{3}\left(\mu_{i}, \Sigma_{i}, \bar{\lambda}_{x i}\right)$, com $\boldsymbol{\mu}_{i}=\boldsymbol{\beta}_{\text {oi }} \mu_{x}, \boldsymbol{\Sigma}_{i}=$ $D\left(\phi_{i}\right)+\phi_{x} \boldsymbol{\beta}_{o i} \boldsymbol{\beta}_{o i}^{\top}, \bar{\lambda}_{x i}=\frac{\lambda_{x} \phi_{x} \Sigma_{i}^{-1 / 2} \boldsymbol{\beta}_{o i}}{\sqrt{\phi_{x}+\lambda_{x}^{2} \Lambda_{i}}}$, onde $\Lambda_{i}=\left(\phi_{x}^{-1}+\boldsymbol{\beta}_{o i}^{\top} D^{-1}\left(\phi_{i}\right) \boldsymbol{\beta}_{o i}\right)^{-1} . A$ função de log-verossimilhança para $\boldsymbol{\theta}$, dada uma amostra observada $\mathbf{z}=\left(\mathbf{z}_{1}^{\top}, \ldots, \mathbf{z}_{p}^{\top}\right)^{\top}$, $\operatorname{com} \mathbf{z}_{i}=\left(\mathbf{z}_{i 1}^{\top}, \ldots, \mathbf{z}_{i n_{i}}^{\top}\right)^{\top}$ pode ser escrita como $\ell(\boldsymbol{\theta})=\sum_{i=1}^{p} \sum_{j=1}^{n_{i}} \ell_{i j}(\boldsymbol{\theta})$, onde

$$
\ell_{i j}(\boldsymbol{\theta})=-\frac{1}{2} \log \left|\boldsymbol{\Sigma}_{i}\right|-\frac{1}{2}\left(\mathbf{z}_{i j}-\boldsymbol{\mu}_{i}\right)^{\top} \boldsymbol{\Sigma}_{i}^{-1}\left(\mathbf{z}_{i j}-\boldsymbol{\mu}_{i}\right)+\log \left(K_{i j}\right)+C,
$$

onde $C$ é uma constante que independe do vetor de parâmetro $\boldsymbol{\theta}$ e $K_{i j}=\Phi_{1}\left(\bar{\lambda}_{x i}^{\top} \Sigma_{i}^{-1 / 2}\right.$ $\left.\left(z_{i j}-\mu_{i}\right)\right)$

Demonstração. A prova deste resultado é similar à prova do Teorema 3.1 (ver também, Lachos, 2004). 


\subsubsection{Estimação de máxima verossimilhança via algoritmo EM}

Utilizando a definição do algoritmo EM descrito na Seção 2.3, para o modelo MINMSN, consideramos $\mathbf{z}_{c}=\left(\mathbf{z}^{\top}, \mathbf{x}^{\top}, \mathbf{t}^{\top}\right)^{\top}, \operatorname{com} \mathbf{z}=\left(\mathbf{z}_{1}^{\top}, \ldots, \mathbf{z}_{p}^{\top}\right)^{\top}, \mathbf{x}=\left(\mathbf{x}_{1}^{\top}, \ldots, \mathbf{x}_{p}^{\top}\right)^{\top} \mathrm{e}$ $\mathbf{t}=\left(\mathbf{t}_{1}^{\top}, \ldots, \mathbf{t}_{p}^{\top}\right)^{\top}$, onde $\mathbf{z}_{i}=\left(\mathbf{z}_{i 1}^{\top}, \ldots, \mathbf{z}_{i n_{i}}^{\top}\right)^{\top}, \mathbf{x}_{i}=\left(x_{i 1}, \ldots, x_{i n_{i}}\right)^{\top}$ e $\mathbf{t}_{i}=\left(t_{i 1}, \ldots, t_{i n_{i}}\right)^{\top}$, $i=1, \ldots, p$. A seguir implementamos o algoritmo EM para o MINMSN utilizando dados suplamente aumentados, ou seja, consideremos que $(\mathrm{x}, \mathrm{t})$ são conhecidos como dados perdidos ou latentes. Sob a representação hierárquica (4.12)-(4.14), com $\nu^{2}=\sigma_{x}^{2}\left(1-\delta_{x}^{2}\right)$ e $\varsigma=\sigma_{x} \delta_{x}$, segue que a função de verossimilhança completa associada com $\mathbf{z}_{c}$ é dada por

$$
\begin{aligned}
\ell_{c}\left(\boldsymbol{\theta} \mid \mathbf{z}_{c}\right)= & -\sum_{i=1}^{p} n_{i} \log \sigma_{\epsilon i}^{2}-\frac{N}{2} \log \sigma^{2}-\frac{1}{2} \sum_{i=1}^{p} \sum_{j=1}^{n_{i}}\left(\mathbf{z}_{i j}-x_{i j} \boldsymbol{\beta}_{o i}\right)^{\top} D^{-1}\left(\boldsymbol{\phi}_{i}\right) \\
& \times\left(\mathbf{z}_{i j}-x_{i j} \boldsymbol{\beta}_{o i}\right)-\frac{N}{2} \log \nu^{2}-\frac{1}{2 \nu^{2}} \sum_{i=1}^{p} \sum_{j=1}^{n_{i}}\left(x_{i j}-\mu_{x}-\varsigma t_{i j}\right)^{2} \\
& -\frac{1}{2} \sum_{i=1}^{p} \sum_{j=1}^{n_{i}} t_{i j}^{2}+C,
\end{aligned}
$$

em que $N=\sum_{i=1}^{m} n_{i}$ e $C$ é uma constante que independe do vetor de parâmetros $\theta$. Assim, o algoritmo EM pode ser usado para obter estimativas de máxima verossimilhança de $\boldsymbol{\theta}$ no modelo definido em (4.11).

Passo-E: Dado $\boldsymbol{\theta}=\widehat{\boldsymbol{\theta}}$. Sejam $\widehat{x}_{i j}=E\left[x_{i j} \mid \boldsymbol{\theta}=\widehat{\boldsymbol{\theta}}, \mathrm{z}_{i j}\right], \widehat{x_{i j}^{2}}=E\left[x_{i j}^{2} \mid \boldsymbol{\theta}=\widehat{\boldsymbol{\theta}}, \mathbf{z}_{i j}\right]$, $\widehat{t}_{i j}=E\left[T_{i j} \mid \boldsymbol{\theta}=\widehat{\boldsymbol{\theta}}, \mathbf{z}_{i j}\right], \hat{t}_{i j}^{2}=E\left[T_{i j}^{2} \mid \boldsymbol{\theta}=\widehat{\boldsymbol{\theta}}, \mathbf{z}_{i j}\right]$ e $\widehat{t x_{i j}}=E\left[T_{i j} x_{i j} \mid \boldsymbol{\theta}=\widehat{\boldsymbol{\theta}}, \mathbf{z}_{i j}\right]$, calculamos $\widehat{t}_{i j}, t_{i j}^{2}, \widehat{x}_{i j}, \widehat{x}^{2}{ }_{i j}$ e $\widehat{t x}_{i j}$ para $i=1, \ldots, p$ e $j=1, \ldots, n_{i}$, de forma similar ao modelo 
MGSN (ver Apêndice C), temos que

$$
\begin{aligned}
\widehat{t}_{i j} & =\widehat{\mu}_{T_{i j}}+W_{\Phi_{1}}\left(\frac{\widehat{\mu}_{T_{i j}}}{\widehat{M}_{T_{i}}}\right) \widehat{M}_{T_{i}}, \\
\widehat{t}_{i j}^{2} & =\widehat{\mu}_{T_{i j}}^{2}+\widehat{M}_{T_{i}}^{2}+W_{\Phi_{1}}\left(\frac{\widehat{\mu}_{T_{i j}}}{\widehat{M}_{T_{i}}}\right) \widehat{M}_{T_{i}} \widehat{\mu}_{T_{i j}}, \\
\widehat{x}_{i j} & =\widehat{r}_{i j}+\widehat{s}_{i} \widehat{t}_{i j} \\
\widehat{x_{i j}^{2}} & =\widehat{T}_{x_{i}}^{2}+\widehat{r}_{i j}^{2}+2 \widehat{r}_{i j} \widehat{s}_{i} \widehat{t}_{i j}+\widehat{s}_{i}^{2} \widehat{t}^{2}{ }_{i j} \\
\widehat{t x}_{i j} & =\widehat{r}_{i j} \widehat{t}_{i j}+\widehat{s}_{i} \widehat{t}_{i j},
\end{aligned}
$$

em que $\widehat{M}_{T_{i}}^{2}=\left[1+\widehat{\varsigma}^{2} \widehat{\boldsymbol{\beta}}_{o i}^{\top}\left(D\left(\widehat{\boldsymbol{\phi}}_{i}\right)+\widehat{\nu}^{2} \widehat{\boldsymbol{\beta}}_{o i} \widehat{\boldsymbol{\beta}}_{o i}^{\top}\right)^{-1} \widehat{\boldsymbol{\beta}}_{o i}\right]^{-1}, \quad \widehat{\mu}_{T_{i j}}=\widehat{\varsigma} \widehat{M}_{T_{i}}^{2} \widehat{\boldsymbol{\beta}}_{o i}^{\top}\left(D\left(\widehat{\boldsymbol{\phi}}_{i}\right)+\right.$ $\left.\widehat{\nu}^{2} \widehat{\boldsymbol{\beta}}_{o i} \widehat{\boldsymbol{\beta}}_{o i}^{\top}\right)^{-1}\left(\mathbf{z}_{i j}-\widehat{\boldsymbol{\beta}}_{o i} \widehat{\mu}_{x}\right), \widehat{T}_{x_{i}}^{2}=\widehat{\nu}^{2}\left[1+\widehat{\nu}^{2} \widehat{\boldsymbol{\beta}}_{o i}^{\top} D^{-1}\left(\widehat{\boldsymbol{\phi}}_{i}\right) \widehat{\boldsymbol{\beta}}_{o i}\right]^{-1}, \widehat{r}_{i j}=\widehat{\mu}_{x}+\widehat{T}_{x_{i}}^{2} \widehat{\boldsymbol{\beta}}_{o i}^{\top} D^{-1}\left(\widehat{\phi}_{i}\right)\left(\mathbf{z}_{i j}\right.$ $\left.-\widehat{\boldsymbol{\beta}}_{o i} \widehat{\mu}_{x}\right)$ e $\widehat{s}_{i}=\widehat{\varsigma}\left(1-\widehat{T}_{x_{i}}^{2} \widehat{\boldsymbol{\beta}}_{o i}^{\top} D^{-1}\left(\widehat{\phi}_{i}\right) \widehat{\boldsymbol{\beta}}_{o i}\right)$.

Conseqüentemente, a esperança condicional correspondente a função $Q(\boldsymbol{\theta} \mid \widehat{\boldsymbol{\theta}})$, é expressa por

$$
\begin{aligned}
Q(\boldsymbol{\theta} \mid \widehat{\boldsymbol{\theta}})= & E\left[\ell_{c}\left(\boldsymbol{\theta} \mid \mathbf{z}_{c}\right) \mid \mathbf{z}, \widehat{\boldsymbol{\theta}}\right]=-\sum_{i=1}^{p} n_{i} \log \sigma_{\epsilon i}^{2}-\frac{N}{2} \log \sigma^{2} \\
& -\frac{1}{2} \sum_{i=1}^{p} \sum_{j=1}^{n_{i}}\left(\mathbf{z}_{i j}-\boldsymbol{\beta}_{o i} \widehat{x}_{i j}\right)^{\top} D^{-1}\left(\phi_{i}\right)\left(\mathbf{z}_{i j}-\boldsymbol{\beta}_{o i} \widehat{x}_{i j}\right) \\
& \left.-\frac{1}{2} \sum_{i=1}^{p} \sum_{j=1}^{n_{i}} \widehat{\left(x_{i j}^{2}\right.}-\left(\widehat{x}_{i j}\right)^{2}\right) \boldsymbol{\beta}_{o i}^{\top} \mathrm{D}^{-1}(\phi) \boldsymbol{\beta}_{o i}-\frac{N}{2} \log \left(\nu^{2}\right) \\
& -\frac{1}{2 \nu^{2}} \sum_{i=1}^{p} \sum_{j=1}^{n_{i}}\left(\widehat{x_{i j}^{2}}+\mu_{x}^{2}+\varsigma^{2} \widehat{t_{i j}^{2}}-2 \widehat{x}_{i j} \mu_{x}-2 \varsigma \widehat{t x_{i j}}+2 \varsigma \mu_{x} \widehat{t}_{i j}\right) \\
& -\frac{1}{2} \sum_{i=1}^{p} \sum_{j=1}^{n_{i}} \widehat{t_{i j}^{2}}+C,
\end{aligned}
$$


Passo-M: Dado $\widehat{\boldsymbol{\theta}}$, maximizamos $Q(\boldsymbol{\theta} \mid \widehat{\boldsymbol{\theta}})$ com respeito a $\boldsymbol{\theta}$, de (4.16) temos como resultados

$$
\begin{aligned}
\widehat{\beta}_{k i} & =\frac{\sum_{j=1}^{n_{i}} y_{k i j} \widehat{x}_{i j}}{\sum_{j=1}^{n_{i}}{\widehat{x^{2}}}_{i j}}, k=1,2, i=1, \ldots, p \\
\widehat{\mu}_{x} & =\frac{1}{N} \sum_{i=1}^{p} \sum_{j=1}^{n_{i}}\left(\widehat{x}_{i j}-\varsigma \widehat{t}_{i j}\right), \\
\widehat{\sigma}_{\epsilon i}^{2} & =\frac{1}{2 n_{i}} \sum_{j=1}^{n_{i}}\left(y_{1 i j}^{2}+y_{2 i j}^{2}-2 \widehat{x}_{i j}\left(y_{1 i j} \beta_{1 i}+y_{2 i j} \beta_{2 i}\right)+\widehat{x}^{2}{ }_{i j}\left(\beta_{1 i}^{2}+\beta_{2 i}^{2}\right)\right) \\
\widehat{\sigma}^{2} & =\frac{1}{N} \sum_{i=1}^{p} \sum_{j=1}^{n_{i}}\left(x_{i j}^{2}-2 \widehat{x}_{i j} x_{i j}+\widehat{x_{i j}^{2}}\right), \widehat{\varsigma}=\frac{\sum_{i=1}^{p} \sum_{j=1}^{n_{i}}\left(\widehat{t x_{i j}}-\mu_{x} \widehat{t}_{i j}\right)}{\sum_{i=1}^{p} \sum_{j=1}^{n_{i}} \widehat{t}_{i j}^{2}} \\
\widehat{\nu}^{2} & =\frac{1}{N} \sum_{i=1}^{p} \sum_{j=1}^{n_{i}}\left(\widehat{x}^{2}{ }_{i j}+\mu_{x}^{2}+\varsigma^{2} \widehat{t}_{i j}^{2}-2 \mu_{x} \widehat{x}_{i j}-2 \varsigma \widehat{t x_{i j}}+2 \varsigma \mu_{x} \widehat{t}_{i j}\right) .
\end{aligned}
$$

Note que $\widehat{\beta_{k i}}$ pode ser escrita, alternativamente, como $\widehat{\beta}=\Delta_{x}^{-1} \eta_{x y}$, onde $\Delta_{x}=$ $\operatorname{diag}\left(\sum_{j=1}^{n_{1}} \widehat{x_{1 j}^{2}}, \ldots, \sum_{j=1}^{n_{p}} \widehat{x_{p j}^{2}}, \sum_{j=1}^{n_{1}} \widehat{x_{1 j}^{2}}, \ldots, \sum_{j=1}^{n_{p}} \widehat{x_{p j}^{2}}\right)$ e $\eta_{x y}=\left(\sum_{j=1}^{n_{1}} y_{11 j} \widehat{x}_{1 j}, \ldots\right.$, $\left.\sum_{j=1}^{n_{p}} y_{1 p j} \widehat{x}_{p j}, \sum_{j=1}^{n_{1}} y_{21} \widehat{x}_{1 j}, \sum_{j=1}^{n_{1}} \ldots, \sum_{j=1}^{n_{p}} y_{2 p j} \widehat{x}_{p j}\right)^{\top}$. Os parâmetros de forma escala da variável latente $\mathbf{x}$, podem ser estimados notando que $\varsigma / \nu=\lambda_{x}$, e $\phi_{x}=\varsigma^{2}+\nu^{2}$. Os valores iniciais são freqüentemente escolhidos como sendo as estimativas correspondentes ao modelo normal (MINMN), sendo que o valor inicial para o parâmetro de assimetria é igual a zero.

\subsubsection{Influência local}

Definições e propriedades sobre influência local apresentadas na Seção 2.5 são utilizadas nesta seção para o modelo de intercepto nulo com erro de medida normalassimétrico para uma população. 


\subsection{Esquemas de perturbação para o MINSN}

Nesta seção, obtemos a matriz $\Delta$ definida em (2.23)para diferentes esquemas de perturbação.

\subsubsection{Perturbação da Ponderação de Casos}

O logaritmo da função de verossimilhança para o modelo (4.3) e (4.4) é dado por (4.9) em que $\ell_{i j}(\theta)$, é a contribuição da $i j$-ésima observação (pesos iguais) para a verossimilhança, $i=1, \ldots, p, j=1, \ldots, n_{i}$. A função de log-verossimilhança perturbada permite diferentes pesos para diferentes observações, pode ser definida por

$$
\ell(\boldsymbol{\theta} \mid \boldsymbol{\omega})=\sum_{i=1}^{p} \sum_{j=1}^{n_{i}} \omega_{i j} \ell_{i j}(\boldsymbol{\theta}),
$$

em que, $\boldsymbol{\theta}=\left(\mu_{x}, \beta_{1}, \ldots, \beta_{p}, \phi_{x}, \sigma_{1}^{2}, \ldots, \sigma_{p}^{2}, \sigma_{u}^{2}, \lambda_{x}\right)^{\top}, \boldsymbol{\omega}=\left(\omega_{11}, \ldots, \omega_{1 n_{1}}, \ldots, \omega_{p 1}, \ldots\right.$, $\left.\omega_{p n_{p}}\right)^{\top}$ é o vetor de pesos da contribuição de cada observação da verossimilhança e $\omega_{0}=1_{N}=(1, \ldots, 1)^{\top}$, com $N=\sum_{i=1}^{p} n_{i}$, é o ponto de ausência de perturbação, isto é, $l\left(\theta \mid \omega_{0}\right)=l(\theta)$. Este esquema de perturbação pretende avaliar se a contribuição das observações com diferentes pesos afeta o estimador de máxima verossimilhança $\boldsymbol{\theta}$. Logo, usando (4.17) e depois de algumas manipulações algébricas, a matriz delta é dada por

$$
\Delta=\left(\Delta_{11}(\theta), \ldots, \Delta_{1 n_{1}}(\theta), \ldots, \Delta_{p 1}(\theta), \ldots, \Delta_{p n_{p}}(\theta)\right)
$$

em que $\Delta_{i j}=\frac{\partial \ell_{i j}(\boldsymbol{\theta})}{\partial \boldsymbol{\theta}}, i=1, \ldots, p, j=1, \ldots, n_{i}$, cujos elementos individuais são dados por

$$
\frac{\partial l_{i j}(\boldsymbol{\theta})}{\partial \gamma}=-\frac{1}{2} \frac{\partial \log \left|\Sigma_{i}\right|}{\partial \gamma}-\frac{1}{2} \frac{\partial g_{i j}}{\partial \gamma}+\frac{\partial \log \left(K_{i j}\right)}{\partial \gamma}, \gamma=\mu_{x}, \beta_{1}, \ldots, \beta_{p}, \phi_{x}, \lambda_{x}, \sigma_{1}^{2}, \ldots, \sigma_{p}^{2}, \sigma_{u}^{2} .
$$


Os componentes de $\frac{\partial \log \left|\Sigma_{i}\right|}{\partial \gamma}, \frac{\partial g_{i j}}{\partial \gamma}$ e $\frac{\partial \log \left(K_{i j}\right)}{\partial \gamma}$ são apresentados em (4.10) (ver também Apêndice D.2). A matriz $\Delta$ apresentada acima deve ser avaliada nos EMV's.

\subsubsection{Perturbação na variável resposta}

Nesta seção nosso interesse é detectar a sensibilidade do modelo quando submetemos a variável resposta, ou seja, a uma perturbação aditiva, dado por

$$
Y_{i j}\left(\omega_{i j}\right)=Y_{i j}+S_{i} \omega_{i j}
$$

em que $S_{i}$ é um fator de escala, por exemplo, podemos considerar como o desvio padrão amostral correspondente a cada grupo de indivíduos, $i=1, \ldots, p$, e $\boldsymbol{\omega}=$ $\left(\omega_{11}, \ldots, \omega_{1 n_{1}}, \ldots, \omega_{p 1} \ldots \omega_{p n_{p}}\right)^{\top}$. O modelo não perturbado é obtido tomarmos $\omega_{o}=$ 0. A função de log-verossimilhança perturbada segue de (4.9) com $y_{i j}\left(\omega_{i j}\right)$ substituído por $y_{i j}, i=1, \ldots, p, j=1, \ldots, n_{i}$. Isto é,

$$
\ell(\boldsymbol{\theta} \mid \boldsymbol{\omega})=\sum_{i=1}^{p} \sum_{j=1}^{n_{i}} \ell_{i j}\left(\boldsymbol{\theta} \mid \omega_{i j}\right),
$$

em que $\ell_{i j}\left(\boldsymbol{\theta} \mid \omega_{i j}\right)=-\frac{1}{2} \log \left|\Sigma_{i}\right|-\frac{1}{2} g_{i j}\left(\omega_{i j}\right)+\log \left(K_{i j}\left(\omega_{i j}\right)\right)+C \operatorname{com} g_{i j}\left(\omega_{i j}\right)=$ $\left(\mathbf{z}_{i j}\left(\omega_{i j}\right)-\boldsymbol{\mu}_{i}\right)^{\top} \Sigma_{i}^{-1}\left(\mathbf{z}_{i j}\left(\omega_{i j}\right)-\boldsymbol{\mu}_{i}\right), K_{i j}\left(\omega_{i j}\right)=\Phi_{1}\left(A_{i} a_{i j}\left(\omega_{i j}\right)\right)$ e $a_{i j}\left(\omega_{i j}\right)=\left(\mathbf{z}_{i j}\left(\omega_{i j}\right)-\right.$ $\left.\mu_{i}\right)^{\top} D^{-1}\left(\phi_{i}\right) \boldsymbol{\beta}_{0 i}$.

Derivando $\ell(\boldsymbol{\theta} \mid \boldsymbol{\omega})$ em relação a $\boldsymbol{\omega}$ e $\boldsymbol{\theta}$ temos que a matriz $\Delta$ é dada por

$$
\Delta=\left(\Delta_{11}(\theta), \ldots, \Delta_{1 n_{1}}(\theta), \ldots, \Delta_{p 1}(\theta), \ldots, \Delta_{p n_{p}}(\theta)\right),
$$

em que

$$
\begin{aligned}
\Delta_{i j}(\boldsymbol{\theta})= & \frac{\partial P_{i j}\left(\omega_{i j}\right)}{\partial \boldsymbol{\theta}}+W_{\Phi_{1}}\left(A_{i} a_{i j}\left(\omega_{i j}\right)\right)\left\{\frac{\partial A_{i}}{\partial \boldsymbol{\theta}} Q_{i j}\left(\omega_{i j}\right)+A_{i} \frac{\partial Q_{i j}\left(\omega_{i j}\right)}{\partial \boldsymbol{\theta}}\right\} \\
& +A_{i} W_{\Phi_{1}}^{\prime}\left(A_{i} a_{i j}\left(\omega_{j}\right)\right) Q_{i j}\left(\omega_{i j}\right)\left\{A_{i} \frac{\partial a_{i j}\left(\omega_{i j}\right)}{\partial \boldsymbol{\theta}}+a_{i j}\left(\omega_{i j}\right) \frac{\partial A_{i}}{\partial \boldsymbol{\theta}}\right\}
\end{aligned}
$$


$\operatorname{com} P_{i j}\left(\omega_{i j}\right)=-\left(\mathbf{z}_{i j}\left(\omega_{i j}\right)-\boldsymbol{\mu}_{i}\right)^{\top} \Sigma_{i}^{-1} \frac{\partial \mathbf{z}_{i j}\left(\omega_{i j}\right)}{\partial \omega_{i j}}, Q_{i j}\left(\omega_{i j}\right)=\frac{\partial \mathbf{z}_{i j}\left(\omega_{i j}\right)^{\top}}{\partial \omega_{i j}} D^{-1}\left(\phi_{i}\right) \boldsymbol{\beta}_{0 i}$ e $\frac{\partial a_{i j}\left(\omega_{i j}\right)}{\partial \boldsymbol{\theta}}$ é calculada da mesma forma que no modelo não perturbado (Apêndice D.2), substituímos $\mathbf{z}_{i j}=\left(X_{i j}, y_{i j}\right)^{\top}$ por $\mathbf{z}_{i j}\left(\omega_{i j}\right)=\left(X_{i j}, y_{i j}+S_{i} \omega_{i j}\right)^{\top}, i=1, \ldots, p, j=$ $1, \ldots, n_{i}$. Extensivas manipulações algébricas nos leva às expressões dessas derivadas, apresentadas no Apêndice F.3.

\subsubsection{Perturbação na variável explanatória}

Se estamos interesados em avaliar a sensibilidade de pequenas perturbações na variável explanatória sobre as estimativas de máxima verossimilhança, definimos o seguinte esquema de perturbação para a variável explanatória da mesma forma como foi definido na Seção 4.4.2 para a variável resposta. Seja

$$
X_{i j}\left(\omega_{j}\right)=X_{i j}+S_{i} \omega_{i j}
$$

em que $S_{i}$ é definido como na subseção anterior e $\omega=\left(\omega_{11}, \ldots, \omega_{1 n_{1}}, \ldots, \omega_{p 1} \ldots \omega_{p n_{p}}\right)^{\top}$. Para o caso não perturbado segue por considerar $\omega_{o}=\mathbf{0}$. A função de log-verossimilhança perturbada segue de (4.9) com $X_{i j}\left(\omega_{i j}\right)$ substituindo por $X_{i j}, i=1, \ldots, p, j=$ $1, \ldots, n_{i}$. Como na perturbação de variável resposta, a matriz $\Delta$ é como dada em (4.18), com

$$
\begin{gathered}
\Delta_{i j}(\boldsymbol{\theta})=\frac{\partial R_{i j}\left(\omega_{i j}\right)}{\partial \boldsymbol{\theta}}+W_{\Phi_{1}}\left(A_{i} a_{i j}\left(\omega_{i j}\right)\right)\left\{\frac{\partial A_{i}}{\partial \boldsymbol{\theta}} V_{i j}\left(\omega_{i j}\right)+A_{i} \frac{\partial V_{i j}\left(\omega_{i j}\right)}{\partial \boldsymbol{\theta}}\right\} \\
+A_{i} W_{\Phi_{1}}^{\prime}\left(A_{i} a_{i j}\left(\omega_{i j}\right)\right) V_{i j}\left(\omega_{i j}\right)\left\{A_{i} \frac{\partial a_{i j}\left(\omega_{i j}\right)}{\partial \boldsymbol{\theta}}+a_{i j}\left(\omega_{i j}\right) \frac{\partial A_{i}}{\partial \boldsymbol{\theta}}\right\}, \\
\operatorname{com~} R_{i j}\left(\omega_{i j}\right)=-\left(\mathbf{z}_{i j}\left(\omega_{i j}\right)-\boldsymbol{\mu}_{i}\right)^{\top} \Sigma_{i}^{-1} \frac{\partial \mathbf{z}_{i j}\left(\omega_{i j}\right)}{\partial \omega_{i j}}, V_{i j}\left(\omega_{i j}\right)=\frac{\partial \mathbf{z}_{i j}\left(\omega_{i j}\right)}{\partial \omega_{i j}} D^{-1}\left(\phi_{i}\right) \boldsymbol{\beta}_{0 i} \text { e } \\
\frac{\partial a_{i j}\left(\omega_{i j}\right)}{\partial \boldsymbol{\theta}} \text { é calculada como no modelo não perturbado substituindo } \mathbf{z}_{i j}=\left(X_{i j}, y_{i j}\right)^{\top}
\end{gathered}
$$


por $\mathbf{z}_{i j}\left(\omega_{i j}\right)=\left(X_{i j}+S_{i} \omega_{i j}, y_{i j}\right)^{\top}$. Expressões destas derivadas são apresentadas no Apêndice F.4.

\subsection{Aplicação}

Nesta seção ilustramos a utilidade da metodologia proposta aplicando-a a uma parte do conjunto de dados reais analisado em Hadgu e Koch (1999) usando equações de estimação generalizada, assumindo distribuição gama para a variável resposta. Deste estudo participaram 109 voluntários adultos do sexo masculino com placa dental préexistente aleatorizados para uso de dois enxágües bucais (A e B) e o enxágüe controle (C) que pode representar simplesmente água. Foram avaliados os índices de placa dentária no início do estudo, depois de três e depois de seis meses. Com o intuito de utilizar o MINSN definido em (4.3)-(4.4), utilizamos apenas os resultados de medidas tomadas após três meses, apresentados na Tabela 4.2, Observe que como a covariável (índice de placa dentária) está sujeita a erro de medição, uma forma alternativa para analizar este conjunto de dados é considerado um modelo com erro de medida. Note também, que se o índice de placa dentária no início do estudo for nulo, é de se esperar que depois o índice seja nulo, isto é, o índice de placa dentária não deveria ter aumento depois do uso de cada enxágüe bucal.

Resultados das estimativas dos parâmetros dos modelos MINSN e MINN são apresentados na Tabela 4.1. Estimativas dos parâmetros são obtidas de forma fechada em ambos modelos, excepto para as estimativas de $\mu_{x}$ e $\sigma_{x}^{2}$. Além disso, parece haver uma indicação significativa da redução do índice da placa dentária. Note que o desvio padrão estimado para $\lambda_{x}$ parece ser grande, mas os valores de AIC, BIC e HQ apresentados na parte inferior da Tabela 4.1 parecem favorecer o MINSN do que 
MINN, sustentando a suposição de não-normalidade. Uma conclusão mais enfática nessa direção pode ser vista considerando um teste paramétrico para normalidade, onde a hipótese nula é $\lambda_{x}=0$, tal como o teste da razão de verossimilhanças, para o qual o valor observado da estatística de teste é 18,23 e o nível crítico associado da distribuição $\chi^{2}(1)$ em $5 \%$ é 3,84 . Esta conclusão também é enfatizada pelo gráfico PP-plot (Azzalini e Capitanio, 1999) mostrado na Figura 4.1 (a), usando resultados em (4.8).

Figura 4.1: Conjunto de dados de índice de placa dentária. (a) Gráfico PP de Healy do MINSN (linha contínua) e MINN (linha tracejada). (b) Histograma da covariável observada X (índice de placa no início do estudo) sobreposto pelas densidades estimadas das distribuições normal-assimétrica (linha contínua) e normal (linha tracejada).

(a)

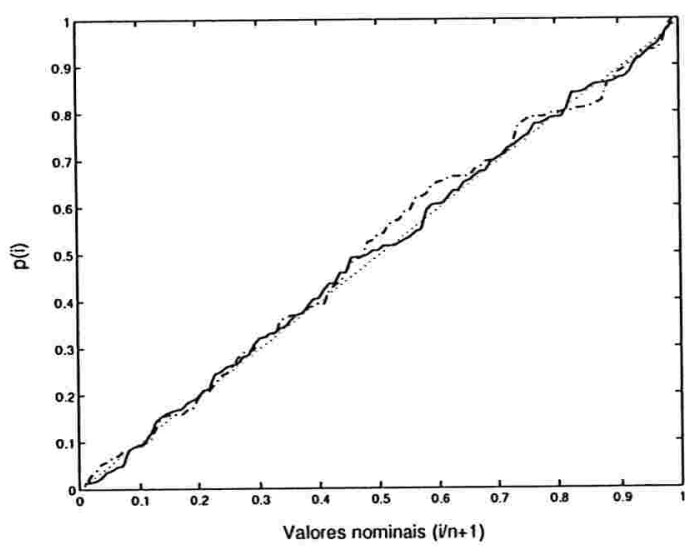

(b)

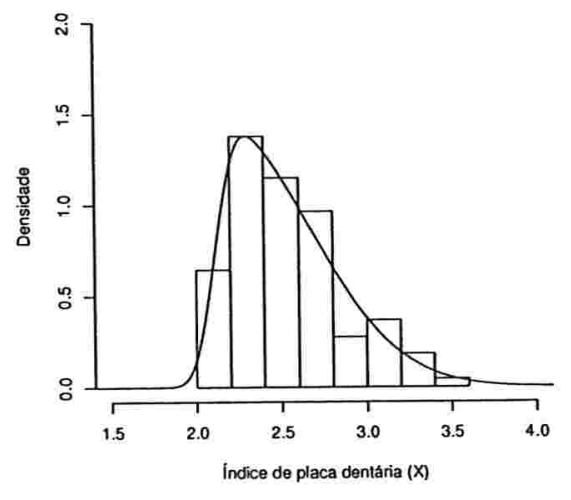

Na Figura 4.2 apresentamos os gráficos de índice de $\left|d_{\max }\right|$ e $C_{j}$ sob o esquema de perturbação da ponderação de casos. Baseado neste esquema encontramos que os indivíduos 27 do enxágüe bucal controle (C), 26 (referente ao indivíduo 99 de B) e 2, 3 e 6 (referentes aos indivíduos 42,43 e 46 de A) são os mais influentes sobre $\widehat{\theta}$ 
sob o MINSN. Este resultado discorda com alguns encontrados sob o MINN em que os indivíduos 31 de B e 6 e 16 do grupo A são os mais influentes. Com o intuito de comparar com os resultados de influência global, na Figura 4.3 apresentamos os gráficos de índice do afastamento da verossimilhança $\left(L D_{j}\right)$ e a distância de Cook $\left(D_{j}\right), j=1, \ldots, N$. Note que, $D_{j}$ mostra os indivíduos 2,3 e 6 de A como os mais influentes globalmente, como descrito também na Figura 4.2 (b) usando influência local total $C_{j}$.

Note que para a perturbação da variável resposta e da variável explanatória encontramos $C_{d \max }(\widehat{\boldsymbol{\theta}})=9.335$ e $C_{d \max }(\widehat{\boldsymbol{\theta}})=97.760$, respectivamente. O gráfico de índice $\left|\ell_{\max }\right|$ sob estes os esquemas de perturbação é mostrado na Figura 4.4. Quando o esquema de perturbação da variável resposta é considerado, a Figura 4.4 (a) mostra que o enxágüe bucal A é potencialmente influente, com os indivíduos 2, 3 e 6 como sendo os mais destacados. Sob a perturbação da variável explanatória, os indivíduos 21 e 27 de enxágüe bucal controle são os mais influentes e o resultado é expressivamente diferente do modelo MINN. 
Tabela 4.1: Resultado do ajuste do modelo de intercepto nulo com erro de medida normal-assimétrico e normal (MINSN e MINN) para o conjunto de dados de índice de placa dentária. EP são erros padrão estimados assintóticamente baseados na matriz de informação.

\begin{tabular}{|c|c|c|c|c|}
\hline \multirow[b]{2}{*}{ Parâmetros } & \multicolumn{2}{|c|}{ MINSN } & \multicolumn{2}{|l|}{ MINN } \\
\hline & Estimativas & $\mathrm{EP}$ & Estimativas & $\mathrm{EP}$ \\
\hline$\beta_{1}$ & 0.7100 & 0.0341 & 0.7100 & 0.0341 \\
\hline$\beta_{2}$ & 0.5282 & 0.0435 & 0.5281 & 0.0469 \\
\hline$\beta_{3}$ & 0.5088 & 0.0339 & 0.5088 & 0.0339 \\
\hline$\sigma_{1}^{2}$ & 0.3009 & 0.0714 & 0.3006 & 0.0724 \\
\hline$\sigma_{2}^{2}$ & 0.4282 & 0.1042 & 0.4978 & 0.1423 \\
\hline$\sigma_{3}^{2}$ & 0.2563 & 0.0605 & 0.2562 & 0.0606 \\
\hline$\sigma_{u}^{2}$ & 0.0010 & 0.0271 & 0.0010 & 0.0399 \\
\hline$\mu_{x}$ & 2.1127 & 0.0405 & 2.5367 & 0.0317 \\
\hline$\sigma_{x}^{2}$ & 0.2879 & 0.0558 & 0.1082 & 0.0357 \\
\hline$\lambda_{x}$ & 6.0843 & 5.4569 & - & - \\
\hline -log-verossimilhança & \multicolumn{2}{|c|}{-7.4760} & \multicolumn{2}{|c|}{-1.6454} \\
\hline AIC & \multicolumn{2}{|c|}{2.5240} & \multicolumn{2}{|c|}{10.6454} \\
\hline $\mathrm{BIC}$ & \multicolumn{2}{|c|}{15.9808} & \multicolumn{2}{|c|}{22.7565} \\
\hline $\mathrm{HQ}$ & \multicolumn{2}{|c|}{7.9812} & \multicolumn{2}{|c|}{15.5569} \\
\hline
\end{tabular}


Figura 4.2: Conjunto de dados de índice de placa dentária, interesse em $\boldsymbol{\theta}$. Gráfico de índice: (a) $|d \max |$ e (b) $C_{j}$ para a perturbação da ponderação de casos. (-) e (...) denotando o gráfico de índice para MINSN e MINN, respectivamente.

(a)

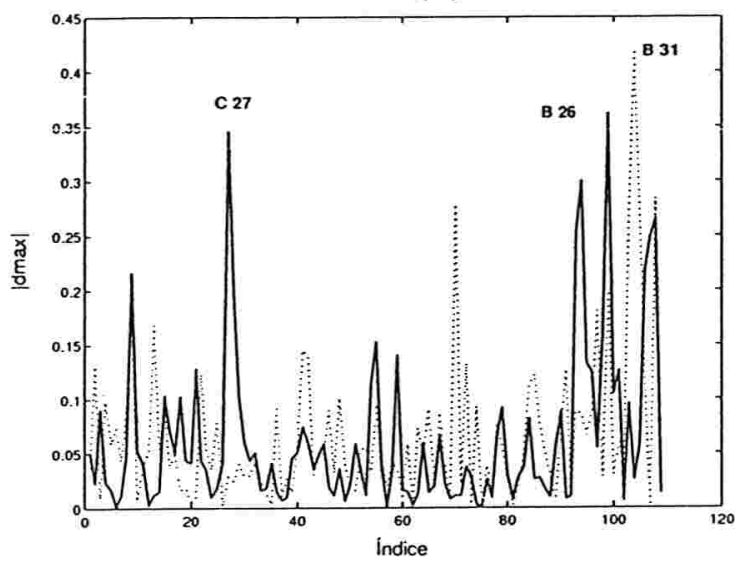

(b)

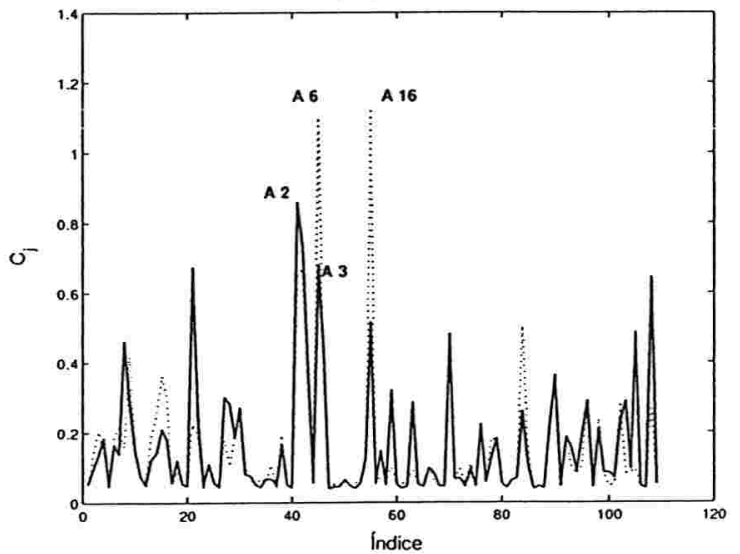

Figura 4.3: Conjunto de dados de índice de placa dentária, modelo MINSN. Gráfico de índice: (a) Afastamento da verossimilhança, $L D_{j}$ e (b) distância de Cook, $D_{j}$.

(a)

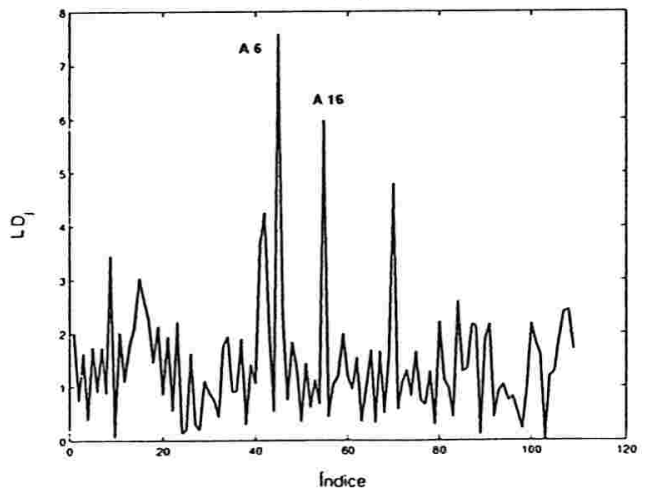

(b)

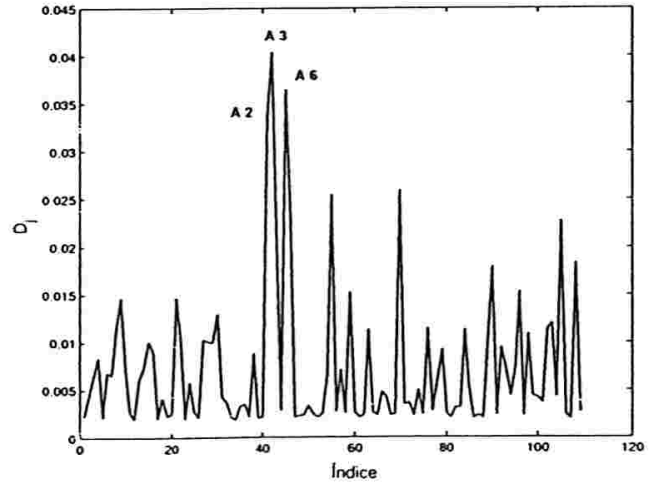


Figura 4.4: Conjunto de dados de índice de placa dentária, interesse em $\boldsymbol{\theta}$. Gráfico de índice: (a) $|d \max |$ para perturbação da variável resposta e (b) |dmax $\mid$ para perturbação da variável explanatória. (-) e (...) denotando o gráfico de índice para os MINSN e MINN, respectivamente.

(a)

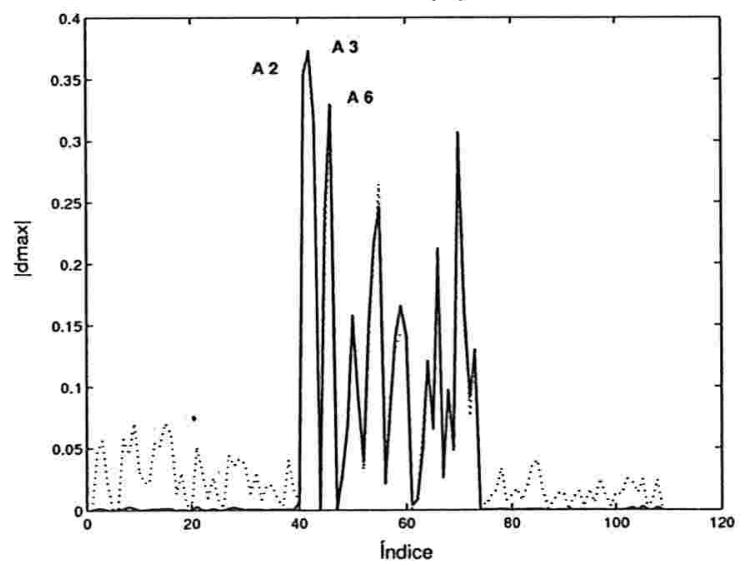

(b)

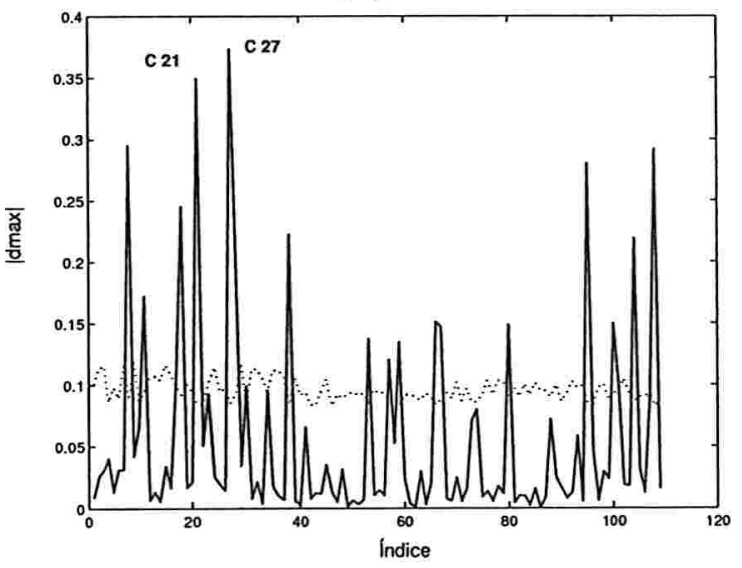


Tabela 4.2: Conjunto de dados referente a enxágüe bucal.

\begin{tabular}{|c|c|c|c|c|c|c|c|c|c|c|c|}
\hline OBS. & SEX & IDADE & $T M T$ & $\begin{array}{c}P I N D E X 0 \\
(\mathrm{X})\end{array}$ & $\begin{array}{c}\text { PINDEX3 } \\
(Y)\end{array}$ & OBS. & SEX & IDADE & $T M T$ & $\begin{array}{c}\text { PINDEXO } \\
(\mathrm{X})\end{array}$ & $\begin{array}{c}P I N D E X 3 \\
(\mathrm{Y})\end{array}$ \\
\hline 1 & 2 & 24 & $\mathrm{C}$ & 2.46 & 1.73 & 56 & 1 & 25 & A & 2.96 & 1.48 \\
\hline 2 & 2 & 29 & C & 2.89 & 2.61 & 57 & 2 & 30 & A & 2.16 & 1.46 \\
\hline 3 & 1 & 27 & C & 3.00 & 2.75 & 58 & 1 & 28 & A & 2.34 & 0.70 \\
\hline 4 & 2 & 25 & $\mathrm{C}$ & 2.52 & 0.98 & 59 & 1 & 25 & A & 2.04 & 0.46 \\
\hline 5 & 1 & 27 & C & 2.52 & 1.61 & 60 & 2 & 25 & A & 2.35 & 1.78 \\
\hline 6 & 2 & 25 & C & 2.78 & 1.25 & 61 & 2 & 25 & A & 2.68 & 1.43 \\
\hline 7 & 2 & 27 & C & 3.02 & 2.76 & 62 & 2 & 29 & A & 2.50 & 1.35 \\
\hline 8 & 1 & 25 & C & 2.08 & 0.88 & 63 & 2 & 43 & A & 2.07 & 1.29 \\
\hline 9 & 1 & 29 & $\mathrm{C}$ & 3.11 & 3.04 & 64 & 2 & 36 & A & 2.52 & 1.79 \\
\hline 10 & 1 & 28 & C & 2.38 & 0.90 & 65 & 2 & 26 & A & 2.37 & 1.50 \\
\hline 11 & 1 & 28 & C & 2.24 & 1.37 & 66 & 1 & 25 & A & 2.27 & 0.39 \\
\hline 12 & 1 & 24 & C & 2.70 & 2.11 & 67 & 2 & 26 & A & 2.21 & 1.27 \\
\hline 13 & 2 & 38 & C & 3.20 & 2.39 & 68 & 2 & 23 & A & 2.71 & 1.80 \\
\hline 14 & 2 & 36 & C & 3.25 & 2.23 & 69 & 2 & 40 & A & 2.45 & 1.10 \\
\hline 15 & 1 & 27 & C & 3.21 & 2.88 & 70 & 1 & 25 & A & 3.11 & 2.81 \\
\hline 16 & 2 & 23 & C & 3.30 & 2.50 & 71 & 2 & 27 & A & 2.52 & 0.71 \\
\hline 17 & 2 & 27 & C & 2.27 & 1.61 & 72 & 2 & 22 & A & 3.02 & 1.94 \\
\hline 18 & 1 & 25 & C & 2.21 & 1.15 & 73 & 1 & 26 & A & 2.32 & 0.73 \\
\hline 19 & 1 & 25 & C & 2.40 & 1.69 & 74 & 2 & 23 & B & 2.33 & 0.56 \\
\hline 20 & 1 & 29 & C & 2.64 & 1.45 & 75 & 2 & 38 & B & 2.53 & 1.00 \\
\hline 21 & 1 & 24 & C & 2.07 & 0.70 & 76 & 1 & 24 & B & 2.61 & 2.11 \\
\hline 22 & 1 & 28 & C & 2.43 & 0.85 & 77 & 2 & 47 & B & 2.74 & 1.02 \\
\hline 23 & 1 & 29 & C & 2.27 & 1.71 & 78 & 1 & 23 & B & 3.02 & 2.04 \\
\hline 24 & 1 & 23 & C & 2.52 & 2.50 & 79 & 2 & 24 & B & 2.56 & 2.04 \\
\hline 25 & 1 & 28 & C & 2.79 & 1.54 & 80 & 2 & 25 & B & 2.25 & 0.96 \\
\hline 26 & 1 & 29 & C & 2.54 & 1.58 & 81 & 1 & 28 & B & 2.74 & 1.54 \\
\hline 27 & 1 & 23 & C & 2.17 & 0.77 & 82 & 1 & 25 & B & 2.60 & 0.88 \\
\hline 28 & 1 & 26 & C & 2.09 & 1.16 & 83 & 1 & 25 & B & 2.76 & 1.82 \\
\hline 29 & 1 & 24 & C & 2.68 & 2.74 & 84 & 1 & 23 & B & 3.11 & 0.93 \\
\hline 30 & 1 & 24 & C & 2.35 & 0.72 & 85 & 1 & 24 & B & 3.13 & 1.85 \\
\hline 31 & 2 & 29 & C & 2.36 & 2.33 & 86 & 2 & 24 & B & 2.71 & 1.36 \\
\hline 32 & 2 & 38 & C & 2.61 & 2.43 & 87 & 2 & 28 & B & 2.42 & 1.36 \\
\hline 33 & 2 & 24 & C & 2.48 & 2.00 & 88 & 1 & 31 & B & 2.32 & 0.92 \\
\hline 34 & 2 & 29 & C & 2.31 & 1.33 & 89 & 2 & 24 & B & 2.19 & 1.85 \\
\hline 35 & 1 & 30 & C & 2.50 & 2.32 & 90 & 1 & 23 & B & 2.72 & 0.50 \\
\hline 36 & 1 & 32 & C & 2.31 & 2.27 & 91 & 1 & 30 & B & 2.45 & 1.20 \\
\hline 37 & 2 & 32 & C & 2.38 & 1.98 & 92 & 2 & 25 & B & 2.60 & 2.06 \\
\hline 38 & 1 & 24 & C & 2.27 & 0.75 & 93 & 1 & 25 & B & 2.17 & 1.60 \\
\hline 39 & 2 & 30 & C & 2.61 & 2.07 & 94 & 2 & 32 & B & 2.38 & 1.78 \\
\hline 40 & 2 & 42 & A & 2.65 & 1.42 & 95 & 2 & 35 & B & 2.18 & 0.55 \\
\hline 41 & 2 & 48 & A & 2.13 & 2.48 & 96 & 2 & 26 & B & 2.38 & 0.33 \\
\hline 42 & 2 & 27 & A & 2.70 & 0.00 & 97 & 1 & 25 & B & 2.88 & 1.53 \\
\hline 43 & 2 & 33 & A & 2.48 & 0.10 & 98 & 2 & 37 & B & 2.21 & 1.90 \\
\hline 44 & 1 & 25 & A & 2.98 & 1.57 & $\theta 9$ & 2 & 29 & B & 2.42 & 0.67 \\
\hline 45 & 2 & 43 & A & 3.57 & 2.80 & 100 & 2 & 35 & B & 2.21 & 1.17 \\
\hline 46 & 2 & 25 & A & 2.48 & 0.05 & 101 & 2 & 31 & B & 2.22 & 1.46 \\
\hline 47 & 2 & 23 & A & 2.73 & 1.44 & 102 & 1 & 24 & B & 2.86 & 2.20 \\
\hline
\end{tabular}




\begin{tabular}{|c|c|c|c|c|c|c|c|c|c|c|c|}
\hline OBS. & SEX & IDADE & $T M T$ & $\begin{array}{c}P I N D E X 0 \\
\text { (X) }\end{array}$ & $\begin{array}{c}\text { PINDEX3 } \\
\text { (Y) }\end{array}$ & OBS. & SEX & IDADE & $T M T$ & $\begin{array}{c}\text { PINDEXO } \\
(\mathrm{X})\end{array}$ & $\begin{array}{c}P I N D E X 3 \\
\text { (Y) }\end{array}$ \\
\hline 48 & 2 & 27 & A & 2.35 & 1.35 & 103 & 2 & 25 & B & 2.06 & 1.13 \\
\hline 49 & 1 & 24 & A & 2.52 & 1.59 & 104 & 1 & 25 & B & 2.23 & 0.61 \\
\hline 50 & 1 & 25 & A & 2.61 & 1.98 & 105 & 1 & 25 & B & 2.02 & 1.00 \\
\hline 51 & 2 & 24 & A & 2.43 & 1.63 & 106 & 2 & 25 & B & 2.45 & 0.98 \\
\hline 52 & 2 & 25 & A & 2.75 & 1.60 & 107 & 1 & 25 & B & 2.29 & 1.13 \\
\hline 53 & 1 & 25 & A & 2.27 & 0.63 & 108 & 2 & 24 & B & 2.06 & 0.27 \\
\hline 54 & 2 & 38 & A & 2.68 & 2.24 & 109 & 1 & 25 & B & 2.44 & 0.88 \\
\hline 55 & 2 & 31 & A & 3.40 & 0.85 & & & & & & \\
\hline
\end{tabular}




\section{Capítulo 5}

\section{Modelo linear misto normal-assi- métrico}

\subsection{Introdução}

No presente capítulo, apresentamos o estudo sobre o modelo linear misto considerando distribuição normal-assimétrica multivariada para os efeitos aleatórios, sendo este modelo uma extensão da versão univariada proposta por Sahu et al. (2003). Para este modelo, implementamos o algoritmo EM para estimar os parâmetros do modelo em estudo, e calculamos as esperanças condicionais relacionadas com os dados completos da função de log-verossimilhança para obter medidas de diagnóstico a partir de uma abordagem de influência local introduzida por Zhu e Lee (2001) sobre quatro esquemas de perturbação. Os resultados obtidos são mostrados através de um conjunto de dados reais. 


\subsection{Especificação do modelo}

Em geral, o modelo linear misto é definido como

$$
\mathrm{Y}_{i}=\mathrm{X}_{i} \boldsymbol{\beta}+\mathrm{Z}_{i} \mathrm{~b}_{i}+\epsilon_{i}, \quad i=1, \ldots, n
$$

em que $\mathrm{Y}_{i}$ de dimensão $\left(n_{i} \times 1\right)$ é um vetor de respostas contínuas observadas em uma unidade amostral $i, \mathbf{X}_{i}$ de dimensão $\left(n_{i} \times p\right)$ é a matriz de planejamento correspondente ao efeito fixo, $\boldsymbol{\beta}$ de dimensão $(p \times 1)$ é um vetor de coeficientes da regressão da média populacional de efeitos fixos, $\mathbf{Z}_{i}$ de dimensão $\left(n_{i} \times q\right)$ é a matriz de planejamento correspondente ao vetor de efeitos aleatórios $\mathrm{b}_{i}$, de dimensão $(q \times 1)$, e $\epsilon_{i}$ de dimensão $\left(n_{i} \times 1\right)$ é o vetor de erros aleatórios. Assume-se em geral que os efeitos

aleatórios $\mathrm{b}_{i}$ e os componentes residuais $\epsilon_{i}$ são independentes com $\mathrm{b}_{i} \stackrel{i i d}{\sim} N_{q}(\mathbf{0}, \mathbf{D})$ e $\epsilon_{i} \stackrel{i n d}{\sim} N_{n_{i}}\left(0, \Sigma_{i}\right)$, em que $\mathbf{D}=\mathbf{D}(\alpha)$ e $\Sigma_{i}=\Sigma_{i}(\gamma), i=1, \ldots, n$, são matrizes de dispersão, usualmente associadas com a variabilidade entre e intra-unidades amostrais sendo dependentes de um número desconhecido e reduzido de parâmetros $\alpha$ e $\gamma$, respectivamente. Dependendo do contexto, várias suposições podem ser feitas sobre a matriz de covariâncias $\Sigma_{i}$. Com dados agrupados supõe-se, comumente, que $\Sigma_{i}=\sigma_{\epsilon}^{2} \mathbf{I}_{n_{i}} i=1, \ldots, n$, refletindo a suposição de que sub-unidades de um mesmo grupo têm erros permutáveis. Erros permutáveis são comuns em aplicações de dados longitudinais e também em estruturas como AR(1) (Box et al., 1994) e em banda onde $\Sigma_{i}$ depende de um número pequeno de parâmetros.

Seguindo as mesmas idéias de Arellano-Valle et al. (2005), (ver também Genton, 2004), estendemos o modelo linear misto normal (MLMN) definido na Seção 2.1.3, considerando o modelo linear em (5.1) com as seguintes suposições:

$$
\mathbf{b}_{i} \stackrel{i i d}{\sim} S N_{q}(\mathbf{0}, \mathbf{D}, \lambda) \text { e } \epsilon_{i} \stackrel{i n d}{\sim} N_{n_{i}}\left(\mathbf{0}, \Sigma_{i}\right)
$$


em que $\mathbf{b}_{i}$ é independente de $\epsilon_{i}, i=1, \ldots, n$. O parâmetro de assimetria $\lambda$ incorpora a assimetria nos efeitos aleatório $\mathbf{b}_{i}$ e conseqüentemente nas quantidades observadas $\mathrm{Y}_{i}, \quad i=1, \ldots, n$. Se $\boldsymbol{\lambda}=\mathbf{0}$, o modelo assimétrico reduz ao modelo usual de MLMN o qual inferência são extensivamente tratadas na literatura.

Note que de (2.21), e o modelo definido em (5.1)-(5.2) pode ser representado hierarquicamente como

$$
\begin{aligned}
\mathbf{Y}_{i} \mid \mathrm{b}_{i} & \stackrel{\text { ind }}{\sim} N_{n_{i}}\left(\mathbf{X}_{j} \boldsymbol{\beta}+\mathbf{Z}_{i} \mathbf{b}_{i}, \Sigma_{i}\right), \\
\mathbf{b}_{i} \mid T_{i}=t_{i} & \stackrel{\text { ind }}{\sim} N_{q}\left(\lambda t_{i}, \mathbf{D}\right), \\
T_{i} & \stackrel{\text { iid }}{\sim} H N(0,1),
\end{aligned}
$$

$i=1, \ldots, n$, todos independentes. Estratégias para obter uma interpretação correta dos parâmetros do modelo são apresentados em Arellano-Valle et al. (2005). Inferência clássica sobre o vetor de parâmetros $\boldsymbol{\theta}=\left(\boldsymbol{\beta}^{\top}, \boldsymbol{\gamma}^{\top}, \boldsymbol{\alpha}^{\top}, \boldsymbol{\lambda}^{\top}\right)^{\top}$ neste tipo de modelo, é baseado na distribuição marginal para a resposta $\mathbf{Y}_{i}$, dada a seguir

$$
f_{\mathbf{Y}_{i}}\left(\mathbf{y}_{i} \mid \boldsymbol{\theta}\right)=2 \phi_{n_{i}}\left(\mathbf{y}_{i} \mid \mathbf{X}_{i} \boldsymbol{\beta}, \Sigma_{i}+\mathbf{Z}_{i}\left(\mathbf{D}+\boldsymbol{\lambda} \boldsymbol{\lambda}^{\top}\right) \mathbf{Z}_{i}^{\top}\right) \Phi_{1}\left(\bar{\lambda}_{i}\left(\mathbf{y}_{i}-\mathbf{X}_{i} \boldsymbol{\beta}\right)\right)
$$

em que

$$
\bar{\lambda}_{i}=\frac{\lambda^{\top} \mathbf{D}^{-1} \Lambda_{i} \mathbf{Z}_{i}^{\top} \Sigma_{i}}{\sqrt{1+\lambda^{\top} \mathbf{D}^{-1} \lambda+\lambda^{\top} \mathbf{D}^{-1} \Lambda_{i} \mathbf{D}^{-1} \lambda}}, \operatorname{com} \Lambda_{i}=\left(\left(\mathbf{D}+\lambda \boldsymbol{\lambda}^{\top}\right)^{-1}+\mathbf{Z}_{i} \Sigma_{i}^{-1} \mathbf{Z}_{i}\right)^{-1}
$$

$i=1, \ldots, n$. Assim, a função de log-verossimilhança para $\boldsymbol{\theta}$ dada a amostra observada da $\mathbf{y}=\left(\mathbf{y}_{1}^{\top}, \ldots, \mathbf{y}_{n}^{\top}\right)^{\top}$ é dada por $\ell(\boldsymbol{\theta} \mid \mathbf{y})=\sum_{i=1}^{n} \log \left(f_{\mathbf{Y}_{i}}\left(\mathbf{y}_{i} \mid \boldsymbol{\theta}\right)\right)$. A seguir descrevemos um procedimento iterativo para obter as estimativas de máxima verossimilhança (EMV) de $\boldsymbol{\theta}$ baseado no algoritmo EM. 


\subsubsection{Estimação de máxima verossimilhança via algoritmo EM}

Seja $\mathbf{Y}_{c}=\left(\mathbf{y}^{\top}, \mathbf{b}^{\top}, \mathbf{t}^{\top}\right)^{\top} \operatorname{com} \mathbf{y}=\left(\mathbf{y}_{1}^{\top}, \ldots, \mathbf{y}_{n}^{\top}\right)^{\top}, \mathbf{b}=\left(\mathbf{b}_{1}^{\top}, \ldots, \mathbf{b}_{n}^{\top}\right)^{\top}$ e $\mathbf{t}=\left(t_{1}, \ldots, t_{n}\right)^{\top}$. Assim, sob a representação hierarquica (5.3)-(5.5), segue que a função de log-verossimilhança completa associada com $(\mathbf{y}, \mathbf{b}, \mathbf{t})$ é dada por $\ell_{c}\left(\boldsymbol{\theta} \mid \mathbf{Y}_{c}\right)=\sum_{i=1}^{n} \ell_{c i}\left(\boldsymbol{\theta} \mid \mathbf{Y}_{c}\right)$, com

$$
\begin{gathered}
\ell_{c i}\left(\boldsymbol{\theta} \mid \mathbf{Y}_{c}\right)=-\frac{1}{2} \log \left(\left|\Sigma_{i}\right|\right)-\frac{1}{2}\left(\mathbf{y}_{i}-\mathbf{X}_{i} \boldsymbol{\beta}-\mathbf{Z}_{i} \mathbf{b}_{i}\right)^{\top} \boldsymbol{\Sigma}_{i}^{-1}\left(\mathbf{y}_{i}-\mathbf{X}_{i} \boldsymbol{\beta}-\mathbf{Z}_{i} \mathbf{b}_{i}\right) \\
-\frac{1}{2} \log |\mathbf{D}|-\frac{1}{2}\left(\mathbf{b}_{i}-\lambda t_{i}\right)^{\top} \mathbf{D}^{-1}\left(\mathbf{b}_{i}-\lambda t_{i}\right)+C .
\end{gathered}
$$

em que $C$ é uma constante que independe do vetor de parâmetros $\theta$. Sejam $\widehat{\mathrm{b}}_{i}=$ $\mathrm{E}\left[\mathbf{b}_{i} \mid \boldsymbol{\theta}=\widehat{\boldsymbol{\theta}}, \mathrm{y}_{i}\right], \widehat{\Omega}_{i}=\operatorname{Cov}\left[\mathbf{b}_{i} \mid \boldsymbol{\theta}=\widehat{\boldsymbol{\theta}}, \mathrm{y}_{i}\right], \widehat{t_{i}}=\mathrm{E}\left[T_{i} \mid \boldsymbol{\theta}=\widehat{\boldsymbol{\theta}}, \mathrm{y}_{i}\right], \widehat{t}^{2}{ }_{i}=\mathrm{E}\left[T_{i}^{2} \mid \boldsymbol{\theta}=\widehat{\boldsymbol{\theta}}, \mathrm{y}_{i}\right]$ e $\widehat{\mathbf{t b}}_{i}=\mathrm{E}\left[T_{i} \mathbf{b}_{i} \mid \boldsymbol{\theta}=\widehat{\boldsymbol{\theta}}, \mathbf{y}_{i}\right]$ são calculadas de forma similar dao MGSN (ver Apêndice C), obtemos

$$
\begin{aligned}
\widehat{t}_{i} & =\widehat{\mu}_{T i}+W_{\Phi_{1}}\left(\frac{\widehat{\mu}_{T_{i}}}{\widehat{M}_{T_{i}}}\right) \widehat{M}_{T_{i}}, \\
\widehat{t}^{2} & =\widehat{\mu}_{T_{i}}^{2}+\widehat{M}_{T_{i}}^{2}+W_{\Phi_{1}}\left(\frac{\widehat{\mu}_{T_{i}}}{\widehat{M}_{T_{i}}}\right) \widehat{M}_{T_{i}} \widehat{\mu}_{T_{i}}, \\
\widehat{\mathrm{b}}_{i} & =\widehat{\mathrm{r}}_{i}+\widehat{\mathrm{s}}_{i} \widehat{t}_{i}, \\
\widehat{\Omega}_{i} & =\widehat{\mathrm{T}}_{b_{i}}+\widehat{\mathrm{s}}_{i} \widehat{\mathrm{s}}_{i}^{\top}\left(\widehat{t}^{2}{ }_{i}-\left(\widehat{t}_{i}\right)^{2}\right), \\
\widehat{\mathrm{tb}}_{i} & =\widehat{\mathrm{r}}_{i} \widehat{t}_{i}+\widehat{\mathrm{s}}_{i} \widehat{t}^{2}{ }_{i},
\end{aligned}
$$

em que $\widehat{M}_{T_{i}}^{2}=\left[1+\widehat{\lambda}^{\top} \mathbf{Z}_{i}^{\top}\left(\widehat{\Sigma}_{i}+\mathbf{Z}_{i} \widehat{\mathbf{D}} \mathbf{Z}_{i}^{\top}\right)^{-1} \mathbf{Z}_{i} \widehat{\lambda}\right]^{-1}, \widehat{\mu}_{T_{i}}=\widehat{M}_{T_{i}}^{2} \widehat{\lambda}^{\top} \mathbf{Z}_{i}^{\top}\left(\widehat{\Sigma}_{i}+\mathbf{Z}_{i} \widehat{\mathbf{D}} \mathbf{Z}_{i}^{\top}\right)^{-1}\left(\mathbf{y}_{i}-\right.$ $\left.\mathbf{X}_{i} \widehat{\boldsymbol{\beta}}\right), \widehat{\mathbf{T}}_{b_{i}}^{2}=\left[\widehat{\mathbf{D}}^{-1}+\mathbf{Z}_{i}^{\top} \widehat{\Sigma}_{i}^{-1} \mathbf{Z}_{i}\right]^{-1}, \widehat{\mathrm{r}}_{i}=\widehat{\mathbf{T}}_{b_{i}}^{2} \mathbf{Z}_{i}^{\top} \widehat{\Sigma}_{i}^{-1}\left(\mathrm{y}_{i}-\mathbf{X}_{i} \widehat{\boldsymbol{\beta}}\right), \widehat{\mathrm{s}}_{i}=\left(\mathbf{I}_{q}-\widehat{\mathbf{T}}_{b_{i}}^{2} \mathbf{Z}_{i}^{\top} \widehat{\Sigma}_{i}^{-1} \mathbf{Z}_{i}\right) \widehat{\lambda}$, $i=1, \ldots, n$.

A esperança condicional da função de log-verossimilhança completa tem a seguinte expressão:

$$
Q(\boldsymbol{\theta} \mid \widehat{\boldsymbol{\theta}})=E_{\mathbf{b}, \mathbf{T}}\left[\ell_{c}\left(\boldsymbol{\theta} \mid \mathbf{Y}_{c}\right) \mid \mathbf{y}, \widehat{\boldsymbol{\theta}}\right]=\sum_{i=1}^{n} Q_{i}(\boldsymbol{\theta} \mid \widehat{\boldsymbol{\theta}}),
$$


em que

$$
Q_{i}(\boldsymbol{\theta} \mid \widehat{\boldsymbol{\theta}})=Q_{1 i}(\boldsymbol{\beta}, \boldsymbol{\gamma} \mid \widehat{\boldsymbol{\theta}})+Q_{2 i}(\boldsymbol{\alpha}, \boldsymbol{\lambda} \mid \widehat{\boldsymbol{\theta}}),
$$

com

$$
\begin{aligned}
Q_{1 i}(\boldsymbol{\beta}, \boldsymbol{\gamma} \mid \widehat{\boldsymbol{\theta}})= & -\frac{1}{2} \log \left|\boldsymbol{\Sigma}_{i}\right|-\frac{1}{2}\left(\mathbf{Y}_{i}-\mathbf{X}_{i} \boldsymbol{\beta}-\mathbf{Z}_{i} \widehat{\mathrm{b}}_{i}\right)^{\top} \boldsymbol{\Sigma}_{i}^{-1}\left(\mathbf{Y}_{i}-\mathbf{X}_{i} \boldsymbol{\beta}-\mathbf{Z}_{j} \widehat{\mathrm{b}}_{i}\right) \\
& -\frac{1}{2} \operatorname{tr}\left(\boldsymbol{\Sigma}_{i}^{-1} \mathbf{Z}_{i} \widehat{\Omega}_{i} \mathbf{Z}_{i}^{\top}\right)
\end{aligned}
$$

e

$$
Q_{2 i}(\boldsymbol{\alpha}, \boldsymbol{\lambda} \mid \widehat{\boldsymbol{\theta}})=-\frac{1}{2} \log |\mathbf{D}|-\frac{1}{2} \operatorname{tr}\left(\mathbf{D}^{-1}\left(\widehat{\Omega_{i}}+\widehat{\mathbf{b}}_{i} \widehat{\mathbf{b}}_{i}^{\top}-2 \widehat{\mathbf{t b}_{i}} \lambda^{\top}+\widehat{t}_{i}^{2} \lambda \lambda^{\top}\right)\right),(5.10)
$$

em que $\widehat{\mathrm{b}}_{i}, \widehat{\Omega}, \widehat{\mathrm{tb}}_{i}$ como em (5.7) e $\operatorname{tr}(\mathbf{A})$ indica o traço da matriz A.

Os dois passos do algoritmo EM são esquematizados abaixo:

Passo-E: Dado $\boldsymbol{\theta}=\widehat{\boldsymbol{\theta}}$, calculamos $\widehat{t}_{i}, \widehat{t}^{2}{ }_{i}, \widehat{\mathrm{b}}_{i}, \widehat{\Omega}_{i}$ e $\widehat{\mathrm{tb}}_{i}$ para $i=1, \ldots, n$, usando (5.7).

Passo-M: Atualizamos $\widehat{\boldsymbol{\theta}}$ maximizando $Q(\boldsymbol{\theta} \mid \widehat{\boldsymbol{\theta}})$ em relação a $\boldsymbol{\theta}$, que leva à seguinte sequência de passos CM ("constrained maximization") (ver Meng e Rubin, 1993):

Passo-CM 1: Fixando $\gamma=\widehat{\gamma}$, atualizar $\beta$ como

$$
\widehat{\boldsymbol{\beta}}=\left(\sum_{i=1}^{n} \mathrm{X}_{i}^{\top} \widehat{\Sigma}_{i}^{-1} \mathrm{X}_{i}\right)^{-1} \sum_{i=1}^{n} \mathrm{X}_{i} \widehat{\Sigma}_{i}^{-1}\left(\mathrm{y}_{i}-\mathbf{Z}_{i} \widehat{\mathrm{b}}_{i}\right)
$$

Passo-CM 2: Atualizar $\boldsymbol{\lambda}$ como

$$
\widehat{\lambda}=\frac{\sum_{i=1}^{n} \widehat{\mathrm{tb}}_{i}}{\sum_{i=1}^{n}{\widehat{t^{2}}}_{i}}
$$

Passo-CM 3: Fixar $\boldsymbol{\beta}=\widehat{\boldsymbol{\beta}}$ e atualizar $\boldsymbol{\gamma}$ como

$$
\widehat{\boldsymbol{\gamma}}=\operatorname{argmax}_{\boldsymbol{\gamma}}\left\{Q_{1 i}(\widehat{\boldsymbol{\beta}}, \boldsymbol{\gamma} \mid \widehat{\boldsymbol{\theta}})\right\}
$$

Passo-CM 4: Fixar $\lambda=\widehat{\lambda}$ e atualizar $\alpha$ como

$$
\widehat{\boldsymbol{\alpha}}=\operatorname{argmax}_{\boldsymbol{\alpha}}\left\{Q_{2 i}(\boldsymbol{\alpha}, \widehat{\lambda} \mid \widehat{\boldsymbol{\theta}})\right\}
$$


Note que os passos CM-3 e CM-4 requerem apenas a estimação de um tipo de parâmetro, podendo ser efetuados usando o método quase-Newton. Considerando uma situação especial e comum onde $D$ não é estruturado e $\Sigma_{i}=\sigma_{e}^{2} \mathbf{R}_{i}$, com $\mathbf{R}_{i}$ conhecido e $\gamma=\sigma_{e}^{2}$, CM-3 e CM-4, são reduzidos, respectivamente, as seguintes formas fechadas:

$$
\begin{gathered}
\widehat{\sigma_{e}^{2}}=\frac{1}{N} \sum_{i=1}^{n}\left[\left(\mathbf{y}_{i}-\mathbf{X}_{i} \widehat{\boldsymbol{\beta}}-\mathbf{Z}_{i} \widehat{\mathbf{b}}_{i}\right)^{\top} \mathbf{R}_{i}^{-1}\left(\mathbf{y}_{i}-\mathbf{X}_{i} \widehat{\boldsymbol{\beta}}-\mathbf{Z}_{i} \widehat{\mathbf{b}}_{i}\right)+t r\left(\mathbf{R}_{i}^{-1}\left(\mathbf{Z}_{i} \widehat{\Omega}_{i} \mathbf{Z}_{i}^{\top}\right)\right)\right] \\
\widehat{\mathbf{D}}=\frac{1}{n} \sum_{i=1}^{n}\left(\widehat{\Omega}_{i}+\widehat{\mathrm{b}}_{i} \widehat{\mathbf{b}}_{i}^{\top}-2 \widehat{\operatorname{tb}} \widehat{\lambda}_{i}^{\top}+\widehat{t}^{2}{ }_{i} \widehat{\lambda}_{b} \widehat{\lambda}_{b}^{\top}\right) .
\end{gathered}
$$

Quando $\boldsymbol{\lambda}=\mathbf{0}$, as equações do passo-M são reduzidas as expressões de Pinheiro e Bates (2000).

\subsection{Influência local}

Nesta seção desenvolvemos o método de influência local baseado na função Q de Zhu e Lee (2001) definida na Seção 2.5.1, esta metodologia segue da esperança condicional da função de log-verossimilhança de dados completos do algoritmo EM definida em (5.8).

\subsubsection{Cálculo da matriz Hessiana $\ddot{Q}_{\boldsymbol{\theta}}(\widehat{\boldsymbol{\theta}})$}

Seja $\boldsymbol{\theta}=(\boldsymbol{\beta}, \boldsymbol{\gamma}, \boldsymbol{\alpha}, \boldsymbol{\lambda})^{\top}$ o vector de parâmetros do modelo. Para obter as medidas de diagnóstico para influência local de um particular esquema de perturbação, é necessário calcular $\ddot{Q}_{\boldsymbol{\theta}}(\widehat{\boldsymbol{\theta}})=\frac{\partial^{2} Q(\boldsymbol{\theta} \mid \widehat{\boldsymbol{\theta}})}{\partial \boldsymbol{\theta} \partial \boldsymbol{\theta}^{\top}}$. De (5.9)-(5.10), a matriz hessiana é dada 
por $\ddot{Q}_{\boldsymbol{\theta}}(\widehat{\boldsymbol{\theta}})=\sum_{i=1}^{n} \ddot{Q}_{i}(\boldsymbol{\theta}) \mathrm{com}$

$$
\ddot{Q}_{i}(\boldsymbol{\theta})=-\frac{\partial^{2} Q_{i}(\boldsymbol{\theta} \mid \widehat{\boldsymbol{\theta}})}{\partial \boldsymbol{\theta} \partial \boldsymbol{\theta}^{\top}}=\left(\begin{array}{cc}
\ddot{Q}_{1 i}(\boldsymbol{\beta}, \boldsymbol{\gamma}) & 0 \\
0 & \ddot{Q}_{2 i}(\boldsymbol{\alpha}, \boldsymbol{\lambda})
\end{array}\right),
$$

em que

$$
\ddot{Q}_{1 i}(\boldsymbol{\beta}, \boldsymbol{\gamma})=-\frac{\partial^{2} Q_{1 i}(\boldsymbol{\beta}, \boldsymbol{\gamma} \mid \widehat{\boldsymbol{\theta}})}{\partial \tau \partial \boldsymbol{\tau}^{\top}}, \operatorname{com} \boldsymbol{\tau}=\left(\boldsymbol{\beta}^{\top}, \boldsymbol{\gamma}^{\top}\right)^{\top}
$$

e

$$
\ddot{Q}_{2 i}(\boldsymbol{\alpha}, \boldsymbol{\lambda})=-\frac{\partial^{2} Q_{2 i}(\boldsymbol{\alpha}, \boldsymbol{\lambda} \mid \widehat{\boldsymbol{\theta}})}{\partial \pi \partial \boldsymbol{\pi}^{\top}}, \operatorname{com} \pi=\left(\boldsymbol{\alpha}^{\top}, \boldsymbol{\lambda}^{\top}\right)^{\top},
$$

têm elementos dados por (ver Magnus e Neudecker, 1988)

$$
\begin{aligned}
& \frac{\partial^{2} Q_{1 i}(\boldsymbol{\beta}, \gamma \mid \widehat{\boldsymbol{\theta}})}{\partial \boldsymbol{\beta} \partial \boldsymbol{\beta}^{\top}}=-\mathbf{X}_{i}^{\top} \boldsymbol{\Sigma}_{i}^{-1} \mathbf{X}_{i}, \\
& \frac{\partial^{2} Q_{1 i}(\boldsymbol{\beta}, \gamma \mid \widehat{\boldsymbol{\theta}})}{\partial \boldsymbol{\beta} \partial \gamma_{r}}=-\mathbf{X}_{i}^{\top} \boldsymbol{\Sigma}_{i}^{-1} \dot{\boldsymbol{\Sigma}}_{i}(r) \boldsymbol{\Sigma}_{i}^{-1}\left(\mathbf{Y}_{i}-\mathbf{X}_{i} \boldsymbol{\beta}-\mathbf{Z}_{i} \widehat{\mathrm{b}_{i}}\right), \\
& \frac{\partial^{2} Q_{1 i}(\boldsymbol{\beta}, \boldsymbol{\gamma} \mid \widehat{\boldsymbol{\theta}})}{\partial \gamma_{r} \partial \gamma_{s}}=\frac{1}{2} \operatorname{tr}\left\{\boldsymbol{\Sigma}_{i}^{-1}\left(\dot{\boldsymbol{\Sigma}}_{i}(r) \Sigma_{i}^{-1} \dot{\boldsymbol{\Sigma}}_{i}(s)-\ddot{\boldsymbol{\Sigma}}_{i}(r, s)\right)\right\} \\
& -\frac{1}{2} \operatorname{tr}\left\{\mathrm{M}_{\mathbf{i}} \Sigma_{i}^{-1}\left(\dot{\Sigma}_{i}(r) \Sigma_{i}^{-1} \dot{\Sigma}_{i}(s)+\dot{\Sigma}_{i}(s) \Sigma_{i}^{-1} \dot{\Sigma}_{i}(r)-\ddot{\Sigma}_{i}(r, s)\right) \Sigma_{i}^{-1}\right\}, \\
& \frac{\partial^{2} Q_{2 i}(\boldsymbol{\alpha}, \boldsymbol{\lambda} \mid \widehat{\boldsymbol{\theta}})}{\partial \boldsymbol{\lambda} \partial \alpha_{r}}=-\mathbf{D}^{-1} \dot{\mathbf{D}}(r) \mathbf{D}^{-1}\left(\widehat{\mathrm{tb}}_{i}-\widehat{t_{i}^{2}} \boldsymbol{\lambda}\right) \text {, } \\
& \frac{\partial^{2} Q_{2 i}(\boldsymbol{\alpha}, \boldsymbol{\lambda} \mid \widehat{\boldsymbol{\theta}})}{\partial \alpha_{r} \partial \alpha_{s}}=\frac{1}{2} \operatorname{tr}\left\{\mathbf{D}^{-1}\left(\dot{\mathbf{D}}(r) \mathbf{D}^{-1} \dot{\mathbf{D}}(s)-\ddot{\mathbf{D}}(r, s)\right)\right\} \\
& -\frac{1}{2} \operatorname{tr}\left\{\mathbf{N}_{\mathbf{i}} \mathbf{D}^{-1}\left(\dot{\mathbf{D}}(r) \mathbf{D}^{-1} \dot{\mathbf{D}}(s)+\dot{\mathbf{D}}(s) \mathbf{D}^{-1} \dot{\mathbf{D}}(r)-\ddot{\mathbf{D}}(r, s)\right) \mathbf{D}^{-1}\right\}, \\
& \frac{\partial^{2} Q_{2 i}(\boldsymbol{\alpha}, \boldsymbol{\lambda} \mid \widehat{\boldsymbol{\theta}})}{\partial \boldsymbol{\lambda} \partial \boldsymbol{\lambda}^{\top}}=-\widehat{t_{i}^{2}} \mathbf{D}^{-1}, \\
& \text { em que } \mathbf{M}_{\mathbf{i}}=\left(\mathbf{Y}_{i}-\mathbf{X}_{i} \boldsymbol{\beta}-\mathbf{Z}_{i} \widehat{\mathbf{b}}_{i}\right)\left(\mathbf{Y}_{i}-\mathbf{X}_{i} \boldsymbol{\beta}-\mathbf{Z}_{i} \widehat{\mathbf{b}}_{i}\right)^{\top}+\mathbf{Z}_{i} \widehat{\Omega}_{i} \mathbf{Z}_{i}^{\top}, \mathbf{N}_{\mathbf{i}}=\widehat{\Omega}_{i}+\widehat{\mathbf{b}}_{i} \hat{\mathbf{b}}_{i}^{\top}- \\
& 2 \widehat{\mathrm{tb}}_{i} \boldsymbol{\lambda}^{\top}+\widehat{t}^{2}{ }_{i} \boldsymbol{\lambda} \boldsymbol{\lambda}^{\top}, \dot{\Sigma}_{i}(r)=\partial \Sigma_{i} / \partial \gamma_{r}, \ddot{\Sigma}_{i}(r, s)=\partial^{2} \Sigma_{i} / \partial \gamma_{r} \partial \gamma_{s}, r, s=1, \ldots, \operatorname{dim}(\gamma), \\
& i=1, \ldots, n, \dot{\mathbf{D}}(r)=\partial \mathbf{D} / \partial \alpha_{r} \text { e } \ddot{\mathbf{D}}(r, s)=\partial^{2} \mathbf{D} / \partial \alpha_{r} \partial \alpha_{s}, r, s=1, \ldots, \operatorname{dim}(\boldsymbol{\alpha}) \text {. }
\end{aligned}
$$




\subsubsection{Esquemas de perturbação}

Nesta seção consideramos quatro diferentes esquemas de perturbação para o modelo definido em (5.1)-(5.2).

\section{Perturbação da Ponderação de Casos}

Seja $\omega=\left(\omega_{1}, \ldots, \omega_{n}\right)^{\top}$ um vetor $n \times 1$ com $\omega_{o}=(1, \ldots, 1)^{\top}$. O valor esperado da log-verossimilhança de dados completos para o modelo perturbado (função Q perturbada) é expressa por

$$
\begin{aligned}
Q(\boldsymbol{\theta}, \boldsymbol{\omega} \mid \widehat{\boldsymbol{\theta}})= & E\left[\ell_{c}\left(\boldsymbol{\theta}, \boldsymbol{\omega} \mid \mathbf{Y}_{c}\right)\right]=\sum_{i=1}^{n} \omega_{i} E\left[\ell_{i}\left(\boldsymbol{\theta} \mid \mathbf{Y}_{c}\right)\right]=\sum_{i=1}^{n} Q_{1 i}(\boldsymbol{\beta}, \boldsymbol{\gamma}, \boldsymbol{\omega} \mid \widehat{\boldsymbol{\theta}}) \\
& +\sum_{i=1}^{n} Q_{2 i}(\boldsymbol{\alpha}, \boldsymbol{\lambda}, \boldsymbol{\omega} \mid \widehat{\boldsymbol{\theta}}),
\end{aligned}
$$

em que $Q_{1 i}(\boldsymbol{\beta}, \boldsymbol{\gamma}, \boldsymbol{\omega} \mid \widehat{\boldsymbol{\theta}})=w_{i} Q_{1 i}(\boldsymbol{\beta}, \boldsymbol{\gamma} \mid \widehat{\boldsymbol{\theta}})$ e $Q_{2 i}(\boldsymbol{\alpha}, \boldsymbol{\lambda}, \boldsymbol{\omega} \mid \widehat{\boldsymbol{\theta}})=w_{i} Q_{2 i}(\boldsymbol{\alpha}, \boldsymbol{\lambda} \mid \widehat{\boldsymbol{\theta}})$, com $Q_{1 i}(\boldsymbol{\beta}, \boldsymbol{\gamma} \mid \widehat{\boldsymbol{\theta}})$ e $Q_{2 i}(\boldsymbol{\alpha}, \boldsymbol{\lambda} \mid \widehat{\boldsymbol{\theta}})$ como em (5.9) e (5.10), respectivamente.

Neste caso, $\Delta_{\boldsymbol{\omega}_{\circ}}=\left.\frac{\partial^{2} Q(\boldsymbol{\theta}, \boldsymbol{\omega} \mid \widehat{\boldsymbol{\theta}})}{\partial \boldsymbol{\theta} \partial \boldsymbol{\omega}^{\top}}\right|_{\boldsymbol{\omega}=\boldsymbol{\omega}_{\circ}}=\sum_{i=1}^{n} \frac{\partial^{2} Q_{i}\left(\boldsymbol{\theta}, \boldsymbol{\omega}_{o} \mid \widehat{\boldsymbol{\theta}}\right)}{\partial \boldsymbol{\theta} \partial \boldsymbol{\omega}^{\top}}$, tem elementos dados por

$$
\begin{aligned}
& \frac{\partial Q_{1 i}\left(\boldsymbol{\beta}, \boldsymbol{\gamma}, \omega_{o} \mid \widehat{\boldsymbol{\theta}}\right)}{\partial \boldsymbol{\beta} \partial \omega_{i}}=\mathbf{X}_{i}^{\top} \boldsymbol{\Sigma}_{i}^{-1}\left(\mathbf{Y}_{i}-\mathbf{X}_{i} \boldsymbol{\beta}-\mathbf{Z}_{i} \widehat{\mathbf{b}_{i}}\right) \\
& \frac{\partial Q_{1 i}\left(\boldsymbol{\beta}, \boldsymbol{\gamma}, \omega_{o} \mid \widehat{\boldsymbol{\theta}}\right)}{\partial \gamma_{r} \partial \omega_{i}}=-\frac{1}{2} \operatorname{tr}\left\{\boldsymbol{\Sigma}_{i}^{-1} \dot{\boldsymbol{\Sigma}}_{i}(r)-\boldsymbol{\Sigma}_{i}^{-1} \dot{\boldsymbol{\Sigma}}_{i}(r) \boldsymbol{\Sigma}_{i}^{-1} \mathbf{M}_{\mathbf{i}}\right\} \\
& \frac{\partial Q_{2 i}\left(\boldsymbol{\alpha}, \boldsymbol{\lambda}, \omega_{o} \mid \widehat{\boldsymbol{\theta}}\right)}{\partial \alpha_{r} \partial \omega_{i}}=-\frac{1}{2} \operatorname{tr}\left\{\mathbf{D}^{-1} \dot{\mathbf{D}}(r)\right\}+\frac{1}{2} \operatorname{tr}\left\{\mathbf{D}^{-1} \mathbf{N}_{\mathbf{i}} \mathbf{D}^{-1} \dot{\mathbf{D}}(r)\right\}
\end{aligned}
$$

$\mathrm{e}$

$$
\frac{\partial Q_{2 i}\left(\boldsymbol{\alpha}, \lambda, \omega_{o} \mid \widehat{\boldsymbol{\theta}}\right)}{\partial \lambda \partial \omega_{i}}=\mathbf{D}^{-1}\left(\widehat{t \mathbf{b}_{i}}-\widehat{t_{i}^{2}} \lambda\right)
$$

$i=1, \ldots, n, \mathbf{M}_{i}$ e $\mathbf{N}_{i}$ como definido na Seção 5.3 . 
Perturbação da matriz de escala dos efeitos aleatórios

Considerando que a matriz de escala dos efeitos aleatórios é dado por $\mathbf{D}\left(\omega_{i}\right)=$ $\omega_{i}^{-1} \mathbf{D}, i=1, \ldots, n$. Onde assumimos que os efeitos aleatórios são distribuídos heteroscedasticamente como

$$
\mathrm{b}_{i} \sim S N_{q}\left(0, \mathbf{D} / w_{i}, \lambda\right)
$$

A função Q perturbada é da forma

$$
Q(\boldsymbol{\theta}, \boldsymbol{\omega} \mid \widehat{\boldsymbol{\theta}})=\sum_{i=1}^{n} Q_{1 i}(\boldsymbol{\beta}, \boldsymbol{\gamma}, \boldsymbol{\omega} \mid \widehat{\boldsymbol{\theta}})+\sum_{i=1}^{n} Q_{2 i}(\boldsymbol{\alpha}, \boldsymbol{\lambda}, \boldsymbol{\omega} \mid \widehat{\boldsymbol{\theta}})
$$

onde $Q_{1 i}(\boldsymbol{\beta}, \boldsymbol{\gamma}, \boldsymbol{\omega} \mid \widehat{\boldsymbol{\theta}})=Q_{1 i}(\boldsymbol{\beta}, \omega \mid \widehat{\boldsymbol{\theta}}) \mathrm{e}$

$$
Q_{2 i}(\boldsymbol{\alpha}, \boldsymbol{\lambda}, \boldsymbol{\omega} \mid \widehat{\boldsymbol{\theta}})=\frac{q}{2} \log w_{i}-\frac{1}{2} \log |\mathbf{D}|-\frac{w_{i}}{2} \operatorname{tr}\left(\mathbf{D}^{-1}\left(\widehat{\Omega}_{i}+\widehat{\mathbf{b}}_{i} \widehat{\mathbf{b}}_{i}^{\top}-2 \widehat{\operatorname{tb}}_{i} \lambda^{\top}+{\widehat{t^{2}}}_{i} \lambda \lambda^{\top}\right)\right)
$$

Sob este esquema de perturbação, $\omega_{o}=(1, \ldots, 1)^{\top}$ nos leva ao modelo não perturbado. A matriz $\Delta_{\omega_{o}}=\frac{\partial^{2} Q(\boldsymbol{\theta}, \boldsymbol{\omega} \mid \widehat{\boldsymbol{\theta}})}{\partial \boldsymbol{\theta} \partial \boldsymbol{\omega}^{\top}} \mid \boldsymbol{\omega}=\boldsymbol{\omega}_{o}=\frac{\partial^{2} Q\left(\boldsymbol{\theta}, \boldsymbol{\omega}_{o} \mid \widehat{\boldsymbol{\theta}}\right)}{\partial \boldsymbol{\theta} \partial \boldsymbol{\omega}^{\top}}$ tem elementos

$$
\begin{aligned}
\frac{\partial^{2} Q_{1 i}\left(\boldsymbol{\beta}, \boldsymbol{\gamma}, \omega_{o} \mid \widehat{\boldsymbol{\theta}}\right)}{\partial \boldsymbol{\beta} \partial \omega_{i}} & =0, \quad \frac{\partial^{2} Q_{1 i}\left(\boldsymbol{\beta}, \boldsymbol{\gamma}, \omega_{o} \mid \widehat{\boldsymbol{\theta}}\right)}{\partial \gamma \partial \omega_{i}}=0 \\
\frac{\partial^{2} Q_{2 i}\left(\boldsymbol{\alpha}, \boldsymbol{\lambda}, \omega_{o} \mid \widehat{\boldsymbol{\theta}}\right)}{\partial \alpha_{r} \partial \omega_{i}} & =\frac{1}{2} \operatorname{tr}\left\{\mathbf{N}_{i} \mathrm{D}^{-1} \dot{\mathbf{D}}(r) \mathbf{D}^{-1}\right\} \\
\frac{\partial^{2} Q_{2 i}\left(\boldsymbol{\alpha}, \boldsymbol{\lambda}, \boldsymbol{\omega}_{o} / \widehat{\boldsymbol{\theta}}\right)}{\partial \boldsymbol{\lambda} \partial \omega_{i}} & =\mathbf{D}^{-1}\left(\widehat{\mathbf{t b}}_{i}-\widehat{t_{i}^{2}} \boldsymbol{\lambda}\right)
\end{aligned}
$$

$i=1, \ldots, n$.

\section{Perturbação da variável explanatória}

Agora, estamos interessados em perturbar uma variável explicativa contínua. Sob esta condição consideramos a seguinte matriz de variáveis explanatórias perturbadas: $\mathrm{X}_{i \omega}=\left(\mathrm{x}_{i 1}, \ldots, \mathrm{x}_{i k}\left(\omega_{i}\right), \ldots, \mathrm{x}_{i p}\right)$, onde $\mathrm{x}_{i k}\left(\omega_{i}\right)=\mathrm{x}_{i k}+\omega_{i} 1_{n_{i}}, k=1, \ldots, p, \mathrm{x}_{i k}$ é a 
$k$-ésima coluna da matriz $\mathrm{X}_{i}$ e $1_{n_{i}}$ é um vetor de dimensão $n_{i} \times 1$ de uns. Neste caso $\omega_{o}=0$ e $Q_{2 i}(\boldsymbol{\alpha}, \lambda, \omega \mid \widehat{\boldsymbol{\theta}})=Q_{2 i}(\boldsymbol{\alpha}, \boldsymbol{\lambda} \mid \widehat{\boldsymbol{\theta}})$. Como na seção anterior, a matriz

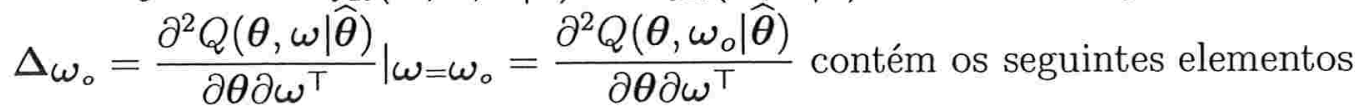

$$
\begin{aligned}
& \frac{\partial^{2} Q_{1 i}\left(\boldsymbol{\beta}, \boldsymbol{\gamma}, \omega_{o} \mid \widehat{\boldsymbol{\theta}}\right)}{\partial \boldsymbol{\beta} \partial \omega_{i}}=\mathbf{f}_{k}\left(\mathbf{Y}_{i}-\mathbf{X}_{i} \boldsymbol{\beta}-\mathbf{Z}_{i} \widehat{\mathrm{b}}_{i}\right)^{\top} \boldsymbol{\Sigma}_{i}^{-1} \mathbf{1}_{n_{i}}-\beta_{k} \mathbf{X}_{i}^{\top} \boldsymbol{\Sigma}_{i}^{-1} \mathbf{1}_{n_{i}}, \\
& \frac{\partial^{2} Q_{1 i}\left(\boldsymbol{\beta}, \boldsymbol{\gamma}, \omega_{o} \mid \widehat{\boldsymbol{\theta}}\right)}{\partial \gamma_{r} \partial \omega_{i}}=-\beta_{k} \mathbf{1}_{n_{i}}^{\top} \boldsymbol{\Sigma}_{i}^{-1} \dot{\boldsymbol{\Sigma}}_{i}(r) \boldsymbol{\Sigma}_{i}^{-1}\left(\mathbf{Y}_{i}-\mathbf{X}_{i} \boldsymbol{\beta}-\mathbf{Z}_{i} \widehat{\mathrm{b}_{i}}\right) \\
& \frac{\partial^{2} Q_{2 i}\left(\boldsymbol{\alpha}, \boldsymbol{\lambda}, \omega_{o} \mid \widehat{\boldsymbol{\theta}}\right)}{\partial \alpha_{r} \partial \omega_{i}}=0, \quad \frac{\partial^{2} Q_{2 i}\left(\boldsymbol{\alpha}, \boldsymbol{\gamma}, \omega_{o} \mid \widehat{\boldsymbol{\theta}}\right)}{\partial \lambda \partial \omega_{i}}=\mathbf{0}
\end{aligned}
$$

$i=1, \ldots, n, \mathbf{f}_{k}$ denota um vetor de dimensão $p \times 1$ de zeros com um na $k$-ésima posição e $\beta_{k}$ denota o $k$-ésimo elemento de $\boldsymbol{\beta}$.

\section{Perturbação da variável resposta}

A perturbação das variáveis resposta $\left(\mathrm{y}_{1}^{\top}, \ldots, \mathrm{y}_{n}^{\top}\right)^{\top}$ é obtida substituindo $\mathrm{y}_{i}$ por $\mathrm{y}_{i} \boldsymbol{\omega}=\mathrm{y}_{i}+\omega_{i} \mathbf{1}_{n_{i}}$, onde $\mathbf{1}_{n_{i}}$ é um vetor de dimensão $n_{i} \times 1$ de uns, $i=1, \ldots, n$. Neste caso, $\omega_{o}=0 \mathrm{e}$

$$
\begin{aligned}
Q_{1 i}(\boldsymbol{\beta}, \boldsymbol{\gamma}, \omega \mid \widehat{\boldsymbol{\theta}})= & -\frac{1}{2} \log \left|\Sigma_{i}\right|-\frac{1}{2}\left(\mathbf{Y}_{i \omega}-\mathbf{X}_{i} \boldsymbol{\beta}-\mathbf{Z}_{i} \widehat{\mathbf{b}}_{i}\right)^{\top} \Sigma_{i}^{-1}\left(\mathbf{Y}_{i \omega}-\mathbf{X}_{i} \boldsymbol{\beta}-\mathbf{Z}_{i} \widehat{\mathbf{b}}_{i}\right) \\
& -\frac{1}{2} \operatorname{tr}\left(\Sigma_{i}^{-1} \mathbf{Z}_{i} \widehat{\Omega}_{i} \mathbf{Z}_{i}^{\top}\right)
\end{aligned}
$$

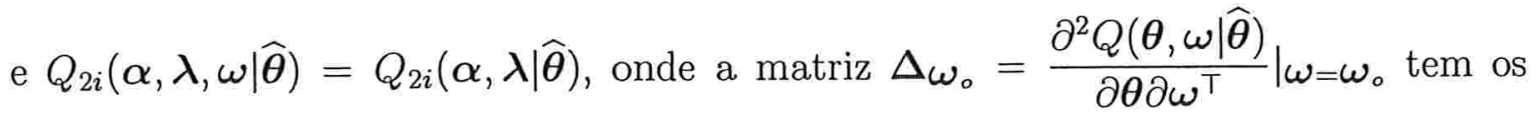
seguintes elementos

$$
\begin{aligned}
& \frac{\partial^{2} Q_{1 i}\left(\boldsymbol{\beta}, \gamma, \omega_{o} \mid \widehat{\boldsymbol{\theta}}\right)}{\partial \boldsymbol{\beta} \partial \omega_{i}}=\mathbf{X}_{i}^{\top} \boldsymbol{\Sigma}_{i}^{-1} \mathbf{1}_{i} \\
& \frac{\partial^{2} Q_{1 i}\left(\boldsymbol{\beta}, \gamma, \omega_{o} \mid \widehat{\boldsymbol{\theta}}\right)}{\partial \gamma_{r} \partial \omega_{i}}=\left(\mathbf{y}_{i}-\mathbf{X}_{i} \boldsymbol{\beta}-\mathbf{Z}_{i} \widehat{\mathrm{b}_{i}}\right) \boldsymbol{\Sigma}_{i}^{-1} \Sigma_{i}(r) \Sigma_{i}^{-1} \mathbf{1}_{i}, \\
& \frac{\partial^{2} Q_{2 i}\left(\boldsymbol{\alpha}, \boldsymbol{\lambda}, \boldsymbol{\omega}_{o} \mid \widehat{\boldsymbol{\theta}}\right)}{\partial \alpha_{r} \partial \omega_{i}}=0, \quad \frac{\partial^{2} Q_{2 i}\left(\boldsymbol{\alpha}, \boldsymbol{\lambda}, \omega_{o} \mid \widehat{\boldsymbol{\theta}}\right)}{\partial \lambda \partial \omega_{i}}=0 .
\end{aligned}
$$




\subsection{Estudo de simulação}

Para investigar o desempenho empírico das medidas propostas, consideramos o seguinte modelo linear misto normal-assimétrico

$$
y_{i j}=\beta_{0}+\beta_{1} x_{i j}+b_{j} z_{i j}+\epsilon_{i j}
$$

onde $j=1, \ldots, 5, i=1, \ldots, 200$. Tomamos, $t_{i j}=j-3, w_{i}=1$ se $i \leq 100 \mathrm{e}$ $w_{i}=0$ if $i>100, \beta_{1}=2, \beta_{2}=1, \epsilon_{i j} \sim N\left(0,0.5^{2}\right)$ e $\beta_{o}+b_{i} \sim S N_{1}\left(0,0.2^{2}, 0.3\right)$, o que resulta dados altamente assimétricos. Agora, consideramos os seguintes pontos atípicos $\left\{y_{i j}: j=1, \ldots, 5, i=5,20,50,150\right\}$ onde os $b_{i}$ são substituídos (e fixados) por $b_{i}=2$, para $i=5,20,50,150$. Com todas essas informações, geramos um conjunto de dados incluindo dados atípicos considerando um MLMSN definida em (5.11). Desta forma, seguimos os métodos propostos na Seção 5.5. Na Figura 5.1 apresentamos os gráficos de índices $\left|C_{i}\right|$ para perturbação de ponderação de casos e das variáveis respostas, $|d \max |$ para perturbação da variável de escala dos efeitos aleatórios, $\left|C_{i}\right|$ para a perturbação da variável resposta e para o análogo da veossimilhança afastada $\left(L D_{i}^{c}\right)$. Note que, em todos estes casos os pontos $5,20,50$ e 150 foram escolhidos corretamente indicando que a metodologia funciona muito bem, quando um ponto suspeito está presente no modelo. 
Figura 5.1: Conjunto de dados simulados. Gráfico de índice (a) $C_{i}$ para perturbação de ponderação de casos, (b) $\mid$ dmax $\mid$ para perturbação da matriz de escala da efeitos aleatórios, (c) $C_{i}$ para perturbação da variável resposta e (d) afastamento da verossimilhança $L D_{i}^{c}$.

(a)

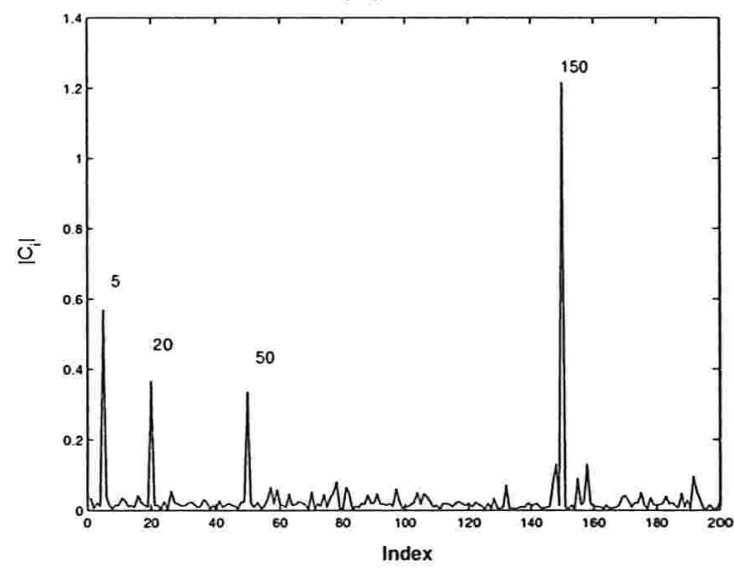

(c)

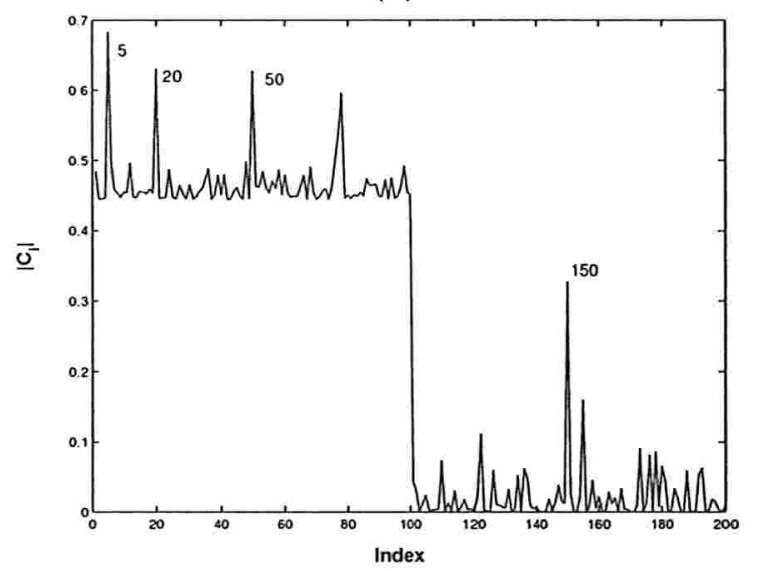

(b)

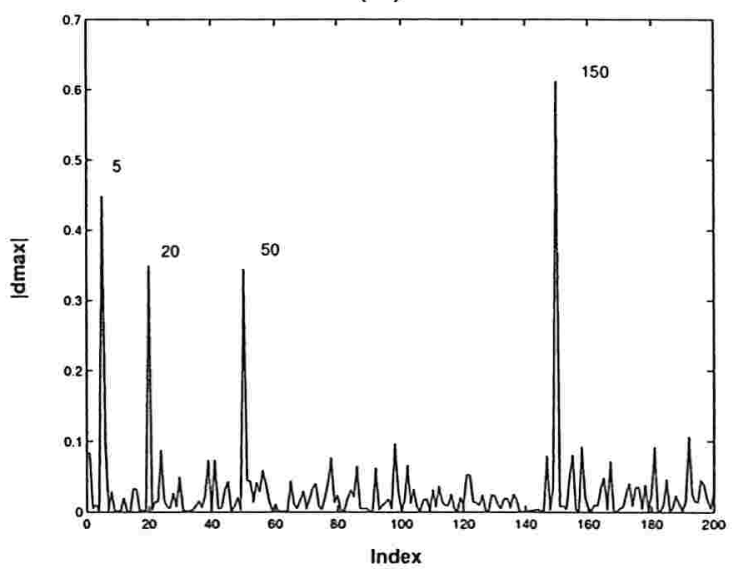

(d)

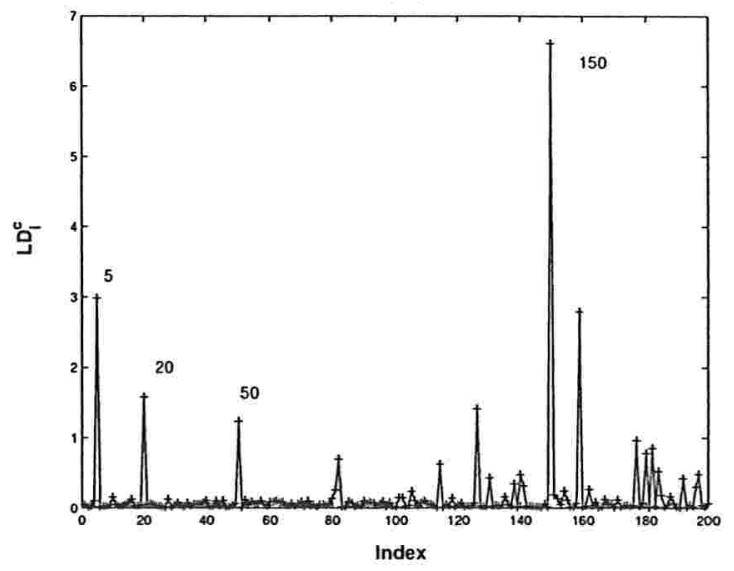

\subsection{Aplicação}

Nesta seção ilustramos a utilidade dos métodos propostos aplicando a dados longitudinais coletados como parte do conhecido estudo de colesterol de Framingham (Zhang e Davidian, 2001). O conjunto de dados inclui os níveis de colesterol em diferentes 
instantes do tempo, idade no início do estudo e sexo para $n=200$ indivíduos selecionados aleatoriamente, registrados em Zhang e Davidian (2001). Adotamos o mesmo modelo linear utilizado pelos autores, dado por

$$
y_{i j}=\beta_{o}+\beta_{1} \operatorname{sexo}_{j}+\beta_{2} i d a d e_{j}+\beta_{3} t_{i j}+b_{o j}+b_{1 j} t_{i j}+\epsilon_{i j}
$$

onde $y_{i j}$ representa o nível de colesterol $(\mathrm{mg} / \mathrm{dL})$ dividido por 100 no $j$-ésimo tempo para o indivíduo $i$ e $t_{i j}$ é (tempo - 5)/10, com tempo medido em anos; $i d a d e_{i}$ é a idade no início do estudo (em anos); sexo $o_{i}$ é o indicador do sexo $(0=$ feminino, $1=$ masculino). Assim, $\mathbf{x}_{i j}=\left(1, \text { idade }_{i}, \operatorname{sexo}_{i}, t_{i j}\right)^{T}, \mathbf{b}_{i}=\left(b_{o i}, b_{1 i}\right)^{T}$ e $\mathbf{Z}_{i}=\left(1, t_{i j}\right)^{T}$. Os gráficos na Figura 5.2 (a) e (b) mostram o histograma dos níveis de colesterol e gráfico de contorno, respectivamente, indicando claramente que os dados seguem um comportamento assimétrico e que parece adequado ajustar um modelo normalassimétrico para este conjunto de dados. Zhang e Davidian (2001) analisaram este conjunto de dados e ressultaram que o comportamento assimétrico é parcialmente explicado pelas covariáveis e efeitos aleatórios, o qual não podem ser normalmente distribuído. O modelo aqui considerado é um modelo com distribuição normal para os erros e distribuição normal-assimétrica multivariada para os efeitos aleatórios $\mathbf{b}_{i}$, com $\boldsymbol{\lambda}=\left(\lambda_{b 1}, \lambda_{b 2}\right)^{\top}$. Neste caso consideramos $\psi_{i}=\sigma_{e}^{2} \mathbf{I}_{n_{i}}, i=1, \ldots, 200$, como a matriz de covariância dos erros (modelo de independência condicional). A Tabela 5.1 apresenta os resultados obtidos usando o algoritmo tipo-EM do modelo descrito acima. Os erros padrão assintóticos (EP) obtidos numericamente a partir da matriz hessiana. Os critérios de informação, Akaike (AIC), Schwarz (BIC) e Hannan-Quinn (HQ) indicam que o MLMSN apresenta melhor ajuste comparado ao MLMN, justificando a suposição de que os dados têm um comportamento assimétrico. Note-se que as estimativas dos coeficientes $\beta_{1}, \beta_{2}$ e $\beta_{3}$, também diferem.

Para este conjunto de dados o estudo de influência local baseia-se em perturbação 
de casos com interesse em $\boldsymbol{\theta}$. Os resultados são mostrados na Figura 5.3. Os indivíduos 90 e 146 parecem ser bastantes influentes revelado descrito na Figura 5.3 (a). No entanto, os indivíduos 39, 90, 146 e 160 parecem ser influentes usando influência local total $C_{i}$ como mostra na Figura 5.3 (b). Além disso, os gráficos (a) e (b) da Figura 5.4 mostram que os indivíduos 39 e 160 parecem ser os mais influentes quando perturbamos a matriz de escala dos efeitos aleatórios e nos gráficos (c) e (d) da Figura 5.4, respectivamente, indicam que os índivíduos 39 e 90 são os mais influentes quando a variável explanatória e resposta são perturbadas. Em geral, nota-se que o indivíduo 39 parece ser o mais influente, pelo fato de o nível de colesterol encontrarse abaixo da média em relação aos outros indivíduos e considerando-se que a idade desse indivíduo é de 59 anos (nesta idade espera-se que o nível de colesterol supere à média). Complementando esta afirmação, podemos notar que este indivíduo parce ser globalmente influente usando afastamento da verossimilhança $\left(L D_{i}^{c}\right)$ ou distância de Cook $\left(D_{i}^{c}\right)$, mostrados na Figura 5.5 (a) e (b), respectivamente. 
Tabela 5.1: Resultados do ajuste do modelo misto normal-assimétrico e normal (MLMSN e MLMN) para o conjunto de dados de colesterol de Framingham. $d_{11}$, $d_{12}$ e $d_{22}$ são os distintos elementos da matriz de covariâncias $\mathbf{D}^{1 / 2}$. EP são erros padrão estimados assintóticamente baseados na matriz hessiana.

\begin{tabular}{ccccc} 
& \multicolumn{3}{c}{ MLMSN } & \multicolumn{2}{c}{ MLMN } \\
Parâmetros & Estimativas & EP & Estimativas & EP \\
\hline$\beta_{o}$ & 1.3555 & 0.1397 & 1.5967 & 0.1543 \\
$\beta_{1}$ & -0.0484 & 0.0496 & -0.0631 & 0.0568 \\
$\beta_{2}$ & 0.0150 & 0.0035 & 0.0184 & 0.0037 \\
$\beta_{3}$ & 0.3541 & 0.0555 & 0.2817 & 0.0242 \\
$\sigma_{e}^{2}$ & 0.0429 & 0.0024 & 0.0434 & 0.0024 \\
$d_{11}$ & 0.1875 & 0.0547 & 0.3716 & 0.0201 \\
$d_{12}$ & 0.1363 & 0.0380 & 0.0563 & 0.0179 \\
$d_{22}$ & 0.1434 & 0.0518 & 0.1868 & 0.0329 \\
$\lambda_{b 1}$ & 0.4776 & 0.0673 & - & - \\
$\lambda_{b 2}$ & -0.0918 & 0.0633 & - & - \\
\hline -log-likelihood & -668.7083 & -659.7553 \\
AIC & -658.6596 & -651.7692 \\
BIC & -633.9168 & -631.9332 \\
HQ & -649.2636 & -644.2524 \\
\hline \hline
\end{tabular}


Figura 5.2: Conjunto de dados de Colesterol de Framingham. (a) Histograma dos níveis de colesterol sobreposta pela densidade estimada kernel e (b) gráfico de contornos da densidade estimada de $\mathbf{b}_{i}$.

(a)

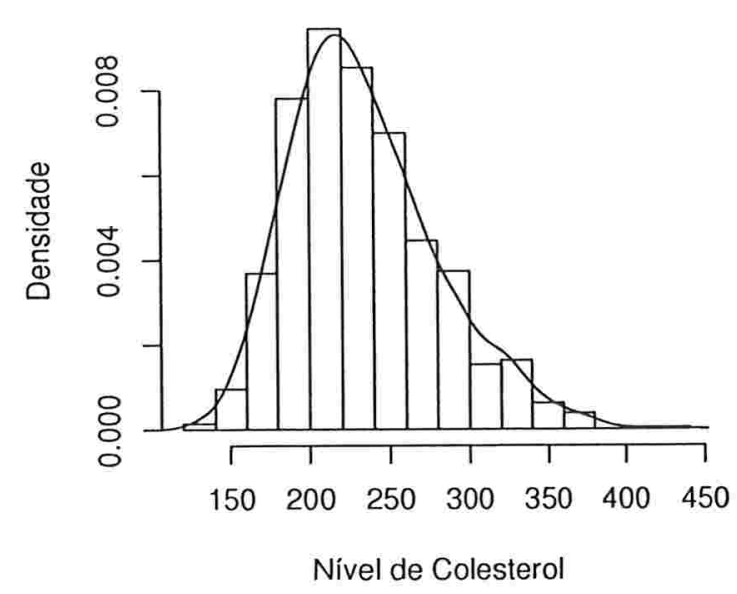

(b)

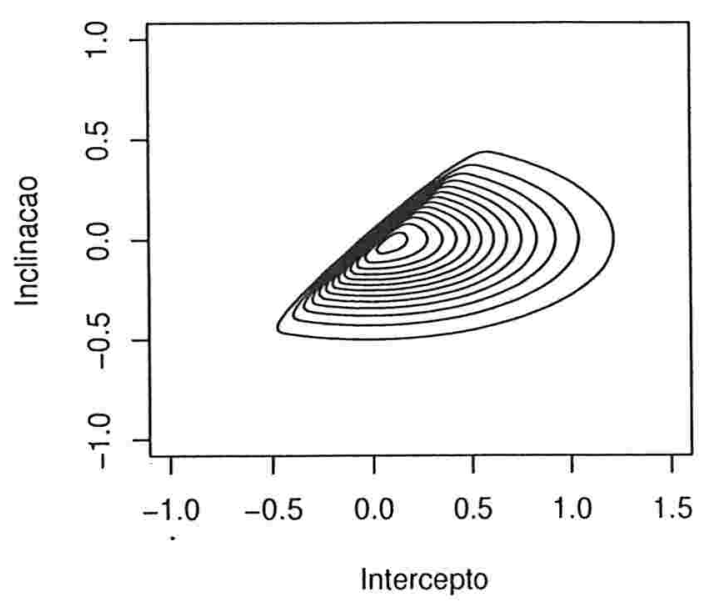

Figura 5.3: Conjunto de dados de Colesterol de Framingham, interesse em $\boldsymbol{\theta}$. Gráfico de índice: (a) $|d \max |$ e (b) $\left|C_{i}\right|$ para perturbação da ponderação de casos

(a)

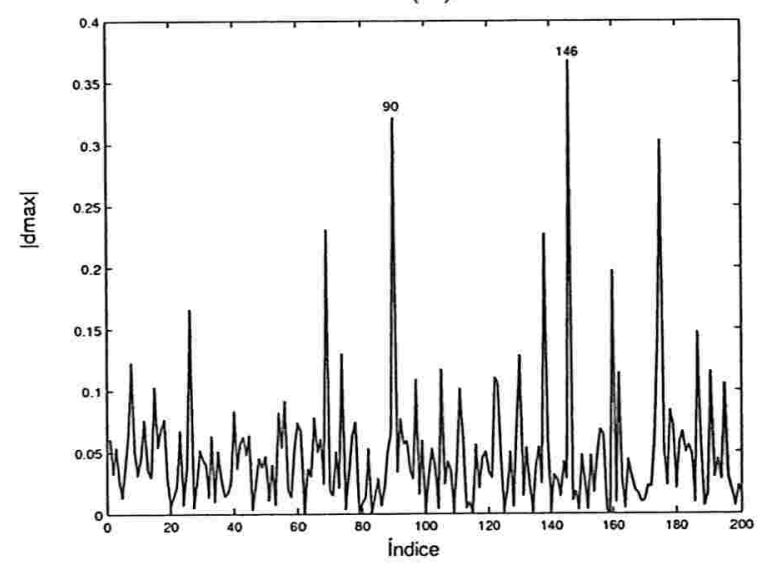

(b)

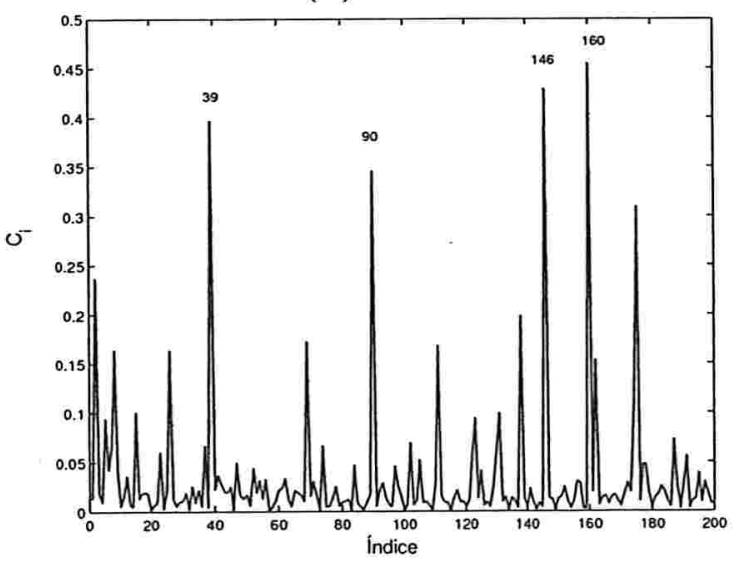


Figura 5.4: Conjunto de dados de Colesterol de Framingham, interesse em $\boldsymbol{\theta}$. Gráfico de índice: (a) $|d \max |$, (b) $\left|C_{i}\right|$ para perturbação da matriz de escala dos efeitos aleatórios, (c) $|d \max |$ para perturbação das variáveis explanatórias e (d) $\left|C_{i}\right|$ para perturbação das variáveis respostas.

(a)

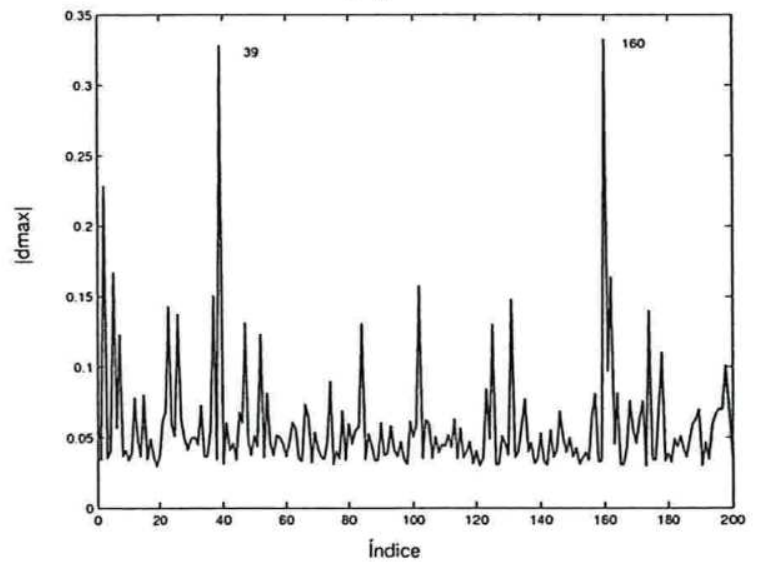

(c)

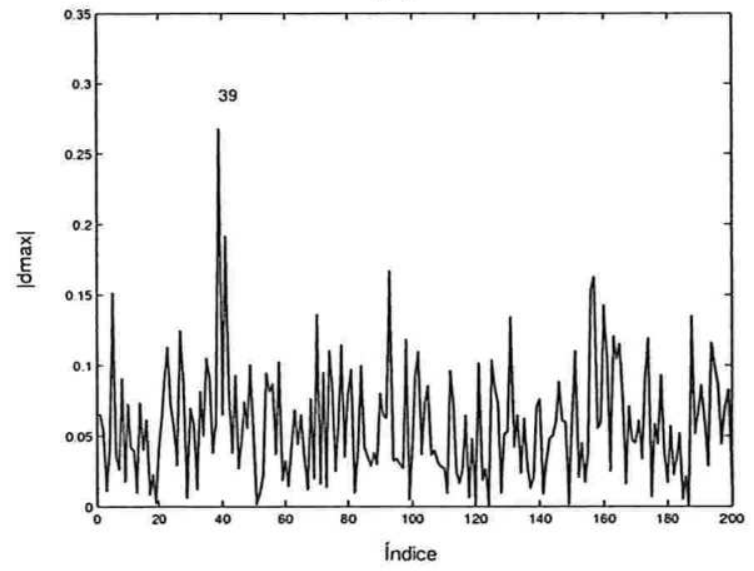

(b)

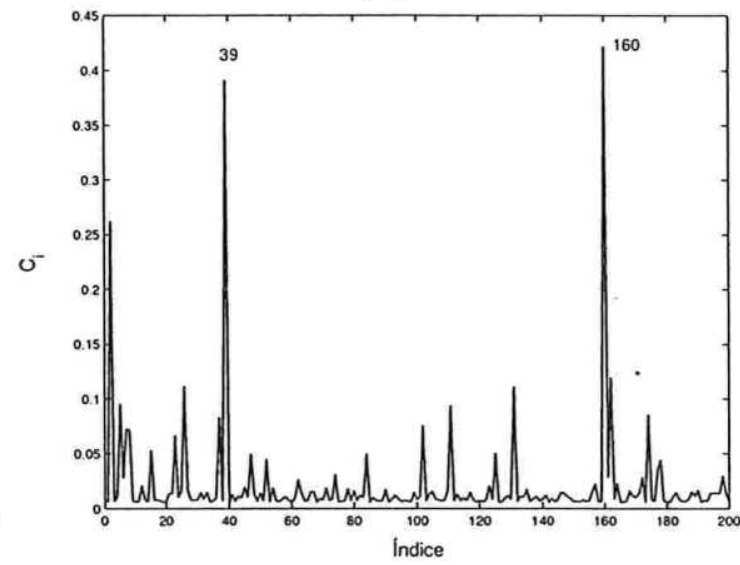

(d)

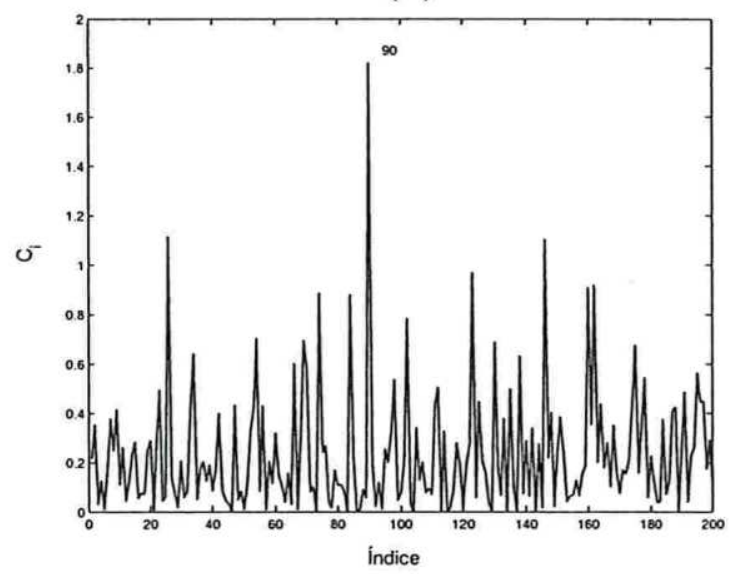


Figura 5.5: Conjunto de dados de Colesterol de Framingham. Gráfico de medidas da (a) Afastamento da verossimilhança $L D_{i}^{c}$ e (b) distância de Cook $D_{i}^{c}$.

(a)

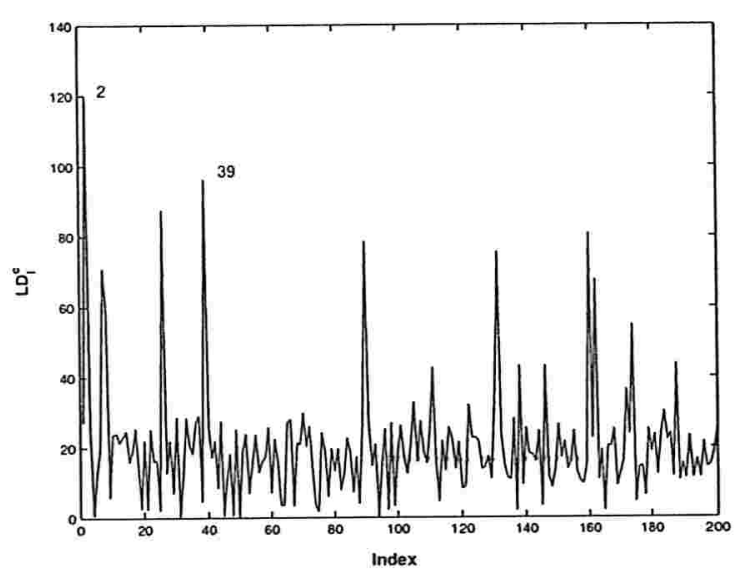

(b)

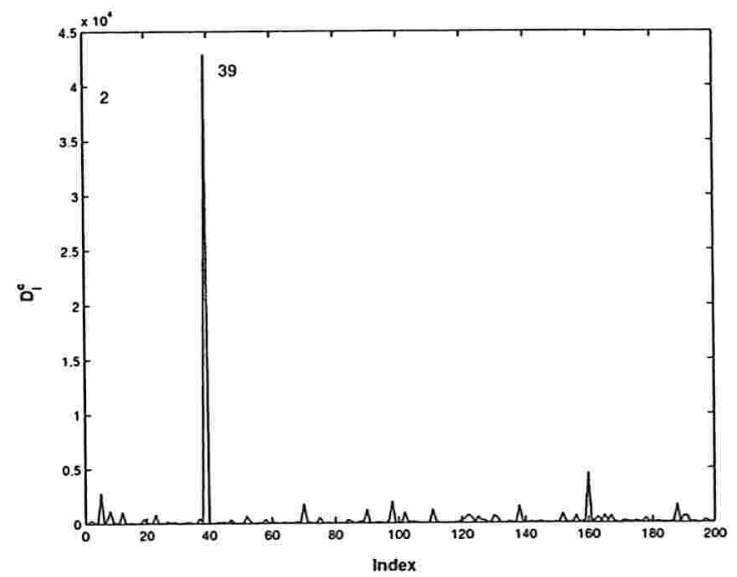




\section{Capítulo 6}

\section{Modelo de tempo de falha}

\section{acelerada com erro de medida}

\subsection{Introdução}

Existem diversos estudos na literatura que tratam de problema de covariáveis com erro de medida, sendo quase todos com base na verossimilhança parcial usada no contexto de Cox (1972). Um dos primeiros estudos nesta área é apresentado por Prentice (1982). Hughes (1993) estuda o viés assintótico do estimador obtido da função de verossimilhança parcial. Nakamura (1992) aplica o método do escore corrigido ao modelo de Cox quando os erros de medida são considerados aditivos e normalmente distribuídos. $\mathrm{Hu}$ et al. (1998) apresentam vários métodos diferentes para estimar os parâmetros do modelo de Cox com diferentes suposições em relação à distribuição das covariáveis verdadeiras. Em relação aos modelos de tempo de falha acelerado com erro de medida, temos alguns resultados para o modelo de regressão 
Weibull. Gimenez et al. (1999) apresentam um estudo de simulação para comparar propriedades de diferentes estimadores do coeficiente angular do modelo com diferentes níveis de censura. Gimenez (1997) mostra que o modelo de regressão Weibull possui uma representação exata da função escore corrigida. Estudos referentes a modelos de tempo de falha acelerado com efeito aleatório (ou fragilidade) para o modelo de Cox são encontrados por exemplo no trabalho de Vaupel et al. (1979). Estes autores construíram um modelo baseado na função de risco em uma análise univariada utilizando o método de tábua de vida (Lawless, 1982). Eles modelaram a variável de fragilidade de maneira multiplicativa uzando a distribuição gama. Clayton e Cuzick (1985) estenderam o modelo de Clayton para incluir covariáveis fixas. Para o modelo Weibull, Valença (2003) apresenta um método de estimação que pode ser facilmente utilizado através do procedimento MLMIXED do sistema SAS (Littell et al., 1996). Sahu e Dey (2000) comparam modelos de fragilidade para dados de sobrevivência de distribuição Weibull e exponencial bivariada. Considerando o modelo de regressão normal multivariado citamos o artigo de Klein et al. (1999), entre outros.

Neste capítulo, investigamos a ocorrência de erros de medida em modelos de sobrevivência para dados não correlacionados. Adotamos o modelo de tempo de falha acelerado com distribuição log-normal para o tempo e consideramos a estimação dos coeficientes do modelo de regressão, quando existem variáveis medidas com erros. Para o modelo de sobrevivência para dados não correlacionados, o interesse é obter a distribuição marginal das quantidades observadas e utilizar alguns métodos de estimação, como máxima verossimilhança por meio dos métodos numéricos de maximização e pelo algoritmo EM, pelo método de simulação e extrapolação (SIMEX) e por regressão-calibração, bem como comparar a eficiência de cada método. É apresentado também a estimação da função de sobrevivência. Para dados correlacionados, os 
estimadores de máxima verossimilhança são obtidos por meio de métodos numéricos de máximização e adicionalmente um estudo bayesiano é apresentado com o intuito de comparar os resultados obtidos no caso clássico.

\subsection{Modelo sem efeito aleatório}

Consideremos uma amostra aleatória de tamanho $n$ e sejam $t_{1}, \ldots, t_{n}$ variáveis aleatórias não negativas e independentes representando tempos de vida $\left(T_{i}\right)$ ou instantes de censura $\left(C_{i}\right)$ para o $i$-ésimo indivíduo. Seja $x_{i}$ (escalar) uma covariável que não pode ser observada diretamente, mas em seu lugar temos $w_{i}$. O modelo log-linear para $Z_{i}=\log T_{i}$, com erro de medida é dado por

$$
\log T_{i}=\alpha+\beta x_{i}+\epsilon_{i}
$$

sendo $\epsilon_{i}$ erros aleatórios independentes e identicamente distribuídos. Os parâmetros do modelo são $\alpha$ e $\beta$. Em um modelo com erro de medida aditivo temos

$$
w_{i}=x_{i}+\xi_{i}
$$

$\operatorname{com} \xi_{i}$ representando erros de medida aleatórios independente e identicamente distribuidos. Assumimos que estes erros possuem distribuição normal com média zero e variância $\sigma_{\xi}^{2}$.

Diferentes distribuições estabelecidas para os erros $\epsilon_{i}$ definem diferentes modelos. Embora nosso interesse seja aplicar os resultados para o modelo log-normal, isto é, assumindo $\epsilon_{i}$ com distribuição normal, os métodos a serem considerados a seguir, são apresentados para toda a classe de modelos definidos em (6.1) e (6.2). Assumimos em nosso estudo um modelo estrutural, ou seja, as covariáveis $x_{i}, i=1, \ldots, n$ são considerados independentes e identicamente distribuídas com uma distribuição normal de 
média $\mu_{x}$ e variância $\sigma_{x}^{2}$, supomos também que $x_{i}, \epsilon_{i}$ e $\xi_{i}$ sejam mutuamente independentes. Consideramos o modelo definido acima e sendo $\theta=\left(\alpha, \beta, \mu_{x}, \sigma_{\epsilon}^{2}, \sigma_{x}^{2}, \sigma_{\xi}^{2}\right)^{\top}$ o vetor de parâmetros desconhecidos a ser estimado temos problemas de identificabilidade e, portanto, não existirem estimadores consistentes para $\alpha$ e $\beta$. Para contornarmos este problema de identificabilidade, assumimos um procedimento mais utilizado envolvendo a suposição de que $\sigma_{\xi}^{2}$ é conhecida. Esta suposição reduz o número de parâmetros para $\theta=\left(\alpha, \beta, \mu_{x}, \sigma_{\epsilon}^{2}, \sigma_{x}^{2}\right)^{\top}$. Admitimos que os tempos de sobrevivência estão sujeitos a censura à direita, assumiremos que a censura é aleatória e não informativa, ou seja, a sua distribuição não depende de parâmetros de interesse. Consideramos também $Y_{i}=\min \left(\log T_{i}, \log C_{i}\right)$, o indicador de falha $\delta_{i}$ definido como $\delta_{i}=I\left(T_{i} \leq C_{i}\right)$, sendo $I($.$) a função indicadora. Assim, os dados são representados$ pelos pares de variáveis aleatórias $\left(Y_{i}, \delta_{i}\right)$ e pela covariável observada $w_{i}$. O modelo definido acima.pode ser expresso como

$$
\begin{aligned}
Y_{i} \mid x_{i} \sim N\left(\alpha+\beta x_{i} ; \sigma_{\epsilon}^{2}\right), \\
w_{i} \mid x_{i} \sim N\left(x_{i} ; \sigma_{\xi}^{2}\right), \\
x_{i} \sim N\left(\mu_{x} ; \sigma_{x}^{2}\right) .
\end{aligned}
$$

Para se obter a densidade conjunta de $\left(Y_{i}, w_{i}\right)$ que é a amostra observada, temos que eliminar $x_{i}, i=1, \ldots, n$. Efetuamos isso integrando em $x_{i}$, como mostramos a seguir. A função de densidade conjunta de $\left(Y_{i}, w_{i}\right)$ para observações não censuradas pode ser expressa como

$$
\begin{aligned}
f\left(y_{i}, w_{i}\right) & =\int_{-\infty}^{+\infty} f\left(y_{i}, w_{i}, x_{i}\right) d x_{i} \\
& =\int_{-\infty}^{+\infty} f\left(y_{i} \mid w_{i}, x_{i}\right) f\left(w_{i}, x_{i}\right) d x_{i} \\
& =\int_{-\infty}^{+\infty} f\left(y_{i} \mid x_{i}\right) f\left(w_{i} / x_{i}\right) f\left(x_{i}\right) d x_{i}
\end{aligned}
$$


em que a igualdade na última passagem deve-se à do modelo não diferenciável (Bolfarine e Arellano-Valle, 1998). Assim, após algumas manipulações algébricas, obtemos

$$
\left(\begin{array}{c}
y_{i} \\
w_{i}
\end{array}\right) \stackrel{i i d}{\sim} N_{2}\left[\left(\begin{array}{c}
\alpha+\beta \mu_{x} \\
\mu_{x}
\end{array}\right) ;\left(\begin{array}{cc}
\beta^{2} \sigma_{x}^{2}+\sigma_{\epsilon}^{2} & \beta \sigma_{x}^{2} \\
\beta \sigma_{x}^{2} & \sigma_{x}^{2}+\sigma_{\xi}^{2}
\end{array}\right)\right] .
$$

Para as observações censuradas, temos

$$
\begin{aligned}
P\left(Y_{i} \geq y_{i}, w_{i}\right) & =\int_{y_{i}}^{+\infty} f\left(z_{i}, w_{i}\right) d z_{i} \\
& =\int_{-\infty}^{+\infty}\left[\int_{y_{i}}^{+\infty} f\left(z_{i}, w_{i}, x_{i}\right) d z_{i}\right] d x_{i} \\
& =\int_{-\infty}^{+\infty} \int_{y_{i}}^{+\infty} f\left(z_{i} \mid w_{i}, x_{i}\right) f\left(w_{i} \mid x_{i}\right) f\left(x_{i}\right) d z_{i} d x_{i} \\
& =\int_{-\infty}^{+\infty} \int_{y_{i}}^{+\infty} f\left(z_{i} \mid x_{i}\right) f\left(w_{i} \mid x_{i}\right) f\left(x_{i}\right) d z_{i} d x_{i} \\
& =\int_{y_{i}}^{+\infty} \int_{-\infty}^{+\infty} f\left(z_{i} \mid x_{i}\right) f\left(w_{i} \mid x_{i}\right) f\left(x_{i}\right) d x_{i} d z_{i} \\
& =\int_{y_{i}}^{+\infty} \int_{-\infty}^{+\infty} f\left(z_{i}, w_{i}, x_{i}\right) d x_{i} d z_{i} \\
& =\int_{y_{i}}^{+\infty} f\left(z_{i} \mid w_{i}\right) f\left(w_{i}\right) d z_{i} \\
& =f\left(w_{i}\right) S\left(y_{i} \mid w_{i}\right)
\end{aligned}
$$

em que $S\left(y_{i} \mid w_{i}\right)=1-\Phi\left(y_{i} ; \mu_{c i}, \sigma_{c}^{2}\right)$, com $\mu_{c i}=\alpha+\beta \mu_{x}+\frac{\beta \sigma_{x}^{2}}{\sigma_{x}^{2}+\sigma_{\xi}^{2}}\left(w_{i}-\mu_{x}\right)$ e $\sigma_{c}^{2}=$ $\beta^{2} \sigma_{x}^{2}\left(\frac{\sigma_{\xi}^{2}}{\sigma_{x}^{2}+\sigma_{\xi}^{2}}\right)+\sigma_{\epsilon}^{2}$ denotam a média e a variância condicionais de $Y_{i}$ dado $w_{i}, i=$ $1, \ldots, n, \Phi($.$) denota a função de distribuição acumulada da distribuição N\left(\mu_{c i}, \sigma_{c}^{2}\right)$. Portanto, a função de verossimilhança pode ser obtida a partir das expressões (6.6) e (6.7) para as observações não censuradas e censuradas, respectivamente. 


\subsubsection{Estimação dos parâmetros}

Nesta seção discutimos a estimação dos parâmetros através de quatro métodos diferentes de estimação. O primeiro é o método de máxima verossimilhança em que os estimadores são obtidos a partir da maximização direta da função de verossimilhança e pelo algoritmo EM. O segundo método considerado é o método de regressãocalibração em que os estimadores são obtidos fazendo a troca da covariável não observada $x$ por um estimador da esperança condicional de $x$ dado $(w, y)$ e o uso de um procedimento padrão para ajustar um modelo com dados censurados. O terceiro é o método de simulação e extrapolação (SIMEX) em que os estimadores são obtidos experimentalmente via simulação seguida de extrapolação. Finalmente, o quarto método considerado é o método "ingênuo"em que os estimadores são obtidos fazendo a substituição da variável não observada $x$ pela variável observada $w$, ignorando os erros de medição. Contudo, este método nos proprociona estimadores viesados e inconsistentes, sendo apresentado apenas com o intuito de comparação com os demais procedimentos. 


\section{Método de máxima verossimilhança}

A função de verossimilhança correspondente a $\theta$ dada a amostra observada $\mathrm{y}=$ $\left(y_{1}, \ldots, y_{n}\right), \mathrm{w}=\left(w_{1}, \ldots, w_{n}\right)$ e $\delta=\left(\delta_{1}, \ldots, \delta_{n}\right)$ segundo (6.6) e (6.7) é dada por

$$
\begin{aligned}
L(\boldsymbol{\theta} ; \mathbf{y}, \mathbf{w})= & \prod_{i=1}^{n} f\left(y_{i} / w_{i}\right)^{\delta_{i}} S\left(y_{i} \mid / w_{i}\right)^{1-\delta_{i}} f\left(w_{i}\right) \\
= & \prod_{i \in F} f\left(y_{i} \mid w_{i}\right) \prod_{j \in C} S\left(y_{i} \mid w_{i}\right) \prod_{i=1}^{n} f\left(w_{i}\right) \\
= & \prod_{i \in F} \phi\left(y_{i}: \mu_{c i}, \sigma_{c}^{2}\right) \prod_{i \in C}\left[1-\Phi\left(y_{i}: \mu_{c i}, \sigma_{c}^{2}\right)\right] \\
& \times \prod_{i=1}^{n} \phi\left(w_{i}: \mu_{x}, \sigma_{x}^{2}+\sigma_{\xi}^{2}\right),
\end{aligned}
$$

onde $F$ e $C$ denotam os conjuntos de indivíduos onde $y_{i}$ é o log do tempo de vida observada e o log do tempo de censura observada, respectivamente, $\mu_{c i}$ e $\sigma_{c}^{2}$ foram definidas na Seção 6.2. Tomando o logaritmo da verossimilhança em (6.8) tem-se que

$$
\begin{aligned}
l(\boldsymbol{\theta})= & \sum_{i \in F}\left\{-\frac{1}{2} \log \sigma_{c}^{2}-\frac{1}{2 \sigma_{\epsilon}^{2}}\left(y_{i}-\mu_{c i}\right)^{2}\right\} \\
& +\sum_{i \in C} \log \left\{1-\Phi\left(\frac{y_{i}-\mu_{c i}}{\sigma_{\epsilon}}\right)\right\} \\
& -\frac{1}{2} \sum_{i=1}^{n}\left\{\log \left(\sigma_{x}^{2}+\sigma_{\xi}^{2}\right)+\frac{1}{2\left(\sigma_{x}^{2}+\sigma_{\epsilon}^{2}\right)}\left(w_{i}-\mu_{x}\right)^{2}\right\}+C .
\end{aligned}
$$

onde $C$ é uma constante que independe do vetor de parâmetros $\boldsymbol{\theta}$. Os estimadores de máxima verossimilhança do vetor de parâmetros $\theta$ não apresentam uma solução em forma fechada. Assim, será nescessário obter os estimadores através de maximização numérica da função log-verossimilhança, utilizando um processo iterativo. O método utilizado é chamado L-BFGS-B desenvolvido por Byrd et al. (1995) que nos permite utilizar restrições antes de estimar os parâmetros. Este método é baseado no método de projeção do gradiente e usa um método quase Newton conhecido como 
BFGS desenvolvido pelo trabalho individual de Broyden (1970), Fletcher (1970), Goldfarb (1970) e Shanno (1970) que nos permite aproximar a matrix hessiana da função de interesse. L-BFGS-B está implementado na linguagem de programação R (Chambers, 2005) através da função de minimização optim. A matriz de covariâncias assintóticas dos estimadores de máxima verossimilhança pode ser estimada usando a matriz hessiana que pode ser calculada também numericamente. Enfatizamos, que o cálculo da matriz hessiana numericamente é uma vantagem do método de máxima verossimilhança. Os outros enfoques não produzem estimadores das covariâncias de forma tão direta. Além disso, sendo o modelo verdadeiro, é o estimador que produz menor variância assintótica. Na seção seguinte apresentamos o algoritmo EM para calcular os estimadores de máxima verossimilhança (EMV).

\section{Algoritmo EM}

Para implementar o algoritmo EM, consideramos a função de log-verossimillhança do modelo (6.6), pode ser escrita como

$$
\begin{aligned}
l(\boldsymbol{\theta}) \propto & -n \log \left(\sigma_{11} \sigma_{22}\right)-\frac{n}{2} \log \left(1-\rho_{12}^{2}\right)-\frac{1}{2\left(1-\rho_{12}^{2}\right) \sigma_{11}^{2}} \\
& \times \sum_{i=1}^{n}\left(y_{i}-\alpha-\beta \mu_{x}\right)^{2}+\frac{\rho_{12}}{\left(1-\rho_{12}^{2}\right) \sigma_{11} \sigma_{22}} \sum_{i=1}^{n}\left(y_{i}-\alpha-\beta \mu_{x}\right)\left(w_{i}-\mu_{x}\right) \\
& -\frac{1}{2\left(1-\rho_{12}^{2}\right) \sigma_{22}^{2}} \sum_{i=1}^{n}\left(w_{i}-\mu_{x}\right)^{2}
\end{aligned}
$$

onde

$\sigma_{11}=\left(\beta^{2} \sigma_{x}^{2}+\sigma_{\epsilon}^{2}\right)^{1 / 2}, \sigma_{22}=\left(\sigma_{x}^{2}+\sigma_{\xi}^{2}\right)^{1 / 2}, \sigma_{12}=\beta \sigma_{x}^{2}$ e $\rho_{12}=\frac{\beta \sigma_{x}^{2}}{\left(\beta^{2} \sigma_{x}^{2}+\sigma_{\epsilon}^{2}\right)^{1 / 2}\left(\sigma_{x}^{2}+\sigma_{\xi}^{2}\right)^{1 / 2}}$. Neste caso, podemos identificar dois conjunto de dados. O primeiro é composto pelas variáveis aleatórias $\left(Y_{i}, w_{i}, \gamma_{i}\right), i=1, \ldots, n$, que formam o conjunto de dados completos mas, inobserváveis (dada pela presença da variável inobservável ou latente $\gamma_{i}$ ) e o 
segundo, dado por $\left(Y_{i}, w_{i}\right), i=1, \ldots, n$ que formam o conjunto de dados incompletos e observáveis. Essa maneira de apresentar os dados segue o uso do algoritmo EM para a estimação dos parâmetros. Em nosso estudo as variáveis de censura formam a parte inobservável dos dados. Assim, a função de log-verossimilhança completa é dada por

$$
\begin{aligned}
l_{c}(\theta)= & -n \log \left(\sigma_{11} \sigma_{22}\right)-\frac{n}{2} \log \left(1-\rho_{12}^{2}\right)-\frac{1}{2\left(1-\rho_{12}^{2}\right) \sigma_{11}^{2}} \sum_{i=1}^{m}\left(y_{i}-\alpha-\beta \mu_{x}\right)^{2} \\
& -\frac{1}{2\left(1-\rho_{12}^{2}\right) \sigma_{11}^{2}} \sum_{i=m+1}^{n}\left(\gamma_{i}-\alpha-\beta \mu_{x}\right)^{2}+\frac{\rho_{12}}{\left(1-\rho_{12}^{2}\right) \sigma_{11} \sigma_{22}} \sum_{i=1}^{m}\left(y_{i}-\alpha-\beta \mu_{x}\right) \\
& \times\left(w_{i}-\mu_{x}\right)+\frac{\rho_{12}}{\left(1-\rho_{12}^{2}\right) \sigma_{11} \sigma_{22}} \sum_{i=m+1}^{n}\left(\gamma_{i}-\alpha-\beta \mu_{x}\right)\left(w_{i}-\mu_{x}\right) \\
& -\frac{1}{2\left(1-\rho_{12}^{2}\right) \sigma_{22}^{2}} \sum_{i=1}^{n}\left(w_{i}-\mu_{x}\right)^{2}+C,
\end{aligned}
$$

onde $C$ é uma constante que não depende dos parâmetros de interesse. As primeiras $m$ observações não têm censura e as $n-m$ observações restantes são censuradas. Para implementar o algoritmo EM, calculamos no passo E as estimativas de $\gamma_{i}$ para serem usadas no passo $\mathbf{M}$ do algoritmo. Logo, o processo iterativo resume-se aos seguintes passos:

Passo E: Calcular as esperanças condicionais da função de log-verossimilhança aumentada, ou seja, $\left.E\left(\gamma_{i} \mid \gamma_{i} \geq C_{i}, y_{i}, w_{i}, \boldsymbol{\theta}^{(i)}\right)\right)$ e $\left.E\left(\gamma_{i}^{2} \mid \gamma_{i} \geq C_{i}, y_{i}, w_{i}, \boldsymbol{\theta}^{(i)}\right)\right)$. Para o cálculo destas esperanças utilizamos o Lema A.4. Sabemos que a distribuição condicional de $y_{i}$ dada $x_{i}$ é tal que

$$
Y_{i} \mid x_{i} \sim N\left(\alpha+\beta x_{i} ; \sigma_{\epsilon}^{2}\right) \text {. }
$$

As esperanças condicionais utilizando o Lema A.4 são dadas por

$$
E\left(\gamma_{i} \mid \gamma_{i} \geq C_{i}, y_{i}, w_{i}, \theta^{(i)}=\mu_{c i}+\frac{\phi\left(\frac{c-\mu_{c i}}{\sigma_{c}}\right)}{1-\Phi\left(\frac{c-\mu_{c i}}{\sigma_{c}}\right)} \sigma_{c}\right.
$$


e

$$
E\left(\gamma_{i}^{2} \mid \gamma_{i} \geq C_{i}, y_{i}, w_{i}, \theta^{(i)}\right)=\mu_{c i}^{2}+\sigma_{c}^{2}+\frac{\phi\left(\frac{c-\mu_{c i}}{\sigma_{c}}\right)}{1-\Phi\left(\frac{c-\mu_{i}}{\sigma_{c}}\right)}\left(\mu_{c i}+c\right) \sigma_{c}
$$

onde $\mu_{c i}$ e $\sigma_{c}^{2}$ são dados anteriormente.

Passo M: Consiste em maximizar $Q\left(\boldsymbol{\theta}, \boldsymbol{\theta}^{(i)}\right)$ com respeito $\boldsymbol{\theta}$, onde

$$
\begin{aligned}
Q\left(\boldsymbol{\theta}, \theta^{(i)}\right)= & -n \log \left(\sigma_{11} \sigma_{22}\right)-\frac{n}{2} \log \left(1-\rho_{12}^{2}\right) \\
& -\frac{1}{2\left(1-\rho_{12}^{2}\right) \sigma_{11}^{2}} \sum_{j=1}^{m}\left(y_{j}-\alpha-\beta \mu_{x}\right)^{2} \\
& -\frac{1}{2\left(1-\rho_{12}^{2}\right) \sigma_{11}^{2}} \sum_{i=m+1}^{n}\left\{E\left(\gamma_{i}^{2} / \gamma_{i} \geq C_{i} ; \theta^{(i)}\right)\right. \\
& \left.-2\left(\alpha+\beta \mu_{x}\right) E\left(\gamma_{i} / S_{i} \geq C_{i} ; \theta^{(i)}\right)+\left(\alpha+\beta \mu_{x}\right)^{2}\right\} \\
& +\frac{\rho_{12}}{\left(1-\rho_{12}^{2}\right) \sigma_{11} \sigma_{22}} \sum_{j=1}^{m}\left(y_{j}-\alpha-\beta \mu_{x}\right)\left(w_{j}-\mu_{x}\right) \\
& +\frac{\rho_{12}}{\left(1-\rho_{12}^{2}\right) \sigma_{11} \sigma_{22}} \sum_{j=m+1}^{n}\left\{E\left(\gamma_{j} \mid \gamma_{j} \geq C_{j} ; \theta^{(j)}\right)-\left(\alpha+\beta \mu_{x}\right)\right\} \\
& \times\left(w_{j}-\mu_{x}\right)-\frac{1}{2\left(1-\rho_{12}^{2}\right) \sigma_{22}^{2}} \sum_{j=1}^{n}\left(w_{j}-\mu_{x}\right)^{2}+C .
\end{aligned}
$$

O cálculo das estimativas é feita através de maximização numérica, utilizando o mesmo processo apresentado para o método de máxima verossimilhança.

Existem vários critérios de convergência utilizadas na literatura. Dentre eles citamos apenas dois. Sejam $\boldsymbol{\theta}^{k}$ a estimativa de $\boldsymbol{\theta}$ na $k$-ésima iteração, $k=0,1,2, \ldots$ Como critério de convergência do algoritmo EM pode ser usado

$$
\left\|\theta^{(k+1)}-\theta^{(k)}\right\|<\epsilon,
$$

onde $\|c\|$ indica a norma do vetor $c$ e $\epsilon>0$, o alternativamente

$$
\frac{\left\|\theta^{(k+1)}-\theta^{(k)}\right\|}{\left\|\theta^{(k)}\right\|}<\epsilon
$$

Em nosso caso, declaramos convergência quando $\left\|\theta^{(j+1)}-\theta^{(j)}\right\|<e^{-6}$. 


\section{Método de regressão-calibração}

Um dos métodos mais usados para a correção do viés em modelos de regressão com erros de medidas é o chamado método de regressão-calibração. Este método discutido em Carroll e Stefanski (1990) pode ser usado para se obter estimadores aproximadamente consistentes. A base deste método é a troca da covariável não observada $x$ por um estimador da esperança condicional de $x$ dado $w$, ou seja, $E(x \mid w)$, e o uso de um procedimento padrão para ajustar o modelo com dados censurados. Carroll et al. (1995) relatam que este método produz estimadores aproximadamente consistentes para o coeficiente angular na maioria das aplicações de modelos lineares generalizados. Como uma forma de ilustração deste método, tomamos o modelo linear estrutural com erro aditivo, com média e variância dadas por

$$
E(Y \mid x)=\alpha+\beta x \text { e } \operatorname{Var}(Y \mid x)=\sigma_{\epsilon}^{2}
$$

Considere que $E(x \mid w)$ é estimada pela função $r(w, t)$, chamada função de calibração, que depende do vetor de parâmetros $t$. A troca de $x$ por seu estimador $r(w, t)$, estabelece um modelo modificado para os dados observados. Supondo que o erro de medida é não diferenciável (Bolfarine e Arellano-Valle, 1998), ou seja, que a distribuição de $Y$ dado $r(w, t)$ depende apenas de $x$, segundo Carroll et al. (1995), o método de regressão-calibração, neste caso reproduz precisamente a função de regressão dada por

$$
E(Y \mid x)=\alpha+\beta r(w, t)
$$

A variância dos dados observados é maior para este modelo, ou seja,

$$
\operatorname{Var}(Y \mid x)=\sigma_{\epsilon}^{2}+\beta^{2} \operatorname{Var}(x \mid w)
$$


Para nosso trabalho, consideramos um algoritmo que produz uma aproximação linear para a função de calibração, aplicável quando $\sigma_{\xi}^{2}$ é conhecido. A estimação da função de calibração tem como base a teoria para obtenção da melhor aproximação linear de uma variável aleatória $x$, condicionadas à variável $\bar{w}$, onde $\bar{w}=\sum_{i=1}^{n} \frac{w_{i}}{n}$ e $\widehat{\sigma_{x}^{2}}=$ $\sum_{i=1}^{n} \frac{\left(w_{j}-\bar{w}\right)^{2}}{n-1}$. Assim, a estimativa da função de calibração proposta por este método é dada por

$$
E\left(x_{j} \mid w_{j}\right)=\mu_{x}+\frac{\sigma_{x}^{2}}{\sigma_{x}^{2}+\sigma_{\xi}^{2}}\left(w_{j}-\mu_{x}\right) \text { e } \operatorname{Var}\left(x_{j} \mid w_{j}\right)=\frac{\sigma_{x}^{2} \sigma_{\xi}^{2}}{\sigma_{x}^{2}+\sigma_{\xi}^{2}} .
$$

Quando desconhecidos, $\mu_{x}$ e $\sigma_{x}^{2}$ são substituídos por seus estimadores.

\section{Método de simulação e extrapolação (SIMEX)}

O método de simulação e extrapolação (SIMEX) é definido como um método de estimação e redução do viés devido ao erro de medida, baseado em simulação, que não faz nenhuma suposição a respeito da distribuição da covariável não observada x. Esta técnica proposta por Cook e Stefanski (1994) é aplicável a métodos gerais de estimação na presença de erros de medição. Carroll et al. (1995) apresentam com mais detalhes este método. Estes autores argumentam que o SIMEX representa um método que reproduz réplicas da variável explicativa da mesma forma que o método bootstrap reproduz réplicas da amostra.

\section{Procedimento para a estimação de $\theta$}

Consideramos o modelo (6.1) e (6.2) para descrever este procedimento. O algoritmo é composto de duas etapas: simulação e extrapolação.

\section{Etapa de simulação}

1. fixar B suficientemente grande, 
2. fixar $\lambda_{1} \in(0,2]$,

3. para cada $b=1, \ldots, B$ :

3.1. gerar $J_{b, i}$ com distribuição $N\left(0, \sigma_{\xi}^{2}\right), i=1, \ldots, n$, todos independentes e calcular

$$
w_{b, i}=w_{i}+\lambda_{1}^{1 / 2} J_{b, i}
$$

3.2. usando algoritmos usuais de estimação para dados censurados, obter as estimativas ingênuas $\hat{\theta}_{b}\left(\lambda_{1}\right)$, substituindo $x_{i}$ por $w_{b, i}, i=1, \ldots, n$,

4. calcular

$$
\hat{\boldsymbol{\theta}}\left(\lambda_{1}\right)=\frac{1}{B} \sum_{b=1}^{B} \hat{\boldsymbol{\theta}}_{b}\left(\lambda_{1}\right)
$$

$\mathrm{e}$

5. repetir os passos (2) - (4) para $s=1, \ldots, M$, com $\lambda_{s} \in(0,2]$, com $M$ inteiro arbitrário, para nosso caso consideramos $M=100$.

\section{Etapa de extrapolação}

6. Relacionar $\hat{\boldsymbol{\theta}}(\boldsymbol{\lambda})$ e $\boldsymbol{\lambda}=\left(0, \lambda_{1}, \ldots, \lambda_{M}\right)$, onde $\hat{\boldsymbol{\theta}}(0)$ é a estimativa ingênua através de um modelo de regressão geralmente quadrático.

7. Fazer $\lambda=-1$ na regressão estimada, obtendo desta forma o estimador $\widehat{\boldsymbol{\theta}}_{\text {SIMEX }}$. Ver Valença (2003) para detalhes da estimação de variância de $\widehat{\boldsymbol{\theta}}_{\text {SIMEX }}$.

\section{Comparação entre os métodos de estimação}

Realizamos agora um estudo de simulação a fim de investigar o desempenho dos métodos de estimação descritos neste capítulo. Desejamos basicamente avaliar as mudanças no comportamento dos diferentes estimadores, considerando três aspectos: nível de censura existente, tamanho da amostra e o aumento da variância do erro de medida. Os dados foram gerados de acordo com o modelo (6.1) e (6.2) para a 
distribuição log-normal. A variáveis resposta $Y_{i}$ foram geradas com valores extraídos de uma $N(1,1)$. Os valores da covariável $x_{i}$ foram amostrados de uma distribuição $N\left(\mu_{x}, \sigma_{x}^{2}\right)$. Os erros $\epsilon_{i}$ foram gerados independente e identicamente distribuídos (iid) com distribuição $N(0,1)$ e erros de medida $\xi_{i}$ foram gerados de uma distribuição $N\left(0, \sigma_{\xi}^{2}\right)$ independentes e identicamente distribuídos. Os verdadeiros valores para os coeficientes do modelo foram $\alpha=0.5, \beta=1$ e $\sigma_{x}=1$. Os tempos de censura $C_{i}$ foram gerados como variáveis independentes com distribuição uniforme $(0,1)$. As porcentagens de censura consideradas foram $0 \%$ (sem censura), 30\%, 50\% e 80\%. Para obter os estimadores SIMEX usamos, na etapa da extrapolação, funções quadráticas para todos os parâmetros.

Investigamos os resultados para três tamanhos de amostras: 50, 250 e 500. Sob cada combinação de parâmetros, realizamos 500 réplicas. As simulações foram implementadas na linguagem de programação $R$, sendo a maximização da função de verossimilhança obtida da função de máximização optim, através do método LBFGS-B, descrito no método de máxima verossimilhança.

\section{Resultados}

As tabelas que seguem resumem parte dos resultados das simulações para dois valores da variância do erro de medida $\left(\sigma_{\xi}^{2}=0.3\right.$ e $\left.\sigma_{\xi}^{2}=0.5\right)$, e permitem comparar valores estimados com diferentes níveis de cesura, para amostras de tamanho 50 (Tabelas 6.1 e 6.3) e 250 (Tabelas 6.2 e 6.4).

Notamos que em geral o método de máxima verossimlhança e regressão-calibração parece apresentar boas estimativas para todos os parâmetros levando em conta a média. No entanto, o método ingênuo não apresenta boas estimativas para todos os parâmetros, mas parece ser mais preciso em relação aos outros métodos, por apre- 
sentar menor erro padrão.

Nas Tabelas 6.1 e 6.3 verificamos que a medida que aumenta a variância do erro de medida, aumenta os valores das estimativas dos parãmetros, em quase todos os métodos de estimação.

Notamos que o aumento do tamanho da amostra (Tabela 6.1 e 6.2) se aproxima mais do valor da estimativa dos parâmetros. As oscilações das estimativas dos parâmetros em relação ao aumento da proporção de censura aparentemente são mais visíveis em amostras menores (Tabela 6.1). Em todas as tabelas, notamos que o erro padrão aumenta com o aumento da proporção de censura.

Um problema com o método de regressão-calibração é a estimação da variância. Existem apenas aproximações (Carroll et al., 1995). 
Tabela 6.1: Média das estimativas de Monte Carlo dos parâmetros e correspondente erro padrão, com variância do erro de medida $\sigma_{\xi}^{2}=0,3$ e tamanho de amostra $n=50$.

Verdadeiros valores: $\alpha=0,5 ; \beta=1,0 ; \mu_{x}=1,0 ; \sigma_{x}^{2}=1,0$ e $\sigma_{\epsilon}^{2}=0,5$

\begin{tabular}{|c|c|c|c|c|c|}
\hline Censura & Parâmetro & $\begin{array}{c}\text { Máxima } \\
\text { Verossimilhança }\end{array}$ & SIMEX & $\begin{array}{l}\text { Regressão } \\
\text { Calibração }\end{array}$ & Ingênuo \\
\hline \multirow{5}{*}{$0 \%$} & $\alpha$ & $0,46620(0,21458)$ & $0,53437(0,19095)$ & $0,50017(0,21530)$ & $0,72325(0,16118)$ \\
\hline & $\beta$ & $1,02823(0,16312)$ & $0,96962(0,14222)$ & $1,00777(0,16734)$ & $0,77223(0,10963)$ \\
\hline & $\mu_{x}$ & $1,00159(0,16312)$ & $1,00696(0,14222)$ & $0,98836(0,16734)$ & $1,00696(0,10963)$ \\
\hline & $\sigma_{x}^{2}$ & $0,97101(0,25282)$ & $1,67419(0,36622)$ & $0,92728(0,26408)$ & $1,97419(0,41006)$ \\
\hline & $\sigma_{\epsilon}^{2}$ & $0,46679(0,16687)$ & $0,74359(0,09951)$ & $0,82758(0,08267)$ & $0,83772(0,08935)$ \\
\hline & $\alpha$ & $0,72542(0,24655)$ & $0,74299(0,22784)$ & $0,72753(0,24792)$ & $0,98033(0,19218)$ \\
\hline & $\beta$ & $1,02989(0,18265)$ & $0,96001(0,17470)$ & $1,01110(0,20502)$ & $0,77407(0,12383)$ \\
\hline \multirow[t]{5}{*}{$30 \%$} & $\mu_{x}$ & $0,99348(0,16221)$ & $1,00696(0,09951)$ & $0,98836(0,08267)$ & $1,00696(0,08935)$ \\
\hline & $\sigma_{x}^{2}$ & $0,97239(0,24621)$ & $1,67419(0,09951)$ & $0,92728(0,08267)$ & $1,97419(0,08935)$ \\
\hline & $\sigma_{\epsilon}^{2}$ & $0,57425(0,20668)$ & $0,74359(0,09951)$ & $0,82758(0,08267)$ & $0,83772(0,08935)$ \\
\hline & $\alpha$ & $0,94478(0,29303)$ & $0,93087(0,28711)$ & $0,99440(0,27223)$ & $1,20441(0,23316)$ \\
\hline & $\beta$ & $1,03132(0,22383)$ & $0,95397(0,18060)$ & $1,01516(0,22102)$ & $0,77475(0,15870)$ \\
\hline \multirow[t]{5}{*}{$50 \%$} & $\mu_{x}$ & $1,00767(0,24655)$ & $1,00696(0,09951)$ & $0,98836(0,08267)$ & $1,00696(0,08935)$ \\
\hline & $\sigma_{x}^{2}$ & $0,96811(0,24655)$ & $1,67419(0,09951)$ & $0,92728(0,08267)$ & $1,97419(0,08935)$ \\
\hline & $\sigma_{\epsilon}^{2}$ & $0,65982(0,28390)$ & $0,80703(0,14109)$ & $0,93794(0,16718)$ & $0,94332(0,14015)$ \\
\hline & $\alpha$ & $1,59312(0,48209)$ & $1,48173(0,59491)$ & $1,59334(0,39120)$ & $1,84219(0,41875)$ \\
\hline & $\beta$ & $1,03212(0,33433)$ & $0,95472(0,24633)$ & $1,02538(0,30173)$ & $0,77272(0,24035)$ \\
\hline \multirow[t]{3}{*}{$80 \%$} & $\mu_{x}$ & $0,99763(0,16586)$ & $1,00696(0,09951)$ & $0,98836(0,08267)$ & $1,00696(0,08935)$ \\
\hline & $\sigma_{x}^{2}$ & $0,99506(0,25482)$ & $1,67419(0,09951)$ & $0,92728(0,08267)$ & $1,97419(0,08935)$ \\
\hline & $\sigma_{\epsilon}^{2}$ & $0,89087(0,56492)$ & $0,80703(0,32774)$ & $0,93794(0,24974)$ & $0,94332(0,24755)$ \\
\hline
\end{tabular}


Tabela 6.2: Média das estimativas de Monte Carlo dos parâmetros e correspondente erro padrão, com variância do erro de medida $\sigma_{\xi}^{2}=0,3$ e tamanho de amostra $n=250$. Verdadeiros valores: $\alpha=0,5 ; \beta=1,0 ; \mu_{x}=1,0 ; \sigma_{x}^{2}=1,0$ e $\sigma_{\epsilon}^{2}=0,5$

\begin{tabular}{cccccc}
\hline \hline Censura & Parâmetro & Máxima & SIMEX & Regressão & Ingênuo \\
& \multicolumn{5}{c}{ Verossimilhança } \\
\hline \multirow{2}{*}{$0 \%$} & $\alpha$ & $0,50013(0,08860)$ & $0,54204(0,08288)$ & $0,50209(0,08288)$ & $0,72547(0,07104)$ \\
& $\beta$ & $1,00435(0,06593)$ & $0,95342(0,05825)$ & $0,99569(0,07309)$ & $0,76834(0,04771)$ \\
& $\mu_{x}$ & $0,99709(0,07135)$ & $1,00372(0,14222)$ & $1,00058(0,16734)$ & $1,00371(0,10963)$ \\
& $\sigma_{x}^{2}$ & $0,98869(0,10795)$ & $1,69753(0,36622)$ & $0,23081(0,26408)$ & $1,99753(0,41006)$ \\
& $\sigma_{\epsilon}^{2}$ & $0,49579(0,05042)$ & $0,74663(0,03059)$ & $0,85033(0,02728)$ & $0,85235(0,02650)$ \\
\hline \multirow{3}{*}{$30 \%$} & $\alpha$ & $0,74700(0,09813)$ & $0,77771(0,09932)$ & $0,74957(0,11107)$ & $0,98268(0,08064)$ \\
& $\beta$ & $1,00469(0,07563)$ & $0,94893(0,17470)$ & $1,01110(0,20502)$ & $0,77461(0,05328)$ \\
& $\mu_{x}$ & $0,99559(0,07414)$ & $1,00371(0,09951)$ & $1,00058(0,08267)$ & $1,00371(0,08935)$ \\
& $\sigma_{x}^{2}$ & $0,99563(0,11149)$ & $1,69753(0,09951)$ & $0,23081(0,08267)$ & $1,99753(0,08935)$ \\
& $\sigma_{\epsilon}^{2}$ & $0,62063(0,07011)$ & $0,79702(0,04330)$ & $0,92148(0,03319)$ & $0,91588(0,03769)$ \\
\hline \hline \multirow{2}{*}{$50 \%$} & $\alpha$ & $0,99690(0,11712)$ & $0,95180(0,11456)$ & $0,96035(0,11221)$ & $1,20259(0,09909)$ \\
& $\beta$ & $1,00499(0,08423)$ & $0,95638(0,07387)$ & $1,02014(0,08308)$ & $0,76608(0,06047)$ \\
& $\mu_{x}$ & $1,00106(0,07388)$ & $1,00371(0,09951)$ & $1,00058(0,08267)$ & $1,00371(0,08935)$ \\
& $\mu_{x}^{2}$ & $1,00106(0,11146)$ & $1,69753(0,09951)$ & $0,23081(0,08267)$ & $1,99753(0,08935)$ \\
& $\sigma_{x}^{2}$ & $0,99999(0,07071)$ & $1,00371(0,09951)$ & $1,00058(0,08267)$ & $1,00371(0,08935)$ \\
& $\sigma_{\epsilon}^{2}$ & $0,70119(0,08729)$ & $0,81999(0,07662)$ & $0,96251(0,04402)$ & $0,96508(0,04262)$ \\
\hline
\end{tabular}


Tabela 6.3: Média das estimativas de Monte Carlo dos parâmetros e correspondente erro padrão, com variância do erro de medida $\sigma_{\xi}^{2}=0,5$ e tamanho de amostra $n=50$. Verdadeiros valores: $\alpha=0,5 ; \beta=1,0 ; \mu_{x}=1,0 ; \sigma_{x}^{2}=1,0$ e $\sigma_{\epsilon}^{2}=0,5$

\begin{tabular}{cccccc}
\hline \hline Censura & Parâmetro & Máxima & SIMEX & $\begin{array}{c}\text { Regressão } \\
\text { Calibração }\end{array}$ & Ingênuo \\
& \multicolumn{5}{c}{ Verossimilhança } \\
\hline \multirow{2}{*}{$0 \%$} & $\alpha$ & $0,46085(0,22102)$ & $0,63967(0,18039)$ & $0,48478(0,24165)$ & $0,85314(0,15140)$ \\
& $\beta$ & $1,03713(0,19465)$ & $0,91667(0,14026)$ & $1,04141(0,18881)$ & $0,65309(0,09696)$ \\
& $\mu_{x}$ & $1,00236(0,16124)$ & $0,97548(0,20787)$ & $1,00846(0,17249)$ & $0,97548(0,20787)$ \\
& $\sigma_{x}^{2}$ & $0,96934(0,30912)$ & $1,44428(0,43879)$ & $0,33296(0,36595)$ & $1,94428(0,53789)$ \\
& $\sigma_{\epsilon}^{2}$ & $0,44221(0,17811)$ & $0,76089(0,10708)$ & $0,89183(0,08998)$ & $0,87693(0,09009)$ \\
\hline \multirow{3}{*}{$30 \%$} & $\alpha$ & $0,72728(0,29224)$ & $0,81544(0,20503)$ & $0,76007(0,27023)$ & $1,09283(0,17727)$ \\
& $\beta$ & $1,03972(0,23373)$ & $0,90956(0,15033)$ & $1,04854(0,22687)$ & $0,68818(0,10867)$ \\
& $\mu_{x}$ & $1,00236(0,16124)$ & $0,97548(0,20787)$ & $1,00846(0,17249)$ & $0,97548(0,20787)$ \\
& $\sigma_{x}^{2}$ & $0,96934(0,30912)$ & $1,44428(0,43879)$ & $0,33296(0,36595)$ & $1,94428(0,53789)$ \\
& $\sigma_{\epsilon}^{2}$ & $0,56156(0,24684)$ & $0,82791(0,13986)$ & $0,95485(0,11879)$ & $0,96674(0,12039)$ \\
\hline \hline \multirow{2}{*}{$50 \%$} & $\alpha$ & $0,96394(0,32646)$ & $1,05672(0,25759)$ & $0,97700(0,32021)$ & $1,37461(0,22263)$ \\
& $\beta$ & $1,04022(0,26274)$ & $0,89584(0,17910)$ & $1,06596(0,28811)$ & $0,66385(0,13331)$ \\
& $\mu_{x}$ & $1,00236(0,16124)$ & $0,97548(0,20787)$ & $1,00846(0,17249)$ & $0,97548(0,20787)$ \\
& $\sigma_{x}^{2}$ & $0,96934(0,30912)$ & $1,44428(0,43879)$ & $0,33296(0,36595)$ & $1,94428(0,53789)$ \\
& $\mu_{\epsilon}$ & $0,66591(0,30749)$ & $0,88396(0,16176)$ & $0,98443(0,15383)$ & $1,02279(0,13576)$ \\
\hline
\end{tabular}


Tabela 6.4: Média das estimativas de Monte Carlo dos parâmetros e correspondente erro padrão com variância do erro de medida $\sigma_{\xi}^{2}=0,5$ e tamanho de amostra $n=250$.

Verdadeiros valores: $\alpha=0,5 ; \beta=1,0 ; \mu_{x}=1,0 ; \sigma_{x}^{2}=1,0$ e $\sigma_{\epsilon}^{2}=0,5$

\begin{tabular}{cccccc}
\hline \hline Censura & Parâmetro & Máxima & SIMEX & $\begin{array}{c}\text { Regressão } \\
\text { Calibração }\end{array}$ & Ingênuo \\
& \multicolumn{5}{c}{ Verossimilhança } \\
\hline \multirow{2}{*}{$0 \%$} & $\alpha$ & $0,48827(0,10415)$ & $0,61033(0,08617)$ & $0,48834(0,11230)$ & $0,83314(0,07397)$ \\
& $\beta$ & $1,01374(0,07814)$ & $0,89072(0,05696)$ & $1,00898(0,09488)$ & $0,66912(0,04214)$ \\
& $\mu_{x}$ & $0,99332(0,07686)$ & $1,01447(0,09498)$ & $1,00769(0,07908)$ & $1,01447(0,09498)$ \\
& $\sigma_{x}^{2}$ & $0,98274(0,07587)$ & $1,50023(0,18044)$ & $0,33216(0,14501)$ & $2,00023(0,38143)$ \\
& $\sigma_{\epsilon}^{2}$ & $0,48109(0,08449)$ & $0,78654(0,04724)$ & $0,90158(0,04439)$ & $0,90716(0,04049)$ \\
\hline \multirow{3}{*}{$30 \%$} & $\alpha$ & $0,75744(0,11674)$ & $0,86175(0,10661)$ & $0,75936(0,12583)$ & $1,12598(0,09211)$ \\
& $\beta$ & $1,01877(0,09713)$ & $0,88447(0,07101)$ & $1,01323(0,09978)$ & $0,66257(0,05248)$ \\
& $\mu_{x}$ & $0,99332(0,07686)$ & $1,01447(0,09498)$ & $1,00769(0,07908)$ & $1,01447(0,09498)$ \\
& $\sigma_{x}^{2}$ & $0,98274(0,07587)$ & $1,50023(0,18044)$ & $0,33216(0,14501)$ & $2,00023(0,38143)$ \\
& $\sigma_{\epsilon}^{2}$ & $0,63668(0,12783)$ & $0,84714(0,06289)$ & $0,97757(0,04928)$ & $0,97765(0,05336)$ \\
\hline \hline \multirow{2}{*}{$50 \%$} & $\alpha$ & $0,99936(0,13029)$ & $1,02358(0,11661)$ & $0,99748(0,12121)$ & $1,32645(0,09653)$ \\
& $\beta$ & $1,02948(0,09989)$ & $0,88937(0,07463)$ & $1,01802(0,09696)$ & $0,67506(0,05477)$ \\
& $\mu_{x}$ & $0,99332(0,07686)$ & $1,01447(0,09498)$ & $1,00769(0,07908)$ & $1,01447(0,09498)$ \\
& $\mu_{x}^{2}$ & $0,98274(0,07587)$ & $1,50023(0,18044)$ & $0,33216(0,14501)$ & $2,00023(0,38143)$ \\
& $\sigma_{x}^{2}$ & $0,98274(0,07587)$ & $1,50023(0,18044)$ & $0,33216(0,14501)$ & $2,00023(0,38143)$ \\
& $\sigma_{\epsilon}^{2}$ & $0,74354(0,15852)$ & $0,81999(0,07268)$ & $1,03256(0,05844)$ & $0,94971(0,06599)$ \\
\hline
\end{tabular}




\subsubsection{Estimação da função de sobrevivência}

Em uma análise de um modelo paramétrico de sobrevivência existe interesse em estimar a curva de sobrevivência do modelo. Desta forma investigamos os ajustes obtidos pelos diferentes métodos, na presença do erro de medida, com base na curva teórica da distribuição normal. Note que

$$
\begin{aligned}
S\left(t_{i}\right) & =P\left(T_{i}>t_{i}\right)=P\left(Y_{i}>y_{i}\right)=E\left[P\left(Y_{i}>y_{i} \mid x_{i}\right)\right] \\
& =\int_{-\infty}^{+\infty} P\left(Y_{i}>y_{i} \mid x_{i}\right) f\left(x_{i}\right) d x_{i},
\end{aligned}
$$

$i=1, \ldots, n$. De acordo com (6.3) e (6.5),

$$
Y_{i} \mid x_{i} \sim N\left(\alpha+\beta x_{i} ; \sigma_{\epsilon}^{2}\right)
$$

$\mathrm{e}$

$$
x_{i} \sim N\left(\mu_{x} ; \sigma_{x}^{2}\right)
$$

Utilizando o Lema A.4 para a distribuição de $Y_{i} \mid x_{i}$, e padronizando $x_{i}$, temos que

$$
z_{i}=\frac{x_{i}-\mu_{x}}{\sigma_{x}}
$$

Logo,

$$
Y_{i} \mid z_{i} \sim N\left(\alpha+\beta \mu_{x}+\beta \sigma_{x} z_{i} ; \sigma_{\epsilon}^{2}\right) \text {. }
$$

Expressando na forma do Lema A.4, temos que

$g=\alpha+\beta \mu_{x}$ e $h=\beta \sigma_{x}$.

Assim,

$$
Y_{i} \mid z_{i} \sim N\left(g+h z_{i} ; \sigma_{\epsilon}^{2}\right)
$$


Logo,

$$
\begin{aligned}
P\left(Y_{i}>y_{i} \mid z_{i}\right) & =\int_{y_{i}}^{+\infty} \frac{1}{\sqrt{2 \pi} \sigma_{\epsilon}} \phi\left(\frac{t_{i}-g-h z_{i}}{\sigma_{\epsilon}}\right) d t_{i} \\
E\left[P\left(Y_{i}>y_{i} \mid z_{i}\right)\right] & =\int_{-\infty}^{+\infty} \int_{y_{i}}^{+\infty} \frac{1}{\sqrt{2 \pi} \sigma_{\epsilon}} \phi\left(\frac{t_{i}-g-h z_{i}}{\sigma_{\epsilon}}\right) d t_{i} \phi\left(z_{i}\right) d z_{i} .
\end{aligned}
$$

Fazendo mudança de variável e calculando as integrais, temos

$$
E\left[P\left(Y_{i}>y_{i} \mid z_{i}\right)\right]=1-\Phi\left\{\frac{y_{i}-\alpha-\beta \mu_{x}}{\sqrt{\sigma_{\epsilon}^{2}+\beta^{2} \sigma_{x}^{2}}}\right\} .
$$

Portanto, desde que $\widehat{\alpha}, \widehat{\beta}, \widehat{\mu_{x}}, \widehat{\sigma_{x}^{2}}$ e $\widehat{\sigma_{\epsilon}^{2}}$ sejam os EMV's respectivos, a função de sobrevivência é dado por

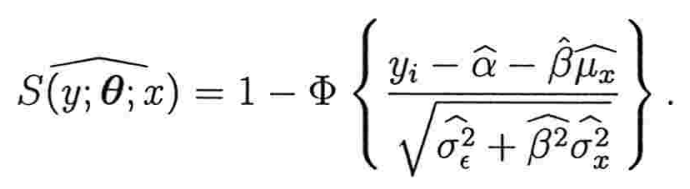

Realizamos algumas simulações para investigar o desempenho da curva de sobrevivência obtida pelos diferentes métodos de estimação, considerando valores diferentes para a variância do erro de medida. Fixando o vetor de parâmetros verdadeiros $\boldsymbol{\theta}_{0}=(0,5,1,0,1,0,1,0,0,5)$ na simulação, comparamos a curva teórica $S\left(y ; \boldsymbol{\theta}_{0}\right)$, com a curva estimada para cada método estudado, isto é, máxima verossimilhança, SIMEX, regressão-calibração e ingênuo. Assim, ajustamos respectivamente as curvas $\left.S_{M V}\left(y ; \boldsymbol{\theta}\left(\sigma_{\xi}^{2}\right)\right)\right), S_{S I M E X}\left(y ;\left(\sigma_{\xi}^{2}\right)\right), S_{R-C}\left(y ;\left(\sigma_{\xi}^{2}\right)\right)$ e $S_{I N G E N U A}\left(y ;\left(\sigma_{\xi}^{2}\right)\right)$, com variâncias do erro de medida iguais a 0.3 e 0.5 . As estimativas consideradas para $\boldsymbol{\theta}$ são as médias da réplicas para cada método e para cada valor de $\sigma_{\xi}^{2}$. Selecionamos dois tamanhos de amostra diferentes $(n=50$ e $n=250)$ e dois níveis de censura $0 \%$ e $30 \%$.

Na Figura 6.1 com nível de censura $0 \%$ e tamanho de amostra $n=50$, notamos que as curvas estimadas pelos métodos de máxima verossimilhança e regressão-calibração praticamente não diferem da curva teórica, enquanto os outros dois métodos diferem 
muito pouco. Notamos que os ajustes fornecidos pelos quatro métodos para o nível de censura $30 \%$ e tamanho de amostra $n=50$, apresenta comportamento parecido e todos diferem da curva teórica (Figura 6.2). 
Figura 6.1: Curvas de sobrevivência teórica e ajustadas, com base nas médias das estimativas dos parâmetros, tamanho de amostra 50 e $0 \%$ de censura, com erro de medida. Resultados para diferentes valores da variância do erro de medida $\left(\sigma_{\xi}^{2}\right)$. (a) Método de máxima verossimilhança, (b) Método SIMEX, (c) Método regressãocalibração e (d) Método ingênuo.

(a)

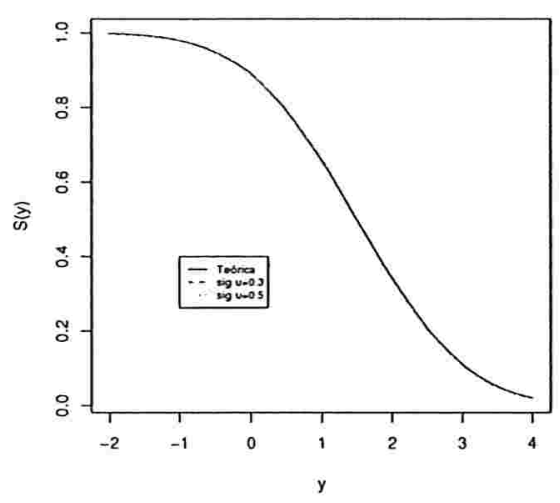

(c)

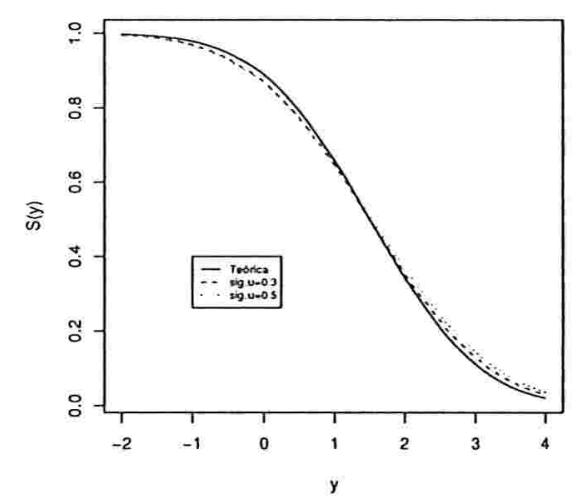

(b)

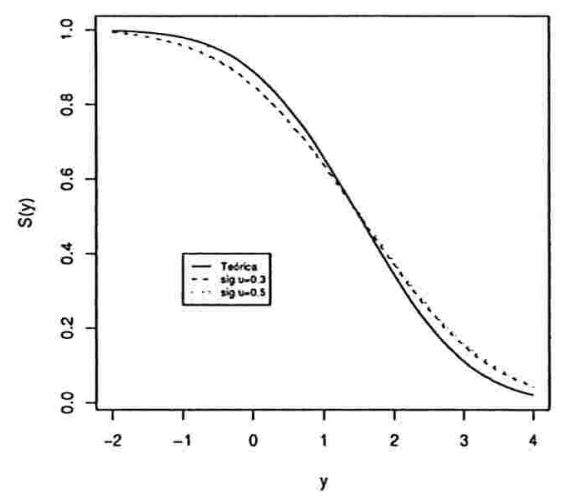

(d)

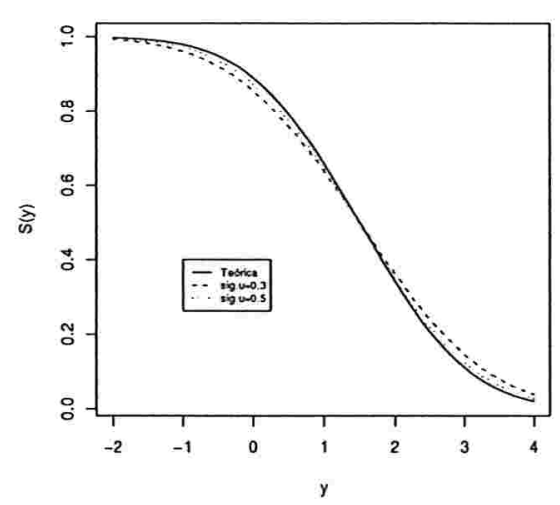


Figura 6.2: Curvas de sobrevivência teórica e ajustada, com base nas médias das estimativas dos parâmetros, tamanho de amostra 50 e $30 \%$ de censura, com erro de medida. Resultados para diferentes valores da variância do erro de medida $\left(\sigma_{\xi}^{2}\right)$. (a) Método de máxima verossimilhança, (b) Método SIMEX, (c) Método regressãocalibração e (d) Método ingênuo.

(a)

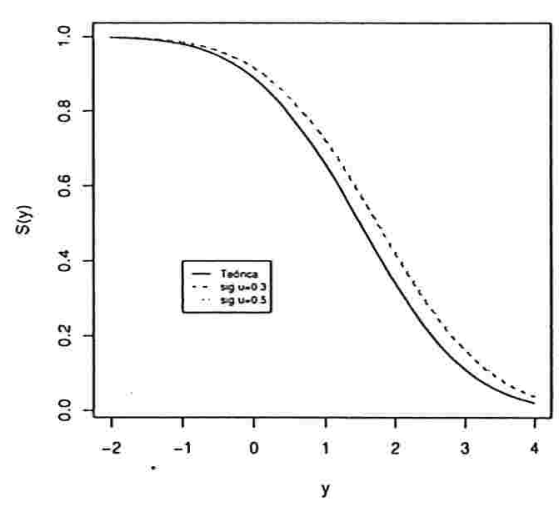

(c)

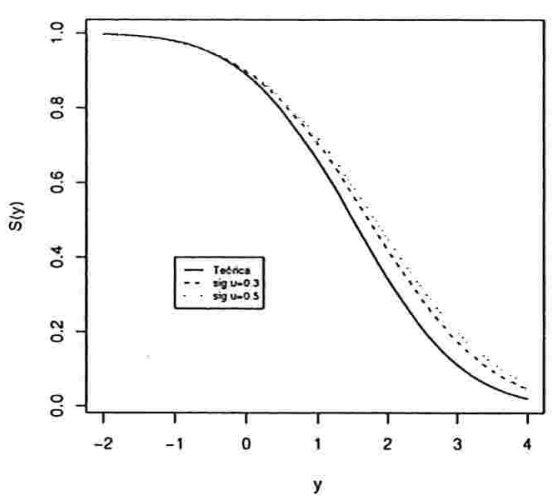

(b)

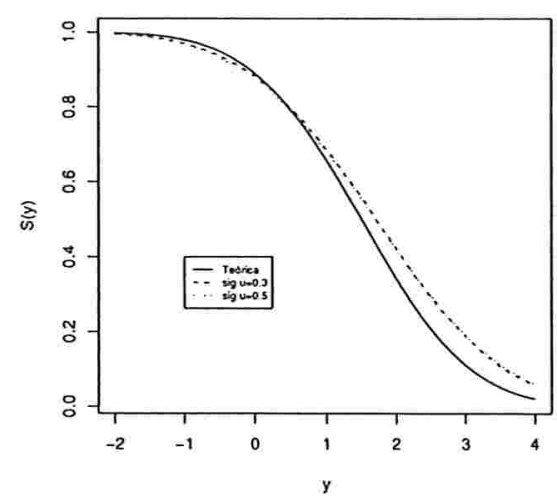

(d)

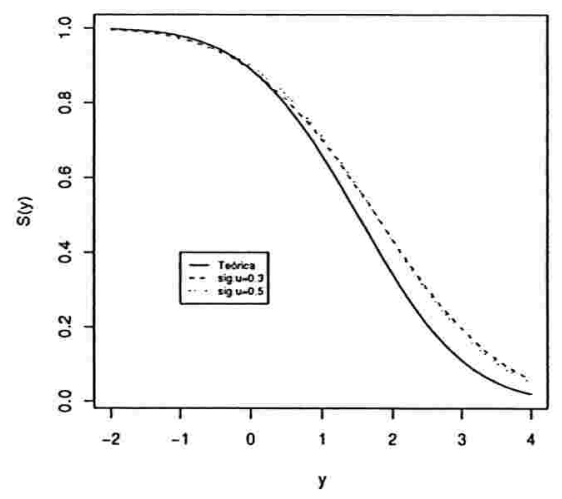




\subsection{Modelo com efeito aleatório}

Considere uma amostra com uma determinada estrutura de grupos para os dados, na qual $T_{i j}$ representa o tempo de vida (ou de falha) do indivíduo $i$ no grupo $j$, com $i=1, \ldots, n_{j}$ e $j=1, \ldots, m$. Seja $x_{i j}$ uma covariável (escalar) que não pode ser observada diretamente, mas em seu lugar observamos $w_{i j}$. O modelo log-linear para $Z_{i j}=\log T_{i j}$ contendo simultaneamente intercepto aleatório e erro de medida pode ser representado por

$$
\log T_{i j}=b_{j}+\beta x_{i j}+\epsilon_{i j}
$$

sendo $\epsilon_{i j}$ erros aleatórios independentes e identicamente distribuídos com o parâmetro $\beta$ desconhecido. Este modelo pode ser generalizado para situações mais gerais, onde consideramos $b_{i j}=S_{i j} b_{j}$ onde $b_{j}$ é o efeito aleatório e $S_{i j}$ é medida sem (ou com) erro. Existem sugestões na literatura comó, por exemplo, em Cuit et al. (2004), onde os autores consideram $S_{i j}$ medida com erro. Para nosso estudo, consideramos um modelo com erro de medida aditivo onde, ao invés de $x_{i j}$, observamos

$$
w_{i j}=x_{i j}+\xi_{i j}
$$

$\operatorname{com} \xi_{i j}$ representando os erros de medida aleatórios, independentes e identicamente distribuídas, conforme uma distribuição normal de média zero e variância $\sigma_{\xi}^{2}$ assumimos que $\sigma_{\xi}^{2}$ é conhecida devido à falta de identificabilidade. Em nosso estudo assumimos um modelo estrutural, onde é natural assumir que $x_{i j}$, $\xi_{i j}$ e aepsilon $n_{i j}$ sejam independentes, $x_{i j}$ segue uma distribuição normal de média $\mu_{x}$ e variância $\sigma_{x}^{2}$. De modo geral, para dados agrupados, poderíamos admitir correlção intragrupo para $x_{i j}$. Neste caso, considera-se um modelo linear misto para dados não observados $x_{i j}$ (Laird e Ware, 1982). O efeito aleatório do grupo $j$ é representado por $b_{j}=\alpha+\sigma_{b}^{2} V_{j}$, sendo $V_{j}$ 's variáveis aleatórias independentes e identicamente distribuídas segundo 
uma distribuição normal de média zero e variância um (Klein et al., 1999). Assumimos também que $b_{j}, e_{i j}$, e $x_{i j}$ são independentes entre si e que, condicionado no efeito aleatório $b_{j}$, as respostas em um grupo $j$ são independentes. Admitimos que os tempos de sobrevivência estão sujeitos a censura à direita, assumimos que a censura é aleatória e não informativa, ou seja, a sua distribuição não depende de parâmetros de interesse. Consideramos também $Y_{i j}=\min \left(\log T_{i j}, \log C_{i j}\right)$, e o indicador de vida (ou de falha) $\delta_{i j}$ definido como $\delta_{i j}=I\left(T_{i, j} \leq C_{i j}\right)$, sendo $I($.$) a função indicadora. Assim,$ os dados são representados pelos pares de variáveis aleatórias $\left(Y_{i j}, \delta_{i j}\right)$ e a covariável $w_{i j}, i=1, \ldots, n_{j}$ e $j=1, \ldots, k$. O modelo definido acima pode ser expresso como

$$
\begin{gathered}
Y_{i j} \mid x_{i, j} b_{j} \sim N\left(b_{j}+\beta x_{i j}, \sigma_{\epsilon}^{2}\right), \\
w_{i j} \mid x_{i j}, b_{j} \sim N\left(x_{i j}, \sigma_{\xi}^{2}\right), \\
x_{i j} \sim N\left(\mu_{x} ; \sigma_{x}^{2}\right),
\end{gathered}
$$

$\mathrm{e}$

$$
b_{j} \sim N\left(\alpha ; \sigma_{b}^{2}\right)
$$

Para se obter a densidade conjunta de $\left(Y_{i j}, w_{i j}\right)$, que é a amostra observada, temos que eliminar $x_{i j}, i=1, \ldots, n_{j}$ e $j=1, \ldots, m$, integrando em $x_{i j}$, como mostramos a seguir. A função de densidade conjunta de $\left(Y_{i j}, w_{i j}\right)$ para observações não censuradas pode ser expressa como

$$
\begin{aligned}
f\left(y_{i j}, w_{i j}\right) & =\int_{-\infty}^{+\infty} \int_{-\infty}^{+\infty} f\left(y_{i j}, w_{i j}, x_{i j}, b_{j}\right) d x_{i j} d b_{j} \\
& =\int_{-\infty}^{+\infty} \int_{-\infty}^{+\infty} f\left(y_{i j} \mid w_{i j}, x_{i j}, b_{j}\right) f\left(w_{i j} \mid x_{i j}, b_{j}\right) f\left(x_{i j}\right) f\left(b_{j}\right) d x_{i j} d b_{j} \\
& =\int_{-\infty}^{+\infty} f\left(b_{j}\right) \int_{-\infty}^{+\infty} f\left(y_{i j} \mid x_{i j}, b_{j}\right) f\left(w_{i j} \mid x_{i j}, b_{j}\right) f\left(x_{i j}\right) d x_{i j} d b_{j}
\end{aligned}
$$


onde a última igualdade e se deve à não-diferenciabilidade (Bolfarine e ArellanoValle, 1998). Assim, após algumas manipulações algébricas, obtemos

$$
\left(\begin{array}{c}
y_{i} \\
w_{i}
\end{array}\right) \stackrel{i i d}{\sim} N_{2}\left[\left(\begin{array}{c}
\alpha+\beta \mu_{x} \\
\mu_{x}
\end{array}\right) ;\left(\begin{array}{cc}
\beta^{2} \sigma_{x}^{2}+\sigma_{\epsilon}^{2} & \beta \sigma_{x}^{2} \\
\beta \sigma_{x}^{2} & \sigma_{x}^{2}+\sigma_{\xi}^{2}
\end{array}\right)\right] .
$$

Para as observações censuradas, temos

$$
\begin{aligned}
P\left(Y_{i j} \geq y_{i j}, w_{i j}\right) & =\int_{y_{i j}}^{+\infty} f\left(z_{i j}, w_{i j}\right) d z_{i j} \\
& =\int_{-\infty}^{+\infty} \int_{-\infty}^{+\infty}\left[\int_{y_{i j}}^{+\infty} f\left(z_{i j}, w_{i j}, x_{i j}, b_{j}\right) d z_{i j}\right] d x_{i j} d b_{j} \\
& =\int_{-\infty}^{+\infty} \int_{-\infty}^{+\infty} \int_{y_{i j}}^{+\infty} f\left(z_{i j} \mid w_{i j}, x_{i j}, b_{j}\right) f\left(w_{i j} \mid x_{i j}, b_{j}\right) f\left(x_{i j}\right) f\left(b_{j}\right) d z_{i} d x_{i j} d b_{j} \\
& =\int_{-\infty}^{+\infty} \int_{-\infty}^{+\infty} \int_{y_{i j}}^{+\infty} f\left(z_{i j} \mid x_{i j}, b_{j}\right) f\left(w_{i j} \mid x_{i j}, b_{j}\right) f\left(x_{i j}\right), f\left(b_{j}\right) d z_{i j} d x_{i j} d b_{j} \\
& =\int_{y_{i j}}^{+\infty} \int_{-\infty}^{+\infty} \int_{-\infty}^{+\infty} f\left(z_{i j} \mid x_{i j}, b_{j}\right) f\left(w_{i j} \mid x_{i j}, b_{j}\right) f\left(x_{i j}\right) f\left(b_{j}\right) d x_{i j} d b_{j} d z_{i j} \\
& =\int_{-\infty}^{+\infty} \int_{y_{i j}}^{+\infty} f\left(z_{i j}, w_{i j}, b_{j}\right) d z_{i j} d b_{j} \\
& =\int_{y_{i j}}^{+\infty} \int_{-\infty}^{+\infty} f\left(z_{i j} \mid w_{i j}, b_{j}\right) f\left(w_{i j}\right) f\left(b_{j}\right) d z_{i j} d b_{j} \\
& =\int_{y_{i j}}^{+\infty} f\left(b_{j}\right) f\left(w_{i j}\right) S\left(y_{i j} \mid w_{i j}, b_{j}\right) d b_{j},
\end{aligned}
$$

$i=1, \ldots, n_{j}$ e $j=1, \ldots, m$, com $S\left(y_{i j} \mid w_{i j}, b_{j}\right)=1-\Phi\left(y_{i j} ; \mu_{c i j}, \sigma_{c}^{2}\right)$, com $\mu_{c i j}=$ $b_{j}+\beta \mu_{x}+\frac{\beta \sigma_{x}^{2}}{\sigma_{x}^{2}+\sigma_{\xi}^{2}}\left(w_{i j}-\mu_{x}\right)$; e $\sigma_{c}^{2}=\beta^{2} \sigma_{x}^{2}\left(\frac{\sigma_{\xi}^{2}}{\sigma_{x}^{2}+\sigma_{\xi}^{2}}\right)+\sigma_{\epsilon}^{2}$ denotam a média e variância condicional de $Y_{i j}$ e $w_{i j}$ dado $b_{j}$. Portanto, a função de verossimilhança pode ser obtida a partir das expressões (6.13) e (6.15) para as observações não censuradas e censuradas, respectivamente. Note que no caso de observações censuradas não temos expressões explícitas para a função de sonbrevivência no modelo log-normal. 


\subsubsection{Estimação dos parâmetros}

Nesta seção discutimos a estimação dos parâmetros pelo método de máxima verossimilhança em que os estimadores são obtidos numericamente. Uma abordagem bayesiana também foi aplicada para a estimação dos parâmetros.

\section{Método de Máxima Verossimilhança}

A função de verossimilhança marginal de $Y_{i j}$ e $w_{i j}, i=1$, ldots, $n_{j}$ e $j=1$, ldots, $m$, é expressa por

$$
\begin{aligned}
L_{j}(\boldsymbol{\theta} ; \mathbf{y}, w, \mathbf{b})= & \int_{-\infty}^{+\infty} \prod_{i=1}^{n_{j}} f\left(y_{i j} \mid b_{j}, w_{i j}\right)^{\delta_{i j}} S\left(y_{i j} \mid b_{j}, w_{i j}\right)^{1-\delta_{i j}} f\left(w_{i j}\right) f\left(b_{j}\right) d b_{j} \\
= & \int_{-\infty}^{+\infty} \prod_{i \in F} f\left(y_{i j} \mid b_{j}, w_{i j}\right) \prod_{j \in C} S\left(y_{i j} \mid w_{i j}, b_{j}\right) \prod_{i=1}^{n_{j}} f\left(w_{i j}\right) f\left(b_{j}\right) d b_{j} \\
= & \int_{-\infty}^{+\infty} \prod_{i \in F} \phi\left(y_{i j}: \mu_{c i j}, \sigma_{c}^{2}\right) \prod_{i \in C}\left(1-\Phi\left(y_{i j}: \mu_{c i j}, \sigma_{c}^{2}\right)\right) \\
& \times \prod_{i=1}^{n_{j}} \phi\left(w_{i j}: \mu_{x}, \sigma_{x}^{2}+\sigma_{\xi}^{2}\right) \phi\left(b_{j}: \alpha ; \sigma_{b}^{2}\right) d b_{j}
\end{aligned}
$$

onde $F$ e $C$ denotam os conjuntos de indivíduos onde $y_{i j}$ é o log do tempo de vida observada e o $\log$ do tempo de censura observada, repsectivamente, $\mu_{c i j}$ e $\sigma_{c}^{2}$, foram defeinidas na Seção 6.3. Aplicando o logaritmo natural em ambos os lados da equação (6.16) tem-se

$$
\begin{aligned}
l(\boldsymbol{\theta})= & \sum_{j=1}^{k}\left\{\log \int_{-\infty}^{+\infty} \prod_{i \in F} \phi\left(y_{i j}: \mu_{c i j} ; \sigma_{c}^{2}\right) \prod_{i \in C}\left(1-\Phi\left(y_{i j}: \mu_{c i j} ; \sigma_{c}^{2}\right)\right)\right. \\
& \left.\times \prod_{i=1}^{n_{j}} \phi\left(w_{i j}: \mu_{x} ; \sigma_{x}^{2}+\sigma_{\xi}^{2}\right) \phi\left(b_{j}: \alpha ; \sigma_{u}^{2}\right) d b_{j}\right\} .
\end{aligned}
$$

Como os estimadores de máxima verossimilhança do vetor de parâmetros $\theta$ não apresentam solução analítica em forma fechada. Assim, será nescessário obter os esti- 
madores de forma similar da Seção 6.2.1.1., isto é, através da maximização numérica. A covariância assintótica dos estimadores de máxima verossimilhança pode ser estimada usando a matriz hessiana que pode ser calculada também numericamente.

\section{Método bayesiano}

Um dos pontos importantes na análise bayesiana consiste em especificar distribuições a priori para todos os parâmetros desconhecidos do modelo. A fim de garantir distribuições a posteriori próprias, adotamos distribuições a priori próprias para todos os parâmetros. Assim, consideramos distribuições normais para os parâmetros $\alpha, \beta$ e $\mu_{x}$, isto é,

$$
\alpha \sim N\left(a_{\alpha}, d_{\alpha}\right),, \beta \sim N\left(a_{\beta}, d_{\beta}\right), \text { e } \mu_{x} \sim N\left(a_{\mu_{x}}, d_{\mu_{x}}\right)
$$

Para o parâmetro de escala, $\sigma_{\epsilon}^{2}$, escolhemos a distribuição gama invertida, $G I\left(\tau_{\epsilon}, T_{\epsilon}\right)$, com densidade

$$
\phi\left(\sigma_{\epsilon}^{2} \mid \tau_{\epsilon}, T_{\epsilon}\right)=\frac{\left(T_{\epsilon}\right)^{\tau_{\epsilon}}}{\Gamma\left(\tau_{\epsilon}\right)}\left(\frac{1}{\sigma_{\epsilon}^{2}}\right)^{\tau_{\epsilon}+1} \exp \left\{-\frac{T_{\epsilon}}{\sigma_{\epsilon}^{2}}\right\} .
$$

Para o parâmetro de escala do efeito aleatório $\sigma_{b}^{2}$, adotamos também a distribuição $G I\left(\tau_{b}, T_{b}\right)$, com densidade

$$
\phi\left(\sigma_{b}^{2} \mid \tau_{b}, T_{b}\right)=\frac{\left(T_{b}\right)^{\tau_{b}}}{\Gamma\left(\tau_{b}\right)}\left(\frac{1}{\sigma_{b}^{2}}\right)^{\tau_{b}+1} \exp \left\{-\frac{T_{b}}{\sigma_{b}^{2}}\right\} .
$$

Finalmente, para o parâmetro de escala do erro de medida $\sigma_{x}^{2}$, consideramos a distribuição $G I\left(\tau_{x}, T_{x}\right)$, com densidade

$$
\phi\left(\sigma_{x}^{2} \mid \tau_{x}, T_{x}\right)=\frac{\left(T_{x}\right)^{\tau_{x}}}{\Gamma\left(\tau_{x}\right)}\left(\frac{1}{\sigma_{x}^{2}}\right)^{\tau_{x}+1} \exp \left\{-\frac{T_{x}}{\sigma_{x}^{2}}\right\}
$$


de (6.9)-(6.12) e de (6.18)-(6.21). Efetuamos todas essas escolhas, temos que a distribuição a posteriori conjunta de todas as quantidades envolvidas é dada por

$$
\begin{aligned}
\pi\left(\alpha, \beta, \mu_{x}, b, \sigma_{\epsilon}^{2}, \sigma_{x}^{2}, \sigma_{b}^{2} \mid Y\right) \propto & \prod_{j=1}^{k}\left[\prod_{i \in F} \phi\left(y_{i j} \mid b_{j}+\beta x_{i j}, \sigma_{\epsilon}^{2}\right) \prod_{i \in C} \phi\left(y_{i j}^{*} \mid b_{j}+\beta x_{i j}, \sigma_{\epsilon}^{2}\right) I_{\left(y_{i j}^{*}>C_{i j}\right)}\right] \\
& \prod_{j=1}^{k} \prod_{i=1}^{n_{j}} \phi\left(w_{i j} \mid x_{i j}, \sigma_{\xi}^{2}\right) \prod_{j=1}^{k} \prod_{i=1}^{n_{j}} \phi\left(x_{i j} \mid \mu_{x}, \sigma_{x}^{2}\right) \prod_{j=1}^{k} \phi\left(b_{j} \mid \alpha, \sigma_{b}^{2}\right) \\
& \exp \left\{-\frac{1}{2 d_{\alpha}}\left(\alpha-a_{\alpha}\right)\right\} \exp \left\{-\frac{1}{2 d_{\beta}}\left(\beta-a_{\beta}\right)\right\} \\
& \exp \left\{-\frac{1}{2 d_{\mu_{x}}}\left(\mu_{x}-a_{\mu_{x}}\right)\right\}\left(\sigma_{\epsilon}^{2}\right)^{\left(\tau_{\epsilon}+1\right)} \exp \left\{-\frac{T_{\epsilon}}{\sigma_{\epsilon}^{2}}\right\} \\
& \left(\sigma_{b}^{2}\right)^{\left(\tau_{b}+1\right)} \exp \left\{-\frac{T_{b}}{\sigma_{b}^{2}}\right\}\left(\sigma_{x}^{2}\right)^{\left(\tau_{x}+1\right)} \exp \left\{-\frac{T_{x}}{\sigma_{x}^{2}}\right\} .
\end{aligned}
$$

onde $y_{i j}^{*}$ denota as observações censuradas. As distribuições condicionais completas, necessárias à implementação do amostrador de Gibbs em ambos os casos, são obtidas de forma direta.

\section{Estudo de Simulação}

O método proposto nos conduz ao seguinte estudo de simulação. Assumindo todas a suposições do modelo apresentado na seção anterior. Usamos o programa R juntamente com o pacote R2WinBUGS (Gelman, 2004) e as seguintes especificações relativas às distribuições a priori:

$$
\begin{aligned}
\alpha & \sim N(0.0,0.01), \\
\beta & \sim N(0.0,0.01), \\
\mu_{x} & \sim N(0.0,0.01), \\
\sigma_{b}^{2} & \sim G I(0.01,0.01), \\
\sigma_{x}^{2} & \sim G I(0.01,0.01) \\
\mathrm{e} & \\
\sigma_{\epsilon}^{2} & \sim G I(0.01,0.01) .
\end{aligned}
$$


Investigamos os resultados com tamanho de amostra $n=50$ para cada grupo $(j=$ 1, 2 e 3). Para cada combinação de parâmetros, realizamos 500 réplicas para o método de máxima verossimilhança, e para o caso bayesiano consideramos um valor de descarte igual a 1000 e uma cadeia de 10000 iterações adicionadas após o descarte com um valor de salto de 10, sendo este último processo importante para reduzir a dependência das cadeias resultando em um tamanho de amostra igual a 1000. A convergência foi diagnosticada usando a visualização do gráfico de traço da estatística de Gelman e Rubin (1992), apresentado com auxílio do pacote CODA. As tabelas que seguem resumem parte dos resultados das simulações, para valores fixo da variância do erro de medida $\left(\sigma_{\xi}^{2}=0,3\right.$ e $\left.\sigma_{\xi}^{2}=0,5\right)$, e permitem comparar valores estimados com diferentes níveis de censura. Nas Tabelas 6.7 e 6.8, verificamos que o método de máxima verossimilhança parece ser mais preciso na estimação dos parâmetros do que o método bayesiano. Com este último método, a estimação da variância do efeito aleatório difere mais do verdadeiro valor do que com o método de máxima verossimilhança, para todos os tamanhos de amostra e níveis de censura. Notamos também que à medida que a proporção do nível de censura aumenta, os valores das estimativas diferem cada vez mais dos valores verdadeiros, em ambos métodos. 
Tabela 6.5: Média das estimativas de Monte Carlo, média da distribuição a posteriori dos parâmetros e os correspondentes erros padrão, com variância do erro de medida $\sigma_{\xi}^{2}=0,3$ e tamanho de amostra $n=50$ para cada grupo $j=1,2 e 3$. Verdadeiros valores: $\alpha=0,5 ; \beta=1,0 ; \mu_{x}=1,0 ; \sigma_{b}^{2}=1,0 ; \sigma_{x}^{2}=1,0$ e $\sigma_{\epsilon}^{2}=1,0$.

\begin{tabular}{cccc}
\hline \hline Censura & Parâmetro & $\begin{array}{c}\text { Máxima } \\
\text { Verossimilhança }\end{array}$ & $\begin{array}{c}\text { Média a } \\
\text { Posteriori }\end{array}$ \\
\hline \multirow{4}{*}{$30 \%$} & & $1,03513(0,59544)$ & $0,78465(0,59582)$ \\
& $\beta$ & $0,99922(0,13211)$ & $1,05278(0,14069)$ \\
& $\mu_{x}$ & $1,00570(0,09246)$ & $0,99956(0,09303)$ \\
& $\sigma_{b}^{2}$ & $0,71879(0,70191)$ & $1,45648(1,12193)$ \\
& $\sigma_{x}^{2}$ & $1,00205(0,15757)$ & $0,96950(0,15231)$ \\
& $\sigma_{\epsilon}^{2}$ & $1,57173(0,26788)$ & $1,20495(0,22240)$ \\
\hline \multirow{4}{*}{$50 \%$} & $\alpha$ & $1,10949(0,61656)$ & $1,06752(0,61060)$ \\
& $\beta$ & $1,00794(0,16206)$ & $1,05848(0,16005)$ \\
& $\mu_{x}$ & $0,99609(0,09022)$ & $1,00164(0,09244)$ \\
& $\sigma_{b}^{2}$ & $0,69303(0,68528)$ & $1,45777(1,12319)$ \\
& $\sigma_{x}^{2}$ & $0,98802(0,15447)$ & $0,97277(0,15204)$ \\
& $\sigma_{\epsilon}^{2}$ & $1,53339(0,28561)$ & $1,40291(0,29499)$ \\
\hline \hline & $\alpha$ & $1,19547(0,72721)$ & $2,07622(0,70962)$ \\
& $\beta$ & $1,00993(0,25099)$ & $1,06941(0,25118)$ \\
& $\mu_{x}$ & $1,00088(0,08812)$ & $0,99918(0,09202)$ \\
& $\sigma_{b}^{2}$ & $0,67673(0,70988)$ & $1,51151(1,21602)$ \\
& $\sigma_{x}^{2}$ & $0,99612(0,15142)$ & $0,97841(0,15449)$ \\
& $\sigma_{\epsilon}^{2}$ & $1,46952(0,49569)$ & $2,13213(0,72990)$ \\
\hline
\end{tabular}


Tabela 6.6: Média das estimativas de Monte Carlo, média da distribuição a posteriori dos parâmetros e os correspondentes erros padrâo com variância do erro de medida $\sigma_{\xi}^{2}=0,5$ e tamanho de amostra $n=50$ para cada grupo $j=1,2 e 3$. Verdadeiros valores: $\alpha=0,5 ; \beta=1,0 ; \mu_{x}=1,0 ; \sigma_{b}^{2}=1,0 ; \sigma_{x}^{2}=1,0$ e $\sigma_{\epsilon}^{2}=1,0$.

\begin{tabular}{cccc}
\hline \hline Censura & Parâmetro & Máxima & Média a \\
& & Verossimilhança & Posteriori \\
\hline \multirow{2}{*}{$30 \%$} & $\alpha$ & $1,30076(0,62259)$ & $1,00295(0,59427)$ \\
& $\beta$ & $1,01082(0,15222)$ & $0,86663(0,12729)$ \\
& $\mu_{x}$ & $1,00263(0,09643)$ & $1,00030(0,10024)$ \\
& $\sigma_{b}^{2}$ & $0,63921(0,63487)$ & $1,46842(1,13332)$ \\
& $\sigma_{x}^{2}$ & $1,00362(0,17832)$ & $1,17459(0,17534)$ \\
$50 \%$ & $\sigma_{\epsilon}^{2}$ & $1,63938(0,30490)$ & $1,41382(0,23246)$ \\
\hline & $\alpha$ & $1,27217(0,61237)$ & $1,30221(0,60539)$ \\
& $\beta$ & $1,01737(0,18147)$ & $0,86829(0,14510)$ \\
& $\mu_{x}$ & $1,00212(0,10385)$ & $0,99895(0,10024)$ \\
& $\sigma_{b}^{2}$ & $0,63963(0,67360)$ & $1,42319(1,11998)$ \\
& $\sigma_{x}^{2}$ & $0,98938(0,17374)$ & $1,17679(0,17587)$ \\
& $\sigma_{\epsilon}^{2}$ & $1,55710(0,35983)$ & $1,64026(0,31450)$ \\
\hline \hline \multirow{4}{*}{$80 \%$} & $\alpha$ & $1,19822(0,68631)$ & $2,35869(0,70321)$ \\
& $\beta$ & $0,99820(0,28017)$ & $0,87489(0,22861)$ \\
& $\mu_{x}$ & $0,99103(0,10291)$ & $0,99575(0,10136)$ \\
& $\sigma_{b}^{2}$ & $0,72109(0,77452)$ & $1,50745(1,24215)$ \\
& $\sigma_{x}^{2}$ & $0,99714(0,17334)$ & $1,17989(0,17455)$ \\
& $\sigma_{\epsilon}^{2}$ & $1,56556(0,60061)$ & $2,45468(0,77873)$ \\
\hline
\end{tabular}




\section{Capítulo 7}

\section{Considerações finais}

\subsection{Conclusões}

Neste trabalho apresentamos modelos com erros de medida em modelos normais-assimétricos e log-normais. No Capítulo 2 apresentamos uma breve introdução dos modelos que são estudados neste trabalho e que as distribuições consideradas são as versões da distribuição normal e log-normal. No Capítulo 3 estudamos a parte inferencial do modelo de Grubbs normal-assimétrico, implementamos a metodologia de influência local com três esquemas de perturbação. Aplicamos estas metodologias a dois conjuntos de dados reais, de Grubbs e Barnett descritos nas Subseções 3.5.1 e 3.5.2, respectivamente. Comparamos o MGSN com o MGN através dos critérios de informação como AIC, BIC e HQ, cujos resultados favorecem a MGSN nestes exemplos, contra a suposição de normalidade. Ainda podemos observar que as conclusões diferem, ao considerarmos o modelo normal-assimétrico contra o modelo normal. Para o conjunto de dados de Barnett seguem os mesmos resultados em relação à 
comparação dos modelos. No Capítulo 4 apresentamos a parte inferencial do modelo com intercepto nulo com erro de medida normal-assimétrico com uma e duas populações, implementamos a metodologia de influência local com três esquemas de perturbação. Ilustramos as técnicas com uma aplicação a um conjunto de dados reais de índice de placa dental (Hadgu e Koch, 1999). Neste caso, também os critérios de informação favorecem o MINSN contra a suposição de normalidade. Notemos que, para o esquema de perturbação de ponderação de casos, os resultados de influência local do MINSN discordam do MINN. No Capítulo 5 apresentamos a parte inferencial do modelo linear misto considerando distribuição normal-assimétrica multivariada para os efeitos aleatórios, sendo este modelo uma extensão univariada de Sahu et al. (2003). Neste capítulo, utilizamos a idéia de influência local Zhu e Lee (2001) em quatro esquemas de perturbação. Aplicamos o conjunto de dados de colesterol de Framingham, neste caso também o modelo favorece o MMLSN contra a suposição de normalidade. No Capítulo 6 apresentamos os modelos de tempo de falha acelerada considerando o modelo log-normal, neste capítulo comparamos os diferentes métodos de estimação como MV, Regressão-calibração, SIMEX, NAIVE, todos os resultados são aproximadamente próximas. Para o modelo de tempo de falha acelerada apresentamos estimação de MV e bayesiana, sendo este último método mais preciso do que a MV.

\subsection{Pesquisas futuras}

No Capítulo 4 foi considerado o modelo de intercepto nulo com erros de medida normal-assimétrico para duas populações. Pretendemos desenvolver a metodologia de influência local neste modelo para vários esquemas de perturbação e fazer aplicações a 
conjunto de dados reais. Para a extensão multivariada de Sahu et al. (2003) podemos explorar com mais detalhes propriedades desta distribuição. Do ponto de vista de aplicações, podemos aplicar em modelos de calibração, particularmente em modelo de Grubbs, descrito no Capítulo 3, nestes modelos podemos derivar a verossimilhança marginal das quantidades observadas de modo que inferência podem ser tratadas usando programas existentes como o algoritmo EM podem ser implementadas. Ainda neste modelo podemos desenvolver a abordagem de influência local e aplicações em conjunto de dados reais em diferentes esquemas de perturbação. Testes de hipóteses podem ser considerados para os modelos estudados neste trabalho. 


\section{Apêndice A}

\section{Lemas Utilizados}

Lema A.1. Seja $\mathrm{Y} \sim N_{n}(\boldsymbol{\mu}, \boldsymbol{\Sigma})$. Então, para todo vetor fixo a de dimensão $k$ e toda matriz fixa $\mathrm{B}$ de dimensão $k \times n$, temos

$$
\begin{array}{r}
E\left[\Phi_{k}(\mathrm{a}+\mathrm{BY} \mid \eta, \Omega)\right]=\Phi_{k}\left(\mathrm{a} \mid \eta-\mathrm{B} \mu, \Omega+\mathrm{B} \Sigma \mathrm{B}^{\top}\right) \\
E\left[\phi_{k}(\mathrm{a}+\mathrm{BY} \mid, \Omega)\right]=\phi_{k}\left(\mathrm{a} \mid \eta-\mathrm{B} \mu, \Omega+\mathrm{B} \Sigma \mathrm{B}^{\top}\right) .
\end{array}
$$

Demonstração. Veja Lachos (2004).

Lema A.2. Seja $\mathrm{Y} \sim N_{p}(\boldsymbol{\mu}, \Sigma)$ e $\mathrm{X} \sim N_{q}(\boldsymbol{\eta}, \boldsymbol{\Omega})$. Então,

$$
\begin{array}{r}
\phi_{p}(\mathrm{y} \mid \mu+\mathrm{Ax}, \Sigma) \phi_{q}(\mathrm{x} \mid \eta, \Omega)=\phi_{p}\left(\mathrm{y} \mid \mu+\mathrm{A} \eta, \Sigma+\mathrm{A} \mathrm{A}^{\top}\right) \\
\times \phi_{q}\left(\mathrm{x} \mid \eta+\Lambda \mathrm{A}^{\top} \Sigma^{-1}(\mathrm{y}-\mu-\mathrm{A} \eta), \Lambda\right)
\end{array}
$$

onde $\Lambda=\left(\Omega^{-1}+\mathbf{A}^{\top} \Sigma^{-1} \mathbf{A}\right)^{-1}$ com matrix fixa $\mathbf{A}$ de dimensão $k \times n$.

Demonstração. Veja Lachos (2004). 
Lema A.3. Seja $X \sim N\left(\eta, \tau^{2}\right)$. Então, para $a \in \mathbb{R}$ constante, temos

$$
\begin{aligned}
E[X \mid X \geq a] & =\eta+\frac{\phi\left(\frac{a-\eta}{\tau}\right)}{1-\Phi\left(\frac{a-\eta}{\tau}\right)} \tau \\
E\left[X^{2} \mid X \geq a\right] & =\eta^{2}+\frac{\phi\left(\frac{a-\eta}{\tau}\right)}{1-\Phi\left(\frac{a-\eta}{\tau}\right)}(\eta+a) \tau
\end{aligned}
$$

Demonstração. Veja Johnson et al. (1994), Seção 10.1.

Lema A.4. Se $U \sim N(0,1)$, então $E\{\Phi(h U+k)\}=\Phi\left\{\frac{k}{\sqrt{1+h^{2}}}\right\}$, para todo real $h$, $k$.

Demonstração. Veja Azzalini (1985). 


\section{Apêndice B}

\section{Demonstração do Teorema 3.1}

Para encontrar a distribuição marginal de $\mathbf{Y}_{i}$ evitamos trabalhar com o índice $i$, correspondente ao $i$-ésimo item, com a finalidade de simplificar a notação. De (3.3)-(3.5) e da definição de distribuição normal-assimétrica multivariada em (1.4), a densidade marginal de $Y$ é obtida calculando a seguinte integral

$$
\begin{aligned}
f_{\mathbf{Y}}(\mathbf{y} \mid \boldsymbol{\theta}) & =\int f(\mathbf{y} \mid x) f(x) d x \\
& =\int 2 \phi_{p}\left(\mathbf{y} \mid \mathbf{a}+1_{p} x, D(\phi)\right) \phi\left(x \mid \mu_{x}, \phi_{x}\right) \Phi\left(\lambda_{x} \frac{x-\mu_{x}}{\phi_{x}^{1 / 2}}\right) d x .
\end{aligned}
$$

A solução da integral é baseada no Lema A.2. Assim, temos o seguinte resultado

$$
\begin{array}{r}
\phi_{p}\left(\mathrm{y} \mid \mathbf{a}+\mathbf{1}_{p} x, D(\phi)\right) \phi\left(\mathrm{X} \mid \mu_{x}, \phi_{x}\right)=\phi_{p}\left(\mathrm{y} \mid \mathbf{a}+\mathbf{1}_{p} \mu_{x}, D(\phi)+\phi_{x} \mathbf{1}_{p} \mathbf{1}_{p}^{\top}\right) \\
\times \phi\left(x \mid \mu_{x}+\Lambda_{x} \mathbf{1}_{p}^{\top} D(\phi)^{-1}\left(\mathbf{y}-\mathbf{a}-1_{p} \mu_{x}\right)\right),
\end{array}
$$

onde $\Lambda_{x}=\left(\phi_{x}^{-1}+1_{p}^{\top} D(\phi)^{-1} 1_{p}\right)^{-1}$.

Demonstração do Teorema 3.1: 
De (B.1) e (B.2) segue que

$$
\begin{aligned}
f_{\mathrm{Y}}(\mathrm{y} \mid \boldsymbol{\theta})= & \int 2 \phi_{p}\left(\mathrm{y} \mid \mathbf{a}+\mathbf{1}_{p} \mu_{x}, D(\phi)+\phi_{x} \mathbf{1}_{p} \mathbf{1}_{p}^{\top}\right) \phi\left(x \mid \mu_{x}+\Lambda_{x} \mathbf{1}_{p}^{\top}, \phi_{x} D(\phi)^{-1}\right. \\
& \left.\times\left(\mathbf{y}-\mathbf{a}-\mathbf{1}_{p} \mu_{x}\right), \Lambda_{x}\right) \Phi\left(\lambda_{x} \frac{x-\mu_{x}}{\phi_{x}^{1 / 2}}\right) d x \\
= & 2 \phi(\mathbf{y} \mid \boldsymbol{\mu}, \boldsymbol{\Sigma}) E\left[\Phi\left(\frac{\lambda_{x}}{\phi_{x}^{1 / 2}}\left(x-\mu_{x}\right)\right)\right]
\end{aligned}
$$

onde $\boldsymbol{\mu}=\mathrm{a}+1_{p} \mu_{x}$ e $\Sigma=D(\phi)+\phi_{x} 1_{p} \mathbf{1}_{p}^{\top}$.

Utilizando (A.1), conclui-se a prova do Teorema, levando a

$$
f_{\mathrm{Y}}(\mathrm{y} \mid \boldsymbol{\theta})=2 \phi_{p}(\mathrm{y} \mid \boldsymbol{\mu}, \boldsymbol{\Sigma}) \Phi\left(\overline{\boldsymbol{\lambda}}_{x}^{\top} \boldsymbol{\Sigma}^{-1 / 2}(\mathrm{y}-\boldsymbol{\mu})\right),
$$

onde $\bar{\lambda}=\frac{\lambda_{x} \phi_{x} \Sigma^{-1 / 2} 1_{p}}{\sqrt{\phi_{x}+\lambda_{x}^{2} \Lambda_{x}}}$. 


\section{Apêndice C}

\section{Cálculo das esperanças do passo E do algoritmo EM}

Neste apêndice apresentamos os cálculos necessários para obter as esperanças do passo E para a implementação do algoritmo EM do modelo de Grubbs normal-assimétrico apresentado no Capítulo 3. Evitamos de trabalhar com o índice $i$, correspondente ao $i$-éssimo item, com a finalidade de simplificar a notação. O passo E para os modelos MINSN e MLMSN são calculados de forma similar.

De (3.3)-(3.5) a densidade conjunta de Y, $x$ e $T$ é obtida por

$$
f_{\mathrm{Y}, x, T}(\mathbf{y}, x, t)=2 \phi_{p}\left(\mathbf{y} \mid \mathbf{a}+\mathbf{1}_{p} x, D(\phi)\right) \phi\left(x \mid \mu_{x}+\phi_{x}^{1 / 2} \delta_{x} t, \phi_{x}\left(1-\delta_{x}^{2}\right)\right) \phi(t \mid 0,1) \mathbf{I}_{(0, \infty)}
$$

Utilizando o Lema A.2 para as funções densidades de y $\mid x$ e $x \mid t$, obtemos o seguinte 
resultado

$$
\begin{aligned}
& \phi_{p}\left(\mathrm{y} \mid \mathbf{a}+\mathbf{1}_{p} x, D(\phi)\right) \phi\left(x \mid \mu_{x}+\phi_{x}^{1 / 2} \delta_{x} t, \phi_{x}\left(1-\delta_{x}^{2}\right)\right)=\phi_{p}\left(\mathrm{y} \mid \mathbf{a}+\mathbf{1}_{p}\left(\mu_{x}+\right.\right. \\
& \left.\left.\phi_{x}^{1 / 2} \delta_{x} t\right), D(\phi)+\phi_{x}\left(1-\delta_{x}^{2}\right) \mathbf{1}_{p} \mathbf{1}_{p}^{\top}\right) \times \phi\left(x \mid \mu_{x}+\phi_{x}^{1 / 2} \delta_{x} t+\Lambda_{x} \mathbf{1}_{p}^{\top} D(\phi)^{-1}\right. \\
& \left.\left(\mathrm{y}-\mathbf{a}-\mathbf{1}_{p}\left(\mu_{x}+\phi_{x}^{1 / 2} \delta_{x} t\right), \Lambda_{x}\right)\right)
\end{aligned}
$$

onde $\Lambda_{x}=\left[\left(\phi_{x}\left(1-\delta_{x}^{2}\right)\right)^{-1}+\mathbf{1}_{p}^{\top} D(\phi)^{-1} \mathbf{1}_{p}\right]^{-1}$.

Note que, a última função densidade de C.1 corresponde à distribuição de $x \mid y \sim$ $N\left(\mu, T_{x}^{2}\right) \mathrm{com}$

$$
\mu=\mu_{x}+\varsigma t+T_{x}^{2} 1_{p}^{\top} D(\phi)^{-1}\left(\mathbf{y}-\mathbf{a}-1_{p}\left(\mu_{x}+\varsigma t\right)\right)
$$

e

$$
T_{x}^{2}=\nu^{2}\left(1+\varsigma^{2} 1_{p}^{\top} D(\phi)^{-1} 1_{p}\right)^{-1}
$$

onde $\varsigma=\phi_{x}^{1 / 2} \delta_{x}, \nu^{2}=\phi_{x}\left(1-\delta_{x}^{2}\right)$ e $T_{x}^{2}=\Lambda_{x}$.

Fazendo

$$
\widehat{x}=E[E[x \mid \boldsymbol{\theta}=\widehat{\boldsymbol{\theta}}, \mathrm{y}]]=E[x \mid \boldsymbol{\theta}=\widehat{\boldsymbol{\theta}}, \mathbf{y}]
$$

temos que,

$$
\widehat{x}=\widehat{r}+\widehat{s} \hat{t}
$$

onde $\widehat{r}=\widehat{\mu_{x}}+\widehat{T_{x}^{2}} 1_{p}^{\top} D(\widehat{\phi})^{-1}\left(\mathbf{y}-\mathbf{a}-\mathbf{1}_{p} \widehat{\mu_{x}}\right)$ e $\widehat{s}=\widehat{\varsigma}\left(1-\widehat{T_{x}^{2}} 1_{p}^{\top} D(\widehat{\phi})^{-1} \mathbf{1}_{p}\right) t$.

A seguir, aplicamos novamente o Lema 2.2 no resultado de C.1, isto é, entre as funções densidades de $\mathrm{Y} \mid t$ e $t$, obtemos o seguinte resultado

$$
\begin{aligned}
& \phi_{p}\left(\mathrm{y} \mid \mathbf{a}+\mathbf{1}_{p}\left(\mu_{x}+\phi_{x}^{1 / 2} \delta_{x} t\right), D(\phi)+\phi_{x}\left(1-\delta_{x}^{2}\right) \mathbf{1}_{p} \mathbf{1}_{p}^{\top}\right) \phi(t \mid 0,1) \mathbf{I}_{(0, \infty)}= \\
& \phi_{p}\left(\mathrm{y} \mid \mathbf{a}+\mathbf{1}_{p} \mu_{x}, D(\phi)+\phi_{x}\left(1-\delta_{x}^{2}\right) \mathbf{1}_{p} \mathbf{1}_{p}^{\top}+\phi_{x} \delta_{x}^{2} \mathbf{1}_{p} \mathbf{1}_{p}^{\top}\right) \times \phi\left(t \mid \Lambda_{T} \phi_{x}^{1 / 2} \delta_{x} \mathbf{1}_{p}^{\top}\right. \\
& \left.\left(D(\phi)+\phi_{x}\left(1-\delta_{x}^{2}\right) \mathbf{1}_{p} \mathbf{1}_{p}^{\top}\right)^{-1}\left(\mathrm{y}-\mathbf{a}-\mu_{x} \mathbf{1}_{p}\right), \Lambda_{T}\right) \mathbf{I}_{(0, \infty)}
\end{aligned}
$$


onde $\Lambda_{T}=\left[1+\phi_{x} \delta_{x}^{2} 1_{p}^{\top}\left(D(\phi)+\phi_{x}\left(1-\delta_{x}^{2}\right)\right)^{-1} 1_{p}\right]^{-1}$

Note que, a última função de densidade de C.3, corresponde à distribuição de $t \mid y$ $N\left(\mu_{T}, M_{T}^{2}\right) \mathbf{I}_{(0, \infty)}$ com

$$
\mu_{T}=\varsigma M_{T}^{2} 1_{p}^{\top}\left(D(\phi)+\nu^{2} 1_{p} 1_{p}^{\top}\right)^{-1}\left(\mathrm{y}-\mathbf{a}+\mu_{x} 1_{p}\right)
$$

e

$$
M_{T}^{2}=\left[1+\varsigma^{2} \mathbf{1}_{p}^{\top}\left(D(\phi)+\nu^{2} \mathbf{1}_{p} \mathbf{1}_{p}^{\top}\right)^{-1} \mathbf{1}_{p}\right]
$$

onde $\varsigma=\phi_{x}^{1 / 2} \delta_{x}, \nu^{2}=\phi_{x}\left(1-\delta_{x}^{2}\right)$ e $M_{T}^{2}=\Lambda_{T}$.

Fazendo

$$
\widehat{t}=E[E[T \mid \boldsymbol{\theta}=\widehat{\boldsymbol{\theta}}, \mathbf{y}]]=E[T \mid \boldsymbol{\theta}=\widehat{\boldsymbol{\theta}}, \mathbf{y}]
$$

e utilizando o Lema A.3, temos que.

$$
\widehat{t}=\widehat{\mu}_{T}+W_{\Phi_{1}}\left(\frac{\widehat{\mu}_{T}}{\widehat{M}_{T}}\right) \widehat{M}_{T}
$$

e

$$
\widehat{t^{2}}=\widehat{\mu}_{T}^{2}+\widehat{M}_{T}^{2}+W_{\Phi_{1}}\left(\frac{\widehat{\mu}_{T}}{\widehat{M}_{T}}\right) \widehat{M}_{T} \widehat{\mu}_{T}
$$

onde $\widehat{\mu}_{T}=\varsigma \widehat{M}_{T}^{2} \mathbf{1}_{p}^{\top}\left(D(\widehat{\phi})+\nu^{2} \mathbf{1}_{p} \mathbf{1}_{p}^{\top}\right)^{-1}\left(\mathbf{y}-\mathbf{a}-\mathbf{1}_{p} \widehat{\mu}_{x}\right)$ e $\widehat{M}_{T}^{2}=\left[1+\widehat{\varsigma}^{2} \mathbf{1}_{p}^{\top}(D(\widehat{\phi})+\right.$ $\left.\left.\widehat{\nu^{2}} 1_{p} 1_{p}^{\top}\right)^{-1} 1_{p}\right]^{-1}$.

Para calcular

$$
\widehat{x^{2}}=E\left[E\left[x^{2} \mid \boldsymbol{\theta}=\widehat{\boldsymbol{\theta}}, \mathbf{y}\right]\right]=E\left[x^{2} \mid \boldsymbol{\theta}=\widehat{\boldsymbol{\theta}}, \mathbf{y}\right]
$$

utilizamos a seguinte definição

$$
E\left[x^{2} \mid \boldsymbol{\theta}=\widehat{\boldsymbol{\theta}}, \mathbf{y}\right]=\operatorname{Var}[x \mid \boldsymbol{\theta}=\widehat{\boldsymbol{\theta}}, \mathbf{y}]+[E[x \mid \boldsymbol{\theta}=\widehat{\boldsymbol{\theta}}, \mathbf{y}]]^{2}
$$

Assim, temos que

$$
\widehat{x^{2}}=\widehat{T}_{x}^{2}+\widehat{r}^{2}+2 \widehat{r} \widehat{s} \widehat{t}+\widehat{s}^{2} \widehat{t}^{2}
$$


onde $\widehat{r}$ e $\widehat{s}$ são definidas como em C.2.

Finalmente, fazendo

$$
\widehat{t x}=E[E[T x \mid \boldsymbol{\theta}=\widehat{\boldsymbol{\theta}}, \mathrm{y}]]=E[T x \mid \boldsymbol{\theta}=\widehat{\boldsymbol{\theta}}, \mathrm{y}],
$$

note que, o resultado é imediato de C.2, isto é,

$$
\widehat{t x}=\widehat{r} \widehat{t}+\widehat{s} \widehat{t^{2}}
$$




\section{Apêndice D}

\section{Cálculo da função escore do MGSN e MINSN}

Neste Apêndice apresentamos os cálculos da função escore do modelo de Grubbs normal-assimétrico (MGSN) e o modelo de intercepto nulo normal-assimétrico de uma população (MINSN) correspondentes às Seções 3.2.1 e 4.2.1, respectivamente.

\section{D.1 Função Escore do MGSN}

Da Seção 3.2.1 vimos que a função escore é obtida derivando-se a função de logverossimilhança em relação ao vetor de parâmetros. Temos que

$$
U(\boldsymbol{\theta})=\frac{\partial \ell(\boldsymbol{\theta})}{\partial \boldsymbol{\theta}}=\sum_{i=1}^{n} U_{i}(\boldsymbol{\theta}),
$$


em que $U_{i}(\boldsymbol{\theta})=\frac{\partial \ell_{i}(\boldsymbol{\theta})}{\partial \boldsymbol{\theta}}=\left(U_{i}(\boldsymbol{\alpha})^{\top}, U_{i}(\phi)^{\top}, U_{i}\left(\mu_{x}\right), U_{i}\left(\phi_{x}\right), U_{i}\left(\lambda_{x}\right)\right)^{\top} \mathrm{e}$

$$
U_{i}(\gamma)=\frac{\partial \ell_{i}(\theta)}{\partial \gamma}=-\frac{1}{2} \frac{\partial \log |\Sigma|}{\partial \gamma}-\frac{1}{2} d_{i \gamma}+\frac{\partial \log K_{i}}{\partial \gamma}
$$

onde $d_{i} \gamma=\frac{\partial d_{i}}{\partial \gamma}, \frac{\partial \log K_{i}}{\partial \gamma}=W_{\Phi_{1}}\left(A_{x} a_{i}\right)\left\{A_{x} \frac{\partial a_{i}}{\partial \gamma}+a_{i} \frac{\partial A_{x}}{\partial \gamma}\right\}, \operatorname{com} W_{\Phi_{1}}(u)=\phi_{1}(u) / \Phi_{1}(u)$, $u \in \mathbb{R}, \gamma=\mu_{x}, \alpha, \phi_{x}, \phi, \lambda_{x}, i=1, \ldots, n$.

Assim,

$$
\begin{aligned}
& \frac{\partial \log |\Sigma|}{\partial \gamma}=0, \quad \gamma=\mu_{x}, \alpha, \lambda_{x} \\
& \frac{\partial \log |\Sigma|}{\partial \phi_{x}}=c^{-1} \frac{c-1}{\phi_{x}}, \quad \frac{\partial \log |\Sigma|}{\partial \phi}=-\frac{\phi_{x}}{c} D^{-2}(\phi) \mathbf{1}_{p}+D^{-1}(\phi) \mathbf{1}_{p}, \\
& d_{i \lambda_{x}}=0, \\
& d_{i \mu_{x}}=-21_{p}^{\top} \Sigma^{-1} X_{i}, \quad d_{i \alpha}=-2 \mathbb{I}_{(p)} \Sigma^{-1} X_{i}, \\
& d_{i \phi_{x}}=-c^{-2} a_{i}^{2} \\
& d_{i \phi}=-D^{-2}(\phi) D\left(\boldsymbol{X}_{i}\right) \boldsymbol{X}_{i}+2 c^{-1} \phi_{x} a_{i} D^{-2}(\phi) \boldsymbol{X}_{i}-c^{-2} \phi_{x}^{2} a_{i}^{2} D^{-2}(\phi) \mathbf{1}_{\boldsymbol{p}}, \\
& \frac{\partial a_{i}}{\partial \gamma}=0, \gamma=\phi_{x}, \lambda_{x} \\
& \frac{\partial a_{i}}{\partial \mu_{x}}=-\mathbf{1}_{\boldsymbol{p}}^{\top} D^{-1}(\phi) \mathbf{1}_{\boldsymbol{p}}, \quad \frac{\partial a_{i}}{\partial \boldsymbol{\alpha}}=-D^{-1}(\psi) \mathbf{1}_{\boldsymbol{p}-\mathbf{1}}, \\
& \frac{\partial a_{i}}{\partial \phi}=-D^{-2}(\phi) \boldsymbol{X}_{i} \\
& \frac{\partial A_{x}}{\partial \gamma}=0, \gamma=\mu_{x}, \alpha \\
& \frac{\partial A_{x}}{\partial \phi}=\frac{\left(2 c+\lambda_{x}^{2}\right)}{2 \lambda_{x}^{2}} A_{x}^{3} D^{-2}(\phi) 1_{\boldsymbol{p}}, \quad \frac{\partial A_{x}}{\partial \phi_{x}}=\frac{\left(2 c+\lambda_{x}^{2}-c^{2}\right)}{2 \phi_{x}^{2} \lambda_{x}^{2}} A_{x}^{3}, \\
& \frac{\partial A_{x}}{\partial \lambda_{x}}=\frac{\phi_{x}}{\Lambda_{x}^{2} \lambda_{x}^{3}} A_{x}^{3}
\end{aligned}
$$

em que $A_{x}=\frac{\lambda_{x} \Lambda_{x}}{\sqrt{\phi_{x}+\lambda_{x}^{2} \Lambda_{x}}}, \Lambda_{x}=\frac{\phi_{x}}{c}, c=1+\phi_{x} 1_{p}^{\top} D^{-1}(\phi) \mathbf{1}_{p}, \quad \boldsymbol{X}_{i}=\left(\mathrm{Y}_{i}-\boldsymbol{\mu}\right)$, $a_{i}=\left(\mathbf{y}_{i}-\boldsymbol{\mu}\right)^{\top} D^{-1}(\phi) \mathbf{1}_{p}, \quad i=1, \ldots, n, \quad \mathbb{I}_{(p)}=\left[0, \mathbf{I}_{p-1}\right]$ de dimensão $(p-1 \times p)$ e $\psi=\left(\phi_{2}, \ldots, \phi_{p}\right)^{\top}$. 


\section{D.2 Função escore do MINSN}

Da Seção 4.2.1 vimos que a função escore é obtida derivando-se a função de logverossimilhança em relação ao vetor de parâmetros. Temos que

$$
U(\boldsymbol{\theta})=\frac{\partial \ell(\boldsymbol{\theta})}{\partial \boldsymbol{\theta}}=\sum_{i=1}^{p} \sum_{j=1}^{n_{i}} U_{i j}(\boldsymbol{\theta}),
$$

em que

$U_{i j}(\theta)=\frac{\partial \ell_{i j}(\theta)}{\partial \theta}=\left(U_{i j}\left(\mu_{x}\right), U_{i j}\left(\beta_{1}\right), \ldots, U_{i j}\left(\beta_{p}\right), U_{i j}\left(\phi_{x}\right), U_{i j}\left(\sigma_{1}^{2}\right), \ldots, U_{i j}\left(\sigma_{p}^{2}\right), U_{i j}\left(\lambda_{x}\right)\right.$, $\left.U_{i j}\left(\sigma_{u}^{2}\right)\right)^{\top}$

$\mathrm{e}$

$$
U_{i j}(\gamma)=\frac{\partial \ell_{i j}(\theta)}{\partial \gamma}=-\frac{1}{2} \frac{\partial \log \left|\Sigma_{i}\right|}{\partial \gamma}-\frac{1}{2} \frac{\partial g_{i j}}{\partial \gamma}+\frac{\partial \log K_{i j}}{\partial \gamma}
$$

em que $\frac{\partial \log K_{i j}}{\partial \gamma}=W_{\Phi_{1}}\left(A_{i} a_{i j}\right)\left[A_{i} \frac{\partial a_{i j}}{\partial \gamma}+a_{i j} \frac{\partial A_{i}}{\partial \gamma}\right], \operatorname{com} W_{\Phi_{1}}(u)=\phi_{1}(u) / \Phi_{1}(u), u \in$ $\mathbb{R}, \gamma=\mu_{x}, \beta_{1}, \ldots, \beta_{p}, \phi_{x}, \lambda_{x}, \sigma_{1}^{2}, \ldots, \sigma_{p}^{2}$ e $\sigma_{u}^{2}, i=1, \ldots, p, j=1, \ldots, n_{i}$.

Assim,

$$
\begin{aligned}
& \frac{\partial \log \left|\Sigma_{i}\right|}{\partial \gamma}=0, \quad \gamma=\mu_{x}, \lambda_{x}, \frac{\partial \log |\Sigma|}{\partial \phi_{x}}=c_{i}^{-1} \frac{c_{i}-1}{\phi_{x}}, \\
& \frac{\partial \log \left|\Sigma_{i}\right|}{\partial \beta_{i}}=2 \frac{c_{i}^{-1} \phi_{x}}{\sigma_{i}^{2}} \beta_{i}, \quad \frac{\partial \log \left|\Sigma_{i}\right|}{\sigma_{u}^{2}}=-\frac{c_{i}^{-1} \phi_{x}}{\sigma_{u}^{4}}+\frac{1}{\sigma_{u}^{2}}, \\
& \frac{\partial \log \left|\Sigma_{i}\right|}{\sigma_{i}^{2}}=-\frac{c_{i}^{-1} \phi_{x}}{\sigma_{i}^{4}} \beta_{i}^{2}+\frac{1}{\sigma_{i}^{2}}, \quad \gamma=\lambda_{x}, \\
& \frac{\partial g_{i j}}{\partial \gamma}=0, \quad \gamma \quad \beta_{0 i}^{\top} \Sigma_{i}^{-1} S_{i j}, \\
& \frac{\partial g_{i j}}{\partial \mu_{x}}=-2 q_{1 i j} \frac{1}{\sigma_{i}^{2}} W_{1 i j}+2 c_{i}^{-1} \phi_{x} \beta_{i}^{2} \frac{1}{\sigma_{i}^{4}} q_{1 i j} W_{1 i j}+2 c_{i}^{-1} \phi_{x} \beta_{i} \frac{1}{\sigma_{u}^{2}} q_{2 i j} \frac{1}{\sigma_{i}^{2}} W_{2 i j} \\
& \frac{\partial g_{i j}}{\partial \beta_{i}}= \\
&=-2 c_{i}^{-1} \phi_{x} \frac{1}{\sigma_{i}^{2}} q_{3 i} \frac{1}{\sigma_{u}^{2}} W_{1 i j} W_{2 i j}, \\
& \frac{\partial g_{i j}}{\partial \phi_{x}}=-c_{i}^{-2} a_{i j}^{2},
\end{aligned}
$$




$$
\begin{array}{ll}
\frac{\partial g_{i j}}{\partial \sigma_{i}^{2}}=-\frac{1}{\sigma_{i}^{4}} W_{1 i j}^{2}+2 c_{i}^{-1} \phi_{x} \frac{1}{\sigma_{i}^{4}} \beta_{i} W_{1 i j}\left[\frac{1}{\sigma_{u}^{2}} W_{2 i j}+\frac{1}{\sigma_{i}^{2}} \beta_{i} W_{1 i j}\right]-c_{i}^{-2} \phi_{x}^{2} a_{i j}^{2} \frac{1}{\sigma_{i}^{4}} \beta_{i}^{2}, \\
\frac{\partial g_{i j}}{\partial \sigma_{u}^{2}}=-\frac{1}{\sigma_{u}^{4}} W_{2 i j}^{2}+2 c_{i}^{-1} \phi_{x} \frac{1}{\sigma_{u}^{4}} W_{2 i j}\left[\frac{1}{\sigma_{u}^{2}} W_{2 i j}+\frac{1}{\sigma_{i}^{2}} \beta_{i} W_{1 i j}\right]-c_{i}^{-2} \phi_{x}^{2} a_{i j}^{2} \frac{1}{\sigma_{u}^{4}}, \\
\frac{\partial a_{i j}}{\partial \gamma}=0, \gamma=\phi_{x} \lambda_{x}, & \frac{\partial a_{i j}}{\partial \beta_{i}}=\frac{1}{\sigma_{i}^{2}}\left[W_{1 i j}-\mu_{x} \beta_{i}\right], \\
\frac{\partial a_{i j}}{\partial \mu_{x}}=-\beta_{0 i}^{\top} D^{-1}\left(\phi_{i}\right) \beta_{0 i}, & \frac{\partial a_{i j}}{\partial \sigma_{u}^{2}}=-\frac{1}{\sigma_{u}^{4}} W_{2 i j}, \\
\frac{\partial a_{i j}}{\partial \sigma_{i}^{2}}=-\beta_{i} \frac{1}{\sigma_{i}^{4}} W_{1 i j}, & \frac{\partial A_{i}}{\phi_{x}}=\frac{\left(2 c_{i}+\lambda_{x}^{2}-c_{i}^{2}\right)}{2 \phi_{x}^{2} \lambda_{x}^{2}} A_{i}^{3}, \\
\frac{\partial A_{i}}{\partial \gamma}=0, \gamma=\mu_{x}, & \frac{\partial A_{i}}{\partial \sigma_{i}^{2}}=\frac{\left(2 c_{i}+\lambda_{x}^{2}\right)}{2 \lambda_{x}^{2}} A_{i}^{3} \frac{1}{\sigma_{i}^{4}} \beta_{i}^{2}, \\
\frac{\partial A_{i}}{\partial \beta_{i}}=-\frac{\left(2 c_{i}+\lambda_{x}^{2}\right)}{\lambda_{x}^{2}} A_{i}^{3} \frac{1}{\sigma_{i}^{2}} \beta_{i}, & \frac{\phi_{x}}{\lambda_{x}^{3} \Lambda_{i}^{2}} A_{i}^{3}, \\
\frac{\partial A_{i}}{\partial \lambda_{x}}=\frac{\left(2 c_{i}+\lambda_{x}^{2}\right)}{2 \lambda_{x}^{2}} A_{i}^{3} \frac{1}{\sigma_{u}^{4}}, & \frac{\partial A_{i}}{\partial \sigma_{u}^{2}}=
\end{array}
$$

em que $A_{i}=\frac{\lambda_{x} \Lambda_{i}}{\sqrt{\phi_{x}+\lambda_{x}^{2} \Lambda_{i}}}, \Lambda_{i}=\frac{\phi_{x}}{c_{i}}, c_{i}=1+\phi_{x} \boldsymbol{\beta}_{0 i}^{\top} D^{-1}\left(\phi_{i}\right) \boldsymbol{\beta}_{0 i}, \quad \boldsymbol{S}_{i j}=\left(\mathbf{z}_{i j}-\boldsymbol{\mu}_{i}\right)$, $W_{1 i j}=y_{i j}-\beta_{i} \mu_{x}, \quad W_{2 i j}=X_{i j}-\mu_{x}, \quad q_{1 i j}=\mu_{x}+c_{i}^{-1} \phi_{x} \frac{1}{\sigma_{i}^{2}} W_{1 i j}, \quad q_{2 i j}=\mu_{x}+$ $c_{i}^{-1} \phi_{x} \frac{1}{\sigma_{u}^{2}} W_{2 i j}, \quad q_{3 i}=1-2 c_{i}^{-1} \phi_{x} \frac{1}{\sigma_{i}^{2}} \beta_{i}^{2}, \quad a_{i j}=\left(\mathbf{z}_{i j}-\boldsymbol{\mu}_{i}\right)^{\top} D^{-1} \boldsymbol{\beta}_{0 i}, \quad i=1, \ldots, p$, $j=1, \ldots, n_{i}$. 


\section{Apêndice E}

\section{Obtenção da matriz de informação de Fisher observada}

Neste Apêndice apresentamos os cálculos para obter a matriz de informação de Fisher observada do modelo de Grubbs normal-assimétrico (MGSN) e modelo de intercepto nulo normal-assimétrico de uma população (MINSN) correspondentes às Seções 3.2.2 e 4.2 .2 , respectivamente.

\section{E.1 Matriz de informação de Fisher observada do MGSN}

Da Seção 3.2.2. a matriz de informação de Fisher observada para o vetor de parâmetros $\theta$ e é denotada por

$$
\mathbf{L}=\frac{\partial^{2} \ell(\boldsymbol{\theta})}{\partial \boldsymbol{\theta} \partial \boldsymbol{\theta}^{\top}}=\sum_{i=1}^{n} \frac{\partial^{2} \ell_{i}(\boldsymbol{\theta})}{\partial \boldsymbol{\theta} \partial \boldsymbol{\theta}^{\top}} .
$$


Assim, podemos escrever cada elemento da matriz de informação de Fisher observada como

$$
\mathbf{J}_{i}=-\mathbf{L}_{i}(\boldsymbol{\theta})=-\frac{\partial^{2} \ell_{i}(\boldsymbol{\theta})}{\partial \gamma \partial \tau^{\top}},
$$

em que $\frac{\partial^{2} \ell_{i}}{\partial \gamma \partial \tau^{\top}}=-\frac{1}{2} \frac{\partial^{2} \log |\Sigma|}{\partial \gamma \partial \tau^{\top}}-\frac{1}{2} d_{i \gamma \tau^{\top}}+\frac{\partial^{2} \log K_{i}}{\partial \gamma \partial \tau^{\top}}$, com

$$
\begin{aligned}
\frac{\partial^{2} \log K_{i}}{\partial \gamma \partial \tau^{\top}}= & W_{\Phi_{1}}\left(A_{x} a_{i}\right)\left\{\frac{\partial A_{x}}{\partial \gamma} \frac{\partial a_{i}}{\partial \tau^{\top}}+A_{x} \frac{\partial^{2} a_{i}}{\partial \gamma \partial \tau^{\top}}+\frac{\partial a_{i}}{\partial \gamma} \frac{\partial A_{x}}{\partial \tau^{\top}}+a_{i} \frac{\partial^{2} A_{x}}{\partial \gamma \partial \tau^{\top}}\right\} \\
& +\triangle_{\Phi_{1}}\left(A_{x} a_{i}\right)\left\{A_{x} \frac{\partial a_{i}}{\partial \gamma}+a_{i} \frac{\partial A_{x}}{\partial \gamma}\right\}\left\{A_{x} \frac{\partial a_{i}}{\partial \tau^{\top}}+a_{i} \frac{\partial A_{x}}{\partial \tau^{\top}}\right\}
\end{aligned}
$$

em que $\Delta_{\Phi_{1}}(u)=W_{\Phi_{1}}^{\prime}(u)=-W_{\Phi_{1}}(u)\left(u+W_{\Phi_{1}}(u)\right), u \in \mathbb{R}, d_{i \gamma \tau^{\top}}=\frac{\partial^{2} d_{i}}{\partial \gamma \partial \tau^{\top}}$, $\gamma, \tau=\mu_{x}, \alpha, \phi_{x}, \phi, \lambda_{x}$

Assim,

$$
\begin{aligned}
& \frac{\partial^{2} \log |\Sigma|}{\partial \tau \partial \gamma^{\top}}=0, \quad \tau=\mu_{x}, \boldsymbol{\alpha}, \lambda_{x} ; \quad \gamma=\mu_{x}, \boldsymbol{\alpha}, \phi_{x}, \phi, \lambda_{x} \\
& \frac{\partial^{2} \log |\Sigma|}{\partial \phi_{x} \partial \phi_{x}}=-\frac{1}{c^{2} \phi_{x}^{2}}(c-1)^{2}, \frac{\partial^{2} \log |\Sigma|}{\partial \phi_{x} \partial \phi^{\top}}=-c^{-2} 1_{p}^{\top} D^{-2}(\phi), \\
& \frac{\partial^{2} \log |\Sigma|}{\partial \phi \partial \phi^{\top}}=-D^{-2}(\phi)-c^{-2} \phi_{x}^{2} D^{-1}(\phi) M D^{-1}(\phi)+2 c^{-1} \phi_{x} D^{-3}(\phi) \text {, } \\
& d_{i \mu_{x} \mu_{x}}=21_{p}^{\top} \Sigma^{-1} 1_{p} \\
& d_{i \mu_{x} \boldsymbol{\alpha}^{\top}}=21_{p}^{\top} \boldsymbol{\Sigma}^{-1} \mathbb{I}_{(p)}^{\top} \text {, } \\
& d_{i \mu_{x} \phi_{x}}=2 \frac{(c-1)}{c^{2} \phi_{x}} a_{i} \\
& d_{i \mu_{x}} \phi^{\top}=2 c^{-1} \boldsymbol{X}_{i}^{\top} \Sigma^{-1} D^{-1}(\phi), \\
& d_{i \alpha \alpha^{\top}}=2 \mathbb{I}_{(p)} \Sigma^{-1} \mathbb{I}_{(p)}^{\top}, \\
& d_{i \alpha \phi_{x}}=2 c^{-2} a_{i} D^{-1}(\psi) 1_{p-1}, \\
& d_{i \alpha \phi^{\top}}=2 \mathbb{I}_{(p)} \Sigma^{-1} D^{-1}(\phi)\left\{D\left(X_{i}\right)-c^{-1} \phi_{x} a_{i}\right\}, \\
& d_{i \phi_{x} \phi_{x}}=2 \frac{c^{-3}}{\phi_{x}}(c-1) a_{i}^{2}, \\
& d_{i \phi_{x}} \phi^{\top}=\left(-2 c^{-3} \phi_{x} a_{i}^{2} D^{-2}(\boldsymbol{\phi}) \mathbf{1}_{p}+2 c^{-2} a_{i} D^{-2}(\boldsymbol{\phi}) \boldsymbol{X}_{i}\right)^{\top},
\end{aligned}
$$




$$
\begin{aligned}
& d_{i \phi \phi^{\top}}=2 D^{-3}(\phi) D^{2}\left(\boldsymbol{X}_{i}\right)-4 c^{-1} \phi_{x} a_{i} D^{-3}(\phi) D\left(\boldsymbol{X}_{i}\right) \\
& -2 c^{-1} \phi_{x} D^{-2}(\phi) \boldsymbol{X}_{i} \boldsymbol{X}_{i}^{\top} D^{-2}(\phi) \\
& +2 c^{-2} \phi_{x}^{2} D^{-2}(\phi) \boldsymbol{X}_{i} \boldsymbol{X}_{i}^{\top} M D^{-1}(\phi) \\
& +2 c^{-2} \phi_{x}^{2} a_{i}^{2} D^{-3}(\phi)-2 c^{-3} \phi_{x}^{3} a_{i}^{2} D^{-1}(\phi) M D^{-1}(\phi) \\
& +2 c^{-2} \phi_{x}^{2} D^{-1}(\phi) M \boldsymbol{X}_{i} \boldsymbol{X}_{i}^{\top} D^{-2}(\phi), \\
& \frac{\partial^{2} a_{i}}{\partial \gamma \partial \tau^{\top}}=0, \gamma=\mu_{x}, \alpha, \phi_{x}, \lambda_{x} \quad \tau=\mu_{x}, \alpha, \phi_{x}, \lambda_{x} ; \\
& \frac{\partial^{2} a_{i}}{\partial \mu_{x} \partial \phi^{\top}}=1_{p}^{\top} D^{-2}(\phi) \\
& \frac{\partial^{2} a_{i}}{\partial \alpha \partial \phi^{\top}}=\mathbb{I}_{(p)} D^{-2}(\phi) \\
& \frac{\partial^{2} a_{i}}{\partial \phi \partial \phi^{\top}}=2 D\left(\boldsymbol{X}_{i}\right) D^{-3}(\boldsymbol{\phi}) \\
& \frac{\partial^{2} A_{x}}{\partial \phi_{x} \partial \phi_{x}}=-\frac{\lambda_{x}^{2}+1}{\lambda_{x}^{2} \phi_{x}^{3}} A_{x}^{3}+\frac{3\left(2 c+\lambda_{x}^{2}-c^{2}\right)^{2}}{4 \lambda_{x}^{4} \phi_{x}^{4}} A_{x}^{5}, \\
& \frac{\partial^{2} A_{x}}{\partial \phi_{x} \partial \phi^{\top}}=\left\{\frac{(c-1)}{\lambda_{x}^{2} \phi_{x}} A_{x}^{3}+\frac{3\left(2 c+\lambda_{x}^{2}\right)\left(2 c+\lambda_{x}^{2}-c^{2}\right)}{4 \lambda_{x}^{4} \phi_{x}^{2}} A_{x}^{5}\right\} 1_{p}^{\top} D^{-2}(\phi), \\
& \frac{\partial^{2} A_{x}}{\partial \phi_{x} \partial \lambda_{x}}=\frac{c-2}{\lambda_{x}^{3} \Lambda_{x} \phi_{x}} A_{x}^{3}+\frac{3\left(2 c+\lambda_{x}^{2}-c^{2}\right)}{2 \lambda_{x}^{5} \Lambda_{x}^{2} \phi_{x}} A_{x}^{5}, \\
& \frac{\partial^{2} A_{x}}{\partial \phi \partial \phi^{\top}}=\left\{-\frac{\phi_{x}}{\lambda_{x}^{2}} A_{x}^{3}+\frac{3\left(2 c+\lambda_{x}^{2}\right)^{2}}{4 \lambda_{x}^{4}} A_{x}^{5}\right\} D^{-1}(\phi) M D^{-1}(\phi) \\
& -\frac{2 c+\lambda_{x}^{2}}{\lambda_{x}^{2}} D^{-3}(\phi) A_{x}^{3}, \\
& \frac{\partial^{2} A_{x}}{\partial \phi \partial \lambda_{x}}=\frac{\phi_{x} A_{x}^{3}}{2 \lambda_{x}^{5} \Lambda_{x}^{2}}\left\{3 A_{x}^{2}\left(2 c+\lambda_{x}^{2}\right)-4 \lambda_{x}^{2} \Lambda_{x}\right\} D^{-2}(\phi) \mathbf{1}_{p}, \\
& \frac{\partial^{2} A_{x}}{\partial \lambda_{x} \partial \lambda_{x}}=-\frac{3 \phi_{x}}{\lambda_{x}^{4} \Lambda_{x}^{2}} A_{x}^{3}+\frac{3 \phi_{x}^{2}}{\lambda_{x}^{6} \Lambda_{x}^{4}} A_{x}^{5}
\end{aligned}
$$

em que $A_{x}=\frac{\lambda_{x} \Lambda_{x}}{\sqrt{\phi_{x}+\lambda_{x}^{2} \Lambda_{x}}}, \Lambda_{x}=\frac{\phi_{x}}{c}, \quad c=1+\phi_{x} \mathbf{1}_{p}^{\top} D^{-1}(\phi) \mathbf{1}_{p}, \boldsymbol{X}_{i}=\left(\mathbf{Y}_{i}-\boldsymbol{\mu}\right)$, $M=D^{-1}(\phi) 1_{p} \mathbf{1}_{p}^{\top} D^{-1}(\phi), \psi=\left(\phi_{2}, \ldots, \phi_{p}\right)^{\top}, \mathbb{I}_{(p)}=\left[0, \mathbf{I}_{p-1}\right]$ de dimensão $(p-1 \times p)$ e $a_{i}=\left(\mathrm{y}_{i}-\boldsymbol{\mu}\right)^{\top} D^{-1}(\phi) \mathbf{1}_{p}, i=1, \ldots, n$. 


\section{E.2 Matriz de informação de Fisher observada do MINSN}

Da Seção 4.2.2. a matriz de informação de Fisher observada para o vetor de parâmetros $\theta$ é denotada por

$$
\mathbf{L}=\frac{\partial^{2} \ell(\boldsymbol{\theta})}{\partial \boldsymbol{\theta} \partial \boldsymbol{\theta}^{\top}}=\sum_{i=1}^{p} \sum_{j=1}^{n_{i}} \frac{\partial^{2} \ell_{i j}(\boldsymbol{\theta})}{\partial \boldsymbol{\theta} \partial \boldsymbol{\theta}^{\top}} .
$$

Assim, podemos escrever cada elemento da matriz de informação de Fisher observada como

$$
\mathbf{J}_{i j}=-\mathbf{L}_{i j}\left(\boldsymbol{\theta} \mid \boldsymbol{y}_{i j}\right)=-\left[\frac{\partial^{2} \ell_{i j}(\boldsymbol{\theta})}{\partial \gamma \partial \boldsymbol{\tau}^{\top}}\right],
$$

em que $\frac{\partial^{2} \ell_{i j}}{\partial \gamma \partial \tau^{\top}}=-\frac{1}{2} \frac{\partial^{2} \log \left|\Sigma_{i}\right|}{\partial \gamma \partial \tau^{\top}}-\frac{1}{2} \frac{\partial^{2} g_{i j}}{\gamma \tau^{\top}}+\frac{\partial^{2} \log K_{i j}}{\partial \gamma \partial \tau^{\top}}, \mathrm{com}$

$$
\begin{aligned}
\frac{\partial^{2} \log K_{i j}}{\partial \gamma \partial \tau^{\top}}= & W_{\Phi_{1}}\left(A_{i} a_{i j}\right)\left\{\frac{\partial A_{i}}{\partial \gamma} \frac{\partial a_{i j}}{\partial \tau^{\top}}+A_{i} \frac{\partial^{2} a_{i j}}{\partial \gamma \partial \tau^{\top}}+\frac{\partial a_{i j}}{\partial \gamma} \frac{\partial A_{i}}{\partial \tau^{\top}}+a_{i j} \frac{\partial^{2} A_{i}}{\partial \gamma \partial \tau^{\top}}\right\} \\
& +\Delta_{\Phi_{1}}\left(A_{i} a_{i j}\right)\left\{A_{i} \frac{\partial a_{i j}}{\partial \gamma}+a_{i j} \frac{\partial A_{i}}{\partial \gamma}\right\}\left\{A_{i} \frac{\partial a_{i j}}{\partial \tau^{\top}}+a_{i j} \frac{\partial A_{i}}{\partial \tau^{\top}}\right\},
\end{aligned}
$$

em que $\Delta_{\Phi_{1}}(u)=W_{\Phi_{1}}^{\prime}(u)=-W_{\Phi_{1}}(u)\left(u+W_{\Phi_{1}}(u)\right), u \in \mathbb{R}, \gamma, \tau=\mu_{x}, \beta_{1}, \ldots, \beta_{p}, \phi_{x}$, $\lambda_{x}, \sigma_{1}^{2}, \ldots, \sigma_{p}^{2}$ e $\sigma_{u}^{2}$

Assim,

$$
\begin{aligned}
\frac{\partial^{2} \log \left|\Sigma_{i}\right|}{\partial \tau \partial \gamma^{\top}} & =0, \quad \tau=\mu_{x}, \lambda_{x} ; \gamma=\mu_{x}, \beta_{i}, \phi_{x}, \sigma_{i}^{2}, \sigma_{u}^{2} \\
\frac{\partial^{2} \log \left|\Sigma_{i}\right|}{\partial \beta_{i} \partial \beta_{i}}=2 c_{i}^{-1} \phi_{x}\left\{1-2 c_{i}^{-1} \phi_{x} \frac{1}{\sigma_{i}^{2}} \beta_{i}^{2}\right\} \frac{1}{\sigma_{i}^{2}}, & \frac{\partial^{2} \log \left|\Sigma_{i}\right|}{\partial \beta_{i} \partial \phi_{x}}=2 c_{i}^{-2} \frac{1}{\sigma_{i}^{2}} \beta_{i}, \\
\frac{\partial^{2} \log \left|\Sigma_{i}\right|}{\partial \beta_{i} \partial \sigma_{i}^{2}}=-2 c_{i}^{-1} \phi_{x}\left\{\beta_{i}-c_{i}^{-1} \phi_{x} \frac{1}{\sigma_{i}^{2}} \beta_{i}^{3}\right\} \frac{1}{\sigma_{i}^{4}}, & \frac{\partial^{2} \log \left|\Sigma_{i}\right|}{\partial \beta_{i} \partial \sigma_{u}^{2}}=2 c_{i}^{-2} \phi_{x}^{2} \frac{1}{\sigma_{u}^{4} \sigma_{i}^{2}} \beta_{i}, \\
\frac{\partial^{2} \log \left|\Sigma_{i}\right|}{\partial \phi_{x} \partial \phi_{x}}=-c_{i}^{-2} \frac{\left(c_{i}-1\right)^{2}}{\phi_{x}^{2}}, & \frac{\partial^{2} \log \left|\Sigma_{i}\right|}{\partial \phi_{x} \partial \sigma_{i}^{2}}=-c_{i}^{-2} \beta_{i}^{2} \frac{1}{\sigma_{i}^{4}}, \\
\frac{\partial^{2} \log \left|\Sigma_{i}\right|}{\partial \phi_{x} \partial \sigma_{u}^{2}}=-c_{i}^{-2} \frac{1}{\sigma_{u}^{4}}, & \frac{\partial^{2} \log \left|\Sigma_{i}\right|}{\partial \sigma_{i}^{2} \partial \sigma_{u}^{2}}=-c_{i}^{-2} \phi_{x}^{2} \beta_{i}^{2} \frac{1}{\sigma_{i}^{4} \sigma_{u}^{4}},
\end{aligned}
$$




$$
\begin{aligned}
& \frac{\partial^{2} \log \left|\Sigma_{i}\right|}{\partial \sigma_{i}^{2} \partial \sigma_{i}^{2}}=-\frac{1}{\sigma_{i}^{4}}\left\{1+c_{i}^{-2} \phi_{x}^{2} \beta_{i}^{4} \frac{1}{\sigma_{i}^{4}}-2 c_{i}^{-1} \phi_{x} \beta_{i}^{2} \frac{1}{\sigma_{i}^{2}}\right\}, \\
& \frac{\partial^{2} \log \left|\Sigma_{i}\right|}{\partial \sigma_{u}^{2} \partial \sigma_{u}^{2}}=-\frac{1}{\sigma_{u}^{4}}\left\{1+c_{i}^{-2} \phi_{x}^{2} \frac{1}{\sigma_{u}^{4}}-2 c_{i}^{-1} \phi_{x} \frac{1}{\sigma_{u}^{2}}\right\} \text {, } \\
& \frac{\partial^{2} g_{i j}}{\partial \tau \partial \gamma^{\top}}=0, \quad \tau=\lambda_{x} ; \gamma=\mu_{x}, \beta_{i}, \phi_{x}, \lambda_{x} \sigma_{i}^{2}, \sigma_{u}^{2} \\
& \frac{\partial^{2} g_{i j}}{\partial \mu_{x} \partial \mu_{x}}=2 \boldsymbol{\beta}_{0 i}^{\top} \boldsymbol{\Sigma}^{-1} \boldsymbol{\beta}_{0 i} \\
& \frac{\partial^{2} g_{i j}}{\partial \mu_{x} \partial \beta_{i}}=4 \frac{c_{i}^{-2} \beta_{i} \phi_{x}}{\sigma_{u}^{2} \sigma_{i}^{2}} W_{2 i j}+4 \frac{c_{i}^{-2} \beta_{i}^{2} \phi_{x}}{\sigma_{i}^{4}} W_{1 i j}-2 \frac{c_{i}^{-1}}{\sigma_{i}^{2}} W_{1 i j}+2 \frac{c_{i}^{-1} \mu_{x} \beta_{i}}{\sigma_{i}^{2}}, \\
& \frac{\partial^{2} g_{i j}}{\partial \mu_{x} \partial \phi_{x}}=2 \frac{c_{i}^{-2}\left(c_{i}-1\right)}{\phi_{x}} a_{i j} \\
& \frac{\partial^{2} g_{i j}}{\partial \beta_{i} \partial \sigma_{i}^{2}}=-2 \frac{c_{i}^{-1} \phi_{x} \beta_{i}}{\sigma_{i}^{6}} p_{1 i} W_{1 i j}^{2}+2 \frac{c_{i}^{-2} \phi_{x}^{2} \beta_{i}^{3}}{\sigma_{i}^{8}} p_{1 i} W_{1 i j}^{2}-2 \frac{1}{\sigma_{i}^{4}} p_{4 i} q_{1 i j} W_{1 i j} \\
& +2 \frac{c_{i}^{-1} \phi_{x} \beta_{i}}{\sigma_{u}^{2} \sigma_{i}^{4}} p_{1 i} q_{2 i j} W_{2 i j}-2 \frac{c_{i}^{-1} \phi_{x}}{\sigma_{i}^{4} \sigma_{u}^{2}} p_{1 i} q_{3 i} W_{1 i j} W_{2 i j} \\
& +4 \frac{c_{i}^{-2} \phi_{x}^{2} \beta_{i}^{2}}{\sigma_{i}^{6} \sigma_{u}^{2}} p_{1 i} W_{1 i j} W_{2 i j}+2 \frac{c_{i}^{-2} \phi_{x}^{2} \beta_{i}^{4}}{\sigma_{i}^{8}} q_{1 i j} W_{1 i j}+2 \frac{c_{i}^{-2} \phi_{x}^{3} \beta_{i}^{3}}{\sigma_{i}^{4} \sigma_{u}^{4}} W_{2 i j}^{2}, \\
& \frac{\partial^{2} g_{i j}}{\partial \beta_{i} \partial \sigma_{u}^{2}}=2 \frac{c_{i}^{-2} \phi_{x}^{2} \beta_{i}}{\sigma_{u}^{4} \sigma_{i}^{4}} p_{1 i} W_{1 i j}^{2}+2 \frac{c_{i}^{-1} \phi_{x} \beta_{i}}{\sigma_{i}^{2} \sigma_{u}^{4}} p_{5 i} q_{2 i j} W_{2 i j}+2 \frac{c_{i}^{-2} \phi_{x}^{2}}{\sigma_{i}^{2} \sigma_{u}^{6}} p_{5 i} W_{2 i j}^{2} \\
& -2 \frac{c_{i}^{-1} \phi_{x}}{\sigma_{i}^{2} \sigma_{u}^{4}} p_{5 i} q_{3 i} W_{1 i j} W_{2 i j}+2 \frac{c_{i}^{-2} \phi_{x}^{2} \beta_{i}^{2}}{\sigma_{i}^{4} \sigma_{u}^{4}} q_{1 i j} W_{1 i j}+4 \frac{c_{i}^{-3} \phi_{x}^{3} \beta_{i}^{2}}{\sigma_{i}^{4} \sigma_{u}^{6}} W_{1 i j} W_{2 i j}, \\
& \frac{\partial^{2} g_{i j}}{\partial \phi_{x} \partial \phi_{x}}=2 \frac{c_{i}^{3}}{\phi_{x}}\left(c_{i}-1\right) a_{i j}^{2} \\
& \frac{\partial^{2} g_{i j}}{\partial \phi_{x} \partial \sigma_{i}^{2}}=-2 c_{i}^{-3} \phi_{x} a_{i j}^{2} \beta_{i}^{2} \frac{1}{\sigma_{i}^{4}}+2 c_{i}^{-2} a_{i j} \beta_{i} \frac{1}{\sigma_{i}^{4}} W_{1 i j} \\
& \frac{\partial^{2} g_{i j}}{\partial \phi_{x} \partial \sigma_{u}^{2}}=-2 c_{i}^{-3} \phi_{x} a_{i j}^{2} \frac{1}{\sigma_{u}^{4}}+2 c_{i}^{-2} a_{i j} \frac{1}{\sigma_{u}^{4}} W_{2 i j} \\
& \frac{\partial^{2} g_{i j}}{\partial \sigma_{i}^{2} \partial \sigma_{i}^{2}}=2 \frac{c_{i}^{-1} \phi_{x} \beta_{i}}{\sigma_{i}^{6} \sigma_{u}^{2}} p_{2 i} W_{1 i j} W_{2 i j}+2 \frac{c_{i}^{-1} \phi_{x} \beta_{i}^{2}}{\sigma_{i}^{8}} p_{3 i} W_{1 i j}^{2}-2 \frac{c_{i}^{-2} \phi_{x}^{2} \beta_{i}^{2}}{\sigma_{i}^{6}} p_{1 i} a_{i j}^{2}+2 \frac{1}{\sigma_{i}^{6}} W_{1 i j}^{2} \\
& +2 \frac{\phi_{x}^{2} \beta_{i}^{3}}{c_{i}^{2} \sigma_{i}^{8}} a_{i j} W_{1 i j} \\
& \frac{\partial^{2} g_{i j}}{\partial \sigma_{u}^{2} \partial \sigma_{u}^{2}}=2 \frac{c_{i}^{-1} \phi_{x}}{\sigma_{u}^{8}} p_{7 i} W_{2 i j}^{2}+2 \frac{\phi_{x} \beta_{i}}{c_{i} \sigma_{i}^{2} \sigma_{u}^{6}} p_{6 i} W_{1 i j} W_{2 i j}-2 \frac{c_{i}^{-2} \phi_{x}^{2}}{\sigma_{u}^{6}} p_{5 i} a_{i j}^{2}+2 \frac{1}{\sigma_{u}^{6}} W_{2 i j}^{2} \\
& +2 \frac{c_{i}^{-2} \phi_{x}^{2}}{\sigma_{u}^{8}} a_{i j} W_{2 i j}
\end{aligned}
$$




$$
\begin{aligned}
& \frac{\partial^{2} g_{i j}}{\partial \sigma_{u}^{2} \partial \sigma_{i}^{2}}=2 \frac{c_{i}^{-1} \phi_{x} \beta_{i}}{\sigma_{i}^{4} \sigma_{u}^{4}} p_{1 i} W_{1 i j} W_{2 i j}+2 \frac{c_{i}^{-2} \phi_{x}^{2} \beta_{i}^{2}}{\sigma_{i}^{4} \sigma_{u}^{6}} W_{2 i j}^{2}-2 \frac{c_{i}^{-3} \phi_{x}^{3} \beta_{i}^{2}}{\sigma_{i}^{2} \sigma_{u}^{4}} a_{i j}^{2} \\
& +2 \frac{c_{i}^{-2} \phi_{x}^{2} \beta_{i}}{\sigma_{i}^{4} \sigma_{u}^{4}} a_{i j} W_{1 i j}, \\
& \frac{\partial^{2} a_{i j}}{\partial \gamma \partial \tau^{\top}}=0, \quad \gamma=\phi, \quad \tau=\phi_{x}, \lambda_{x}, \\
& \frac{\partial^{2} a_{i j}}{\partial \mu_{x} \partial \mu_{x}}=0 \\
& \frac{\partial^{2} a_{i j}}{\partial \mu_{x} \partial \beta_{i}}=-2 \beta_{i} \frac{1}{\sigma_{i}^{2}} \\
& \frac{\partial^{2} a_{i j}}{\partial \mu_{x} \partial \sigma_{i}^{2}}=\beta_{i}^{2} \frac{1}{\sigma_{i}^{4}}, \\
& \frac{\partial^{2} a_{i j}}{\partial \mu_{x} \partial \sigma_{u}^{2}}=\frac{1}{\sigma_{u}^{4}} \\
& \frac{\partial^{2} a_{i j}}{\partial \beta_{i} \partial \beta_{i}}=-2 \mu_{x} \frac{1}{\sigma_{i}^{2}} \\
& \frac{\partial^{2} a_{i j}}{\partial \beta_{i} \partial \sigma_{i}^{2}}=-\frac{1}{\sigma_{i}^{4}} W_{3 i j} \\
& \frac{\partial^{2} a_{i j}}{\partial \beta_{i} \partial \sigma_{u}^{2}}=0 \text {, } \\
& \frac{\partial^{2} a_{i j}}{\partial \sigma_{i}^{2} \partial \sigma_{i}^{2}}=2 \beta_{i} \frac{1}{\sigma_{i}^{6}} W_{1 i j} \\
& \frac{\partial^{2} a_{i j}}{\partial \sigma_{u}^{2} \partial \sigma_{u}^{2}}=2 \frac{1}{\sigma_{u}^{6}} W_{2 i j}, \\
& \frac{\partial^{2} A_{i}}{\partial \tau \partial \gamma^{\top}}=0, \quad \tau=\mu_{x} ; \quad \gamma=\mu_{x}, \beta_{i}, \phi_{x}, \lambda_{x} \sigma_{i}^{2}, \sigma_{u}^{2} \\
& \frac{\partial^{2} A_{i}}{\partial \beta_{i} \partial \beta_{i}}=-\left\{4 \frac{\phi_{x}}{\lambda_{x}^{2}} A_{i}^{3}-\frac{3\left(2 c_{i}+\lambda_{x}^{2}\right)^{2}}{\lambda_{x}^{4}} A_{i}^{5}\right\} \frac{1}{\sigma_{i}^{4}} \beta_{i}^{2}-\frac{\left(2 c_{i}+\lambda_{x}^{2}\right)}{\lambda_{x}^{2}} A_{i}^{3} \frac{1}{\sigma_{i}^{2}}, \\
& \frac{\partial^{2} A_{i}}{\partial \beta_{i} \partial \phi_{x}}=-\left\{2 \frac{\left(c_{i}-1\right)}{\lambda_{x}^{2} \phi_{x}} A_{i}^{3}+\frac{3\left(2 c_{i}+\lambda_{x}^{2}\right)\left(2 c_{i}+\lambda_{x}^{2}-c_{i}^{2}\right)}{2 \lambda_{x}^{4} \phi_{x}^{2}} A_{i}^{5}\right\} \frac{1}{\sigma_{i}^{2}} \beta_{i}, \\
& \frac{\partial^{2} A_{i}}{\partial \beta_{i} \partial \lambda_{x}}=\frac{\phi_{x} A_{i}^{3}}{\lambda_{x}^{5} \Lambda_{i}^{2}}\left\{-3 A_{i}^{2}\left(2 c_{i}+\lambda_{x}^{2}\right)+4 \lambda_{x}^{2} \Lambda_{i}\right\} \frac{1}{\sigma_{i}^{2}} \beta_{i}, \\
& \frac{\partial^{2} A_{i}}{\partial \beta_{i} \partial \sigma_{i}^{2}}=\left\{2 \frac{\phi_{x}}{\lambda_{x}^{2}} A_{i}^{3}-\frac{3\left(2 c_{i}+\lambda_{x}^{2}\right)^{2}}{2 \lambda_{x}^{4}} A_{i}^{5}\right\} \frac{1}{\sigma_{i}^{6}} \beta_{i}^{3}+\frac{\left(2 c_{i}+\lambda_{x}^{2}\right)}{\lambda_{x}^{2}} A_{i}^{3} \frac{1}{\sigma_{i}^{4}} \beta_{i}, \\
& \frac{\partial^{2} A_{i}}{\partial \beta_{i} \partial \sigma_{u}^{2}}=\left\{2 \frac{\phi_{x}}{\lambda_{x}^{2}} A_{i}^{3}-\frac{3\left(2 c_{i}+\lambda_{x}^{2}\right)^{2}}{2 \lambda_{x}^{4}} A_{i}^{5}\right\} \frac{1}{\sigma_{i}^{2} \sigma_{u}^{4}} \beta_{i}, \\
& \frac{\partial^{2} A_{i}}{\partial \phi_{x} \partial \phi_{x}}=-\frac{\left(\lambda_{x}^{2}+1\right)}{\lambda_{x}^{2} \phi_{x}^{3}} A_{i}^{3}+\frac{3\left(2 c_{i}+\lambda_{x}^{2}-c_{i}^{2}\right)^{2}}{4 \lambda_{x}^{4} \phi_{x}^{4}} A_{i}^{5}, \\
& \frac{\partial^{2} A_{i}}{\partial \phi_{x} \partial \lambda_{x}}=\frac{\left(c_{i}-2\right)}{\lambda_{x}^{3} \Lambda_{i} \phi_{x}} A_{i}^{3}+\frac{3\left(2 c_{i}+\lambda_{x}^{2}-c_{i}^{2}\right)}{2 \lambda_{x}^{5} \Lambda_{i}^{2} \phi_{x}} A_{i}^{5}, \\
& \frac{\partial^{2} A_{i}}{\partial \phi_{x} \partial \sigma_{i}^{2}}=\left\{\frac{\left(c_{i}-1\right)}{\lambda_{x}^{2} \phi_{x}} A_{i}^{3}+\frac{3\left(2 c_{i}+\lambda_{x}^{2}\right)\left(2 c_{i}+\lambda_{x}^{2}-c_{i}^{2}\right)}{4 \lambda_{x}^{4} \phi_{x}^{2}} A_{i}^{5}\right\} \frac{1}{\sigma_{i}^{4}} \beta_{i}^{2}, \\
& \frac{\partial^{2} A_{i}}{\partial \phi_{x} \partial \sigma_{u}^{2}}=\left\{\frac{\left(c_{i}-1\right)}{\lambda_{x}^{2} \phi_{x}} A_{i}^{3}+\frac{3\left(2 c_{i}+\lambda_{x}^{2}\right)\left(2 c_{i}+\lambda_{x}^{2}-c_{i}^{2}\right)}{4 \lambda_{x}^{4} \phi_{x}^{2}} A_{i}^{5}\right\} \frac{1}{\sigma_{u}^{4}},
\end{aligned}
$$




$$
\begin{aligned}
\frac{\partial^{2} A_{i}}{\partial \lambda_{x} \partial \lambda_{x}} & =-\frac{3 \phi_{x}}{\lambda_{x}^{4} \Lambda_{i}^{2}} A_{i}^{3}+\frac{3 \phi_{x}^{2}}{\lambda_{x}^{6} \Lambda_{i}^{4}} A_{i}^{5}, \\
\frac{\partial^{2} A_{i}}{\partial \sigma_{i}^{2} \partial \sigma_{i}^{2}} & =\left\{-\frac{\phi_{x}}{\lambda_{x}^{2}} A_{i}^{3}+\frac{3\left(2 c_{i}+\lambda_{x}^{2}\right)^{2}}{4 \lambda_{x}^{4}} A_{i}^{5}\right\} \frac{1}{\sigma_{i}^{8}} \beta_{i}^{4}-\frac{\left(2 c_{i}+\lambda_{x}^{2}\right)}{\lambda_{x}^{2}} A_{i}^{3} \frac{1}{\sigma_{i}^{6}} \beta_{i}^{2}, \\
\frac{\partial^{2} A_{i}}{\partial \sigma_{i}^{2} \partial \lambda_{x}} & =\frac{\phi_{x} A_{i}^{3}}{2 \lambda_{x}^{5} \Lambda_{i}^{2}}\left\{3 A_{i}^{2}\left(2 c_{i}+\lambda_{x}^{2}\right)-4 \lambda_{x}^{2} \Lambda_{i}\right\} \frac{1}{\sigma_{i}^{4}} \beta_{i}^{2}, \\
\frac{\partial^{2} A_{i}}{\partial \sigma_{u}^{2} \partial \sigma_{u}^{2}} & =\left\{-\frac{\phi_{x}}{\lambda_{x}^{2}} A_{i}^{3}+\frac{3\left(2 c_{i}+\lambda_{x}^{2}\right)^{2}}{4 \lambda_{x}^{4}} A_{i}^{5}\right\} \frac{1}{\sigma_{u}^{8}}-\frac{\left(2 c_{i}+\lambda_{x}^{2}\right)}{\lambda_{x}^{2}} A_{i}^{3} \frac{1}{\sigma_{u}^{6}}, \\
\frac{\partial^{2} A_{i}}{\partial \sigma_{u}^{2} \partial \sigma_{i}^{2}} & =\left\{-\frac{\phi_{x}}{\lambda_{x}^{2}} A_{i}^{3}+\frac{3\left(2 c_{i}+\lambda_{x}^{2}\right)^{2}}{4 \lambda_{x}^{4}} A_{i}^{5}\right\} \frac{1}{\sigma_{i}^{4} \sigma_{u}^{4}} \beta_{i}^{2},
\end{aligned}
$$

em que $A_{i}=\frac{\lambda_{x} \Lambda_{i}}{\sqrt{\phi_{x}+\lambda_{x}^{2} \Lambda_{i}}}, \Lambda_{i}=\frac{\phi_{x}}{c_{i}}, c_{i}=1+\phi_{x} \boldsymbol{\beta}_{0 i}^{\top} D^{-1}\left(\phi_{i}\right) \boldsymbol{\beta}_{0 i}, W_{1 i j}=y_{i j}-\beta_{i} \mu_{x}$ $W_{2 i j}=X_{i j}-\mu_{x}, \quad W_{3 i j}=y_{i j}-2 \beta_{i} \mu_{x}, \quad q_{1 i j}=\mu_{x}+c_{i}^{-1} \phi_{x} \frac{1}{\sigma_{i}^{2}} W_{1 i j}, \quad q_{2 i j}=\mu_{x}+$ $c_{i}^{-1} \phi_{x} \frac{1}{\sigma_{u}^{2}} W_{2 i j}, \quad q_{3 i}=1-2 c_{i}^{-1} \phi_{x} \frac{1}{\sigma_{i}^{2}} \beta_{i}^{2}, \quad a_{i j}=\left(\mathbf{z}_{i j}-\boldsymbol{\mu}_{i}\right)^{\top} D^{-1} \boldsymbol{\beta}_{0 i}, \quad p_{1 i}=\frac{\phi_{x} \beta_{i}^{2}}{c_{i} \sigma_{i}^{2}}-1$, $p_{2 i}=\frac{\phi_{x} \beta_{i}^{2}}{c_{i} \sigma_{i}^{2}}-2, p_{3 i}=\frac{\phi_{x} \beta_{i}^{2}}{c_{i} \sigma_{i}^{2}}-3, p_{4 i}=\frac{2 \phi_{x} \beta_{i}^{2}}{c_{i} \sigma_{i}^{2}}-1, p_{5 i}=\frac{\phi_{x}}{c_{i} \sigma_{u}^{2}}-1, \quad p_{6 i}=\frac{\phi_{x}}{c_{i} \sigma_{u}^{2}}-2$, $p_{7 i}=\frac{\phi_{x}}{c_{i} \sigma_{u}^{2}}-3, i=1, \ldots, p, j=1, \ldots, n_{i}$. 


\section{Apêndice F}

\section{Obtenção da matriz delta}

Neste Apêndice apresentamos os cálculos para obter a matriz $\Delta$ em diferentes esquemas de perturbação do modelo de Grubbs normal-assimétrico (MGSN) e modelo de intercepto nulo normal-assimétrico de uma população (MINSN), definidas nas Seções 3.3. e 4.3, respectivamente.

\section{F.1 Matriz Delta para as Perturbações Aditiva e Multiplicativa do MGSN}

Fornecemos abaixo as derivadas dos elementos $\Delta_{i \theta}^{m}$ da matriz $\Delta$ para os esquemas de perturbação aditivo e multiplicativo do MGSN. Neste caso, a matriz $\Delta$ é dada por

$$
\Delta=\left(\Delta_{1}^{m} \boldsymbol{\theta}, \ldots, \Delta_{n}^{m} \boldsymbol{\theta}\right)
$$


em que

$$
\begin{aligned}
\Delta_{i \boldsymbol{\theta}}^{m}= & \frac{\partial T_{m i}\left(\omega_{i}\right)}{\partial \boldsymbol{\theta}}+W_{\Phi_{1}}\left(A_{x} a_{m i}\left(\omega_{i}\right)\right)\left\{\frac{\partial A_{x}}{\partial \boldsymbol{\theta}} S_{m i}+A_{x} \frac{\partial S_{m i}\left(\omega_{i}\right)}{\partial \boldsymbol{\theta}}\right\} \\
& +A_{x} W_{\Phi_{1}}^{\prime}\left(A_{x} a_{m i}\left(\omega_{i}\right)\right) S_{m i}\left\{A_{x} \frac{\partial a_{m i}\left(\omega_{i}\right)}{\partial \boldsymbol{\theta}}+a_{m i}\left(\omega_{i}\right) \frac{\partial A_{x}}{\partial \boldsymbol{\theta}}\right\}
\end{aligned}
$$

$\operatorname{com} T_{m i}\left(\omega_{i}\right)=-\left(\mathbf{y}_{m i}\left(\omega_{i}\right)-\boldsymbol{\mu}\right)^{\top} \Sigma^{-1} \frac{\partial \mathbf{y}_{m i}\left(\omega_{i}\right)}{\partial \omega_{i}}, S_{m i}\left(\omega_{i}\right)=\mathbf{b}^{\top} D^{-1}(\phi) \frac{\partial \mathbf{y}_{m i}\left(\omega_{i}\right)}{\partial \omega_{i}}, i=$ $1, \ldots, n$.

Então,

$$
\begin{aligned}
& \frac{\partial T_{m i}\left(\omega_{i}\right)}{\partial \boldsymbol{\alpha}}=\mathbf{1}_{p} \Sigma^{-1} \frac{\partial \mathbf{y}_{m i}\left(\omega_{i}\right)}{\partial \omega_{i}} \\
& \frac{\partial T_{m i}\left(\omega_{i}\right)}{\partial \phi_{x}}=c^{-2} a_{m i}\left(\omega_{i}\right) \frac{\partial \mathbf{y}_{m i}\left(\omega_{i}\right)^{\top}}{\partial \omega_{i}} D^{-1}(\phi) \mathbf{1}_{\mathbf{p}} \\
& \frac{\partial T_{m i}\left(\omega_{i}\right)}{\partial \phi^{\top}}=\frac{\partial \mathbf{y}_{m i}\left(\omega_{i}\right)^{\top}}{\partial \omega_{i}} \Sigma^{-1}\left\{D\left(\mathbf{X}_{m i}\left(\omega_{i}\right)\right)-c^{-1} \phi_{x} a_{m i}\left(\omega_{i}\right) \mathbf{I}_{(p)}\right\} \\
& \frac{\partial T_{m i}^{\cdot}\left(\omega_{i}\right)}{\partial \lambda_{x}}=0 . \\
& \frac{\partial S_{m i}\left(\omega_{i}\right)}{\partial \gamma}=0, \quad \gamma=\alpha, \phi, \phi_{x} \\
& \frac{\partial S_{m i}\left(\omega_{i}\right)}{\partial \phi}=-D^{-2}(\phi) \frac{\partial \mathbf{y}_{m i}\left(\omega_{i}\right)}{\partial \omega_{i}}
\end{aligned}
$$

em que $c=1+\phi_{x} \mathbf{1}_{p}^{\top} D^{-1}(\phi) \mathbf{1}_{p}, \quad \boldsymbol{X}_{m i}=\left(\mathbf{y}_{m i}\left(\omega_{i}\right)-\boldsymbol{\mu}\right), \quad \mathbf{I}_{(p)}$ matriz identidade de dimensão $(p \times p)$ e $a_{m i}=\left(\mathbf{y}_{m i}\left(\omega_{i}\right)-\boldsymbol{\mu}\right)^{\top} D^{-1}(\phi) \mathbf{1}_{\boldsymbol{p}}, i=1, \ldots, n$.

\section{F.2 Matriz delta para perturbação do viés multi- plicativo do MGSN}

Fornecemos abaixo os elementos $\Delta_{i}$ da matriz $\Delta$ para o esquema de perturbação do viés multiplicativo do MGSN apresentada na Seção 3.3.3. 
Neste caso, a matriz $\Delta$ é dada por

$$
\Delta=\sum_{i=1}^{n} \frac{\partial^{2} l_{i}(\theta / \omega)}{\partial \theta \partial \omega^{\top}}
$$

em que os elementos da matriz $\frac{\partial^{2} l_{i}(\theta / \omega)}{\partial \theta \partial \omega^{\top}}$ são dados por

$$
\frac{\partial^{2} \ell_{i}}{\partial \gamma \partial \omega^{\top}}=-\frac{1}{2} \frac{\partial^{2} \log |\boldsymbol{\Sigma}|}{\partial \gamma \partial \omega^{\top}}-\frac{1}{2} d_{i} \boldsymbol{\gamma} \boldsymbol{\omega}^{\top}+\frac{\partial^{2} \log K_{i}}{\partial \gamma \partial \boldsymbol{\omega}^{\top}}
$$

com

$$
\begin{aligned}
\frac{\partial^{2} \log K_{i}}{\partial \gamma \partial \omega^{\top}}= & W_{\Phi_{1}}\left(A_{x} a_{i}\right)\left\{\frac{\partial A_{x}}{\partial \gamma} \frac{\partial a_{i}}{\partial \omega^{\top}}+A_{x} \frac{\partial^{2} a_{i}}{\partial \gamma \partial \omega^{\top}}+\frac{\partial a_{i}}{\partial \gamma} \frac{\partial A_{x}}{\partial \omega^{\top}}+a_{i} \frac{\partial^{2} A_{x}}{\partial \gamma \partial \omega^{\top}}\right\} \\
& +\Delta_{\Phi_{1}}\left(A_{x} a_{i}\right)\left\{A_{x} \frac{\partial a_{i}}{\partial \gamma}+a_{i} \frac{\partial A_{x}}{\partial \gamma}\right\}\left\{A_{x} \frac{\partial a_{i}}{\partial \omega^{\top}}+a_{i} \frac{\partial A_{x}}{\partial \omega^{\top}}\right\},
\end{aligned}
$$

$\Delta_{\Phi_{1}}(u)=W_{\Phi_{1}}^{\prime}(u)=-W_{\Phi_{1}}(u)\left(u+W_{\Phi_{1}}(u)\right), u \in \mathbb{R}, d_{i} \gamma \omega^{\top}=\frac{\partial^{2} d_{i}}{\partial \gamma \partial \omega^{\top}}, \gamma=\mu_{x}, \alpha, \phi_{x}, \phi$, $\lambda_{x}, i=1, \ldots, n$.

Assim,

$$
\begin{aligned}
\frac{\partial^{2} \log \left|\Sigma_{\omega}\right|}{\partial \gamma \partial \omega^{\top}} & =0, \quad \gamma=\mu_{x}, \alpha, \\
\frac{\partial^{2} \log \left|\Sigma_{\omega}\right|}{\partial \phi_{x} \partial \omega^{\top}} & =2 c_{\omega}^{-2} \omega^{\top} D^{-1}(\psi), \\
\frac{\partial^{2} \log \left|\Sigma_{\omega}\right|}{\partial \phi \partial \omega^{\top}} & =-2 c_{\omega}^{-1} \phi_{x} D^{-2}(\phi)\left\{D_{1}(\boldsymbol{\omega})-c_{\omega}^{-1} \phi_{x} D^{-1}(\psi) \boldsymbol{b}_{\omega} \boldsymbol{\omega}^{\top} D\left(\boldsymbol{b}_{\omega}\right)\right\}, \\
\frac{\partial d_{i \omega}}{\partial \phi_{x} \partial \boldsymbol{\omega}^{\top}} & =-2 c_{\omega}^{-2} a_{i \omega} \boldsymbol{A}_{i \omega}, \\
\frac{\partial d_{i \omega}}{\partial \phi \partial \boldsymbol{\omega}^{\top}} & =2 D^{-2}(\phi)\left\{q_{i \omega} \mathbb{I}_{(p)} D\left(\boldsymbol{Y}_{i}-\boldsymbol{a}-q_{i \omega} \boldsymbol{b}_{\omega}\right)+c_{\omega}^{-1} \phi_{x} \boldsymbol{A}_{i \omega}^{\top}\left(\boldsymbol{Y}_{i}-\boldsymbol{a}-\boldsymbol{b}_{\omega} q_{i \omega}\right)^{\top} D\left(\boldsymbol{b}_{\boldsymbol{\omega}}\right)\right\}^{\top}, \\
\frac{\partial^{2} a_{i}}{\partial \mu_{x} \partial \boldsymbol{\omega}^{\top}} & =-2 \boldsymbol{\omega}^{\top} D^{-1}(\boldsymbol{\psi}), \\
\frac{\partial^{2} a_{i}}{\partial \boldsymbol{\alpha} \partial \boldsymbol{\omega}^{\top}} & =-D^{-1}(\psi), \\
\frac{\partial^{2} a_{i}}{\partial \phi \partial \boldsymbol{\omega}^{\top}} & =-D^{-2}(\phi) D\left(\boldsymbol{Y}_{i}-\boldsymbol{a}-2 \mu_{x} \boldsymbol{b}_{\omega}\right) \mathbb{I}_{(p)}^{\top},
\end{aligned}
$$




$$
\begin{aligned}
& \frac{\partial^{2} A_{x \omega}}{\partial \phi_{x} \partial \omega^{\top}}=-\left\{\frac{2(c-1)}{\lambda_{x}^{2} \phi_{x}} A_{x \omega}^{3}+\frac{3\left(2 c_{\omega}+\lambda_{x}^{2}\right)\left(2 c_{\omega}+\lambda_{x}^{2}-c_{\omega}^{2}\right)}{2 \lambda_{x}^{4} \phi_{x}^{2}} A_{x \omega}^{5}\right\} \omega^{\top} D^{-1}(\psi), \\
& \frac{\partial^{2} A_{x \omega}}{\partial \phi \partial \omega^{\top}}=\left\{2 \frac{\phi_{x}}{\lambda_{x}^{2}} A_{x \omega}^{3}-\frac{3\left(2 c_{\omega}+\lambda_{x}^{2}\right)^{2}}{2 \lambda_{x}^{4}} A_{x \omega}^{5}\right\} D^{-1}(\psi) \mathbf{b}_{\omega} \omega^{\top} D\left(b_{\omega}\right) D^{-2}(\phi) \\
& +\frac{2 c+\lambda_{x}^{2}}{\lambda_{x}^{2}} A_{x \omega}^{3} D^{-2}(\phi) D\left(\boldsymbol{b}_{\omega}\right) \mathbb{I}_{(p)}^{\top} \\
& \frac{\partial^{2} A_{x \omega}}{\partial \lambda_{x} \partial \omega^{\top}}=\frac{\phi_{x} A_{x \omega}^{3}}{\lambda_{x}^{5} \Lambda_{x \omega}^{2}}\left(-3 A_{x \omega}^{2}\left(2 c_{\omega}+\lambda_{x}^{2}\right)+4 \lambda_{x}^{2} \Lambda_{x \omega}\right) \omega^{\top} D^{-1}(\psi) \\
& \frac{\partial d_{i \omega}}{\partial \mu_{x} \partial \omega^{\top}}=-2 c_{\omega}^{-1} A_{i \omega} \\
& \frac{\partial d_{i \omega}}{\partial \alpha \partial \omega^{\top}}=2 q_{i \omega}\left\{D^{-1}(\psi)-2 c_{\omega}^{-1} \phi_{x} M_{1 \omega}\right\}+2 c_{\omega}^{-1} \phi_{x} D^{-1}(\psi) \omega\left(Y_{i 2}-\alpha\right)^{\top} D^{-1}(\psi) \\
& \text { em que } A_{x \omega}=\frac{\lambda_{x} \Lambda_{x \omega}}{\sqrt{\phi_{x}+\lambda_{x}^{2} \Lambda_{x \omega}}}, \quad \Lambda_{x \omega}=\frac{\phi_{x}}{c_{\omega}}, \quad c_{\omega}=1+\phi_{x} \mathrm{~b}_{\omega}^{\top} D^{-1}(\phi) \mathbf{b}_{\omega}, \quad \psi= \\
& \left(\phi_{2}, \ldots, \phi_{p}\right)^{\top}, \quad a_{i \omega}=\mathrm{X}_{i \omega}^{\top} D^{-1}(\phi) \mathrm{b}_{\omega}, \quad \mathrm{X}_{i \omega}=Y_{i}-\mathbf{a}-\mathrm{b}_{\omega} \mu_{x}, \quad \boldsymbol{A}_{i \omega}=\left(\boldsymbol{Y}_{i 2}-\alpha-\right. \\
& \left.2 q_{i \omega} \omega\right)^{\top} D^{-1}(\psi), \quad Y_{i 2}=\left(Y_{i 2}, \ldots, Y_{i p}\right), \quad M_{1 \omega}=D^{-1}(\psi) \omega \omega^{\top} D^{-1}(\psi) \text { e } q_{i \omega}=\mu_{x}+ \\
& c_{\omega}^{-1} \phi_{x} a_{i \omega}, i=1, \ldots, n \text {. }
\end{aligned}
$$

\section{F.3 Matriz delta para perturbação da variável re- sposta do MINSN}

Fornecemos abaixo os elementos $\Delta_{i j}$ da matriz $\Delta$ para o esquema de perturbação da variável resposta do MINSN apresentada na Seção 5.3.3.

Neste caso, a matriz $\Delta$ é dada por

$$
\Delta=\left(\Delta_{11}(\theta), \ldots, \Delta_{1 n_{1}}(\theta), \ldots, \Delta_{p 1}(\theta), \ldots, \Delta_{p n_{p}}(\theta)\right)
$$

em que

$$
\begin{aligned}
\Delta_{i j}(\boldsymbol{\theta})= & \frac{\partial P_{i j}\left(\omega_{i j}\right)}{\partial \boldsymbol{\theta}}+W_{\Phi_{1}}\left(A_{i} a_{i j}\left(\omega_{i j}\right)\right)\left[\frac{\partial A_{i}}{\partial \boldsymbol{\theta}} Q_{i j}\left(\omega_{i j}\right)+A_{i} \frac{\partial Q_{i j}\left(\omega_{i j}\right)}{\partial \boldsymbol{\theta}}\right] \\
& +A_{i} W_{\Phi_{1}}^{\prime}\left(A_{i} a_{i j}\left(\omega_{j}\right)\right) Q_{i j}\left(\omega_{i j}\right)\left[A_{i} \frac{\partial a_{i j}\left(\omega_{i j}\right)}{\partial \boldsymbol{\theta}}+a_{i j}\left(\omega_{i j}\right) \frac{\partial A_{i}}{\partial \boldsymbol{\theta}}\right]
\end{aligned}
$$


$\operatorname{com} P_{i j}\left(\omega_{i j}\right)=-\left(\mathbf{z}_{i j}\left(\omega_{i j}\right)-\boldsymbol{\mu}_{i}\right)^{\top} \Sigma_{i}^{-1} \frac{\partial \mathbf{z}_{i j}\left(\omega_{i j}\right)}{\partial \omega_{i j}}, Q_{i j}\left(\omega_{i j}\right)=\frac{\partial \mathbf{z}_{i j}\left(\omega_{i j}\right)^{\top}}{\partial \omega_{i j}} D^{-1}\left(\phi_{i}\right) \boldsymbol{\beta}_{0 i}$, $W_{\Phi_{1}}(u)=\phi_{1}(u) / \Phi_{1}(u), W_{\Phi_{1}}^{\prime}(u)=-W_{\Phi_{1}}(u)\left(u+W_{\Phi_{1}}(u)\right), u \in \mathbb{R}, \mathrm{e} \frac{\partial a_{i j}\left(\omega_{i j}\right)}{\partial \theta}$, como no caso perturbado, substituímos $\mathbf{z}_{i j}=\left(X_{i j}, y_{i j}\right)^{\top}$ por $\mathbf{z}_{i j}\left(\omega_{i j}\right)=\left(X_{i j}+S_{i} \omega_{i j}, y_{i j}\right)^{\top}$, $i=1, \ldots, p, j=1, \ldots, n_{i}$. Então

$$
\begin{aligned}
& \frac{\partial P_{i j}\left(\omega_{i j}\right)}{\partial \mu_{x}}=-\frac{\beta_{i}}{\sigma_{i}^{2}}\left(p_{1 i}-\frac{\phi_{x}}{c_{i} \sigma_{u}^{2}}\right) S_{i}, \\
& \frac{\partial P_{i j}\left(\omega_{i j}\right)}{\partial \beta_{i}}=-\mu_{x} \frac{1}{\sigma_{i}^{2}} p_{1 i} S_{i}-\frac{\phi_{x}}{c_{i} \sigma_{u}^{2} \sigma_{i}^{2}} p_{4 i} W_{2 i j} S_{i}-\frac{\phi_{x} \beta_{i}}{c_{i} \sigma_{i}^{4}} p_{1 i} W_{1 i j}\left(\omega_{i j}\right) S_{i}, \\
& \frac{\partial P_{i j}\left(\omega_{i j}\right)}{\partial \phi_{x}}=c_{i}^{-2} \frac{\beta_{i}}{\sigma_{i}^{2}} a_{i j}\left(\omega_{i j}\right) S_{i}, \\
& \frac{\partial P_{i j}\left(\omega_{i j}\right)}{\partial \sigma_{i}^{2}}=-\frac{1}{\sigma_{i}^{4}} p_{4 i} W_{1 i j}\left(\omega_{i j}\right) S_{i}-\frac{\phi_{x} \beta_{i}}{c_{i} \sigma_{u}^{2} \sigma_{i}^{4}} W_{2 i j} S_{i}+\frac{\beta_{i}^{3} \phi_{x}^{2}}{c_{i}^{2} \sigma_{i}^{6}} a_{i j}\left(\omega_{i j}\right) S_{i}, \\
& \frac{\partial P_{i j}\left(\omega_{i j}\right)}{\partial \sigma_{u}^{2}}=-\frac{\phi_{x} \beta_{i}}{c_{i} \sigma_{u}^{4} \sigma_{i}^{2}} W_{2 i j} S_{i}+\frac{\phi_{x}^{2} \beta_{i}}{c_{i}^{2} \sigma_{u}^{4} \sigma_{i}^{2}} a_{i j}\left(\omega_{i j}\right) S_{i}, \\
& \frac{\partial Q_{i j}\left(\omega_{i j}\right)}{\partial \gamma}=0, \gamma=\mu_{x}, \phi_{x}, \lambda_{x}, \sigma_{u}^{2}, \\
& \frac{\partial Q_{i j}\left(\omega_{i j}\right)}{\partial \beta_{i}}=\frac{1}{\sigma_{i}^{2}} S_{i}, \\
& \frac{\partial Q_{i j}\left(\omega_{i j}\right)}{\partial \sigma_{i}^{2}}=-\frac{\beta_{i}}{\sigma_{i}^{4}} S_{i} .
\end{aligned}
$$

em que $c_{i}=1+\phi_{x} \boldsymbol{\beta}_{0 i}^{\top} D^{-1}\left(\phi_{i}\right) \boldsymbol{\beta}_{0 i}, S_{i}$ é o fator de escala, $W_{1 i j}\left(\omega_{i j}\right)=\left(y_{i j}+S_{i} \omega_{i j}-\right.$ $\left.\beta_{i} \mu_{x}\right), \quad W_{2 i j}=X_{i j}-\mu_{x}, \quad p_{1 i}=\frac{\phi_{x} \beta_{i}^{2}}{c_{i} \sigma_{i}^{2}}-1, p_{4 i}=\frac{2 \phi_{x} \beta_{i}^{2}}{c_{i} \sigma_{i}^{2}}-1, i=1, \ldots, p, j=1, \ldots, n_{i}$.

\section{F.4 Matriz delta para perturbação da variável ex- planatória do MINSN}

Fornecemos abaixo os elementos $\Delta_{i j}$ da matriz $\Delta$ para o esquema de perturbação da variável explanatória do MINSN apresentada na Seção 5.3.4. 
Neste caso, a matriz $\Delta$ é dada por

$$
\begin{aligned}
\Delta_{i j}(\boldsymbol{\theta})= & \frac{\partial R_{i j}\left(\omega_{i j}\right)}{\partial \boldsymbol{\theta}}+W_{\Phi_{1}}\left(A_{i} a_{i j}\left(\omega_{i j}\right)\right)\left[\frac{\partial A_{i}}{\partial \boldsymbol{\theta}} V_{i j}\left(\omega_{i j}\right)+A_{i} \frac{\partial V_{i j}\left(\omega_{i j}\right)}{\partial \boldsymbol{\theta}}\right] \\
& +A_{i} W_{\Phi_{1}}^{\prime}\left(A_{i} a_{i j}\left(\omega_{i j}\right)\right) V_{i j}\left(\omega_{i j}\right)\left[A_{i} \frac{\partial a_{i j}\left(\omega_{i j}\right)}{\partial \boldsymbol{\theta}}+a_{i j}\left(\omega_{i j}\right) \frac{\partial A_{i}}{\partial \boldsymbol{\theta}}\right]
\end{aligned}
$$

$\operatorname{com} R_{i j}\left(\omega_{i j}\right)=-\left(\mathbf{z}_{i j}\left(\omega_{i j}\right)-\boldsymbol{\mu}_{i}\right)^{\top} \Sigma_{i}^{-1} \frac{\partial \mathbf{z}_{i j}\left(\omega_{i j}\right)}{\partial \omega_{i j}}, \quad V_{i j}\left(\omega_{i j}\right)=\frac{\partial \mathbf{z}_{i j}\left(\omega_{i j}\right)}{\partial \omega_{i j}} D^{-1}\left(\phi_{i}\right) \boldsymbol{\beta}_{0 i}$, $W_{\Phi_{1}}(u)=\phi_{1}(u) / \Phi_{1}(u), W_{\Phi_{1}}^{\prime}(u)=-W_{\Phi_{1}}(u)\left(u+W_{\Phi_{1}}(u)\right), u \in \mathbb{R}$, e $\frac{\partial a_{i j}\left(\omega_{i j}\right)}{\partial \theta}$ como no caso perturbado, substituímos $\mathbf{z}_{i j}=\left(X_{i j}, y_{i j}\right)^{\top}$ por $\mathbf{z}_{i j}\left(\omega_{i j}\right)=\left(X_{i j}+S_{i} \omega_{i j}, y_{i j}\right)^{\top}$. Então

$$
\begin{aligned}
& \frac{\partial R_{i j}\left(\omega_{i j}\right)}{\partial \mu_{x}}=-\frac{1}{\sigma_{u}^{2}} p_{1 i} S_{i}-\frac{\phi_{x}}{c_{i} \sigma_{u}^{4}} S_{i}, \\
& \frac{\partial R_{i j}\left(\omega_{i j}\right)}{\partial \beta_{i}}=-\mu_{x} \frac{\beta_{i} \phi_{x}}{c_{i} \sigma_{u}^{2} \sigma_{i}^{2}} S_{i}-\frac{\beta_{i} \phi_{x}^{2}}{c_{i}^{2} \sigma_{u}^{4} \sigma_{i}^{2}} W_{2 i j}\left(\omega_{i j}\right) S_{i}-\frac{\phi_{x}}{c_{i} \sigma_{u}^{2} \sigma_{i}^{2}} p_{4 i} W_{1 i j} S_{i}, \\
& \frac{\partial R_{i j}\left(\omega_{j}\right)}{\partial \phi_{x}}=c_{i}^{-2} \frac{1}{\sigma_{u}^{2}} a_{i j}\left(\omega_{i j}\right) S_{i}, \\
& \frac{\partial R_{i j}\left(\omega_{i j}\right)}{\partial \sigma_{i}^{2}}=-\frac{\phi_{x} \beta_{i}}{c_{i} \sigma_{u}^{2} \sigma_{i}^{4}} W_{1 i j} S_{i}+\frac{\phi_{x}^{2} \beta_{i}^{2}}{c_{i}^{2} \sigma_{u}^{2} \sigma_{i}^{6}} a_{i j}^{x}\left(\omega_{i j}\right) S_{i}, \\
& \frac{\partial R_{i j}\left(\omega_{i j}\right)}{\partial \sigma_{u}^{2}}=-\frac{1}{\sigma_{u}^{4}} W_{2 i j}\left(\omega_{i j}\right) S_{i}-2 \frac{\phi_{x}}{c_{i} \sigma_{u}^{6}} W_{2 i j}\left(\omega_{i j}\right) S_{i}-\frac{\phi_{x} \beta_{i}}{c_{i} \sigma_{u}^{4} \sigma_{i}^{2}} W_{1 i j} S_{i}+\frac{\phi_{x}^{2}}{c_{i} \sigma_{u}^{6}} a_{i j}\left(\omega_{i j}\right) S_{i}, \\
& \frac{\partial U_{i j}\left(\omega_{i j}\right)}{\partial \gamma}=0, \quad \gamma=\mu_{x}, \beta_{i}, \phi_{x}, \lambda_{x}, \sigma_{i}^{2} \\
& \frac{\partial U_{i j}\left(\omega_{i j}\right)}{\partial \sigma_{u}^{2}}=-\frac{1}{\sigma_{u}^{4}} S_{i},
\end{aligned}
$$

em que $c_{i}=1+\phi_{x} \boldsymbol{\beta}_{0 i}^{\top} D^{-1}\left(\phi_{i}\right) \boldsymbol{\beta}_{0 i}, S_{i}$ é o fator de escala, $W_{1 i j}=y_{i j}-\beta_{i} \mu_{x}$, $W_{2 i j}\left(\omega_{i j}\right)=\left(X_{i j}+S_{i} \omega_{i j}-\mu_{x}\right), \quad p_{1 i}=\frac{\phi_{x} \beta_{i}^{2}}{c_{i} \sigma_{i}^{2}}-1, \quad p_{4 i}=\frac{2 \phi_{x} \beta_{i}^{2}}{c_{i} \sigma_{i}^{2}}-1, i=1, \ldots, p$, $j=1, \ldots, n_{i}$. 


\section{Apêndice G}

\section{Programas}

\section{G.1 Programa em MATLAB que calcula os EMV do MGSN usando o algoritmo EM}

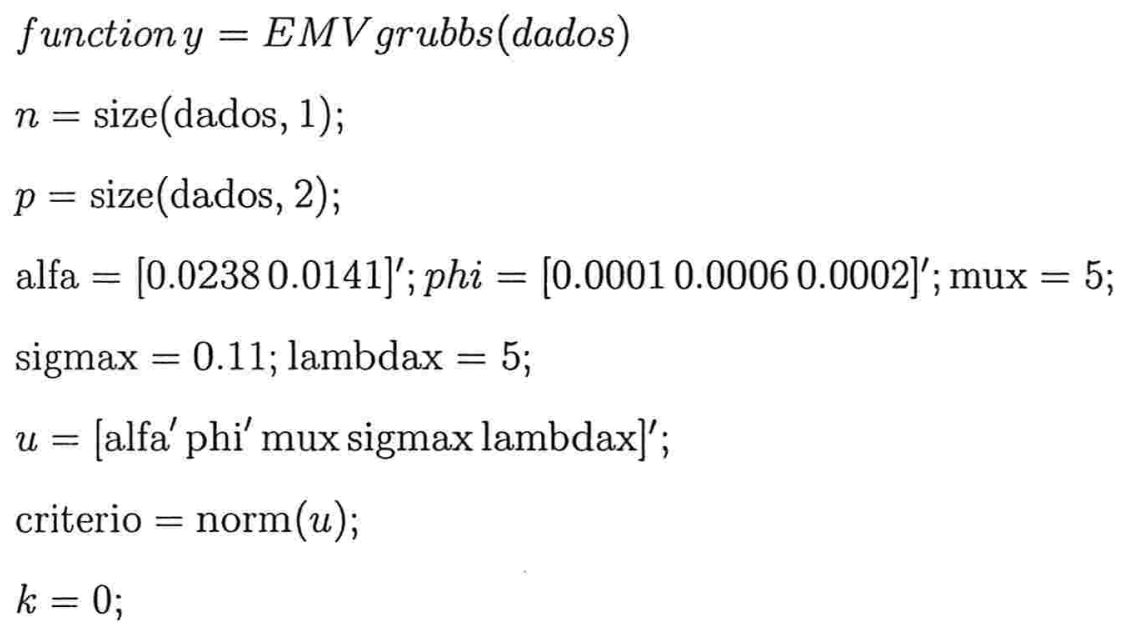




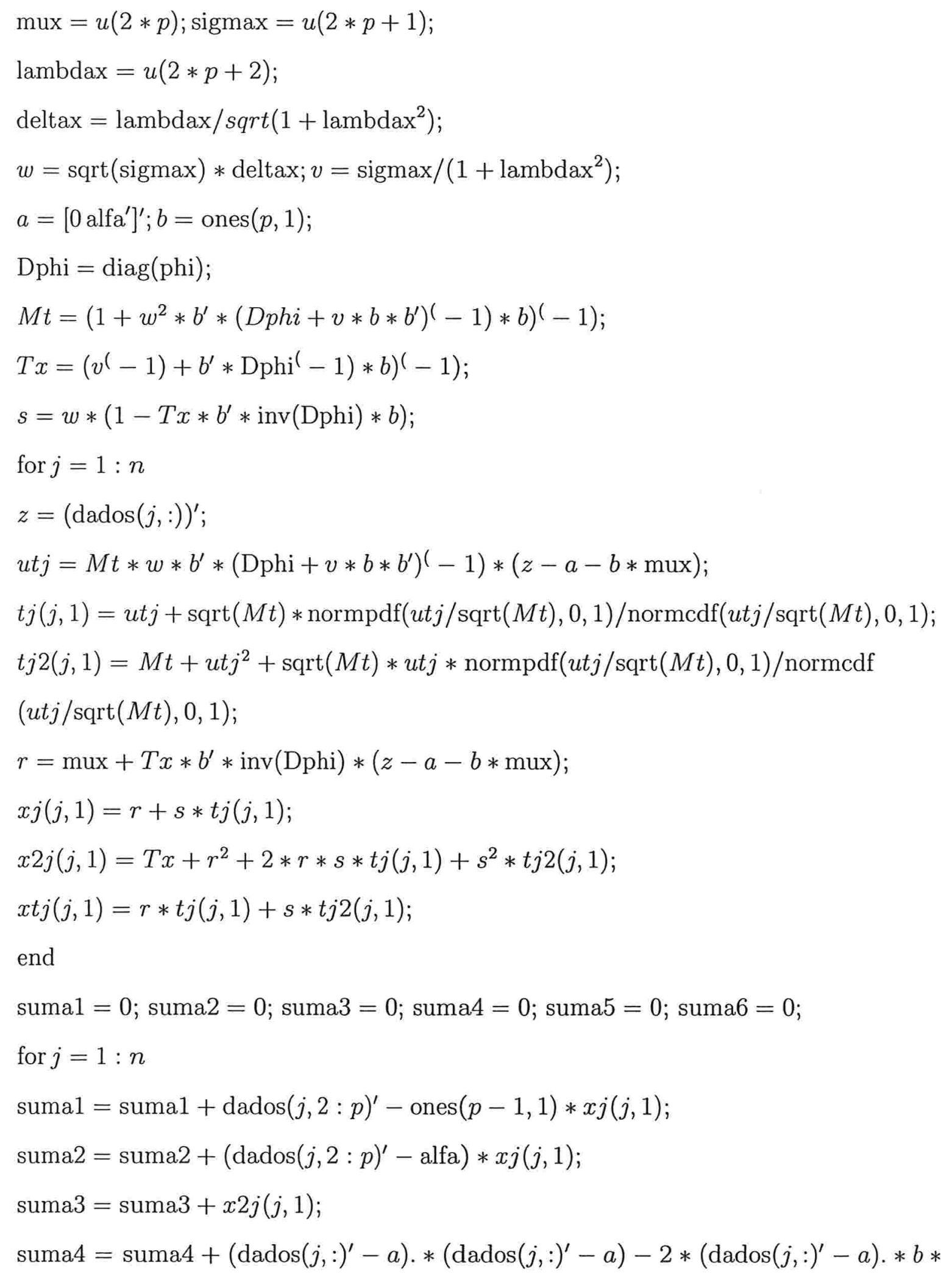




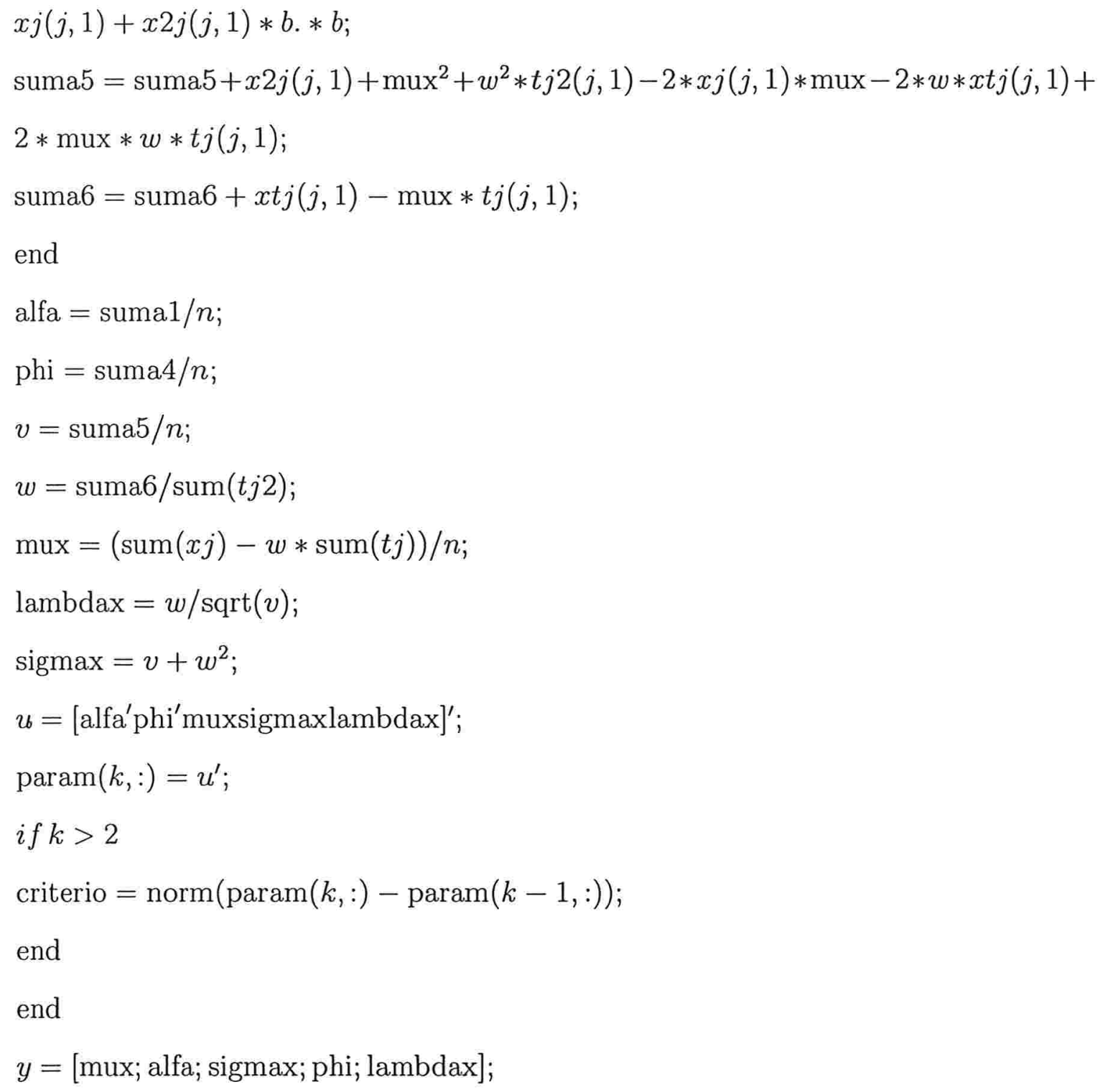




\section{G.2 Programa em MATLAB do gráfico PP tipo}

\section{Healy segundo Azzalini e Capitanio do MGSN}

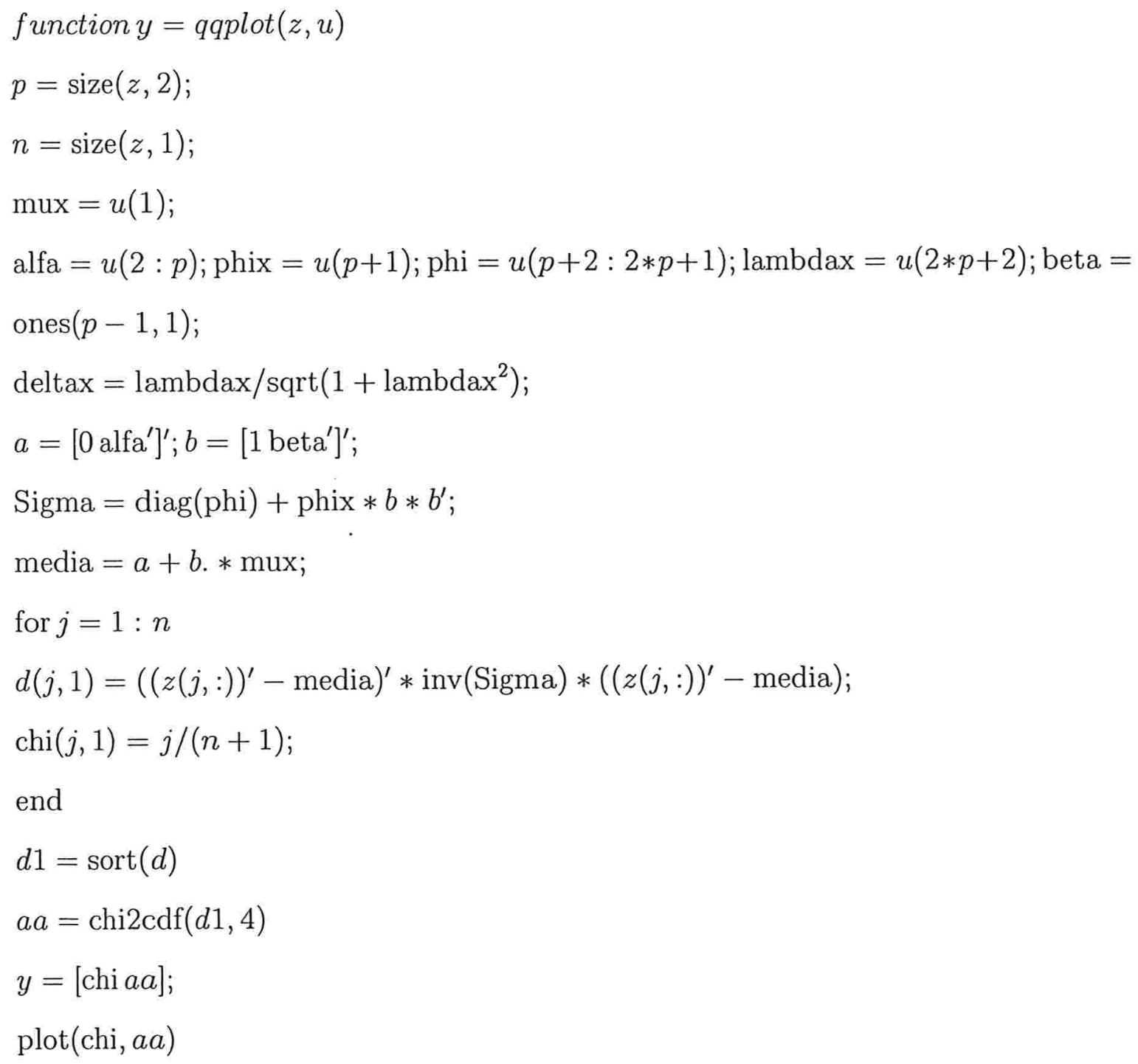




\section{Referências Bibliográficas}

Aigner, D. J., Lovell, C. A. K. e Schmidt, P. (1977). Formulation and estimation of stochastic frontier production function model, Journal of Econometrics 12: 1237.

Aoki, R., Bolfarine, H., Achcar, J. A. e Pinto, J. D. L. (2003). Bayesian analysis of a multivariate null intercept error-in-variables regression model, Journal of Biopharmaceutical Statistics 13: 767-775.

Aoki, R., Bolfarine, H. e Singer, J. M. (2001). Null intercept measurement error regression models, Test 10: 441-457.

Aoki, R., Bolfarine, H. e Singer, J. M. (2002). Null intercept asymptotic efficiency of method of moments estimators under null intercept measurement error regression models, Brazilian Journal of Probability 16: 157-166.

Arellano-Valle, R. B., Bolfarine, H. e Lachos, V. H. (2005). Skew-normal linear mixed models, Journal of Data Science 3: 415-438.

Arellano-Valle, R. B. e Genton, M. G. (2005). Fundamental skew distributions, Journal of Multivariate Analysis 96: 93-116. 
Azzalini, A. (1985). A class of distributions wich includes the normal ones, Scandinavian Journal of Statistics 12: 171-178.

Azzalini, A. (2005). The skew-normal distribution and related multivariate families. http://tango.stat.unipd.it/SN/review.ps.

Azzalini, A. e Capitanio, A. (1999). Statistical applications of the multivariate skewnormal distribution, Journal of the Royal Statistical Society: Series B 61: 579602.

Azzalini, A. e Dalla Valle, A. (1996). The multivariate skew-normal distribution, Biometrika 83: 715-726.

Azzalini, A. e Kotz, S. (2002). Log-skew-normal and log-skew-t distributions as models for family income data. Technical Report, Available at (http://azzalini.stat.unipd.it/SN/income-distr.pdf).

Barnett, V. D. (1969). Simultaneous pairwise linear structural relationships, Biometrics 25: 129-142.

Bayes, C. R. (2005). Modelos normais assimétricos, PhD thesis, IME-USP.

Bedrick, E. J. (2001). An efficient scores test for comparing several measuring devices, Journal of Quality Technology 1: 96-103.

Bolfarine, H. e Arellano-Valle, R. B. (1998). Weak nondifferential measurement error models, Statistics and Probability Letters 40: 279-287.

Box, G. E. P., Jenkins, G. M. e Reinsel, G. C. (1994). Time Series Analysis: Forecasting and Control, San Francisco: Holden Day. 
Brindley, D. A. e Bradley, R. A. (1995). Some new results on grubbs estimators, Journal of the American Statistical Association 80: 711-714.

Broyden, C. G. (1970). The convergence of a class of double-rank minimization algorithms 2, Journal of the Institute of Mathematics and its Applications 6: 222231.

Byrd, R. H., Lu, P., Nocedal, J. e Zhu, C. (1995). A limited memory algorithm for bound constrained optimization, SIAM Journal Scientific Computing 16: 11901208.

Capitanio, A., Azzalini, A. e A., S. (2002). Graphical models for skew-normal variates, Scandinavian Journal of Statistics 29: 652-663.

Carroll, R. J., Ruppert, D. e Stefanski, L. A. (1995). Measurement Error in Nonlinear Models, New York: Chapman and Hall.

Carroll, R. J. e Stefanski, L. A. (1990). Aproximate quasilikelihood estimation in models with surrogate preditors, Journal of the American Statistical Association 85: 652-663.

Chambers, J. (2005). The R Foundation for Statistical Computing - Version 2.2.0, Bell Laboratories (Lucent Technologies).

Chatterjee, S. e Hadi, A. S. (1988). Sensitivity Analysis in Linear Regression, New York: Wiley.

Christensen, R. e Blackwood, L. (1993). Tests for precision and accuracy of multiple measuring devices, Technometrics 35: 411-420.

Clayton, D. G. e Cuzick, J. (1985). Multivariate generalizations of the proportional hazards model, Journal of the Royal Statistical Society A 148: 82-117. 
Cook, D. (1977). Detection of influential observations in linear regression, Technometrics 19: 15-18.

Cook, D. (1986). Assessment of local influence, Journal of the Royal Statistical Society B 48: 133-169.

Cook, J. e Stefanski, L. (1994). A simulation extrapolation method for parametric measurement error models, Journal of the American Statistical Association 89: $1314-1328$.

Cook, R. D. e Weisberg, S. (1982). Residuals and Influence in Regression, London: Chapman and Hall.

Cox, D. e Oakes, D. (1984). Analysis of Survival Data, London: Chapman and Hall.

Cox, D. R. (1972). Regression models and life-tables(with discussion), Journal Royal Statist. Soc. B. 34: 187-220.

Cox, D. R. (1975). Partial likelihood, Biometrika 62: 269-276.

Cuit, H., Kai, M. N. e Zhu, L. (2004). Estimation in mixed effects model with errors in variables, Journal of Multivariate Analysis 91: 53-73.

de Castilho, M. V. (2004). A comparison of statistical techniques for detecting analytical bias in geoanalysis, Geostandars and Geoanalytical Research 28: 277-290.

Dempster, A. P., Laird, N. M. e Rubin, D. B. (1977). Maximum likelihood from incomplete data via the EM algorithm, Journal of the Royal Statistical Society, Series B 39: 1-38.

Diggle, P. J., Liang, K. Y. e Zeger, S. L. (1994). Analysis of Longitudinal Data, Clarendom Press: Oxford. 
Escobar, E. e Meeker, W. (1992). Assessing influence in regression analysis with censured data, Biometrics 48: 507-528.

Fletcher, R. (1970). A new approach to variable metric methods, Computer Journal 13: 317-322.

Fuller, W. A. (1987). Measurement Error Models, New York: John Wiley.

Gabrielsen, A. (1978). Consistency and identificability, Journal of Econometrics 8: 261-263.

Galea-Rojas, M. (1995). Calibração Estrutural e Funcional, PhD thesis, IME-USP.

Galea-Rojas, M., de Castilho, M. V., Bolfarine, H. e de Castro, M. (2003). Detection of analytical bias, Analyst 128: 1073-1081.

Gelman, A. (2004). Running WingBUGS from $R$, Available for download at (http:carn.us.r-project.org/src/contrib/Descriptions/R2WinBUGS.html).

Genton, M. G. (2004). Skew-Elliptical Distribuitions and their Applications: A Journey Beyond Normality, New York: Chapman and Hall.

Genton, M. G., He, L. e Liu, X. (2001). Moments of skew-normal random vectors and their quadratic forms, Statistics and Probability Letters 51: 319-325.

Gimenez, P. (1997). Inferência em modelos com erro nas variáveis através do método do escore corrigido, PhD thesis, IME-USP.

Gimenez, P., Bolfarine, H. e Colosimo, E. A. (1999). Estimation in Weibull regression model with measurement error, Communications in Statistics : Theory and Methods 28: 495-510. 
Goldfarb, D. (1970). A family of variable metric methods derived by variational means, Mathematics of Computation 24: 23-26.

Grubbs, F. E. (1948). On estimating precision of measuring instruments and product variability, Journal of the American Statistical Association 43: 243-264.

Grubbs, F. E. (1973). Errors of measurement, precision, accuracy and the statistical comparision of measuring instruments, Technometrics 15: 53-66.

Hadgu, A. e Koch, G. (1999). Application of generalized estimating equations to a dental randomized clinical trial, Journal of Biopharmaceutical Statistics 9: 161178.

Henderson, C. R. (1984). Aplications of Linear Models in Animal Breeding, University of Guelph: Guelph.

Henze, N. (1986). A probabilistic representation of the skew-normal distribution, Scandinavian Journal of Statistics 13: 271-275.

Hougaard, P. (1987). Survival models for heterogeneous populations derived from stable distributions, Biometrika 73: 387-396.

Hougaard, P. (2000). Analysis of Multivariate Survival Data, New York: SpringerVerlag.

Hu, P., Tsiatis, A. A. e Davidian (1998). Estimating the parameters in the cox model when covariate variables are measured with error, Biometrics 54: 1407-1419.

Hughes, M. D. (1993). Regression dilution in the proportional hazards models, Biometrics 49: 1056-1066. 
Jaech, J. L. (1985). Statistical Analysis of Measurement Errors, Exon Monographs, New York: John Wiley.

Johnson, N., Kotz, S. e Balakrishnan, N. (1994). Continuous Univariate Distributions, Vol. 1, New York: John Wiley.

Keinding, N. G., Andersen, P. K. e Klein, J. P. (1997). The role of frailty models and acclerated failure time models in describing heterogeneity due to omitted covariates., Statistics in Medicine 16: 215-224.

Klein, J. P. (1992). Semiparametric estimation of random effects using the Cox model based on the EM algorithm, Biometrics 48: 795-806.

Klein, J. P. e Moeschberger, M. L. (1997). Survival Analysis: Techniques for Censored and Truncated Data, New York: Springer-Verlag.

Klein, J. P., Pelz, C. e Zhang, M. (1999). Modelling random effects for censored data by a multivariate normal regression model, Biometrics 55: 497-506.

Lachos, V. (2004). Modelos Lineares Mistos Assimétricos, PhD thesis, IME-USP.

Lachos, V. H., Vilca, F. e Galea, M. (2006). Influence diagnostics for the Grubb's model, To appear in Statistcial Papers .

Laird, N. M. e Ware, J. (1982). Random-effects models for longitudinal data, Biometrics 55: 497-506.

Lawless, J. (1982). Statistical Models and Methods for Lifetime Data, New York: John Wiley.

Leurgans, S. (1980). Evaluating Laboratory Measurement Techniques. In Biostatistics Casebol, New York: John Wiley. 
Littell, R. C., Milliken, G. A., Stroup, W. W. e Wolfinger, R. D. (1996). SAS System for Mixed Models., North Carolina: SAS Institute.

Morgan, T. M. e Elashoff, R. M. (1987). Effect of covariate measurement error in randomized clinical trials, Statistics in Medicine 6: 31-41.

Mudholkar, G. S., Srivastava, d. K. e Kollia, G. D. (1996). A generalization of the Weibull distribution with application to the analysis of survival data, Journal of the American Statistical Association 91: 1575-1583.

Nakamura, T. (1992). Proportional hazards model with covariates subject to measurement error, Biometrics 48: 829-839.

Natis, L. (2002). Modelos lineares hierárquicos, PhD thesis, Dissertação de mestrado. IME-USP.

Nelson, W. B. e Hahn, G. J. (1972). Linear estimation of a regression relationship from censored data. Part I-simple methods and their applications, Technometrics 14: $247-269$.

Oakes, D. (1989). Bivariate survival models induced by frailties, Journal of the American Statistical Association 84: 487-493.

O'Hagan, A. e Leonard, T. (1976). Bayes estimation subject to uncertainty about parameter constraints, Biometrika 63: 201-202.

Pettitt, A. N. (1986). Censored observations, repeated measures and mixed effects models: An approach using the EM algorithm and normal errors, Biometrika 73: 635-643.

Pinheiro, J. C. e Bates, D. M. (2000). Mixed-Effects Models in S and S-plus, New York: Springer-Verlag. 
Prentice, R. L. (1982). Covariate measurement errors and parameter estimation in a failure time regression model, Biometrika 69: 331-342.

Roberts, C. (1966). A correlation model useful in the study of twins, Journal of the American Statistical Society 61: 1184-1190.

Sahu, S. K. e Dey, D. K. (2000). A new class of multivariate skew distributions with aplications to bayesian regression models, Applied Statistics 49: 423-440.

Sahu, S. K., Dey, D. K. e Branco, M. D. (2003). A new class of multivariate skew distributions with aplications to Bayesian regression models, The Canadian Journal of Statistics 31: 129-150.

Sen, P. K. e Singer, J. M. (1993). Large Sample Methods in Statistics: An Introduction with Applications, New York: Chapman and Hall.

Shanno, D. F. (1970). Conditioning of quasi-newton methods for function minimization, Mathematics of Computation 24: 647-657.

Singer, J. M. e Andrade, J. M. (1997). Regression models for the analysis of pretest, posttest data, Biometrics 3: 729-735.

Valença, D. M. (2003). Teste de homogeneidade e estimação para dados de sobrevivência agrupados e com erros de medida, $\mathrm{PhD}$ thesis, Tese de doutorado. IMEUSP.

Vaupel, J. W., Manton, K. G. e Stallard, E. (1979). The impact of heterogeneity in individual frailty on the dynamics of mortality, Demography 16: 439-454.

Verbeke, G. e Molenberghs, G. (2000). Linear Mixed Models for Longitudinal Data, New York: Springer-Verlag. 
Vilca, F., Lachos, V. H. e Bolfarine, H. (2002). On testing statistics for comparing several measuring devices, RP, IMECC-UNICAMP 51/02.

Whittemore, A. e Altschuler, B. (1976). Lung cancer incidence in cigarette smokers:further analysis of doll and hill's data for british physicians, Biometrics 1: 8083.

Wolfinger, R., Tobias, R. e Sall, J. (1991). Mixed models: A future direction, SUGI 16: $1380-1388$.

Zhang, D. e Davidian, M. (2001). Linear mixed models with flexible distributions of random effects for longitudinal data, Biometrics 57: 795-802.

Zhao, Y. e Lee, A. (1998). Influence diagnostics for simultaneous equations models, Australian and New Zealand Journal Statistics 40: 345-357.

Zhu, H. T. e Lee, S. Y. (2001). Local influence for incomplete data models, Journal of the Royal Statistical Society B, 63: 111-126. 